\title{
Selective Phosphoranation of Unactivated Alkynes with Phosphonium Cation To Achieve Isoquinoline Synthesis
}

Hong Cui, ${ }^{\dagger a}$ Jinku Bai, ${ }^{\dagger a}$ Tianyu Ai, ${ }^{a}$ Ye Zhan, ${ }^{a}$ Guanzhong Li ${ }^{a}$ and Honghua Rao ${ }^{* a, b}$

${ }^{a}$ Department of Chemistry, Capital Normal University, Beijing 100048, P. R. China

${ }^{\mathrm{b}}$ Key Laboratory of Bioorganic Phosphorus Chemistry and Chemical Biology

(Ministry of Education), Department of Chemistry, Tsinghua University, Beijing 100084, P. R. China 


\section{Contents}

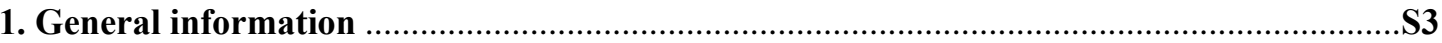

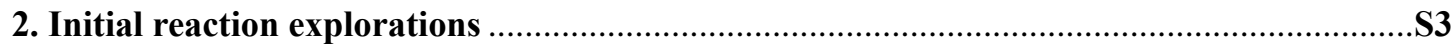

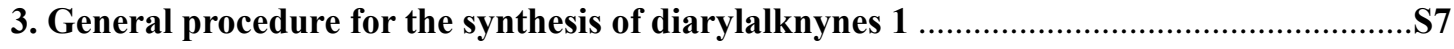

4. General procedure for the annulation of alkynes and nitriles ...........................................

5. The spectroscopic data for 3 -arylated isoquinoline derivatives $3 \ldots \ldots \ldots \ldots \ldots \ldots \ldots \ldots \ldots \ldots \ldots \ldots \ldots . . .514$

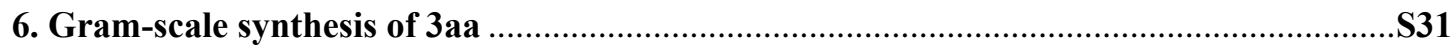

7. General procedure for the fluorination of 3aa

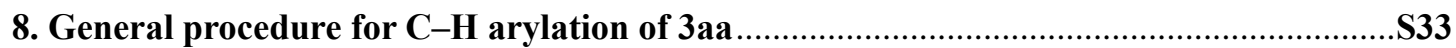

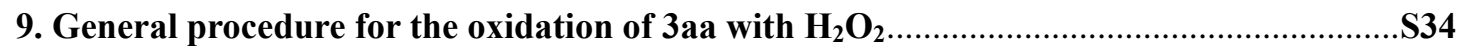

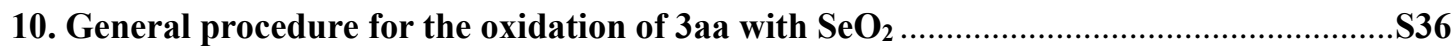

11. General procedure for $\mathrm{CDC}$ reaction of 3aa with phthalimide .......................................537

12. General procedure for the conversion of 3aa to nitrile ...................................................

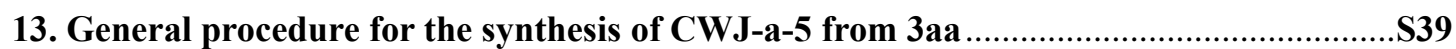

14. General procedure for the synthesis of pyrimidines ......................................................

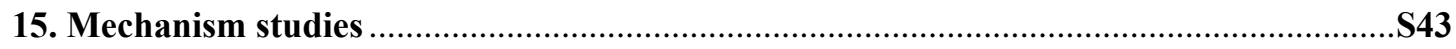

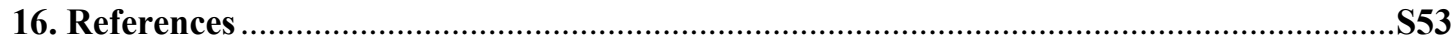

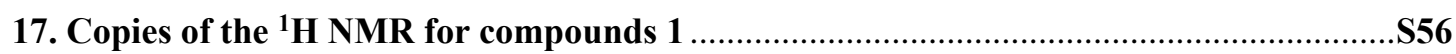

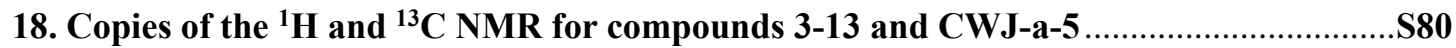

19. Copies of the spectrums for compounds 14 (analogue of intermediate VI) ……...........S171 


\section{General information}

All reactions were carried out under an argon atmosphere unless otherwise stated. All work-up and purification procedure were carried out with reagent-grade solvents.

Analytical thin-layer chromatography (TLC) and preparative thin layer chromatography (PTLC) were performed using silica gel 60 HF254 pre-coated plates (0.4-0.6 mm). The developed chromatography was analyzed by UV lamp (254 nm) as visualization methods. Column chromatography was performed on silica gel (300-400 mesh) with an appropriate solvent system (see details below).

Melting point was recorded on SGW X-4B.

Nuclear magnetic resonance (NMR) spectra were recorded on Varian $600 \mathrm{MHz}$ or $400 \mathrm{MHz}$ spectrometers.

Chemical shifts for ${ }^{1} \mathrm{H}-\mathrm{NMR}$ spectra are reported in parts per million (ppm) with the solvent resonance as the internal standard $\left(\mathrm{CDCl}_{3}: 7.26 \mathrm{ppm}\right)$. Chemical shifts for ${ }^{13} \mathrm{C}-\mathrm{NMR}$ spectra are reported in parts per million (ppm) with the solvent as the internal standard $\left(\mathrm{CDCl}_{3}: 77.0 \mathrm{ppm}\right)$. Data are reported as following: chemical shift, multiplicity ( $\mathrm{s}=$ singlet, $\mathrm{d}=$ doublet, $\mathrm{dd}=$ doublet of doublets, $\mathrm{t}=$ triplet, $\mathrm{td}=$ triplet of doublets, $\mathrm{q}$ $=$ quartet, $\mathrm{m}=$ multiplet, $\mathrm{br}=$ broad signal), coupling constant $(\mathrm{Hz})$, and integration .

High-resolution mass spectra were recorded on Thermo Scientific LTQ Orbitrap Discovery (Bremen, Germany).

Materials were purchased from Alfa-Aesar, Acros, Aldrich, Aladdin, EnergyChemical, Bide Pharmatech. Ltd., and Ouhe-Chemicals. Unless otherwise noted, commercial reagents were used without further purifications.

\section{Initial reaction explorations}




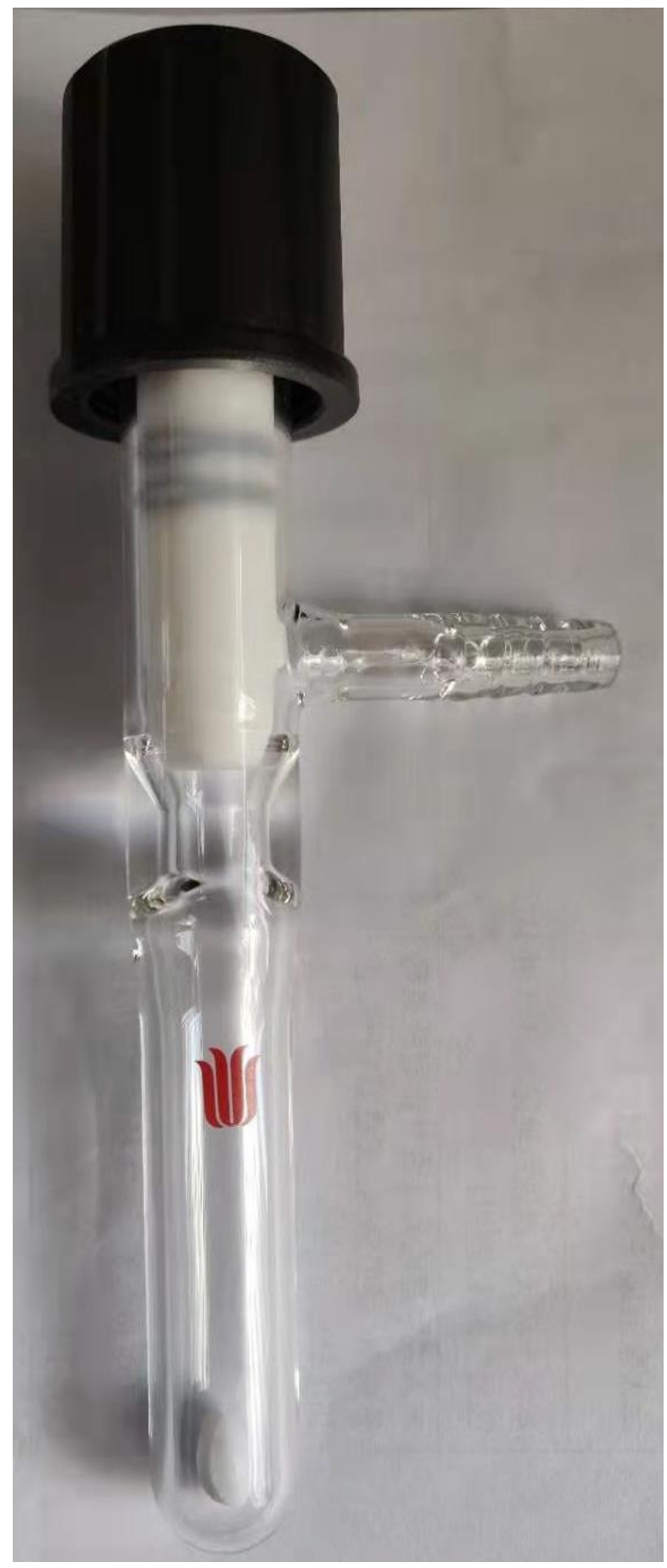

Scheme S1. 10-mL Schlenk tubes for our reaction (Taken by Honghua Rao)

As Miura/Hirano groups reported that treating $\mathrm{Ph}_{2} \mathrm{P}(\mathrm{O}) \mathrm{H}$ with $\mathrm{T}_{2} \mathrm{fO}$ can generate highly electrophilic phosphination reagent $\mathrm{Ph}_{2} \mathrm{P}-\mathrm{OTf}$, which can react with the alkynyl moiety to afford phosphirenium intermediate. ${ }^{[1]}$ We thus hypothesized that this highly electrophilic phosphination reagent $\mathrm{Ph}_{2} \mathrm{P}-\mathrm{OTf}$ might also reaction with alkynyl moiety, and the resulting phosphirenium intermediate might interact with a base equivalent (such as nitrogen containing species) intermolecularly (although this intermolecular 
process might be more difficult than intramolecular pathway). We thereby chose diphenylacetylene as the alkyne substrate:

Table S1. Initial reaction exploration by using diphenylacetylene as the substrate

\begin{tabular}{|c|c|c|c|c|c|c|c|c|}
\hline & $\begin{array}{l}\bar{\equiv} \\
1 \mathrm{a} h\end{array}$ & $\mathrm{CH}_{3} \mathrm{CN}$ & $\frac{[\mathrm{P}]}{\mathrm{CH}_{3} \mathrm{Cl}}$ & $\frac{\mathrm{Tf}_{2} \mathrm{O}, \mathrm{TfO}}{\mathrm{V}, 120^{\circ} \mathrm{C}, \mathrm{A}}$ & $\frac{\mathrm{f}, \text { Base }}{\text { rgon, } 24 \mathrm{~h}}$ & 3aa & & \\
\hline Entry & {$[\mathrm{P}]$} & $\begin{array}{c}\mathrm{Tf}_{2} \mathrm{O} \\
\text { (equiv) }\end{array}$ & $\begin{array}{c}\text { TfOH } \\
\text { (equiv) }\end{array}$ & $\begin{array}{c}\text { Base } \\
\text { (equiv) }\end{array}$ & $\begin{array}{l}\text { Solvent } \\
(\mathrm{mL})\end{array}$ & $\mathrm{T}\left({ }^{\circ} \mathrm{C}\right)$ & Time & Yield \\
\hline 1 & $\mathrm{Ph}_{2} \mathrm{P}(\mathrm{O}) \mathrm{H}$ & 1.0 & 0 & \begin{tabular}{|c|}
$2,6-d i \mathrm{Me}-$ \\
$\mathrm{Py}(1.0)$
\end{tabular} & $\begin{array}{c}\mathrm{CH}_{3} \mathrm{CN} \\
(2.0) \\
\end{array}$ & 120 & 24 & 9 \\
\hline 2 & $\begin{array}{l}(3,5 \text {-diMe- } \\
\mathrm{Ph})_{2} \mathrm{P}(\mathrm{O}) \mathrm{H}\end{array}$ & 1.0 & 0 & $\begin{array}{c}2,6-d i \mathrm{Me}- \\
\text { Py (1.0) }\end{array}$ & $\begin{array}{c}\mathrm{CH}_{3} \mathrm{CN} \\
(2.0)\end{array}$ & 120 & 24 & 23 \\
\hline 3 & $\begin{array}{c}(4-\mathrm{Me}- \\
\mathrm{Ph})_{2} \mathrm{P}(\mathrm{O}) \mathrm{H}\end{array}$ & 1.0 & 0 & $\begin{array}{c}2,6-d i \mathrm{Me}- \\
\mathrm{Py}(1.0)\end{array}$ & $\begin{array}{c}\mathrm{CH}_{3} \mathrm{CN} \\
(2.0) \\
\end{array}$ & 120 & 24 & 25 \\
\hline 4 & $\begin{array}{c}(2- \\
\text { Napht })_{2} \mathrm{P}(\mathrm{O}) \mathrm{H}\end{array}$ & 1.0 & 0 & $\begin{array}{c}2,6-d i \mathrm{Me}- \\
\text { Py }(1.0)\end{array}$ & $\begin{array}{c}\mathrm{CH}_{3} \mathrm{CN} \\
(2.0)\end{array}$ & 120 & 24 & 10 \\
\hline 5 & $\mathrm{Ph}_{2} \mathrm{P}(\mathrm{O}) \mathrm{H}$ & 1.0 & 0 & $\begin{array}{c}\text { 3-Me-Py } \\
(1.0)\end{array}$ & $\begin{array}{c}\mathrm{CH}_{3} \mathrm{CN} \\
(2.0) \\
\end{array}$ & 120 & 24 & 11 \\
\hline 6 & $\mathrm{Ph}_{2} \mathrm{P}(\mathrm{O}) \mathrm{H}$ & 1.0 & 0 & $\begin{array}{c}2-\mathrm{Ph}-\mathrm{Py} \\
(1.0)\end{array}$ & $\begin{array}{c}\mathrm{CH}_{3} \mathrm{CN} \\
(2.0)\end{array}$ & 120 & 24 & 15 \\
\hline 7 & $\mathrm{Ph}_{2} \mathrm{P}(\mathrm{O}) \mathrm{H}$ & 1.0 & 0 & $\begin{array}{c}\text { 4-OH-Py } \\
(1.0)\end{array}$ & $\begin{array}{c}\mathrm{CH}_{3} \mathrm{CN} \\
(2.0) \\
\end{array}$ & 120 & 24 & 16 \\
\hline 8 & $\mathrm{Ph}_{2} \mathrm{P}(\mathrm{O}) \mathrm{H}$ & 1.0 & 0 & $\begin{array}{l}\text { DBU } \\
(1.0)\end{array}$ & $\begin{array}{c}\mathrm{CH}_{3} \mathrm{CN} \\
(2.0)\end{array}$ & 120 & 24 & 12 \\
\hline 9 & $\mathrm{Ph}_{2} \mathrm{P}(\mathrm{O}) \mathrm{H}$ & 1.0 & 0 & $\mathrm{Et}_{3} \mathrm{~N}(1.0)$ & $\begin{array}{c}\mathrm{CH}_{3} \mathrm{CN} \\
(2.0) \\
\end{array}$ & 120 & 24 & 15 \\
\hline 10 & $\mathrm{Ph}_{2} \mathrm{P}(\mathrm{O}) \mathrm{H}$ & 1.0 & 0 & $\begin{array}{c}\text { DIPEA } \\
(1.0)\end{array}$ & $\begin{array}{c}\mathrm{CH}_{3} \mathrm{CN} \\
(2.0)\end{array}$ & 120 & 24 & 8 \\
\hline 11 & $\begin{array}{c}(2,3,6 \text {-triMe- } \\
\mathrm{Ph})_{2} \mathrm{P}(\mathrm{O}) \mathrm{H}\end{array}$ & 1.0 & 0 & \begin{tabular}{|c|}
$2,6-d i \mathrm{Me}-$ \\
$\mathrm{Py}(1.0)$
\end{tabular} & $\begin{array}{c}\mathrm{CH}_{3} \mathrm{CN} \\
(2.0) \\
\end{array}$ & 120 & 24 & 7 \\
\hline 12 & $\begin{array}{c}(2,3,6-t r i \mathrm{Me}- \\
\mathrm{Ph})_{2} \mathrm{P}(\mathrm{O}) \mathrm{H}\end{array}$ & 1.0 & 0 & \begin{tabular}{|r|}
$2,6-d i \mathrm{Bu}^{t}-$ \\
$\mathrm{Py}(1.0)$
\end{tabular} & $\begin{array}{c}\mathrm{CH}_{3} \mathrm{CN} \\
(2.0) \\
\end{array}$ & 120 & 24 & 12 \\
\hline 13 & $\mathrm{Ph}_{2} \mathrm{P}(\mathrm{O}) \mathrm{H}$ & 1.0 & 0 & $\begin{array}{c}2,6-d i \mathrm{Me}- \\
\mathrm{Py}(1.0)\end{array}$ & $\begin{array}{c}\mathrm{CH}_{3} \mathrm{CN} \\
(2.0) \\
\end{array}$ & 150 & 24 & 12 \\
\hline 14 & $\mathrm{Ph}_{2} \mathrm{P}(\mathrm{O}) \mathrm{H}$ & 1.0 & 0 & $\begin{array}{c}2,6-d i \mathrm{Me}- \\
\mathrm{Py}(1.0)\end{array}$ & $\begin{array}{c}\mathrm{CH}_{3} \mathrm{CN} \\
(1.5)\end{array}$ & 150 & 24 & 17 \\
\hline 15 & $\mathrm{Ph}_{2} \mathrm{P}(\mathrm{O}) \mathrm{H}$ & 1.0 & 0 & $\begin{array}{c}2,6-d i \mathrm{Me}- \\
\mathrm{Py}(1.0)\end{array}$ & $\begin{array}{c}\mathrm{CH}_{3} \mathrm{CN} \\
(0.5) \\
\end{array}$ & 150 & 24 & 21 \\
\hline 16 & $\mathrm{Ph}_{2} \mathrm{P}(\mathrm{O}) \mathrm{H}$ & 1.0 & 0 & \begin{tabular}{|c|}
$2,6-d i \mathrm{Me}-$ \\
$\mathrm{Py}(1.0)$
\end{tabular} & $\begin{array}{c}\mathrm{CH}_{3} \mathrm{CN} / \mathrm{DCE} \\
(0.5+0.25)\end{array}$ & 150 & 24 & 24 \\
\hline 17 & $\mathrm{Ph}_{2} \mathrm{P}(\mathrm{O}) \mathrm{H}$ & 1.0 & 0 & 2,6-diMe- & $\mathrm{CH}_{3} \mathrm{CN} / \mathrm{TCE}$ & 150 & 24 & 25 \\
\hline
\end{tabular}




\begin{tabular}{|l|c|c|c|c|c|c|c|c|}
\hline & & & & $\mathrm{Py}(1.0)$ & $(0.5+0.25)$ & & & \\
\hline 18 & $\mathrm{Ph}_{2} \mathrm{P}(\mathrm{O}) \mathrm{H}$ & $\mathbf{1 . 0}$ & $\mathbf{1 . 0}$ & $\begin{array}{c}2,6-d i \mathrm{Me}- \\
\mathrm{Py}(1.0)\end{array}$ & $\begin{array}{c}\mathrm{CH}_{3} \mathrm{CN} \\
(0.5)\end{array}$ & 150 & 24 & 33 \\
\hline
\end{tabular}

Reaction conditions: $\mathbf{1 a}(0.1 \mathrm{mmol} ; 1.0$ equiv), under argon atmosphere with other reagents, reaction temperature and reaction time indicated in the table. Yields are determined by ${ }^{1} \mathrm{H}$ NMR with mesitylene as the internal standard.

As the yields are not lower than $35 \%$, we wondered that a slightly electron-rich alkyne substrate might be helpful to higher yields. From the results in Table S1, we found that $\mathrm{Tf}_{2} \mathrm{O}$ and $\mathrm{TfOH}$ both as the additives might be helpful. Therefore, bis(ptolyl)acetylene was used to seek for more efficient conditions with $\mathrm{Tf}_{2} \mathrm{O}$ and $\mathrm{TfOH}$ as the additives:

Table S2. Initial reaction exploration by using bis( $p$-tolyl)acetylene as the substrate.

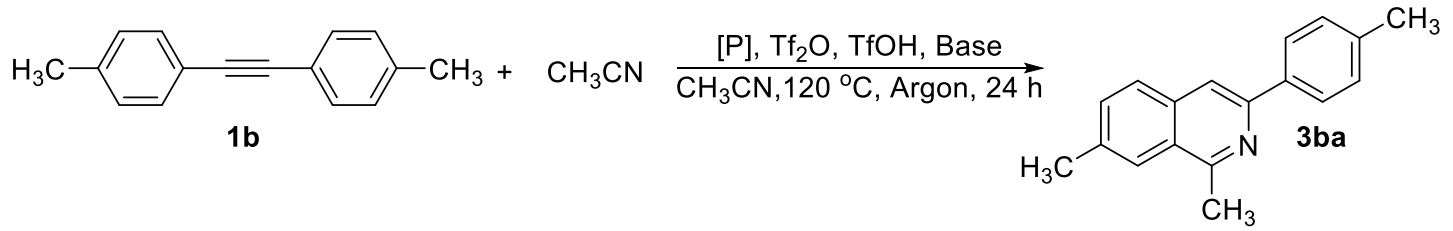

\begin{tabular}{|l|c|c|c|c|c|c|c|c|}
\hline Entry & {$[\mathrm{P}]$} & $\begin{array}{c}\mathrm{Tf}_{2} \mathrm{O} \\
\text { (equiv) }\end{array}$ & $\begin{array}{c}\mathrm{TfOH} \\
\text { (equiv) }\end{array}$ & Base (equiv) & $\begin{array}{c}\text { Solvent } \\
(\mathrm{mL})\end{array}$ & $\mathrm{T}\left({ }^{\circ} \mathrm{C}\right)$ & Time & Yield \\
\hline 1 & $\mathrm{Ph}_{2} \mathrm{P}(\mathrm{O}) \mathrm{H}$ & 1.0 & 1.0 & $\mathrm{DIPEA}(1.0)$ & $\begin{array}{c}\mathrm{CH}_{3} \mathrm{CN} \\
(0.5)\end{array}$ & 150 & 24 & 34 \\
\hline 2 & $\mathrm{Ph}_{2} \mathrm{P}(\mathrm{O}) \mathrm{H}$ & 1.0 & 1.0 & $\mathrm{PPh}_{3}(1.0)$ & $\begin{array}{c}\mathrm{CH}+\mathrm{CN} \\
(0.5)\end{array}$ & 150 & 24 & 49 \\
\hline 3 & $\mathrm{Ph}_{2} \mathrm{P}(\mathrm{O}) \mathrm{H}$ & 1.0 & 1.0 & $\mathrm{PPh}_{3}(1.0)$ & $\begin{array}{c}\mathrm{CH}_{3} \mathrm{CN} / \mathrm{DCE} \\
(0.4+0.1)\end{array}$ & 150 & 24 & 54 \\
\hline 4 & $\mathrm{Ph}_{2} \mathrm{P}(\mathrm{O}) \mathrm{H}$ & 1.0 & 1.0 & $\mathrm{PPh}_{3}(1.0)$ & $\begin{array}{c}\mathrm{CH}_{3} \mathrm{CN} / \mathrm{DCE} \\
(0.4+0.2)\end{array}$ & 150 & 24 & 59 \\
\hline 5 & - & 1.0 & 1.0 & $\mathrm{PPh}_{3}(1.0)$ & $\begin{array}{c}\mathrm{CH}_{3} \mathrm{CN} / \mathrm{DCE} \\
(0.4+0.2)\end{array}$ & 150 & 24 & 68 \\
\hline 6 & - & 1.2 & 1.0 & $\mathrm{PPh}_{3}$ & $\begin{array}{c}\mathrm{CH}_{3} \mathrm{CN} / \mathrm{DCE} \\
(0.4+0.2)\end{array}$ & 150 & 24 & 61 \\
\hline 7 & - & 0.6 & 1.0 & $\mathrm{PPh}_{3}(1.0)$ & $\begin{array}{c}\mathrm{CH}_{3} \mathrm{CN} / \mathrm{DCE} \\
(0.4+0.2)\end{array}$ & 150 & 24 & 69 \\
\hline 8 & - & 0.4 & 1.0 & $\mathrm{PPh}_{3}(1.0)$ & $\begin{array}{c}\mathrm{CH}_{3} \mathrm{CN} / \mathrm{DCE} \\
(0.4+0.2)\end{array}$ & 150 & 24 & 74 \\
\hline 9 & - & 1.0 & $\mathrm{PPh}_{3}(1.0)$ & $\begin{array}{c}\mathrm{CH}_{3} \mathrm{CN} / \mathrm{DCE} \\
(0.4+0.2)\end{array}$ & 150 & 24 & 70 \\
\hline
\end{tabular}




\begin{tabular}{|c|c|c|c|c|c|c|c|c|}
\hline 10 & - & 0.4 & 1.0 & \begin{tabular}{|c|}
$(4-\mathrm{Me}-\mathrm{Ph})_{3} \mathrm{P}$ \\
$(1.0)$
\end{tabular} & $\begin{array}{c}\mathrm{CH}_{3} \mathrm{CN} / \mathrm{DCE} \\
(0.4+0.2)\end{array}$ & 150 & 24 & 62 \\
\hline 11 & - & 0.4 & 1.0 & $\begin{array}{c}(3-\mathrm{Me}-\mathrm{Ph})_{3} \mathrm{P} \\
(1.0)\end{array}$ & $\begin{array}{c}\mathrm{CH}_{3} \mathrm{CN} / \mathrm{DCE} \\
(0.4+0.2)\end{array}$ & 150 & 24 & 60 \\
\hline 12 & - & 0.4 & 1.0 & \begin{tabular}{|c|}
$(4-\mathrm{OMe}-$ \\
$\mathrm{Ph})_{3} \mathrm{P}(1.0)$ \\
\end{tabular} & $\begin{array}{c}\mathrm{CH}_{3} \mathrm{CN} / \mathrm{DCE} \\
(0.4+0.2)\end{array}$ & 150 & 24 & 52 \\
\hline 13 & - & 0.4 & 1.0 & $\begin{array}{c}(4-\mathrm{F}-\mathrm{Ph})_{3} \mathrm{P} \\
(1.0)\end{array}$ & $\begin{array}{c}\mathrm{CH}_{3} \mathrm{CN} / \mathrm{DCE} \\
(0.4+0.2)\end{array}$ & 150 & 24 & 67 \\
\hline 14 & - & 0.4 & 1.0 & $\begin{array}{c}(4-\mathrm{Cl}-\mathrm{Ph})_{3} \mathrm{P} \\
(1.0)\end{array}$ & $\begin{array}{c}\mathrm{CH}_{3} \mathrm{CN} / \mathrm{DCE} \\
(0.4+0.2)\end{array}$ & 150 & 24 & 65 \\
\hline 15 & - & 0.4 & 1.0 & $\begin{array}{c}(2-\mathrm{Br}-\mathrm{Ph})_{3} \mathrm{P} \\
(1.0)\end{array}$ & $\begin{array}{c}\mathrm{CH}_{3} \mathrm{CN} / \mathrm{DCE} \\
(0.4+0.2)\end{array}$ & 150 & 24 & 52 \\
\hline 16 & - & 0.4 & 0.8 & $\mathrm{PPh}_{3}(1.0)$ & $\begin{array}{c}\mathrm{CH}_{3} \mathrm{CN} / \mathrm{DCE} \\
(0.4+0.2)\end{array}$ & 150 & 24 & 58 \\
\hline 17 & - & 0.4 & 1.2 & $\mathrm{PPh}_{3}(1.0)$ & $\begin{array}{c}\mathrm{CH}_{3} \mathrm{CN} / \mathrm{DCE} \\
(0.4+0.2)\end{array}$ & 150 & 24 & 73 \\
\hline 18 & - & 0.4 & 1.2 & $\mathrm{PPh}_{3}(1.2)$ & $\begin{array}{c}\mathrm{CH}_{3} \mathrm{CN} / \mathrm{DCE} \\
(0.4+0.2)\end{array}$ & 150 & 24 & 80 \\
\hline 19 & - & 0.4 & 1.4 & $\mathrm{PPh}_{3}(1.4)$ & $\begin{array}{c}\mathrm{CH}_{3} \mathrm{CN} / \mathrm{DCE} \\
(0.4+0.2)\end{array}$ & 150 & 24 & 84 \\
\hline 20 & - & 0.4 & 2.0 & $\mathrm{PPh}_{3}(2.0)$ & $\begin{array}{c}\mathrm{CH}_{3} \mathrm{CN} / \mathrm{DCE} \\
(0.4+0.2)\end{array}$ & 150 & 24 & 81 \\
\hline 21 & - & 0.4 & 1.4 & $\mathrm{PPh}_{3}(1.4)$ & $\begin{array}{c}\mathrm{CH}_{3} \mathrm{CN} / \mathrm{DCE} \\
(0.8+0.4)\end{array}$ & 150 & 6 & 89 \\
\hline 22 & - & 0.4 & 1.4 & $\mathrm{PPh}_{3}(1.4)$ & $\begin{array}{c}\mathrm{CH}_{3} \mathrm{CN} / \mathrm{DCE} \\
(0.8+0.4)\end{array}$ & 150 & 3 & 96 \\
\hline
\end{tabular}

Reaction conditions: $\mathbf{1 b}(0.1 \mathrm{mmol} ; 1.0$ equiv), under argon atmosphere with other reagents, reaction temperature and reaction time indicated in the table. Yields are determined by ${ }^{1} \mathrm{H}$ NMR with mesitylene as the internal standard

\section{General procedure for the synthesis of diarylalknynes $1^{[2]}$}

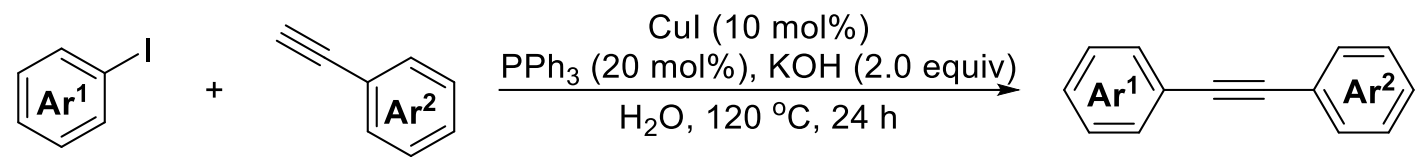

An oven-dried $10 \mathrm{~mL}$ Schlenk tube equipped with a stirring bar was charged with $\mathrm{CuI}(0.2 \mathrm{mmol}), \mathrm{PPh}_{3}(0.4 \mathrm{mmol})$ and $\mathrm{KOH}(99.9 \%$ purity, $4 \mathrm{mmol})$, evacuated three times and purged with argon. Then, $4 \mathrm{~mL}$ deionized water were added. After the suspension was stirred at room temperature for 10 minutes, ArI $(2 \mathrm{mmol})$ and arylacetylene $(2.6 \mathrm{mmol})$ were added. The reaction was shortly purged with argon and 
sealed. After $24 \mathrm{~h}$ at $120^{\circ} \mathrm{C}$ in oil bath, the mixture was cooled to temperature, extracted with EtOAc $(4 \times 20 \mathrm{~mL})$, the combined organic layer was dried over anhydrous $\mathrm{MgSO}_{4}$ and the solvent was removed under vacuum. The residue was purified by silica gel column (petroleum/EtOAc as the eluent) to give the analytically pure diarylalkynes $\mathbf{1}$.

\section{1,2-Di-p-tolylethyne (1b) ${ }^{[3]}$}

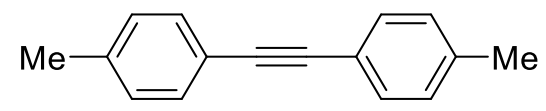

Isolated as an amorphous white solid in $82 \%$ yield (338 $\mathrm{mg}$ from $2 \mathrm{mmol}$ of 4iodotoluene).

${ }^{1}$ H NMR (600 MHz, CDCl $) \delta 7.42(\mathrm{~d}, J=8.10 \mathrm{~Hz}, 4 \mathrm{H}), 7.15(\mathrm{~d}, J=7.74 \mathrm{~Hz}, 4$ H), $2.37(6 \mathrm{H})$.

\section{1,2-Bis(4-tert-butylphenyl)ethyne (1c) ${ }^{[3]}$}

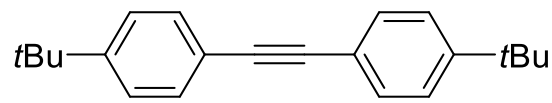

Isolated as an amorphous white solid in $72 \%$ yield (418 $\mathrm{mg}$ from $2 \mathrm{mmol}$ of 4tert-butyliodobenzene).

${ }^{1} \mathbf{H}$ NMR $\left(600 \mathrm{MHz}, \mathbf{C D C l}_{3}\right) \delta 7.47(\mathrm{~d}, J=8.40 \mathrm{~Hz}, 4 \mathrm{H}), 7.37(\mathrm{~d}, J=8.46 \mathrm{~Hz}, 4$ $\mathrm{H}), 1.33(18 \mathrm{H})$.

\section{1,2-Bis(4-fluorophenyl)ethyne (1d) ${ }^{[4]}$}

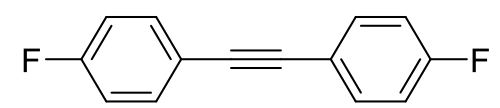

Isolated as a white solid in $65 \%$ yield $(279 \mathrm{mg}$ from $2 \mathrm{mmol}$ of 4fluoroiodobenzene).

${ }^{1}$ H NMR (600 MHz, CDCl $) \delta$ 7.52-7.48 (m, 4 H), 7.07-7.03 (m, 4 H).

\section{1,2-Di-o-tolylethyne (1e) $)^{[3]}$}

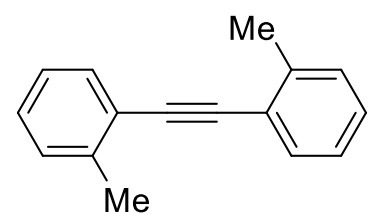

Isolated as an amorphous white solid in $61 \%$ yield $(252 \mathrm{mg}$ from $2 \mathrm{mmol}$ of 2iodotoluene).

${ }^{1}$ H NMR (600 MHz, CDCl $) \delta 7.53(\mathrm{~d}, J=8.40 \mathrm{~Hz}, 2 \mathrm{H}), 7.29-7.24(\mathrm{~m}, 4 \mathrm{H})$, 
7.21-7.18 (m, $2 \mathrm{H}), 2.55(6 \mathrm{H})$.

1,2-Bis(3,5-dimethylphenyl)ethyne (1f) ${ }^{[4]}$

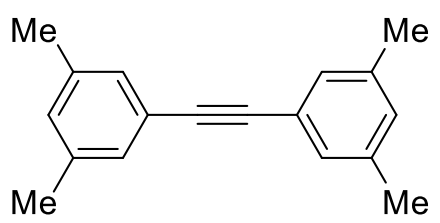

Isolated as an amorphous white solid in $75 \%$ yield $(352 \mathrm{mg}$ from $2 \mathrm{mmol}$ of 1 iodo-3,5-dimethylbenzene).

${ }^{1}$ H NMR (600 MHz, CDCl $) \delta 7.17$ (s, 4 H), 6.97 (s, $\left.2 \mathrm{H}\right), 2.32(12 \mathrm{H})$.

4-(Phenylethynyl)-1,1'-biphenyl (1g) ${ }^{[5]}$

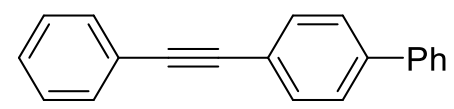

Isolated as an amorphous yellow solid in $81 \%$ yield (412 $\mathrm{mg}$ from $2 \mathrm{mmol}$ of $4-$ iodobiphenyl).

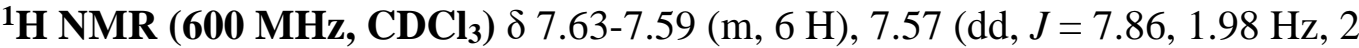
H), $7.47(\mathrm{t}, J=7.71 \mathrm{~Hz}, 2 \mathrm{H}), 7.39-7.35(\mathrm{~m}, 4 \mathrm{H})$.

1-Phenoxy-4-(phenylethynyl)benzene (1h) ${ }^{[6]}$

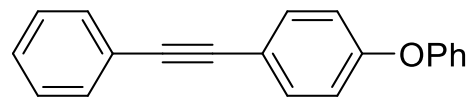

Isolated as an amorphous pale-yellow solid in 70\% yield (379 $\mathrm{mg}$ from $2 \mathrm{mmol}$ of 4-iododiphenyl ether).

${ }^{1}$ H NMR $(600$ MHz, CDCl $) \delta 7.53(\mathrm{dd}, J=7.56,1.50 \mathrm{~Hz}, 2 \mathrm{H}), 7.50(\mathrm{~d}, J=8.82$ $\mathrm{Hz}, 2 \mathrm{H}), 7.39-7.33$ (m, 5 H), $7.15(\mathrm{t}, J=7.41 \mathrm{~Hz}, 1 \mathrm{H}), 7.05(\mathrm{~d}, J=8.70 \mathrm{~Hz}, 2 \mathrm{H}), 6.98$ $(\mathrm{d}, J=8.82 \mathrm{~Hz}, 2 \mathrm{H})$.

\section{1-Methyl-4-(phenylethynyl)benzene (1i) ${ }^{[5]}$}

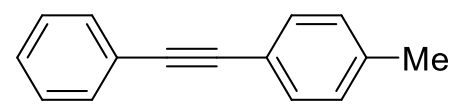

Isolated as an amorphous white powder in $74 \%$ yield $(285 \mathrm{mg}$ from $2 \mathrm{mmol}$ of 4 iodotoluene).

${ }^{1}$ H NMR $\left(600\right.$ MHz, $\left.\mathbf{C D C l}_{3}\right) \delta$ 7.54-7.52(m, $\left.2 \mathrm{H}\right), 7.44(\mathrm{~d}, J=8.04 \mathrm{~Hz}, 2 \mathrm{H})$, 7.36-7.32 (m, $3 \mathrm{H}), 7.16(\mathrm{~d}, J=7.86 \mathrm{~Hz}, 2 \mathrm{H}), 2.38(\mathrm{~s}, 3 \mathrm{H})$. 
1-(tert-Butyl)-4-(phenylethynyl)benzene $(1 \mathbf{j})^{[2]}$

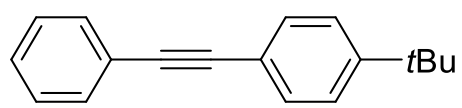

Isolated as an amorphous white solid in 69\% yield (324 mg from $2 \mathrm{mmol}$ of 4tert-butyliodobenzene).

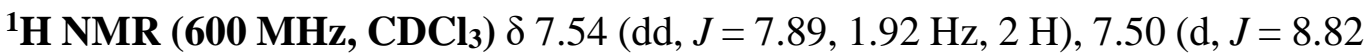
Hz, $2 \mathrm{H}), 7.39-7.32$ (m, $5 \mathrm{H}), 1.34$ (s, $9 \mathrm{H})$.

\section{1,3-Dimethyl-5-(phenylethynyl)benzene $(1 \mathrm{k})^{[7]}$}

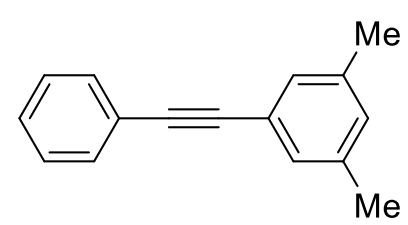

Isolated as an amorphous white powder in $67 \%$ yield (277 $\mathrm{mg}$ from $2 \mathrm{mmol}$ of 1iodo-3,5-dimethylbenzene).

${ }^{1}$ H NMR (600 MHz, CDCl $) \delta$ 7.55-7.52 (m, 2 H), 7.37-7.32 (m, 2 H), 7.18 (s, 2 H), 6.98 (s, $1 \mathrm{H})$.

\section{1-Fluoro-4-(phenylethynyl)benzene (11) ${ }^{[2]}$}

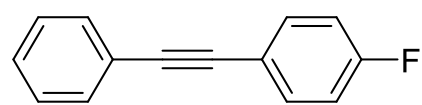

Isolated as an amorphous white powder in $67 \%$ yield (263 $\mathrm{mg}$ from $2 \mathrm{mmol}$ of 4fluoroiodobenzene).

${ }^{1}$ H NMR (600 MHz, CDCl $) \delta$ 7.53-7.50 (m, 4 H), 7.36-7.34 (m, 3 H), $7.05(\mathrm{t}, J$ $=8.67 \mathrm{~Hz}, 2 \mathrm{H})$.

1-Chloro-4-(phenylethynyl)benzene (1m) ${ }^{[7]}$

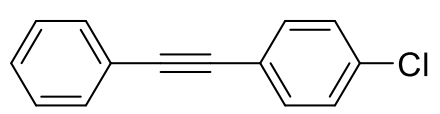

Isolated as an amorphous white powder in $78 \%$ yield $(332 \mathrm{mg}$ from $2 \mathrm{mmol}$ of 4 chloroiodobenzene).

${ }^{1}$ H NMR (600 MHz, CDCl $) \delta$ 7.53-7.52 (m, $\left.2 \mathrm{H}\right), 7.46(\mathrm{~d}, J=8.46 \mathrm{~Hz}, 2 \mathrm{H})$, 7.37-7.32 (m, $5 \mathrm{H})$.

\section{1-Bromo-4-(phenylethynyl)benzene $(1 \mathrm{n})^{[2]}$}




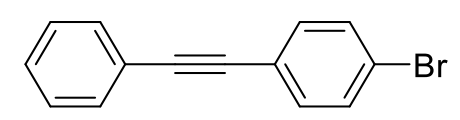

Isolated as an amorphous white solid in 79\% yield (406 $\mathrm{mg}$ from $2 \mathrm{mmol}$ of 4bromoiodobenzene).

${ }^{1}$ H NMR (600 MHz, CDCl $) \delta$ 7.53-7.52 (m, $\left.2 \mathrm{H}\right), 7.49(\mathrm{dt}, J=8.46,2.13 \mathrm{~Hz}, 2$ H), 7.39 (dt, $J=8.46,2.13 \mathrm{~Hz}, 2 \mathrm{H}), 7.37-7.34$ (m, $3 \mathrm{H})$.

\section{1-(Phenylethynyl)-4-(trifluoromethyl)benzene (10) ${ }^{[7]}$}

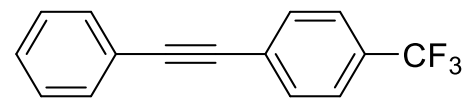

Isolated as an amorphous white solid in $67 \%$ yield $(331 \mathrm{mg}$ from $2 \mathrm{mmol}$ of 4iodobenzotrifluoride).

${ }^{1}$ H NMR (600 MHz, CDCl $) \delta 7.63(\mathrm{t}, J=8.46 \mathrm{~Hz}, 2 \mathrm{H}), 7.61(\mathrm{t}, J=8.64 \mathrm{~Hz}, 2$ H), 7.56-7.54 (m, $2 \mathrm{H})$, 7.38-7.37 (m, $3 \mathrm{H})$.

Methyl 4-(phenylethynyl)benzoate (1p) $)^{[5]}$

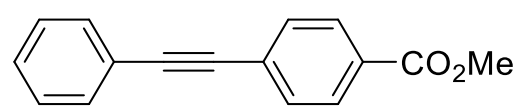

Isolated as an amorphous yellow solid in $65 \%$ yield $(309 \mathrm{mg}$ from $2 \mathrm{mmol}$ of methyl-4-iodobenzoate).

${ }^{1} \mathbf{H}$ NMR $\left(600 \mathrm{MHz}, \mathbf{C D C l}_{3}\right) \delta 8.02(\mathrm{~d}, J=8.40 \mathrm{~Hz}, 2 \mathrm{H}), 7.59(\mathrm{~d}, J=8.40 \mathrm{~Hz}, 2$ H), 7.56-7.54 (m, $2 \mathrm{H}), 7.37-7.36(\mathrm{~m}, 3 \mathrm{H}), 3.93$ (s, $3 \mathrm{H})$.

\section{4-(Phenylethynyl)benzonitrile (1q) ${ }^{[5]}$}

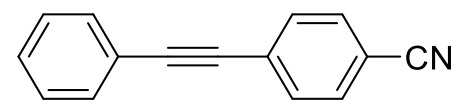

Isolated as an amorphous yellow solid in 67\% yield (274 $\mathrm{mg}$ from $2 \mathrm{mmol}$ of 4iodobenzonitrile).

${ }^{1}$ H NMR (600 MHz, CDCl $) \delta 7.64(\mathrm{~d}, J=8.58 \mathrm{~Hz}, 2 \mathrm{H}), 7.60(\mathrm{~d}, J=8.64 \mathrm{~Hz}, 2$ H), 7.56-7.54 (m, $2 \mathrm{H})$, 7.40-7.37 (m, $3 \mathrm{H})$.

\section{5-Bromo-1,3-dimethyl-2-(phenylethynyl)benzene (1r) ${ }^{[8]}$}

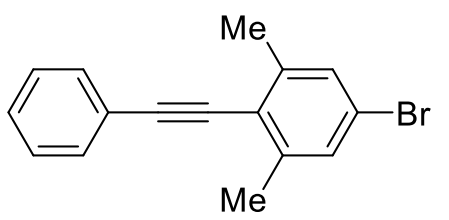


Isolated as an amorphous white solid in 59\% yield (338 $\mathrm{mg}$ from $2 \mathrm{mmol}$ of 5bromo-2-iodo-1,3-dimethylbenzene).

${ }^{1}$ H NMR (600 MHz, CDCl $) \delta$ 7.54-7.53 (m, 2 H), 7.38-7.35 (m, 3 H), 7.24 (s, 2 H), 2.48 (s, $6 \mathrm{H})$.

\section{5-Bromo-2-((4-ethylphenyl)ethynyl)-1,3-dimethylbenzene (1s)}

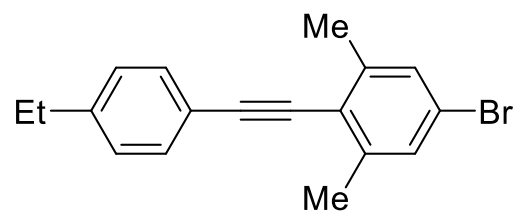

Isolated as an amorphous white solid in 52\% yield (328 $\mathrm{mg}$ from $2 \mathrm{mmol}$ of 5bromo-2-iodo-1,3-dimethylbenzene).

${ }^{1}$ H NMR (600 MHz, CDCl $) \delta 7.46(\mathrm{~d}, J=8.34 \mathrm{~Hz}, 2 \mathrm{H}), 7.23(\mathrm{~s}, 2 \mathrm{H}), 7.20(\mathrm{~d}$, $J=8.16 \mathrm{~Hz}, 2 \mathrm{H}), 2.68(\mathrm{q}, J=7.60 \mathrm{~Hz}, 2 \mathrm{H}), 2.48(\mathrm{~s}, 6 \mathrm{H}), 1.26(\mathrm{t}, J=7.65 \mathrm{~Hz}, 3 \mathrm{H})$.

${ }^{13}$ C NMR (150 MHz, CDCl 3$) \delta 144.8,142.0,131.4,129.6,128.0,122.3,121.5$, $120.6,99.0,85.6,28.8,20.8,15.4$.

\section{5-Bromo-2-((2-chlorophenyl)ethynyl)-1,3-dimethylbenzene (1t)}

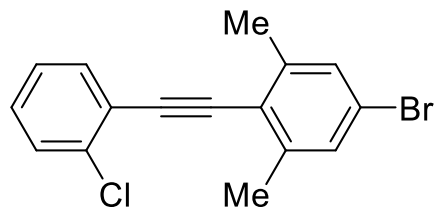

Isolated as an amorphous white solid in 53\% yield (339 $\mathrm{mg}$ from $2 \mathrm{mmol}$ of 5bromo-2-iodo-1,3-dimethylbenzene).

${ }^{1}$ H NMR (600 MHz, CDCl $)$ ) 7.57-7.56 (m, 1 H), 7.45-7.44 (m, 1 H), 7.29-7.24 (m, $4 \mathrm{H}), 2.52(\mathrm{~s}, 6 \mathrm{H})$.

${ }^{13}$ C NMR (150 MHz, CDCl 3$) \delta 142.4,135.6,133.1,129.7,129.4,129.3,126.5$, 123.4, 122.2, 121.8, 95.4, 91.4, 21.0.

\section{1-Methoxy-4-(p-tolylethynyl)benzene (1u) ${ }^{[5]}$}

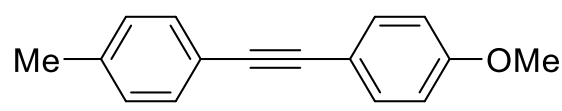

Isolated as an amorphous white solid in $60 \%$ yield $(267 \mathrm{mg}$ from $2 \mathrm{mmol}$ of 4 iodo-anisole).

${ }^{1}$ H NMR $\left(600 \mathrm{MHz}, \mathbf{C D C l}_{3}\right) \delta 7.46(\mathrm{~d}, J=8.76 \mathrm{~Hz}, 2 \mathrm{H}), 7.41(\mathrm{~d}, J=8.16 \mathrm{~Hz}, 2$ 
H), $7.15(\mathrm{~d}, J=7.86 \mathrm{~Hz}, 2 \mathrm{H}), 6.87(\mathrm{~d}, J=8.70 \mathrm{~Hz}, 2 \mathrm{H}), 3.83(\mathrm{~s}, 3 \mathrm{H}), 2.36(\mathrm{~s}, 3 \mathrm{H})$.

1-Chloro-4-(p-tolylethynyl)benzene (1v) ${ }^{[9]}$

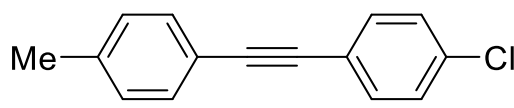

Isolated as an amorphous white solid in $69 \%$ yield $(314 \mathrm{mg}$ from $2 \mathrm{mmol}$ of $4-$ chloroiodobenzene).

${ }^{1}$ H NMR (600 MHz, CDCl 3$) \delta 7.45(\mathrm{dt}, J=8.52,2.22 \mathrm{~Hz}, 2 \mathrm{H}), 7.42(\mathrm{~d}, J=8.10$ Hz, $2 \mathrm{H}), 7.32$ (dt, $J=8.52,2.19 \mathrm{~Hz}, 2 \mathrm{H}), 7.16(\mathrm{~d}, J=7.74 \mathrm{~Hz}, 2 \mathrm{H}), 2.38$ (s, $3 \mathrm{H})$.

\section{2-(Phenylethynyl)thiophene (1w) $)^{[2]}$}

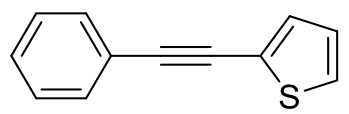

Isolated as an amorphous white solid in $32 \%$ yield (119 $\mathrm{mg}$ from $2 \mathrm{mmol}$ of 5bromo-2-iodothiophene).

${ }^{1} \mathbf{H}$ NMR (600 MHz, $\left.\mathbf{C D C l}_{3}\right) \delta$ 7.54-53 (m, $\left.3 \mathrm{H}\right)$, 7.39-7.33 (m, $\left.5 \mathrm{H}\right)$.

\section{General procedure for the annulation of alkynes and nitriles}

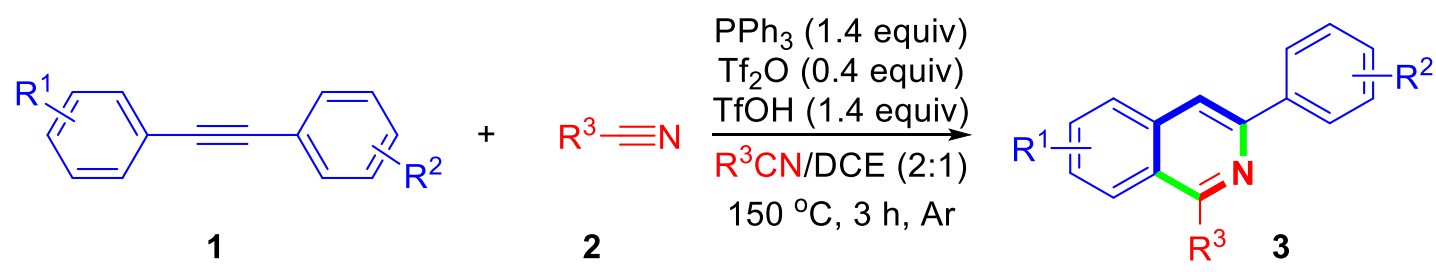

An oven-dried 10-mL Schlenk tube was charged with 1,2-bis(p-tolyl)ethyne 1 (0.1 mmol), triphenylphosphine (36.7 $\mathrm{mg}, 0.14 \mathrm{mmol})$. After the tube was filled with argon, 1,2-dichloroethane $(0.4 \mathrm{~mL})$ and $\mathbf{2}(0.8 \mathrm{~mL})$ were added by a syringe. Then the mixture was stirred at room temperature, and trifluoromethanesulfonic anhydride $(6 \mathrm{uL}, 0.04$ mmol) and trifluoromethanesulfonic acid (12.5 uL, $0.14 \mathrm{mmol})$ were added sequentially by a syringe under argon. The reaction mixture was sealed and heated at $150{ }^{\circ} \mathrm{C}$ in an oil bath for 3 hours. Upon completion, the resulting mixture was cooled to room temperature, and triethylamine $(0.5 \mathrm{ml})$ was added by a syringe. The resulting solution was stirred for more than 1 hour under air, filtered through a short silica gel 
pad and washed with ethyl acetate. The above solution was evaporated under vacuum, and the residue was purified by silica gel column with petroleum ether/acetone as the eluent to give the analytically pure product 3 .

\section{The spectroscopic data for 3-arylated isoquinoline derivatives 3}

\section{1-Methyl-3-phenylisoquinoline (3aa) ${ }^{[10]}$}<smiles>Cc1nc(-c2ccccc2)cc2ccccc12</smiles>

Synthesized according to the general procedure.

Obtained as a white solid, m.p. $38.3-40.9^{\circ} \mathrm{C}$.

Eluent: petroleum ether/acetone 120:1; isolated yield 91\% (19.8 mg from $17.9 \mathrm{mg}$ of 1a).

${ }^{1}$ H NMR (600 MHz, CDCl $) \delta 8.17-8.16(\mathrm{~m}, 2 \mathrm{H}), 8.12(\mathrm{~d}, J=8.34 \mathrm{~Hz}, 1 \mathrm{H})$, $7.93(\mathrm{~s}, 1 \mathrm{H}), 7.85(\mathrm{dd}, J=8.10,1.08 \mathrm{~Hz}, 1 \mathrm{H}), 7.67(\mathrm{t}, J=7.50 \mathrm{~Hz}, 1 \mathrm{H}), 7.57(\mathrm{td}, J=$ 7.91, $1.02 \mathrm{~Hz}, 1 \mathrm{H}), 7.52$ (td, $J=7.74,1.86 \mathrm{~Hz}, 2 \mathrm{H}), 7.44-7.41$ (m, $1 \mathrm{H}), 3.06$ (s, $3 \mathrm{H})$.

${ }^{13}$ C NMR (100 MHz, CDCl 3$) \delta$ 158.5, 149.9, 139.7, 136.7, 129.9, 128.6, 128.2, $127.5,126.9,126.7,126.5,125.5,115.1,22.6$.

FT-IR (KBr, $\left.\mathbf{c m}^{-1}\right) \tilde{v}_{\max } 3066,2953,2917,1617,1586,1563,1498,1439,1391$, 1364, 1332, 1032, 969, 925, 889, 855, 789, 770, 754, 700, 684, 663, 582, 559, 517.

HR-ESI-MS: $[\mathrm{M}+\mathrm{H}]^{+} \mathrm{m} / \mathrm{z}$ calcd for $\mathrm{C}_{16} \mathrm{H}_{14} \mathrm{~N}, 220.1121$; found 220.1120 .

\section{1,7-Dimethyl-3-p-tolylisoquinoline (3ba)}<smiles>Cc1ccc(-c2cc3ccc(C)cc3c(C)n2)cc1</smiles>

Synthesized according to the general procedure.

Obtained as a white solid, m.p. $87.1-88.9^{\circ} \mathrm{C}$.

Eluent: petroleum ether/acetone 150:1; isolated yield 92\% (22.7 mg from $20.6 \mathrm{mg}$ 
of $1 \mathbf{b})$.

${ }^{1}$ H NMR (600 MHz, CDCl $) \delta 8.04(\mathrm{dd}, J=8.19,1.86 \mathrm{~Hz}, 2 \mathrm{H}), 7.87(\mathrm{~s}, 1 \mathrm{H})$, $7.85(\mathrm{~s}, 1 \mathrm{H}), 7.74(\mathrm{~d}, J=8.34 \mathrm{~Hz}, 1 \mathrm{H}), 7.49(\mathrm{dd}, J=8.64,1.68 \mathrm{~Hz}, 1 \mathrm{H}), 7.31(\mathrm{~d}, J=$ $7.98 \mathrm{~Hz}, 2 \mathrm{H}), 3.02$ (s, $3 \mathrm{H}), 2.57$ (s, $3 \mathrm{H}), 2.43$ (s, $3 \mathrm{H})$.

${ }^{13}$ C NMR (100 MHz, CDCl 3$) \delta$ 157.6, 149.2, 137.9, 137.0, 136.4, 135.0, 132.1, $129.4,127.3,126.7,126.5,124.5,114.5,22.6,22.0,21.2$.

FT-IR (KBr, $\left.\mathbf{c m}^{-1}\right) \tilde{V}_{\max } 3066,2954,2914,1590,1566,1515,1439,1414,1398$, 1375, 1319, 1307, 1180, 1032, 1017, 969, 895, 872, 841, 826, 805, 720, 602, 518.

HR-ESI-MS: $[\mathrm{M}+\mathrm{H}]^{+} \mathrm{m} / \mathrm{z}$ calcd for $\mathrm{C}_{18} \mathrm{H}_{18} \mathrm{~N}, 248.1434$; found 248.1434 .

7-tert-Butyl-3-(4-tert-butylphenyl)-1-methylisoquinoline (3ca)

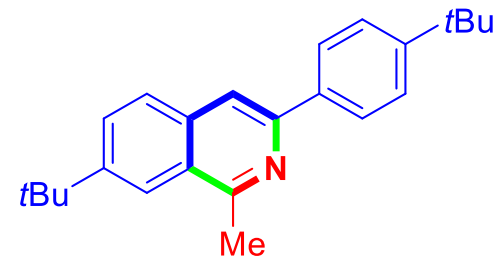

Synthesized according to the general procedure.

Obtained as a white solid, m.p. $116.8-119.4{ }^{\circ} \mathrm{C}$.

Eluent: petroleum ether/acetone 140:1; isolated yield 88\% (29.1 mg from $29.1 \mathrm{mg}$ of 1c)

${ }^{1}$ H NMR (600 MHz, CDCl $) \delta 8.08(\mathrm{~d}, J=8.64 \mathrm{~Hz}, 2 \mathrm{H}), 8.05(\mathrm{t}, J=0.93 \mathrm{~Hz}, 1$ H), $7.88(\mathrm{~s}, 1 \mathrm{H}), 7.81(\mathrm{~d}, J=8.58 \mathrm{~Hz}, 1 \mathrm{H}), 7.78(\mathrm{dd}, J=8.58,1.86 \mathrm{~Hz}, 1 \mathrm{H}), 7.54(\mathrm{~d}$, $J=8.64 \mathrm{~Hz}, 2 \mathrm{H}), 3.07$ (s, $3 \mathrm{H}), 1.47$ (s, $9 \mathrm{H}), 1.40(\mathrm{~s}, 3 \mathrm{H})$.

${ }^{13}$ C NMR (100 MHz, CDCl $) \delta$ 158.2, 151.2, 149.63, 149.60, 137.3, 135.0, 128.9, $127.3,126.7,126.4,125.7,120.4,114.5,35.2,34.7,31.4,31.3,22.7$.

FT-IR (KBr, $\left.\mathbf{c m}^{-1}\right) \tilde{v}_{\max } 3061,2962,2904,2869,1594,1562,1514,1460,1443$, $1413,1363,1318,1263,1194,1110,1015,968,899,889,875,843,828,630,544$.

HR-ESI-MS: $[\mathrm{M}+\mathrm{H}]^{+} \mathrm{m} / \mathrm{z}$ calcd for $\mathrm{C}_{24} \mathrm{H}_{30} \mathrm{~N}, 332.2373$; found 332.2365.

7-Fluoro-3-(4-fluorophenyl)-1-methylisoquinoline (3da) 


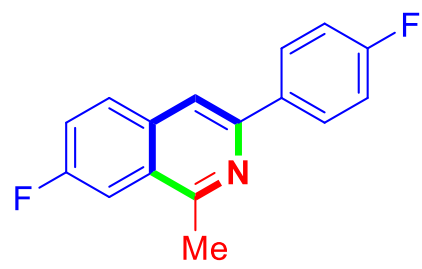

Synthesized according to the general procedure.

Obtained as a white solid, m.p. $125.4-127.1^{\circ} \mathrm{C}$.

Eluent: petroleum ether/acetone 200:1; isolated yield 40\% (10.1 mg from $21.5 \mathrm{mg}$ of $\mathbf{1 d})$.

${ }^{1}$ H NMR (600 MHz, CDCl $) \delta 8.10(\mathrm{dd}, J=8.28,5.88 \mathrm{~Hz}, 2 \mathrm{H}), 7.85(\mathrm{~s}, 1 \mathrm{H})$, $7.84(\mathrm{t}, J=7.23 \mathrm{~Hz}, 1 \mathrm{H}), 7.71(\mathrm{dd}, J=9.78,2.52 \mathrm{~Hz}, 1 \mathrm{H}), 7.45(\mathrm{td}, J=8.61,2.52 \mathrm{~Hz}$, $1 \mathrm{H}), 7.17(\mathrm{td}, J=8.61,0.72 \mathrm{~Hz}, 2 \mathrm{H}), 2.98(\mathrm{~s}, 3 \mathrm{H})$.

${ }^{13} \mathrm{C}$ NMR (100 MHz, CDCl $) \delta 163.2\left(\mathrm{C}-\mathrm{F},{ }^{1} J_{C-F}=246.1 \mathrm{~Hz}\right), 160.6\left(\mathrm{C}-\mathrm{F},{ }^{1} J_{C-F}=\right.$ $246.9 \mathrm{~Hz}), 158.0\left(\mathrm{C}-\mathrm{F},{ }^{4} J_{C-F}=5.7 \mathrm{~Hz}\right), 148.7\left(\mathrm{C}-\mathrm{F},{ }^{4} J_{C-F}=2.7 \mathrm{~Hz}\right), 135.7\left(\mathrm{C}-\mathrm{F},{ }^{4} J_{C-F}=\right.$ $3.3 \mathrm{~Hz}), 133.8,130.1\left(\mathrm{C}-\mathrm{F},{ }^{3} J_{C-F}=8.4 \mathrm{~Hz}\right), 128.6\left(\mathrm{C}-\mathrm{F},{ }^{3} J_{C-F}=8.2 \mathrm{~Hz}\right), 127.1\left(\mathrm{C}-\mathrm{F},{ }^{3} J_{C-}\right.$ $F=7.7 \mathrm{~Hz}), 120.6\left(\mathrm{C}-\mathrm{F},{ }^{2} J_{C-F}=25.1 \mathrm{~Hz}\right), 115.6\left(\mathrm{C}-\mathrm{F},{ }^{2} J_{C-F}=21.4 \mathrm{~Hz}\right), 114.5,109.3$ $\left(\mathrm{C}-\mathrm{F},{ }^{2} J_{C-F}=21.0 \mathrm{~Hz}\right), 22.7$.

FT-IR (KBr, cm$\left.^{-1}\right) \tilde{V}_{\max }$ 3062, 2953, 2926, 1602, 1580, 1515, 1498, 1441, 1423, $1394,1370,1318,1300,1276,1231,1189,1165,1125,1107,1015,930,904,870,839$, 795, 722, 607, 564, 537, 520, 508.

HR-ESI-MS: $[\mathrm{M}+\mathrm{H}]^{+} \mathrm{m} / \mathrm{z}$ calcd for $\mathrm{C}_{16} \mathrm{H}_{12} \mathrm{~F}_{2} \mathrm{~N}, 256.0932$; found 256.0930 .

\section{1,5-Dimethyl-3-o-tolylisoquinoline (3ea)}

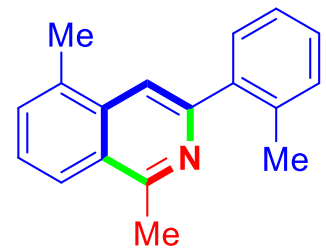

Synthesized according to the general procedure.

Obtained as a yellow oil.

Eluent: petroleum ether/acetone 120:1; isolated yield 84\% (20.8 mg from $20.6 \mathrm{mg}$ of $1 \mathbf{e})$.

${ }^{1}$ H NMR (600 MHz, CDCl $) \delta 8.04(\mathrm{dd}, J=8.22,1.08 \mathrm{~Hz}, 1 \mathrm{H}), 7.74(\mathrm{~s}, 1 \mathrm{H})$, 7.54-7.48 (m, 3 H), 7.33-7.30 (m, 3 H), 3.04 (s, $3 \mathrm{H}), 2.68$ (s, $3 \mathrm{H}), 2.43$ (s, $3 \mathrm{H})$. 
${ }^{13}$ C NMR (100 MHz, CDCl 3 ) $\delta$ 158.4, 152.4, 141.1, 136.2, 135.8, 134.3, 130.8, $130.4,130.1,128.0,126.4,126.0,125.9,123.7,115.4,22.8,20.5,19.1$.

FT-IR (KBr, cm$\left.^{-1}\right) \tilde{V}_{\max }$ 3063, 2952, 2922, 2856, 1726, 1612, 1590, 1564, 1495, $1481,1439,1382,1348,1272,1096,1034,972,905,882,872,852,804,772,755,728$, 691, 637, 580, 548.

HR-ESI-MS: $[\mathrm{M}+\mathrm{H}]^{+} \mathrm{m} / \mathrm{z}$ calcd for $\mathrm{C}_{18} \mathrm{H}_{18} \mathrm{~N}, 248.1434$; found 248.1430 .

\section{3-(3,5-Dimethylphenyl)-1,6,8-trimethylisoquinoline (3fa)}

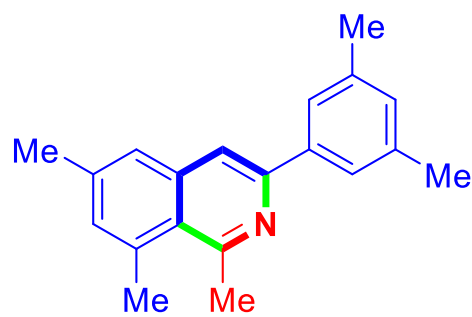

Synthesized according to the modified general procedure: using $\mathrm{CH}_{3} \mathrm{CN} / \mathrm{DCE}(1.2$ $\mathrm{mL}, 1: 2 \mathrm{v} / \mathrm{v}$ ratio) as the solvent.

Obtained as a white solid, m.p. $165.4-166.1^{\circ} \mathrm{C}$.

Eluent: petroleum ether/acetone 140:1; isolated yield 72\% (19.7 mg from $23.5 \mathrm{mg}$ of 1f).

${ }^{1}$ H NMR (600 MHz, CDCl $) \delta 7.78(\mathrm{~s}, 1 \mathrm{H}), 7.74(\mathrm{~s}, 2 \mathrm{H}), 7.45(\mathrm{~s}, 1 \mathrm{H}), 7.17$ (s, $1 \mathrm{H}), 7.04$ (s, $1 \mathrm{H}), 3.20$ (s, $3 \mathrm{H}), 2.91(\mathrm{~s}, 3 \mathrm{H}), 2.46$ (s, $3 \mathrm{H}), 2.43$ (s, $3 \mathrm{H})$.

${ }^{13}$ C NMR (100 MHz, CDCl $) \delta$ 158.1, 149.2, 139.4, 139.3, 138.1, 135.9, 132.5, $130.0,125.8,125.6,124.7,115.8,29.5,25.6,21.5,21.4$.

FT-IR (KBr, $\left.\mathbf{c m}^{-1}\right) \tilde{V}_{\max }$ 3026, 2970, 2911, 2853, 1621, 1605, 1589, 1565, 1482, 1468, 1457, 1435, 1379, 1358, 1329, 1218, 1192, 1035, 888, 861, 846, 789, 709, 603.

HR-ESI-MS: $[\mathrm{M}+\mathrm{H}]^{+} \mathrm{m} / \mathrm{z}$ calcd for $\mathrm{C}_{20} \mathrm{H}_{22} \mathrm{~N}, 276.1747$; found 276.1739 .

\section{3-((1,1'-Biphenyl)-4-yl)-1-methylisoquinoline (3ga) ${ }^{[10]}$}

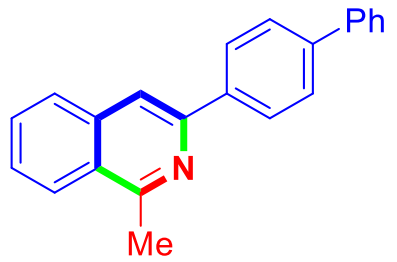

Synthesized according to the general procedure.

Obtained as a white solid, m.p. $144.3-145.3{ }^{\circ} \mathrm{C}$. 
Eluent: petroleum ether/acetone 120:1; isolated yield 72\% (21.2 mg from $25.5 \mathrm{mg}$ of 1g).

${ }^{1}$ H NMR (600 MHz, CDCl 3$) \delta 8.24(\mathrm{~d}, J=8.34 \mathrm{~Hz}, 2 \mathrm{H}), 8.14(\mathrm{~d}, J=8.40 \mathrm{~Hz}, 1$ H), $7.98(\mathrm{~s}, 1 \mathrm{H}), 7.87(\mathrm{~d}, J=8.16 \mathrm{~Hz}, 1 \mathrm{H}), 7.75(\mathrm{~d}, J=8.34 \mathrm{~Hz}, 2 \mathrm{H}), 7.70-7.67$ (m, $3 \mathrm{H}), 7.58(\mathrm{td}, J=7.59,1.26 \mathrm{~Hz}, 1 \mathrm{H}), 7.48(\mathrm{t}, J=7.74 \mathrm{~Hz}, 2 \mathrm{H}), 7.38(\mathrm{t}, J=7.41 \mathrm{~Hz}$, $1 \mathrm{H}), 3.07$ (s, $3 \mathrm{H})$.

${ }^{13}$ C NMR (100 MHz, CDCl 3$) \delta 158.7,149.5,141.1,140.9,138.7,136.8,130.1$, $128.8,127.7,127.5,127.41,127.39,127.1,126.9,126.7,125.7,115.2,22.7$.

FT-IR (KBr, cm$\left.^{-1}\right) \tilde{V}_{\max } 3051,2967,2922,1618,1597,1585,1567,1516,1486$, 1440, 1410, 1388, 1330, 1281, 1190, 1139, 1114, 1076, 1027, 1005, 993, 967, 948, 915, $903,882,859,837,782,768,749,732,699,683,585,542,515$.

HR-ESI-MS: $[\mathrm{M}+\mathrm{H}]^{+} \mathrm{m} / \mathrm{z}$ calcd for $\mathrm{C}_{22} \mathrm{H}_{18} \mathrm{~N}, 296.1434$; found 296.1428 .

\section{1-Methyl-3-(4-phenoxyphenyl)isoquinoline (3ha)}

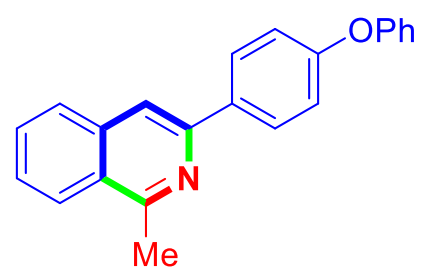

Synthesized according to the modified general procedure: using $\mathrm{CH}_{3} \mathrm{CN} / \mathrm{DCE}$ (1.2 $\mathrm{mL}, 1: 2 \mathrm{v} / \mathrm{v}$ ratio) as the solvent.

Obtained as a white solid, m.p. $77.6-82.8^{\circ} \mathrm{C}$.

Eluent: petroleum ether/acetone 140:1; isolated yield 64\% (19.8 mg from $27.1 \mathrm{mg}$ of 1h).

${ }^{1}$ H NMR (600 MHz, CDCl $)$ ) $\delta$ 8.13-8.12 (m, 3 H), $7.88(\mathrm{~s}, 1 \mathrm{H}), 7.85(\mathrm{~d}, J=8.64$ $\mathrm{Hz}, 1 \mathrm{H}), 7.67(\mathrm{td}, J=7.53,1.20 \mathrm{~Hz}, 1 \mathrm{H}), 7.56(\mathrm{td}, J=7.62,1.32 \mathrm{~Hz}, 1 \mathrm{H}), 7.37(\mathrm{t}, J$ $=8.01 \mathrm{~Hz}, 2 \mathrm{H}), 7.16-7.12(\mathrm{~m}, 3 \mathrm{H}), 7.08(\mathrm{dd}, J=8.07,1.26 \mathrm{~Hz}, 2 \mathrm{H}), 3.04(\mathrm{~s}, 3 \mathrm{H})$.

${ }^{13}$ C NMR (100 MHz, CDCl 3$) \delta$ 158.6, 157.6, 157.3, 149.4, 136.8, 135.0, 130.1, $129.8,128.5,127.6,126.7,126.4,125.7,123.3,119.2,118.9,114.8,22.7$.

FT-IR (KBr, $\left.\mathbf{c m}^{-1}\right) \tilde{v}_{\max } 3037,2916,1620,1607,1587,1566,1507,1487,1453$, $1438,1388,1332$, 1277, 1232, 1205, 1166, 1109, 1069, 1022, 913, 903, 885, 868, 834, $798,753,727,696,682,575,519$. 
HR-ESI-MS $[\mathrm{M}+\mathrm{H}]^{+} \mathrm{m} / \mathrm{z}$ calcd for $\mathrm{C}_{22} \mathrm{H}_{18} \mathrm{NO}, 312.1383$; found 312.1386.

1-Methyl-3-(p-tolyl)isoquinoline (3ia) ${ }^{[10]}$ and 1,7-Dimethyl-3-phenylisoquinoline $\left(3 i^{\prime}\right)^{[10]}(10: 1)$

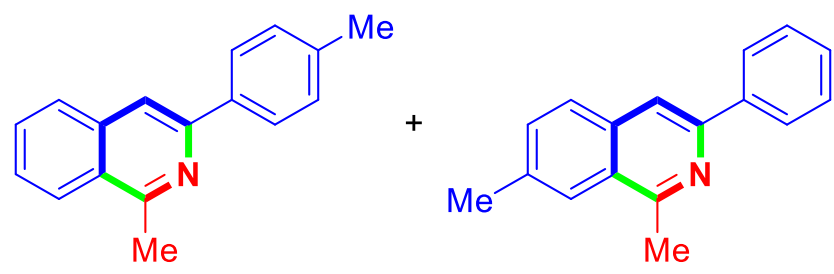

Synthesized according to the modified general procedure: using $\mathrm{CH}_{3} \mathrm{CN} / \mathrm{DCE}$ (1.2 $\mathrm{mL}, 1: 2 \mathrm{v} / \mathrm{v}$ ratio) as the solvent.

Obtained as a yellow oil.

Eluent: petroleum ether/acetone 140:1; isolated yield 83\% (19.3 mg from $19.3 \mathrm{mg}$ of 1i)

${ }^{1}$ H NMR (600 MHz, CDCl $) \delta 8.15(\mathrm{~d}, J=7.50 \mathrm{~Hz}, 0.22 \mathrm{H}$; minor), 8.11 (dd, $J$ $=8.31,1.14 \mathrm{~Hz}, 1 \mathrm{H}$; major), 8.07 (d, $J=7.80 \mathrm{~Hz}, 2 \mathrm{H}$; major), 7.90 (s, $1 \mathrm{H}$; major), 7.89 (s, $0.22 \mathrm{H}$; minor), 7.83 (d, $J=8.16 \mathrm{~Hz}, 1 \mathrm{H}$; major), 7.75 (d, $J=8.34 \mathrm{~Hz}, 0.14 \mathrm{H}$; minor), 7.65 (td, $J=7.53,1.20 \mathrm{~Hz}, 1 \mathrm{H}$; major), 7.56-7.53 (m, $1 \mathrm{H}$; major), 7.52-7.50 (m, 0.36 H; minor), 7.42-7.40 (m, 0.12 H; minor), 7.32 (d, J= 7.86 Hz, $2 \mathrm{H}$; major), 3.05 (s, $3 \mathrm{H}$; major), 3.03 (s, $0.34 \mathrm{H}$; minor), 2.58 (s, $0.30 \mathrm{H}$; minor), 2.44 (s, $3 \mathrm{H}$; major).

${ }^{13}$ C NMR (100 MHz, CDCl 3 ) $\delta 158.4$ (major), 157.7 (minor), 149.9 (major), 149.1 (minor), 139.8 (minor), 138.1 (major), 136.9 (major), 136.7 (major), 136.6 (minor), 132.1 (minor), 129.9 (major), 129.4 (major), 128.6 (minor), 128.1 (minor), 127.5 (major), 127.4 (minor), 126.82 (minor), 126.77 (major), 126.5 (major), 126.4 (major), 125.5 (major), 124.5 (minor), 115.0 (minor), 114.6 (major), 22.6 (major), 22.0 (minor), 21.2 (major).

FT-IR (KBr, $\left.\mathbf{c m}^{-1}\right) \tilde{v}_{\max }$ 3056, 2963, 2919, 2858, 1621, 1588, 1568, 1515, 1498, 1440, 1389, 1363, 1332, 1182, 1022, 969, 905, 880, 821, 749, 719, 682, 569, 518.

HR-ESI-MS: $[\mathrm{M}+\mathrm{H}]^{+} \mathrm{m} / \mathrm{z}$ calcd for $\mathrm{C}_{17} \mathrm{H}_{16} \mathrm{~N}$ 234.1277; found 234.1273. 

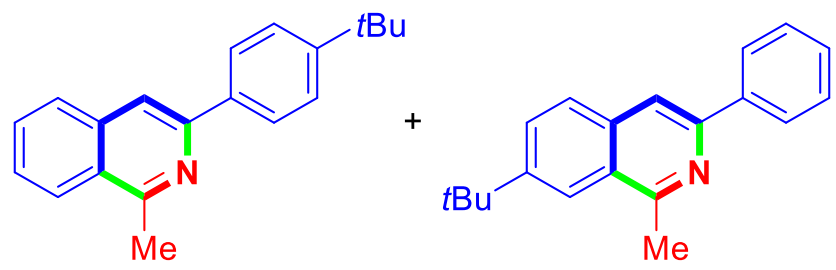

Synthesized according to the modified general procedure: using $\mathrm{CH}_{3} \mathrm{CN} / \mathrm{DCE}(1.2$ $\mathrm{mL}, 1: 2 \mathrm{v} / \mathrm{v}$ ratio) as the solvent.

Obtained as a yellow oil.

Eluent: petroleum ether/acetone 140:1; isolated yield 85\% (23.3 mg from $23.5 \mathrm{mg}$ of $\mathbf{1 j})$.

${ }^{1}$ H NMR (600 MHz, $\left.\mathbf{C D C l}_{3}\right) \delta 8.14(\mathrm{dd}, J=8.28,1.50 \mathrm{~Hz}, 0.22 \mathrm{H}$; minor), 8.12 (d, $J=7.98 \mathrm{~Hz}, 1 \mathrm{H}$; major), 8.07 (d, $J=8.40 \mathrm{~Hz}, 2 \mathrm{H}$; major), 8.05 (s, $0.11 \mathrm{H}$; minor), 7.90 (s, $1 \mathrm{H}$; major), 7.85 (d, $J=8.40 \mathrm{~Hz}, 1 \mathrm{H}$; major), 7.81 (d, $J=8.64 \mathrm{~Hz}, 0.10 \mathrm{H}$; minor), 7.78 (dd, $J=8.64,1.86 \mathrm{~Hz}, 0.10 \mathrm{H}$; minor), 7.66 (td, $J=7.22,1.14 \mathrm{~Hz}, 1 \mathrm{H}$; major), 7.56 (dd, $J=7.68,1.20 \mathrm{~Hz}, 1 \mathrm{H}$; major), 7.53 (dd, $J=7.47,1.86 \mathrm{~Hz}, 2 \mathrm{H}$; major), $7.50(\mathrm{t}, J=7.71 \mathrm{~Hz}, 0.21 \mathrm{H}$; minor), 7.40 (t, $J=7.32 \mathrm{~Hz}, 0.11 \mathrm{H}$; minor), 3.07 (s, 0.30 H; minor), 3.05 (s, $3 \mathrm{H}$; major), 1.47 (s, $0.9 \mathrm{H}$; minor), 1.39 (s, $9 \mathrm{H}$; major).

${ }^{13} \mathbf{C}$ NMR (100MHz, $\left.\mathbf{C D C l}_{3}\right) \delta 158.4$ (major), 158.3 (minor), 151.4 (major), 150.1 (major), 149.8 (minor), 149.4 (minor), 139.9 (minor), 137.0 (major), 136.8 (major), 134.9 (minor), 129.9 (major), 129.0 (minor), 128.7 (minor), 128.1 (minor), 127.5 (major), 127.3 (minor), 126.9 (minor), 126.7 (major), 126.5 (major), 126.4 (minor), 125.64 (major), 125.61 (major), 120.3 (minor), 114.9 (minor), 114.8 (major), 34.6 (minor), 31.3 (major), 31.2 (minor), 22.6 (major).

FT-IR (KBr, cm $\left.{ }^{-1}\right) \tilde{V}_{\max }$ 3056, 2961, 2915, 2866, 1622, 1591, 1568, 1515, 1441, $1389,1362,1333,1267,1112,1018,881,837,753,742,685,564,540$.

HR-ESI-MS: $[\mathrm{M}+\mathrm{H}]^{+} \mathrm{m} / \mathrm{z}$ calcd for $\mathrm{C}_{20} \mathrm{H}_{22} \mathrm{~N}, 276.1747$; found 276.1741 .

3-(3,5-Dimethylphenyl)-1-methylisoquinoline $(3 \mathrm{ka})^{[11]}$ and $1,6,8$-Trimethyl-3phenylisoquinoline (3ka') (3.4:1) 


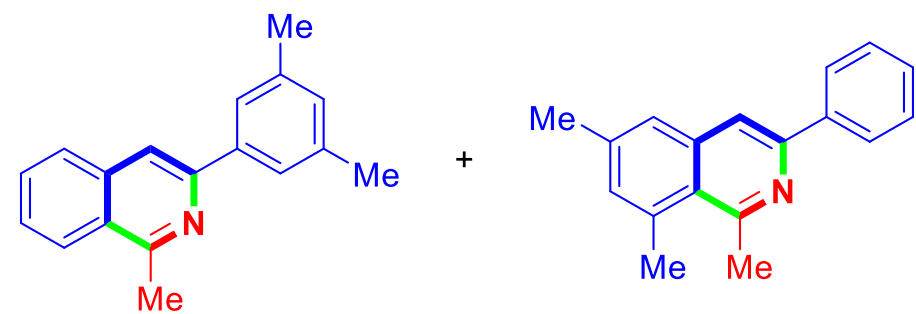

Synthesized according to the modified general procedure: using $\mathrm{CH}_{3} \mathrm{CN} / \mathrm{DCE}$ (1.2 $\mathrm{mL}, 1: 2 \mathrm{v} / \mathrm{v}$ ratio) as the solvent.

Obtained as a white solid, m.p. $80.0-80.9{ }^{\circ} \mathrm{C}$.

Eluent: petroleum ether/acetone 200:1; isolated yield $80 \%$ (19.7 mg from $20.7 \mathrm{mg}$ of $1 \mathbf{k})$.

${ }^{1}$ H NMR (600 MHz, CDCl $)$ ) $\delta$ 8.15-8.11 (m, 1.6 H), 7.91 (s, $1 \mathrm{H}$; major), 7.75 (d, $J=9.00 \mathrm{~Hz}, 1 \mathrm{H}$; major), 7.80 (s, $0.31 \mathrm{H}$; minor), 7.76 (s, $2 \mathrm{H}$; major), 7.66 (t, $J=7.47$ $\mathrm{Hz}, 1 \mathrm{H}$; major), 7.56 (t, $J=7.62 \mathrm{~Hz}, 1 \mathrm{H}$; major), 7.49 (t, $J=7.71 \mathrm{~Hz}, 0.60 \mathrm{H}$; minor), 7.45 (s, $0.30 \mathrm{H}$; minor), 7.40 (td, $J=7.35,1.08 \mathrm{~Hz}, 0.30 \mathrm{H}$; minor), 7.17 (s, $0.30 \mathrm{H}$; minor), 7.06 (s, $1 \mathrm{H}$; major), 3.19 (s, $0.88 \mathrm{H}$; minor), 3.05 (s, $3 \mathrm{H}$; major), 2.91 (s, 0.89 $\mathrm{H}$; minor), 2.46 (s, $0.92 \mathrm{H}$; minor), 2.44 (s, $6 \mathrm{H}$; major).

${ }^{13}$ C NMR (100MHz, $\mathbf{C D C l}_{3}$ ) $\delta 158.4$ (major), 158.2 (minor), 150.4 (major), 148.9 (minor), 139.8 (major), 139.6 (minor), 139.4 (minor), 139.3 (minor), 138.2 (major), 136.8 (major), 135.9 (minor), 132.6 (minor), 130.1 (major), 130.0 (major), 128.7 (major), 128.2 (minor), 127.6 (major), 126.8 (major), 126.64 (major), 126.56 (minor), 125.9 (minor), 125.7 (major), 124.9 (major), 115.7 (minor), 115.2 (major), 29.6 (minor), 25.6 (minor), 22.7 (major), 21.5 (major), 21.4 (minor).

FT-IR (KBr, $\left.\mathbf{c m}^{-1}\right) \tilde{v}_{\max }$ 3065, 2962, 2915, 2855, 1622, 1601, 1582, 1568, 1499, $1443,1391,1378,1363,1334,1191,1158,1031,872,854,837,785,753,710,694$, $681,601,591,528$.

HR-ESI-MS: $[\mathrm{M}+\mathrm{H}]^{+} \mathrm{m} / \mathrm{z}$ calcd for $\mathrm{C}_{18} \mathrm{H}_{18} \mathrm{~N}, 248.1434$; found 248.1431 .

\section{3-(4-Fluorophenyl)-1-methylisoquinoline $\quad(31 \mathrm{a})^{[10]}$ and 7-Fluoro-1-methyl-3- phenylisoquinoline $\left(3 \mathrm{la}^{\prime}\right)^{[10]}(4.7: 1)$}




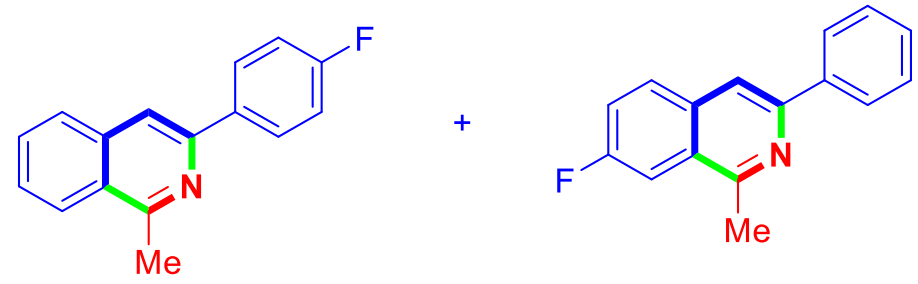

Synthesized according to the general procedure.

Obtained as a white solid, m.p. $67.2-68.0{ }^{\circ} \mathrm{C}$.

Eluent: petroleum ether/acetone 120:1; isolated yield $83 \%$ (19.6 mg from $19.6 \mathrm{mg}$ of 11)

${ }^{1}$ H NMR (600 MHz, CDCl $\left.{ }_{3}\right) \delta$ 8.14-8.11 (m, $\left.3.43 \mathrm{H}\right), 7.90$ (s, 0.21 H; minor), 7.85 (s, $1 \mathrm{H}$; major), 7.83 (d, $J=8.46 \mathrm{~Hz}, 1 \mathrm{H}$; major), 7.77-7.69 (m, $0.25 \mathrm{H}$; minor), 7.68-7.65 (m, $1 \mathrm{H}$; major), 7.58-7.55 (m, $1 \mathrm{H}$; major), 7.50 (t, $J=7.71 \mathrm{~Hz}, 0.46 \mathrm{H}$; minor), 7.46-7.40 (m, 0.48 H; minor), 7.20-7.16 (m, $2 \mathrm{H}$; major), 2.93 (s, 3H).

${ }^{13}$ C NMR (100 MHz, CDCl$) \delta 163.2\left(\mathrm{C}-\mathrm{F},{ }^{1} \mathrm{~J}_{\mathrm{C}-\mathrm{F}}=245.8 \mathrm{~Hz}\right.$; major), 160.6 (C-F, ${ }^{1} J_{C-F}=246.9 \mathrm{~Hz}$; minor), 158.7 (major), $157.9\left(\mathrm{C}-\mathrm{F},{ }^{4} J_{C-F}=5.5 \mathrm{~Hz}\right.$; minor), 149.7 (C$\mathrm{F},{ }^{4} J_{C-F}=2.8 \mathrm{~Hz}$; minor), 149.0 (major), 139.6 (minor), 136.8 (major), 136.0 (C-F, ${ }^{4} J_{C}$ ${ }_{F}=3.2 \mathrm{~Hz}$; major), 133.8 (minor), 130.2 (major), $130.1\left(\mathrm{C}-\mathrm{F},{ }^{3} J_{C-F}=8.4 \mathrm{~Hz}\right.$; minor), 128.8 (minor), 128.7 (C-F, ${ }^{3} J_{C-F}=8.2 \mathrm{~Hz}$; major), 128.4 (minor), 127.6 (major), 127.2 (C-F, ${ }^{3} J_{C-F}=7.7 \mathrm{~Hz}$; minor), 126.91 (minor), 126.87 (major), 126.5 (major), 125.7 (major), 120.5 (C-F, ${ }^{2} J_{C-F}=25.2 \mathrm{~Hz}$; minor), $115.6\left(\mathrm{C}-\mathrm{F},{ }^{2} J_{C-F}=21.3 \mathrm{~Hz}\right.$; major), 114.9 (major), $114.8\left(\mathrm{C}-\mathrm{F},{ }^{4} J_{C-F}=1.7 \mathrm{~Hz}\right.$; minor), $109.3\left(\mathrm{C}-\mathrm{F},{ }^{2} J_{C-F}=20.9 \mathrm{~Hz}\right.$; minor), 22.7 (minor), 22.6 (major).

FT-IR (KBr, $\left.\mathbf{c m}^{-1}\right) \tilde{v}_{\max }$ 3051, 2963, 2924, 1621, 1603, 1571, 1512, 1439, 1388, 1366, 1331, 1281, 1230, 1202, 1160, 1102, 1014, 970, 929, 905, 869, 836, 805, 774, $743,720,688,675,624,568,517$.

HR-ESI-MS: $[\mathrm{M}+\mathrm{H}]^{+} \mathrm{m} / \mathrm{z}$ calcd for $\mathrm{C}_{16} \mathrm{H}_{13} \mathrm{FN}, 238.1027$; found 238.1025.

\section{3-(4-Chlorophenyl)-1-methylisoquinoline $(3 \mathrm{ma})^{[10]}$ and 7-Chloro-1-methyl-3- phenylisoquinoline $\left(3 \mathrm{ma}^{\prime}\right)^{[12]}(1.7: 1)$}




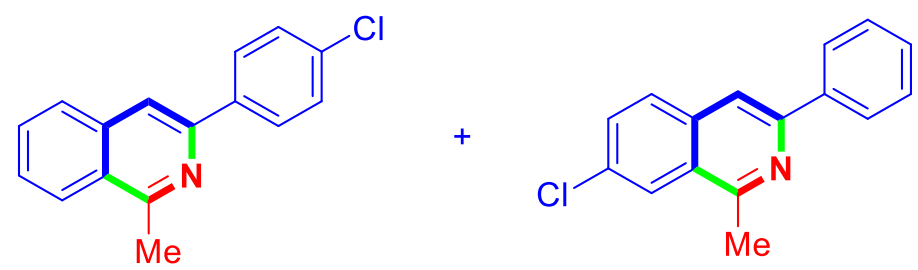

Synthesized according to the general procedure.

Obtained as a white solid, m.p. 93.0-96.2 ${ }^{\circ} \mathrm{C}$.

Eluent: petroleum ether/acetone 200:1; isolated yield $63 \%$ (15.9 mg from $21.3 \mathrm{mg}$ of $1 \mathbf{m})$.

${ }^{1}$ H NMR (600 MHz, CDCl $) \delta 8.13-8.07$ (m, 4.80 H), 7.87 (s, $1 \mathrm{H}$; major), 7.86 (s, $0.56 \mathrm{H}$; minor), 7.83 (d, $J=8.10 \mathrm{~Hz}$; major), 7.76 (d, $J=8.58 \mathrm{~Hz}, 0.60 \mathrm{H}$; minor), $7.67(\mathrm{td}, J=7.50,1.20 \mathrm{~Hz}, 1 \mathrm{H}$; major), 7.59-7.56 (m, $1.61 \mathrm{H}), 7.52-7.49(\mathrm{~m}, 1.22 \mathrm{H})$, 7.46 (dt, $J=8.64,2.34 \mathrm{~Hz}, 2 \mathrm{H}$; major), 7.42 (tt, $J=7.32,1.29 \mathrm{~Hz}, 0.61 \mathrm{H}$; minor), 3.02 (s, $3 \mathrm{H}$; major), 2.99 (s, $1.82 \mathrm{H}$; minor).

${ }^{13}$ C NMR (100 MHz, CDCl $\left._{3}\right) \delta 158.7$ (major), 157.7 (minor), 150.4 (minor), 148.7 (major), 139.4 (minor), 138.2 (major), 136.7 (major), 135.1 (minor), 134.4 (major), 132.2 (minor), 131.0 (minor), 130.2 (major), 129.3 (minor), 128.9 (major), 128.8 (minor), 128.6 (minor), 128.2 (major), 127.6 (major), 127.1 (minor), 127.03 (major), 126.96 (major), 126.7 (minor), 125.7 (major), 124.8 (minor), 115.1 (major), 114.7 (minor), 22.64 (minor), 22.62 (major).

FT-IR (KBr, $\left.\mathbf{c m}^{-1}\right) \tilde{V}_{\max } 3051,2956,2919,1610,1586,1565,1512,1485,1443$, $1413,1384,1362$, 1317, 1304, 1196, 1141, 1113, 1089, 1072, 969, 877, 865, 839, 825, $798,717,678,667,601,562,527,516$.

HR-ESI-MS: $[\mathrm{M}+\mathrm{H}]^{+} \mathrm{m} / \mathrm{z}$ calcd for $\mathrm{C}_{16} \mathrm{H}_{13} \mathrm{ClN}, 254.0730$; found 254.0731.

\section{3-(4-Bromophenyl)-1-methylisoquinoline $\quad(3 \mathrm{na})^{[10]}$ and 7-Bromo-1-methyl-3-} phenylisoquinoline (3na') ${ }^{[12]}(1.1: 1)$<smiles>Cc1nc(-c2ccc(Br)cc2)cc2ccc(Br)cc12</smiles>

Synthesized according to the general procedure.

Obtained as a yellow oil. 
Eluent: petroleum ether/acetone 200:1; isolated yield 59 \% (17.4 mg from $25.7 \mathrm{mg}$ of $1 n)$.

${ }^{1}$ H NMR (600 MHz, $\left.\mathbf{C D C l}_{3}\right) \delta 8.26(\mathrm{~d}, J=1.68 \mathrm{~Hz}, 0.88 \mathrm{H}$; minor), 8.13-8.11 (m, $2.81 \mathrm{H}), 8.02$ (d, $J=8.64 \mathrm{~Hz}, 2 \mathrm{H}$; major), 7.88 (s, $1 \mathrm{H}$; major), 7.86 (s, $0.92 \mathrm{H}$; minor), $7.84(\mathrm{~d}, J=8.16 \mathrm{~Hz}, 1 \mathrm{H}$; major), 7.73-7.70 (m, $1.85 \mathrm{H}), 7.67$ (t, $J=7.50 \mathrm{~Hz}, 1 \mathrm{H}$; major), 7.61 (d, $J=8.52 \mathrm{~Hz}, 1.85 \mathrm{H}$; minor), 7.58 (td, $J=7.64,1.26 \mathrm{~Hz}, 1 \mathrm{H}$; major), 7.50 (t, $J=7.71 \mathrm{~Hz}, 1.83 \mathrm{H}$; minor), 7.41 (t, $J=7.35 \mathrm{~Hz}, 0.88 \mathrm{H}$; minor), 3.03 (s, $3 \mathrm{H}$; major), 2.99 (s, $2.78 \mathrm{H}$; minor).

${ }^{13}$ C NMR (100 MHz, CDCl $_{3}$ ) $\delta 158.8$ (major), 157.7 (minor), 150.5 (minor), 148.7 (major), 139.4 (minor), 138.7 (major), 136.7 (major), 135.3 (minor), 133.5 (minor), 131.8 (major), 130.2 (major), 129.3 (minor), 128.8 (minor), 128.6 (minor), 128.5 (major), 128.1 (minor), 127.7 (major), 127.5 (minor), 127.1 (major), 127.0 (minor), 126.7 (major), 125.7 (major), 122.7 (major), 120.3 (minor), 115.2 (major), 114.8 (minor), 22.6.

FT-IR (KBr, cm$\left.^{-1}\right) \tilde{V}_{\max }$ 3061, 2962, 2919, 1620, 1588, 1565, 1497, 1439, 1409, 1387, 1364, 1331, 1070, 1026, 1008, 969, 903, 876, 830, 775, 749, 717, 692, 515.

HR-ESI-MS: [M+H] $]^{+} \mathrm{m} / \mathrm{z}$ calcd for $\mathrm{C}_{16} \mathrm{H}_{13} \mathrm{BrN}, 298.0226$; found 298.0226 .

1-Methyl-3-phenyl-7-(trifluoromethyl)isoquinoline (30a) and 1-Methyl-3-((4trifluoromethyl)phenyl)isoquinoline (3oa' $)^{[11]}(34: 1)$

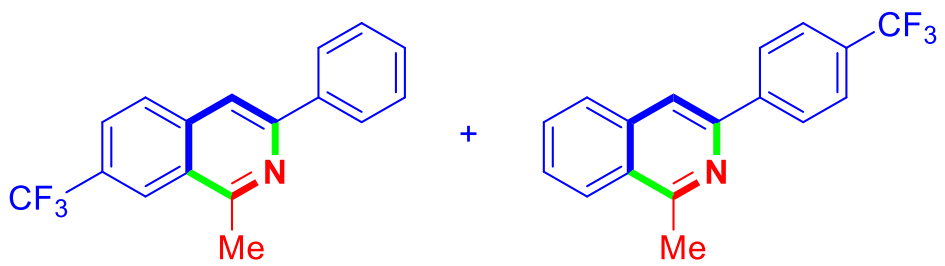

Synthesized according to the general procedure.

Obtained as a white solid, m.p. $68.6-69.1^{\circ} \mathrm{C}$.

Eluent: petroleum ether/acetone 140:1; isolated yield $23 \%(6.5 \mathrm{mg}$ from $24.6 \mathrm{mg}$ of 10).

${ }^{1}$ H NMR (600 MHz, CDCl $) \delta 8.40(\mathrm{~s}, 1 \mathrm{H}), 8.16(\mathrm{dd}, J=8.34,1.50 \mathrm{~Hz}, 2 \mathrm{H})$, 7.94-7.92 (m, $2 \mathrm{H}), 7.82(\mathrm{dd}, J=8.58,1.74 \mathrm{~Hz}, 1 \mathrm{H}), 7.52(\mathrm{t}, J=7.71 \mathrm{~Hz}, 2 \mathrm{H}), 7.44(\mathrm{t}$, $J=7.35 \mathrm{~Hz}, 1 \mathrm{H}), 3.07(\mathrm{~s}, 3 \mathrm{H})$. 
${ }^{13}$ C NMR (100 MHz, $\left.\mathbf{C D C l}_{3}\right) \delta$ 159.6, 152.0, 139.1, 138.2, 129.0, 128.90, 128.85, $128.80,128.4\left(\mathrm{C}-\mathrm{F},{ }^{2} J_{C-F}=32.3 \mathrm{~Hz}\right), 127.1,125.6\left(\mathrm{C}-\mathrm{F},{ }^{3} J_{C-F}=3.1 \mathrm{~Hz}\right), 125.4,124.0$ $\left(\mathrm{C}-\mathrm{F},{ }^{1} J_{C-F}=270.5 \mathrm{~Hz}\right), 123.5\left(\mathrm{C}-\mathrm{F},{ }^{3} J_{C-F}=4.5 \mathrm{~Hz}\right), 114.7,22.6$.

FT-IR (KBr, cm$\left.^{-1}\right) \tilde{V}_{\max }$ 3066, 2963, 2927, 1631, 1594, 1574, 1491, 1453, 1433, 1403, 1367, 1329, 1303, 1285, 1228, 1205, 1162, 1145, 1128, 1111, 1083, 1072, 1061, 1027, 967, 901, 886, 877, 843, 821, 779, 767, 732, 701, 690, 670, 639, 514.

HR-ESI-MS: $[\mathrm{M}+\mathrm{H}]^{+} \mathrm{m} / \mathrm{z}$ calcd for $\mathrm{C}_{17} \mathrm{H}_{13} \mathrm{~F}_{3} \mathrm{~N}, 288.0995$; found 288.0989.

\section{1-Methyl-3-phenylisoquinoline-7-carboxylate (3pa)}<smiles>CC(=O)c1ccc2cc(-c3ccccc3)nc(C)c2c1</smiles>

Synthesized according to the general procedure.

Obtained as a white solid, m.p. $153.0-154.0{ }^{\circ} \mathrm{C}$.

Eluent: petroleum ether/acetone 100:1; isolated yield $36 \%(9.9 \mathrm{mg}$ from $23.7 \mathrm{mg}$ of 1p).

${ }^{1}$ H NMR (600 MHz, $\left.\mathbf{C D C l}_{3}\right) \delta 8.89(\mathrm{~s}, 1 \mathrm{H}), 8.25(\mathrm{dd}, J=8.49,1.68 \mathrm{~Hz}, 1 \mathrm{H})$, $8.16(\mathrm{~d}, J=6.78 \mathrm{~Hz}, 2 \mathrm{H}), 7.95(\mathrm{~s}, 1 \mathrm{H}), 7.89(\mathrm{~d}, J=8.52 \mathrm{~Hz}, 1 \mathrm{H}), 7.51(\mathrm{t}, J=7.59 \mathrm{~Hz}$, $2 \mathrm{H}), 7.43(\mathrm{t}, J=7.32 \mathrm{~Hz}, 1 \mathrm{H}), 4.02(\mathrm{~s}, 3 \mathrm{H}), 3.12(\mathrm{~s}, 3 \mathrm{H})$.

${ }^{13}$ C NMR (100 MHz, CDCl $) \delta$ 166.6, 160.2, 152.0, 139.1, 129.5, 128.9, 128.8, $128.7,128.1,127.9,127.1,125.8,114.8,52.4,22.7$.

FT-IR (KBr, $\left.\mathbf{c m}^{-1}\right) \tilde{v}_{\max } 2959,2924,1710,1622,1566,1441,1421,1396,1326$, 1306, 1291, 1270, 1223, 1195, 1112, 1027, 970, 900, 885, 781, 768, 691, 678.

HR-ESI-MS: $[\mathrm{M}+\mathrm{H}]^{+} \mathrm{m} / \mathrm{z}$ calcd for $\mathrm{C}_{18} \mathrm{H}_{16} \mathrm{NO}_{2}, 278.1176$; found 278.1170 .

\section{1-Methyl-3-phenylisoquinoline-7-carbonitrile (3qa)}

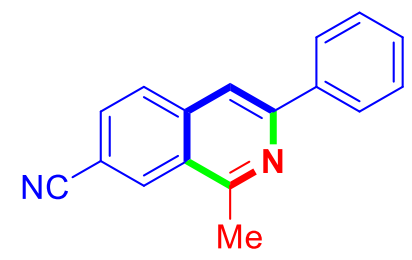

Synthesized according to the modified general procedure: using 2.8 equiv $\mathrm{PPh}_{3}$, 
0.8 equiv $\mathrm{Tf}_{2} \mathrm{O}$ and 2.8 equiv $\mathrm{TfOH}$.

Obtained as a yellow solid, m.p. $162.0-169.6^{\circ} \mathrm{C}$.

Eluent: petroleum ether/ethyl acetate 50:1; isolated yield $43 \%$ (10.4 mg from 20.3 mg of 1q).

${ }^{1}$ H NMR (600 MHz, CDCl $\left.\mathbf{l}_{3}\right) \delta 8.52(\mathrm{~s}, 1 \mathrm{H}), 8.16(\mathrm{~d}, J=7.02 \mathrm{~Hz}, 2 \mathrm{H}), 7.95(\mathrm{~s}$, $1 \mathrm{H}), 7.94(\mathrm{~d}, J=8.46 \mathrm{~Hz}, 1 \mathrm{H}), 7.79(\mathrm{dd}, J=8.40,1.56 \mathrm{~Hz}, 1 \mathrm{H}), 7.52(\mathrm{t}, J=7.68 \mathrm{~Hz}$, $2 \mathrm{H}), 7.45(\mathrm{t}, J=7.32 \mathrm{~Hz}, 1 \mathrm{H}), 3.08(\mathrm{~s}, 3 \mathrm{H})$.

${ }^{13}$ C NMR (100 MHz, $\left.\mathbf{C D C l}_{3}\right) \delta$ 159.3, 152.9, 138.7, 138.4, 132.2, 130.5, 129.3, $129.0,128.9,127.2,125.5,118.8,114.7,110.0,22.6$.

FT-IR (KBr, $\left.\mathbf{c m}^{-1}\right) \tilde{V}_{\max }$ 3079, 2955, 2921, 2219, 1794, 1618, 1590, 1559, 1499, 1437, 1412, 1389, 1321, 1309, 1226, 1203, 1178, 1145, 1070, 1027, 1000, 968, 914, $898,878,820,780,766,693,651,619,543,530$.

HR-ESI-MS: $[\mathrm{M}+\mathrm{H}]^{+} \mathrm{m} / \mathrm{z}$ calcd for $\mathrm{C}_{17} \mathrm{H}_{13} \mathrm{~N}_{2}, 245.1073$; found 245.1073 .

3-(4-Bromo-2,6-dimethylphenyl)-1-methylisoquinoline (3ra)

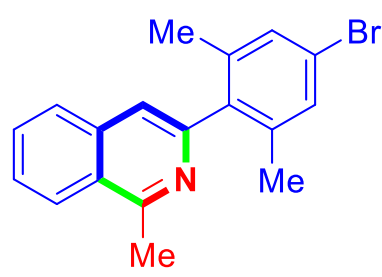

Synthesized according to the general procedure.

Obtained as a white solid, m.p. $115.0-116.5^{\circ} \mathrm{C}$.

Eluent: petroleum ether/acetone 200:1; isolated yield $73 \%$ (23.7 mg from $28.5 \mathrm{mg}$ of $1 \mathbf{r})$.

${ }^{1}$ H NMR (600 MHz, CDCl $) \delta 8.19(\mathrm{~d}, J=8.40 \mathrm{~Hz}, 1 \mathrm{H}), 7.82(\mathrm{~d}, J=8.16 \mathrm{~Hz}, 1$ H), $7.71(\mathrm{td}, J=7.56,1.20 \mathrm{~Hz}, 1 \mathrm{H}), 7.64(\mathrm{td}, J=7.70,1.32 \mathrm{~Hz}, 1 \mathrm{H}), 7.41(\mathrm{~s}, 1 \mathrm{H})$, $7.29(\mathrm{~s}, 2 \mathrm{H}), 3.02(\mathrm{~s}, 3 \mathrm{H}), 2.06(\mathrm{~s}, 6 \mathrm{H})$.

${ }^{13}$ C NMR (100 MHz, $\left.\mathbf{C D C l}_{3}\right) \delta 158.9,150.7,139.6,138.6,136.3,130.3,130.2$, $127.3,127.1,126.2,125.7,121.4,119.3,22.4,20.3$.

FT-IR (KBr, $\left.\mathbf{c m}^{-1}\right) \tilde{V}_{\max } 3046,2954,2920,2853,1619,1588,1571,1496,1447$, 1441, 1392, 1359, 1329, 1251, 1150, 1065, 1028, 969, 904, 891, 865, 858, 816, 790, 763, 723, 604, 584, 564, 531, 516. 
HR-ESI-MS: $[\mathrm{M}+\mathrm{H}]^{+} \mathrm{m} / \mathrm{z}$ calcd for $\mathrm{C}_{18} \mathrm{H}_{17} \mathrm{BrN}$, 326.0539; found 326.0543.

3-(4-Bromo-2,6-dimethylphenyl)-7-ethyl-1-methylisoquinoline (3sa)<smiles>CCc1ccc2cc(-c3c(C)cc(Br)cc3C)nc(C)c2c1</smiles>

Synthesized according to the general procedure.

Obtained as a white solid, m.p. $100.3-101.3{ }^{\circ} \mathrm{C}$.

Eluent: petroleum ether/acetone 140:1; isolated yield $77 \%(27.1 \mathrm{mg}$ from $31.3 \mathrm{mg}$ of $\mathbf{1 s})$.

${ }^{1}$ H NMR (600 MHz, CDCl 3$) \delta 7.95(\mathrm{~s}, 1 \mathrm{H}), 7.75(\mathrm{~d}, J=8.34 \mathrm{~Hz}, 1 \mathrm{H}), 7.59$ (d, $J=8.22, \mathrm{~Hz}, 1 \mathrm{H}), 7.37$ (s, $1 \mathrm{H}), 7.28$ (s, $2 \mathrm{H}), 3.00$ (s, $3 \mathrm{H}), 2.90$ (q, $J=7.60 \mathrm{~Hz}, 2 \mathrm{H}$ ), $2.06(\mathrm{~s}, 6 \mathrm{H}), 1.38$ (t, $J=7.62 \mathrm{~Hz}, 3 \mathrm{H})$.

${ }^{13}$ C NMR (100 MHz, CDCl$\left._{3}\right) \delta$ 158.2, 143.4, 138.7, 134.8, 131.4, 130.3, 127.3, $126.4,123.4,121.3,119.1,29.4,22.4,20.3,15.7$.

FT-IR (KBr, $\left.\mathbf{c m}^{-1}\right) \tilde{v}_{\max } 3055,2964,2926,2855,1627,1591,1574,1561,1497$, 1443, 1394, 1359, 1315, 1253, 1149, 1112, 1054, 1030, 966, 908, 894, 880, 851, 831, $816,561,541$.

HR-ESI-MS: $[\mathrm{M}+\mathrm{H}]^{+} \mathrm{m} / \mathrm{z}$ calcd for $\mathrm{C}_{20} \mathrm{H}_{21} \mathrm{BrN}, 354.0852$; found 354.0856.

3-(4-Bromo-2,6-dimethylphenyl)-5-chloro-1-methylisoquinoline (3ta)<smiles>Cc1cc(Br)cc(C)c1-c1cc2c(Cl)cccc2c(C)n1</smiles>

Synthesized according to the general procedure.

Obtained as a white solid, m.p. 151.6-152.3 ${ }^{\circ} \mathrm{C}$.

Eluent: petroleum ether/acetone 140:1; isolated yield $53 \%$ (18.8 mg from $32.0 \mathrm{mg}$ of 1t).

${ }^{1}$ H NMR (600 MHz, $\left.\mathbf{C D C l}_{3}\right) \delta 8.12(\mathrm{~d}, J=8.46 \mathrm{~Hz}, 1 \mathrm{H}), 7.82(\mathrm{~s}, 1 \mathrm{H}), 7.79(\mathrm{~d}$, $J=7.56 \mathrm{~Hz}, 1 \mathrm{H}), 7.55(\mathrm{t}, J=7.95 \mathrm{~Hz}, 1 \mathrm{H}), 7.31(\mathrm{~s}, 2 \mathrm{H}), 3.03(\mathrm{~s}, 3 \mathrm{H}), 2.07$ (s, $6 \mathrm{H})$. 
${ }^{13}$ C NMR (100 MHz, $\left.\mathbf{C D C l}_{3}\right) \delta$ 159.3, 151.9, 139.3, 138.5, 134.3, 131.8, 130.5, $130.3,127.1,126.9,124.7,121.7,115.8,22.7,20.3$.

FT-IR (KBr, cm$\left.^{-1}\right) \tilde{V}_{\max }$ 3051, 2951, 2917, 2852, 1615, 1581, 1557, 1482, 1443, $1418,1382,1346,1307,1252,1185,1162,1120,1087,1037,906,877,863,853,804$, $750,742,649,585$.

HR-ESI-MS: $[\mathrm{M}+\mathrm{H}]^{+} \mathrm{m} / \mathrm{z}$ calcd for $\mathrm{C}_{18} \mathrm{H}_{16} \mathrm{BrClN}, 360.0149$; found 360.0148 .

\section{3-(4-Methoxyphenyl)-1,7-dimethylisoquinoline (3ua)}

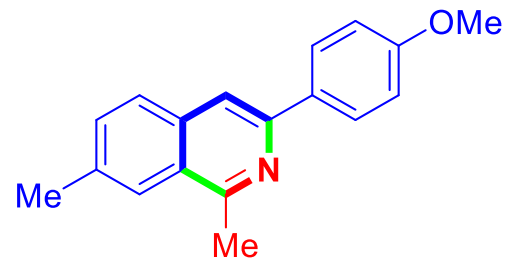

Synthesized according to the general procedure.

Obtained as a white solid, m.p. $88.8-90.2{ }^{\circ} \mathrm{C}$.

Eluent: petroleum ether/acetone 200:1; isolated yield $65 \%$ (17.0 mg from $22.3 \mathrm{mg}$ of $1 \mathbf{u})$.

${ }^{1}$ H NMR (600 MHz, CDCl $) \delta 8.08(\mathrm{dt}, J=8.82,2.43 \mathrm{~Hz}, 2 \mathrm{H}), 7.86(\mathrm{~s}, 1 \mathrm{H})$, $7.80(\mathrm{~s}, 1 \mathrm{H}), 7.72(\mathrm{~d}, J=8.28 \mathrm{~Hz}, 1 \mathrm{H}), 7.48(\mathrm{dd}, J=8.34,1.68 \mathrm{~Hz}, 1 \mathrm{H}), 7.02(\mathrm{dt}, J=$ 8.88, 2.52 Hz, $2 \mathrm{H}), 3.87$ (s, $3 \mathrm{H})$, ), 3.00 (s, $3 \mathrm{H}), 2.56$ (s, $3 \mathrm{H})$.

${ }^{13}$ C NMR (100 MHz, CDCl $\left.\mathbf{3}\right) \delta$ 159.9, 157.7, 149.0, 136.2, 135.1, 132.7, 132.1, $128.1,127.3,126.4,124.6,114.1,114.0,55.4,22.7,22.0$.

FT-IR (KBr, $\left.\mathbf{c m}^{-1}\right) \tilde{v}_{\max } 3062,2991,2967,2931,2835,1604,1590,1565,1514$, 1437, 1413, 1396, 1320, 1291, 1251, 1204, 1190, 1172, 1147, 1112, 1027, 967, 895, $870,846,835,815,802,778,732,681,638,602,565,522$.

HR-ESI-MS: $[\mathrm{M}+\mathrm{H}]^{+} \mathrm{m} / \mathrm{z}$ calcd for $\mathrm{C}_{18} \mathrm{H}_{18} \mathrm{NO}, 264.1383$; found 264.1380.

1-Chlorophenyl)-1,7-dimethylisoquinoline (3va) and 7-Chloro-1-methyl-3-(4methylphenyl)isoquinoline. (3va') (9:1)<smiles>Cc1ccc(-c2cc3ccc(Cl)cc3c(C)n2)cc1</smiles> 
Synthesized according to the modified general procedure: with $\mathrm{CH}_{3} \mathrm{CN} / \mathrm{DCE}$ (1.2 $\mathrm{mL}, 1: 2 \mathrm{v} / \mathrm{v}$ ratio).

Obtained as a yellow oil.

Eluent: petroleum ether/acetone 140:1; isolated yield $45 \%$ (11.9 mg from $22.7 \mathrm{mg}$ of $1 \mathbf{v})$.

${ }^{1}$ H NMR (600 MHz, CDCl $) \delta 8.07$ (d, $J=8.58 \mathrm{~Hz}, 0.24 \mathrm{H}$; minor), 8.04 (t, $J=$ $1.20 \mathrm{~Hz}, 1 \mathrm{H}$; major), 8.02 (d, $J=8.22 \mathrm{~Hz}, 2 \mathrm{H}$; major), 7.85 (s, $0.12 \mathrm{H}$; minor), 7.81 (s, $1 \mathrm{H}$; major), 7.72 (d, $J=8.70 \mathrm{~Hz}, 1 \mathrm{H}$; major), 7.70 (s, $0.06 \mathrm{H}$; minor), 7.55 (dd, $J=$ 8.67, $1.98 \mathrm{~Hz}, 1 \mathrm{H}$; major), 7.49 (dd, $J=8.34,1.68 \mathrm{~Hz}, 0.11 \mathrm{H}$; minor), 7.45 (d, $J=$ $8.52 \mathrm{~Hz}, 0.24 \mathrm{H}$; minor), 7.30 (d, J=7.74 Hz, $2 \mathrm{H}$; major), 2.99 (s, $0.36 \mathrm{H}$; minor), 2.97 (s, $3 \mathrm{H}$; major), 2.57 (s, $0.34 \mathrm{H}$; minor), 2.43 (s, $3 \mathrm{H}$; major).

${ }^{13}$ C NMR (100 MHz, $\mathbf{C D C l}_{3}$ ) $\delta 157.9$ (minor), 157.5 (major), 150.3 (major), 147.8 (minor), 138.4 (major), 138.2 (minor), 136.9 (minor), 136.5 (major), 135.0 (major), 134.8 (minor), 134.0 (minor), 132.3 (minor), 131.8 (major), 130.7 (major), 129.4 (major), 129.1 (major), 128.7 (minor), 128.0 (minor), 127.4 (minor), 126.8 (major), 126.7 (major), 124.6 (major), 124.5 (minor), 114.9 (minor), 114.1 (major), 22.5 (major), 22.0 (minor), 21.2 (major).

FT-IR (KBr, cm¹) $\tilde{V}_{\max }$ 3057, 2951, 2919, 1621, 1591, 1567, 1498, 1440, 1411, 1388, 1365, 1331, 1141, 1092, 1012, 969, 904, 878, 832, 778, 749, 720, 689, 681, 602, $542,517$.

HR-ESI-MS: $[\mathrm{M}+\mathrm{H}]^{+} \mathrm{m} / \mathrm{z}$ calcd for $\mathrm{C}_{17} \mathrm{H}_{15} \mathrm{ClN}, 268.0888$; found 268.0885.

\section{1-Methyl-3-(thiophen-2-yl)isoquinoline (3wa) ${ }^{[10]}$}

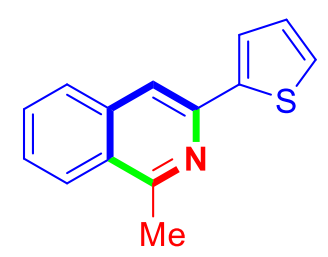

Synthesized according to the modified general procedure: with 1.0 equiv $\mathrm{TfOH}$. Obtained as a white solid, m.p. 37.6-38.2 ${ }^{\circ} \mathrm{C}$.

Eluent: petroleum ether/acetone 200:1; isolated yield $35 \%$ (7.8 mg from $18.5 \mathrm{mg}$ of $1 \mathbf{w})$. 
${ }^{1}$ H NMR (600 MHz, CDCl $) \delta 8.08(\mathrm{~d}, J=8.34 \mathrm{~Hz}, 1 \mathrm{H}), 7.83(\mathrm{~s}, 1 \mathrm{H}), 7.80(\mathrm{~d}$, $J=8.04 \mathrm{~Hz}, 1 \mathrm{H}), 7.69(\mathrm{dd}, J=3.66,1.20 \mathrm{~Hz}, 1 \mathrm{H}), 7.64(\mathrm{td}, J=7.50,1.14 \mathrm{~Hz}, 1 \mathrm{H})$, $7.53(\mathrm{tt}, J=7.62,1.38 \mathrm{~Hz}, 1 \mathrm{H}), 7.38(\mathrm{dd}, J=4.98,1.14 \mathrm{~Hz}, 1 \mathrm{H}), 7.14(\mathrm{dd}, J=4.98$, $3.72 \mathrm{~Hz}, 1 \mathrm{H}), 3.00$ (s, $3 \mathrm{H})$.

${ }^{13}$ C NMR (100 MHz, $\left.\mathbf{C D C l}_{3}\right) \delta$ 158.8, 145.3, 136.6, 130.2, 128.1, 127.4, 126.60, $126.57,125.8,123.8,113.1,22.4$.

FT-IR (KBr, cm$\left.^{-1}\right)$ Ṽmax 2960, 2920, 1614, 1584, 1865, 1491, 1447, 1440, 1382, 1365, 1327, 1261, 1236, 1193, 1157, 1137, 1083, 1058, 1027, 1014, 966, 957, 908, 895, $885,855,845,832,818,781,752,718,689,583,565,542,514$.

HR-ESI-MS: $[\mathrm{M}+\mathrm{H}]^{+} \mathrm{m} / \mathrm{z}$ calcd for $\mathrm{C}_{14} \mathrm{H}_{12} \mathrm{NS}, 226.0685$; found 226.0684 .

\section{7-Methyl-1-propyl-3-p-tolylisoquinoline (3bb)}

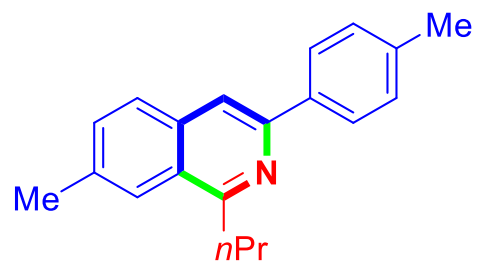

Synthesized according to the modified general procedure: with $n$-PrCN/DCE ( 0.6 $\mathrm{mL}, 1: 2 \mathrm{v} / \mathrm{v}$ ratio) as the solvent.

Obtained as a white solid, m.p. $94.5-96.4{ }^{\circ} \mathrm{C}$.

Eluent: petroleum ether/acetone 200:1; isolated yield $65 \%$ (17.8 mg from $20.6 \mathrm{mg}$ of $1 \mathbf{b})$.

${ }^{1}$ H NMR (600 MHz, CDCl $) \delta 8.06(\mathrm{~d}, J=8.10 \mathrm{~Hz}, 2 \mathrm{H}), 7.91(\mathrm{~s}, 1 \mathrm{H}), 7.85$ (s, $1 \mathrm{H}), 7.75(\mathrm{~d}, J=8.28 \mathrm{~Hz}, 1 \mathrm{H}), 7.47(\mathrm{~d}, J=8.34 \mathrm{~Hz}, 1 \mathrm{H}), 7.30(\mathrm{~d}, J=7.86 \mathrm{~Hz}, 2 \mathrm{H})$, $3.32(\mathrm{t}, J=7.74 \mathrm{~Hz}, 2 \mathrm{H}), 2.57(\mathrm{~s}, 3 \mathrm{H}), 2.42(\mathrm{~s}, 3 \mathrm{H}), 2.01$ (sextet, $J=7.52 \mathrm{~Hz}, 2 \mathrm{H})$, $1.13(\mathrm{t}, J=7.32 \mathrm{~Hz}, 3 \mathrm{H})$.

${ }^{13}$ C NMR (100 MHz, $\left.\mathbf{C D C l}_{3}\right) \delta 161.0,149.0,138.0,136.3,135.4,132.0,129.4$, $127.6,126.8,126.1,124.2,114.4,37.2,22.6,22.1,21.3,14.4$.

FT-IR (KBr, cm$\left.{ }^{-1}\right) \tilde{V}_{\max }$ 3052, 2960, 2925, 2867, 1591, 1566, 1513, 1458, 1427, $1421,1392,1374,1315,1259,1185,1178,1145,1109,1038,1016,897,875,839,818$, $812,779,741,722,597,540,520$.

HR-ESI-MS: $[\mathrm{M}+\mathrm{H}]^{+} \mathrm{m} / \mathrm{z}$ calcd for $\mathrm{C}_{20} \mathrm{H}_{22} \mathrm{~N}, 276.1747$; found 276.1745 . 


\section{1-iso-Butyl-7-methyl-3-p-tolylisoquinoline (3bc)}<smiles>Cc1ccc(-c2cc3ccc(C)cc3c(CC(C)C)n2)cc1</smiles>

Synthesized according to the general procedure.

Obtained as a yellow solid, m.p. $67.4-68.2{ }^{\circ} \mathrm{C}$.

Eluent: petroleum ether/acetone 200:1; isolated yield $32 \%$ (9.1 mg from $20.6 \mathrm{mg}$ of 1b).

${ }^{1}$ H NMR (600 MHz, CDCl $) \delta 8.05(\mathrm{~d}, J=8.16 \mathrm{~Hz}, 2 \mathrm{H}), 7.90(\mathrm{~s}, 1 \mathrm{H}), 7.85$ (s, $1 \mathrm{H}), 7.75(\mathrm{~d}, J=8.28 \mathrm{~Hz}, 1 \mathrm{H}), 7.48(\mathrm{dd}, J=8.34,1.62 \mathrm{~Hz}, 1 \mathrm{H}), 7.30(\mathrm{~d}, J=7.86 \mathrm{~Hz}$, $2 \mathrm{H}), 3.21$ (d, $J=7.08 \mathrm{~Hz}, 2 \mathrm{H}), 2.57$ (s, $3 \mathrm{H}), 2.46-2.43(\mathrm{~m}, 1 \mathrm{H}), 2.42(\mathrm{~s}, 3 \mathrm{H}), 1.06$ $(\mathrm{d}, J=6.60 \mathrm{~Hz}, 6 \mathrm{H})$.

${ }^{13}$ C NMR (100 MHz, CDCl 3$) \delta 160.4,148.9,137.9,136.2,135.4,131.9,129.4$, 127.6, 126.8, 126.6, 124.4, 114.2, 43.9, 28.8, 23.0, 22.1, 21.3.

FT-IR (KBr, cm$\left.^{-1}\right) \tilde{V}_{\max } 2955,2922,2863,1591,1561,1515,1478,1450,1421$, $1223,1216,1024,892,872,840,820,761,524$.

HR-ESI-MS: $[\mathrm{M}+\mathrm{H}]^{+} \mathrm{m} / \mathrm{z}$ calcd for $\mathrm{C}_{21} \mathrm{H}_{24} \mathrm{~N}, 290.1903$; found 290.1902 .

\section{Gram-scale synthesis of 3aa}

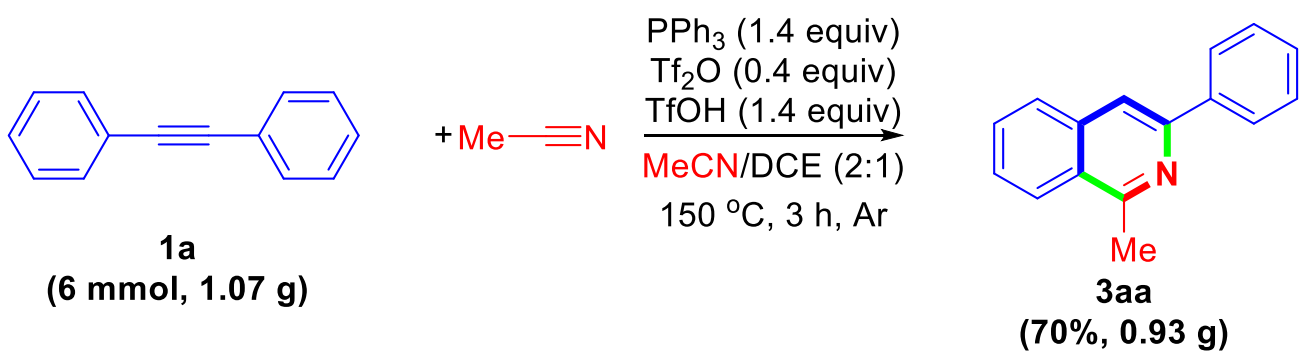

An oven-dried 250-mL Schlenk tube was charged with biphenylacetylene 1a (6.0 mmol; $1.07 \mathrm{~g})$, triphenylphosphine $(8.4 \mathrm{mmol} ; 2.203 \mathrm{~g})$. After the tube was filled with argon, 1,2-dichloroethane $(24 \mathrm{~mL})$ and $\mathrm{MeCN}(48 \mathrm{~mL})$ were added by a syringe. Then the mixture was stirred at room temperature, and trifluoromethanesulfonic anhydride $(0.36 \mathrm{~mL} ; 2.4 \mathrm{mmol})$ and trifluoromethanesulfonic acid $(0.75 \mathrm{~mL}, 8.4 \mathrm{mmol})$ were added sequentially by a syringe under argon. The reaction mixture was sealed and 
heated at $150{ }^{\circ} \mathrm{C}$ in an oil bath for 3 hours. Upon completion, the resulting mixture was cooled to room temperature, and triethylamine $(5 \mathrm{ml})$ was added by a syringe. The resulting solution was stirred for more than 1 hour under air, filtered through a short silica gel pad and washed with ethyl acetate. The above solution was evaporated under vacuum, and the residue was purified by silica gel column with petroleum ether/acetone 120:1 as the eluent to give the analytically pure product $3 \mathbf{a a}$ in $70 \%$ yield $(0.93 \mathrm{~g})$.

\section{General procedure for the fluorination of $3 \mathrm{aa}^{[13]}$}<smiles>[141I]c1nc(-c2ccccc2)cc2ccccc12</smiles>

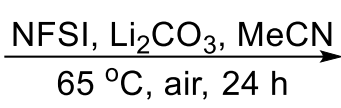

3aa<smiles>Cc1nc(-c2ccccc2)c(F)c2ccccc12</smiles>

4, $40 \%$

To a solution of 3aa $(0.1 \mathrm{mmol})$ in $\mathrm{CH}_{3} \mathrm{CN}(1.0 \mathrm{~mL})$ was added $\mathrm{N}$ fluorobenzenesulfonimide (NFSI; $0.3 \mathrm{mmol})$ and $\mathrm{Li}_{2} \mathrm{CO}_{3}(0.11 \mathrm{mmol})$ under air. The resulting reaction mixture sealed under air atmosphere, and heated in an oil-bath at $65^{\circ} \mathrm{C}$ for 24 hours. Then the reaction mixture was cooled to room temperature, diluted with $\mathrm{CH}_{2} \mathrm{Cl}_{2}(20 \mathrm{~mL})$ and washed with saturated aqueous solution of $\mathrm{NaHCO}_{3}(10 \mathrm{~mL} \times 3)$. The organic layer was dried over $\mathrm{MgSO}_{4}$, concentrated under vacuum. The residue was purified by column chromatography on silica gel to give analytically pure product 4 in $40 \%$ yield.

\section{4-Fluoro-1-methyl-3-phenylisoquinoline (4) ${ }^{[14]}$}

Obtained as a white solid, m.p. 39.7-40.3 ${ }^{\circ} \mathrm{C}$.

Eluent: Petroleum ether/ethyl acetate 20:1; isolated yield 40\% (9.5 mg from 21.9 mg of 3aa).

${ }^{1}$ H NMR (600 MHz, CDCl$) \delta 8.16(\mathrm{~d}, J=8.34 \mathrm{~Hz}, 1 \mathrm{H}), 8.12(\mathrm{~d}, J=8.28 \mathrm{~Hz}, 2$ H), $8.09(\mathrm{dd}, J=6.99,1.50 \mathrm{~Hz}, 1 \mathrm{H}), 7.76(\mathrm{t}, J=7.38 \mathrm{~Hz}, 1 \mathrm{H}), 7.64(\mathrm{t}, J=7.35 \mathrm{~Hz}, 1$ H), $7.53(\mathrm{t}, J=7.77 \mathrm{~Hz}, 2 \mathrm{H}), 7.43(\mathrm{t}, J=7.41 \mathrm{~Hz}, 1 \mathrm{H}), 2.99(\mathrm{~s}, 3 \mathrm{H})$.

${ }^{13}$ C NMR (100 MHz, CDCl 3$) \delta 153.9\left(\mathrm{C}-\mathrm{F},{ }^{4} J_{C-F}=5.7 \mathrm{~Hz}\right), 151.4\left(\mathrm{C}-\mathrm{F},{ }^{1} J_{C-F}=\right.$ $259.8 \mathrm{~Hz}), 135.6\left(\mathrm{C}-\mathrm{F},{ }^{3} J_{C-F}=6.8 \mathrm{~Hz}\right), 134.7\left(\mathrm{C}-\mathrm{F},{ }^{2} J_{C-F}=10.4 \mathrm{~Hz}\right), 130.3\left(\mathrm{C}-\mathrm{F},{ }^{4} J_{C-F}\right.$ 
$=3.4 \mathrm{~Hz}), 129.0\left(\mathrm{C}-\mathrm{F},{ }^{2} J_{C-F}=5.9 \mathrm{~Hz}\right), 128.5,128.4,128.0\left(\mathrm{C}-\mathrm{F},{ }^{4} J_{C-F}=2.4 \mathrm{~Hz}\right), 127.7$, $127.6\left(\mathrm{C}-\mathrm{F},{ }^{3} J_{C-F}=2.7 \mathrm{~Hz}\right), 125.6,120.5\left(\mathrm{C}-\mathrm{F},{ }^{3} J_{C-F}=6.0 \mathrm{~Hz}\right), 22.0$.

FT-IR (KBr, $\left.\mathbf{c m}^{-1}\right) \tilde{V}_{\max }$ 3055, 2960, 2923, 2855, 1958, 1622, 1589, 1573, 1503, 1443, 1339, 1309, 1261, 1182, 1153, 1076, 1054, 918, 866, 803, 740, 695, 616, 548.

HR-ESI-MS: $[\mathrm{M}+\mathrm{H}]^{+} \mathrm{m} / \mathrm{z}$ calcd for $\mathrm{C}_{16} \mathrm{H}_{13} \mathrm{FN}, 238.1027$; found 238.1026.

\section{General procedure for $\mathbf{C}-\mathbf{H}$ arylation of $\mathbf{3} \mathbf{a a}^{[15]}$}

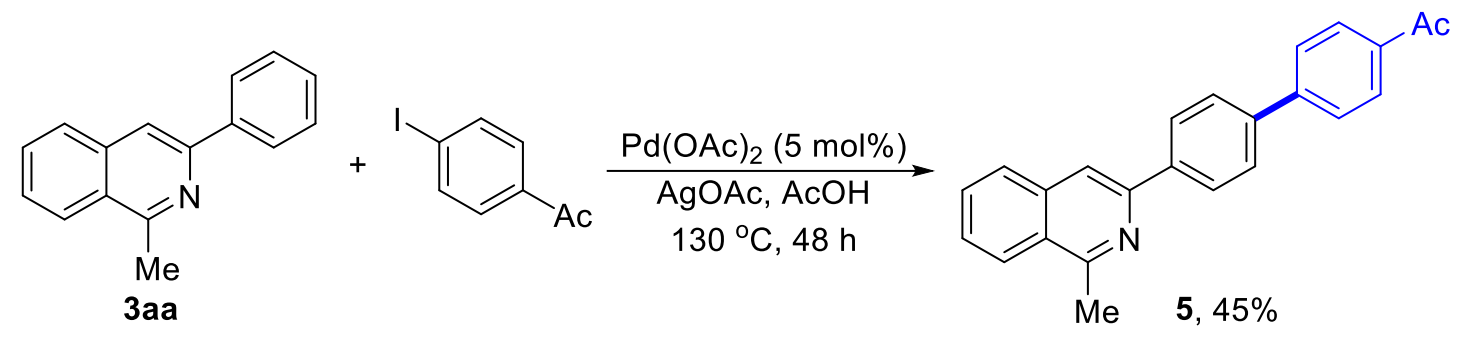

To an oven-dried Schlenk tube was charged with $\mathrm{Pd}(\mathrm{OAc})_{2}(5 \mathrm{~mol} \%), \mathrm{AgOAc}$ (0.15 mmol), 4-iodoacetophenone $(0.30 \mathrm{mmol})$ and 3aa $(0.10 \mathrm{mmol})$ under argon. $\mathrm{AcOH}(1.0 \mathrm{~mL})$ was added and the resulting solution was stirred in an oil-bath at $130{ }^{\circ} \mathrm{C}$ under argon for 48 hours. After completion of the reaction, ethyl acetate was added to the reaction mixture followed by filtration through a pad of Celite. The filtrated was evaporated under reduced pressure and residue was purified by column chromatography on silica gel to give analytically pure product 5 in $45 \%$ yield.

\section{1-(4'-(1-Methylisoquinolin-3-yl)-[1,1'-biphenyl]-4-yl)ethan-1-one (5)}

Obtained as a white solid, m.p. 104.1-105.4 ${ }^{\circ} \mathrm{C}$.

Eluent: Petroleum ether/ethyl acetate 10:1; isolated yield 45\% (15.2 mg from 21.9 mg of 3aa).

${ }^{1}$ H NMR (600 MHz, $\left.\mathbf{C D C l}_{3}\right) \delta 8.08(\mathrm{~d}, J=8.22 \mathrm{~Hz}, 1 \mathrm{H}), 7.80-7.77(\mathrm{~m}, 3 \mathrm{H})$, 7.61-7.45 (m, $6 \mathrm{H}), 7.31$ (d, J = 8.16 Hz, $2 \mathrm{H}), 7.26$ (s, $1 \mathrm{H}), 2.89$ (s, $3 \mathrm{H}), 2.53$ (s, $3 \mathrm{H})$.

${ }^{13}$ C NMR (100 MHz, CDCl 3$) \delta 197.9,158.3,147.0,139.8,136.0,135.1,131.0$, 130.4, 130.3, 129.9, 128.4, 128.3, 128.0, 127.4, 127.1, 125.9, 125.6, 120.3, 26.5, 21.9.

FT-IR (KBr, cm$\left.^{-1}\right) \tilde{v}_{\max }$ 3066, 2960, 2920, 1680, 1600, 1562, 1490, 1433, 1399, 1390, 1356, 1330, 1264, 1183, 1141, 1104, 1022, 957, 904, 882, 836, 793, 769, 752, 
$731,688,598,520$.

HR-ESI-MS: $[\mathrm{M}+\mathrm{H}]^{+} \mathrm{m} / \mathrm{z}$ calcd for $\mathrm{C}_{24} \mathrm{H}_{20} \mathrm{NO}, 338.1539$; found, 338.1537 .

\section{General procedure for the oxidation of 3aa with $\mathrm{H}_{2} \mathrm{O}_{2}{ }^{[16]}$}<smiles></smiles>

$\stackrel{\mathrm{H}_{2} \mathrm{O}_{2}, \mathrm{AcOH}, 70^{\circ} \mathrm{C}, 72 \mathrm{~h}}{\longrightarrow}$<smiles>O=[N+]([O-])c1c2c([N+]([O-])[O-])cccc2cc(-c2ccccc2)[n+]1[O-]</smiles>

Hydrogen peroxide ( $30 \%$ aqueous solution, $0.14 \mathrm{~mL}$ ) was added into the solution of $3 \mathbf{a a}(1.0 \mathrm{mmol})$ in acetic acid $(1.0 \mathrm{~mL})$. The reaction mixture was stirred in an oilbath at $70{ }^{\circ} \mathrm{C}$ for 72 hours. After completion of the reaction, the reaction mixture was evaporated under vacuum, and the residue was basified with aqueous solution of sodium carbonate to $p \mathrm{H}=9$. The resulting mixture was extracted with $\mathrm{CHCl}_{3}(10 \mathrm{~mL}$ $\times 3$ ). The organic layer was combined and dried over anhydrous $\mathrm{Na}_{2} \mathrm{SO}_{4}$, filtered and evaporated under vacuum. The residue was purified by column chromatography on silica gel to give analytically pure product 6 in $90 \%$ yield.

\section{1-Methyl-3-phenylisoquinoline 2-oxide (6)}

Obtained as a light yellow solid, m.p. $150.2-153.7^{\circ} \mathrm{C}$.

Eluent: ethyl acetate/methanol 10:1; isolated yield 90\% (211.8 mg from $219.3 \mathrm{mg}$ of 3aa).

${ }^{1}$ H NMR (600 MHz, CDCl $) \delta 7.96(\mathrm{~d}, J=8.52 \mathrm{~Hz}, 1 \mathrm{H}), 7.78-7.77(\mathrm{~m}, 3 \mathrm{H})$, 7.69 (s, $1 \mathrm{H}), 7.63$ (t, $J=7.65 \mathrm{~Hz}, 1 \mathrm{H}), 7.56$ (t, $J=7.44,1 \mathrm{H}) .7 .49-7.45$ (m, $3 \mathrm{H}), 2.96$ (s, $3 \mathrm{H})$.

${ }^{13}$ C NMR (100 MHz, CDCl $) \delta$ 146.7, 146.4, 133.6, 129.8, 129.1, 128.9, 128.6, $128.4,128.2,128.0,127.4,123.9,122.6,13.6$.

FT-IR (KBr, $\left.\mathbf{~ c m}^{-1}\right) \tilde{v}_{\max } 3051,3031,2923,1944,1628,1592,1554,1496,1442$, 1336, 1307, 1288, 1220, 1135, 1066, 1022, 989, 962, 887, 819, 766, 745, 719, 689, 621, 578.

HR-ESI-MS: $[\mathrm{M}+\mathrm{H}]^{+} \mathrm{m} / \mathrm{z}$ calcd for $\mathrm{C}_{16} \mathrm{H}_{14} \mathrm{NO}, 236.1070$; found, 236.1068. 
X-ray structure data for 6 :

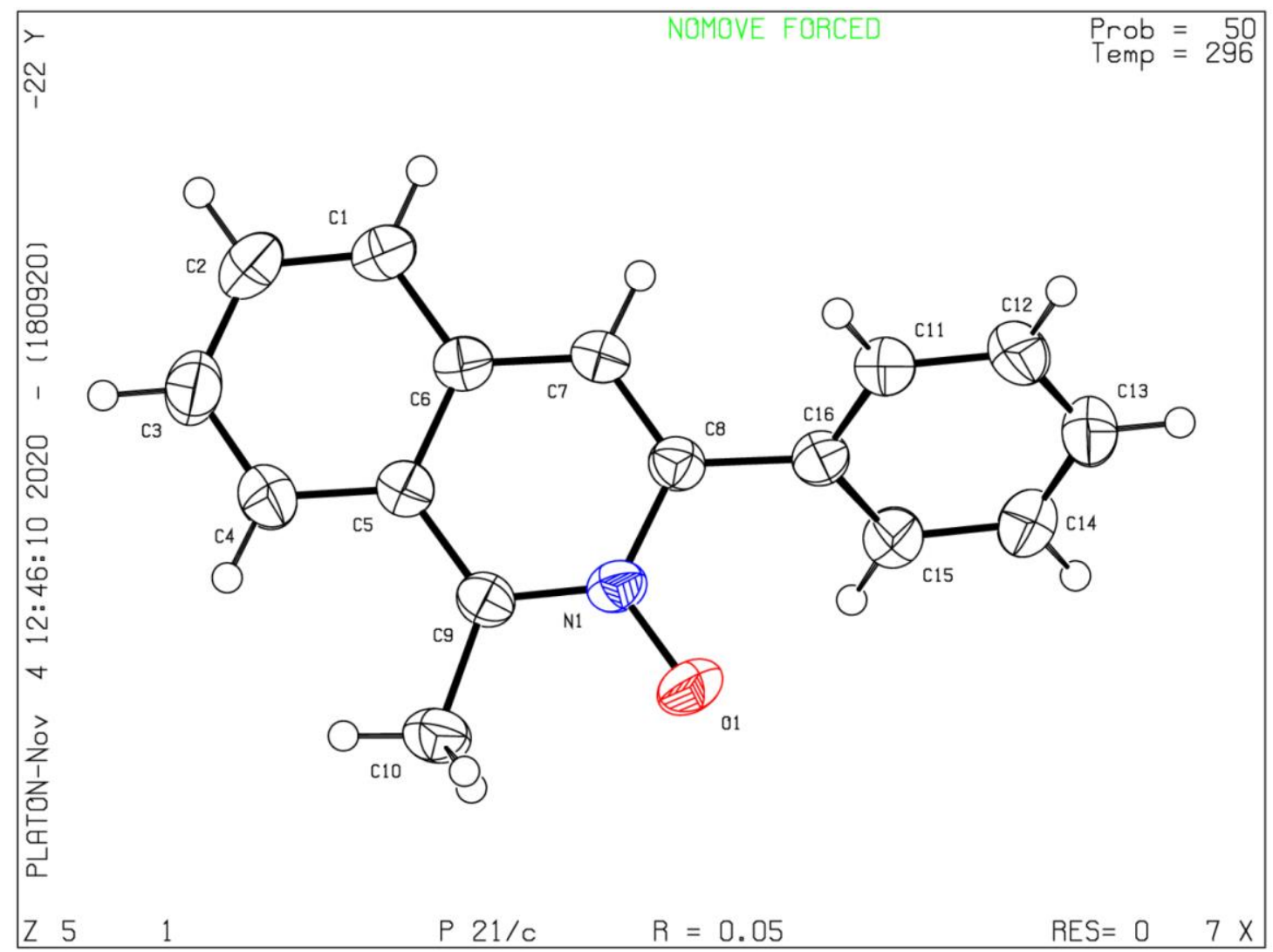

\section{Crystal data and structure refinement:}

Identification code

Empirical formula

Formula weight

Temperature

Wavelength

Crystal system, space group

Unit cell dimensions

Volume

Z

Calculated density

Absorption coefficient

$\mathrm{F}(000)$

\section{1 \\ C16H13NO \\ 235.27 \\ 296(2) K \\ $0.71073 \AA$}

Monoclinic, P2(1)/c

$\mathrm{a}=11.1805(6) \AA \quad \alpha=90 \mathrm{deg}$.

$\mathrm{b}=14.6987(8) \AA \quad \beta=99.457(2) \mathrm{deg}$.

$\mathrm{c}=7.2618(4) \AA \quad \gamma=90 \mathrm{deg}$.

$1177.18(11) \AA^{3}$

4

$1.328 \mathrm{Mg} / \mathrm{m}^{3}$

$0.083 \mathrm{~mm}^{-1}$

496 
Crystal size

Theta range for data collection

Limiting indices

Reflections collected / unique

Completeness to theta $=25.242$

Refinement method

Data / restraints / parameters

Goodness-of-fit on F2

Final R indices [I $>2 \operatorname{sigma}(\mathrm{I})]$

$\mathrm{R}$ indices (all data)

Extinction coefficient

Largest diff. peak and hole
$0.200 \times 0.150 \times 0.100 \mathrm{~mm}$

1.847 to $26.482^{\circ}$.

$-14<=\mathrm{h}<=13,-18<=\mathrm{k}<=18,-9<=\mathrm{l}<=9$

$12513 / 2412[\mathrm{R}(\mathrm{int})=0.2077]$

$100.0 \%$

Full-matrix least-squares on $\mathrm{F}^{\wedge} 2$

2412 / 0 / 163

1.063

$\mathrm{R} 1=0.0559, \mathrm{wR} 2=0.1564$

$\mathrm{R} 1=0.0676, \mathrm{wR} 2=0.1673$

$\mathrm{n} / \mathrm{a}$

0.238 and -0.290 e. $\AA^{-3}$

\section{General procedure for the oxidation of 3aa with $\mathrm{SeO}_{2}{ }^{[11]}$}<smiles>Cc1nc(-c2ccccc2)cc2ccccc12</smiles>

3aa

$$
\frac{\mathrm{SeO}_{2}, p \text {-dioxane }}{\text { air, } 102^{\circ} \mathrm{C}, 3 \mathrm{~h}}
$$<smiles>O=[W]OCc1nc(-c2ccccc2)cc2ccccc12</smiles>

An oven-dried Schlenk tube was charged with 3aa $(0.56 \mathrm{mmol})$ and $\mathrm{SeO}_{2}(0.68$ mmol) in anhydrous $p$-dioxane $(10.5 \mathrm{~mL})$, sealed and heated in an oil bath at $102{ }^{\circ} \mathrm{C}$ under air for 3 hours. The resulting reaction mixture was then cooled to room temperature and filtered through a pad of Celite to remove the precipitate. The resulting filtrate was concentrated under vacuum and the residue was purified by chromatography on silica gel to give analytically pure product 7 in $90 \%$ yield.

\section{Phenylisoquinoline-1-carbaldehyde (7) ${ }^{[11]}$}

Obtained as a white solid, m.p. $98-99.5^{\circ} \mathrm{C}$.

Eluent: Petroleum ether/ethyl acetate 100:1; isolated yield 90\% (117.8 mg from $122.8 \mathrm{mg}$ of 3aa).

${ }^{1}$ H NMR (600 MHz, CDCl3) $\delta 10.49$ (s, 1 H), $9.32(\mathrm{~d}, J=8.82 \mathrm{~Hz}, 1 \mathrm{H}), 8.29$ (s, 
$1 \mathrm{H}), 8.24(\mathrm{~d}, J=7.02 \mathrm{~Hz}, 2 \mathrm{H}), 7.96(\mathrm{~d}, J=7.44 \mathrm{~Hz}, 1 \mathrm{H}), 7.76(\mathrm{td}, J=7.46,1.32 \mathrm{~Hz}$,

$1 \mathrm{H}), 7.73(\mathrm{td}, J=7.59,1.56 \mathrm{~Hz}, 1 \mathrm{H}), 7.56(\mathrm{t}, J=7.68 \mathrm{~Hz}, 2 \mathrm{H}), 7.47(\mathrm{t}, J=7.35,1 \mathrm{H})$.

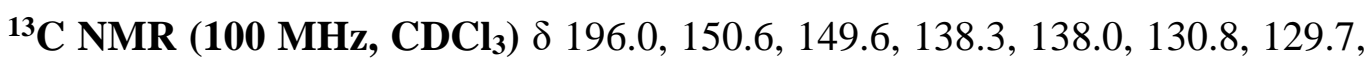
$129.1,129.0,127.3,126.9,125.8,125.3,121.1$.

FT-IR (KBr, cm') $\tilde{v}_{\max }$ 3033, 2961, 2923, 2822, 1704, 1618, 1573, 1491, 1449, 1331, 1261, 1137, 1073, 1053, 1024, 901, 804, 764, 718, 683, 644, 568, 537, 505.

HR-ESI-MS: [M+H $]^{+} \mathrm{m} / \mathrm{z}$ calcd for $\mathrm{C}_{16} \mathrm{H}_{12} \mathrm{NO}, 234.0913$; found, 234.0912.

\section{General procedure for CDC reaction of 3aa with phthalimide ${ }^{[17]}$}<smiles></smiles>

An oven-dried Schlenk tube was charged with 3aa $(0.10 \mathrm{mmol}),{ }^{t} \mathrm{BuOK}(0.001$ mol\%, dissolved in benzene), benzene $(0.5 \mathrm{~mL}), \mathrm{CuCl}(10 \mathrm{~mol} \%)$ and DTBP $(0.20$ mmol) under argon. The tube was then sealed and stirred in an oil-bath at $120{ }^{\circ} \mathrm{C}$ under argon for 24 hours. After the reaction mixture was cooled to room temperature, the reaction mixture was extracted with ethyl acetate $(10 \mathrm{~mL} \times 3)$, dried over anhydrous $\mathrm{MgSO}_{4}$, filtered and concentrated in vacuo. And the resulting mixture was purified by column chromatography on silica gel to give analytically pure product $\mathbf{8}$ in $27 \%$ yield.

\section{2-((3-Phenylisoquinolin-1-yl)methyl)isoindoline-1,3-dione (8)}

Obtained as a light-yellow solid, m.p. $187-189.2{ }^{\circ} \mathrm{C}$.

Eluent: Petroleum ether/ethyl acetate 100:1; isolated yield 27\% (9.9 mg from 21.9 mg of 3aa).

${ }^{1}$ H NMR (600 MHz, $\left.\mathbf{C D C l}_{3}\right) \delta 8.19(\mathrm{~d}, J=8.28 \mathrm{~Hz}, 1 \mathrm{H}), 7.97-7.51(\mathrm{~m}, 3 \mathrm{H})$, $7.88(\mathrm{~d}, J=8.22 \mathrm{~Hz}, 1 \mathrm{H}), 7.79-7.78(\mathrm{~m}, 4 \mathrm{H}), 7.70(\mathrm{t}, J=7.50 \mathrm{~Hz}, 1 \mathrm{H}), 7.63(\mathrm{t}, J=$ 7.62, $1 \mathrm{H}), 7.23-7.21(\mathrm{~m}, 3 \mathrm{H}), 5.62(\mathrm{~s}, 2 \mathrm{H})$. 
${ }^{13}$ C NMR (100 MHz, CDCl $) \delta$ 168.8, 152.2, 149.0, 138.7, 137.3, 133.9, 132.7, $130.4,128.4,128.3,127.9,127.3,126.5,124.9,123.7,123.4,116.0,40.9$.

FT-IR (KBr, cm $\left.{ }^{-1}\right) \tilde{V}_{\max }$ 3464, 3060, 2962, 2924, 2854, 1767, 1712, 1620, 1571, $1498,1465,1421,1375,1317,1261,1189,1098,1020,942,885,857,800,747,723$, $690,601,572,528,507$.

HR-ESI-MS: $[\mathrm{M}+\mathrm{H}]^{+} \mathrm{m} / \mathrm{z}$ calcd for $\mathrm{C}_{24} \mathrm{H}_{17} \mathrm{~N}_{2} \mathrm{O}_{2}, 365.1285$; found, 365.1279 .

\section{General procedure for the conversion of 3aa to nitrile ${ }^{[18]}$}

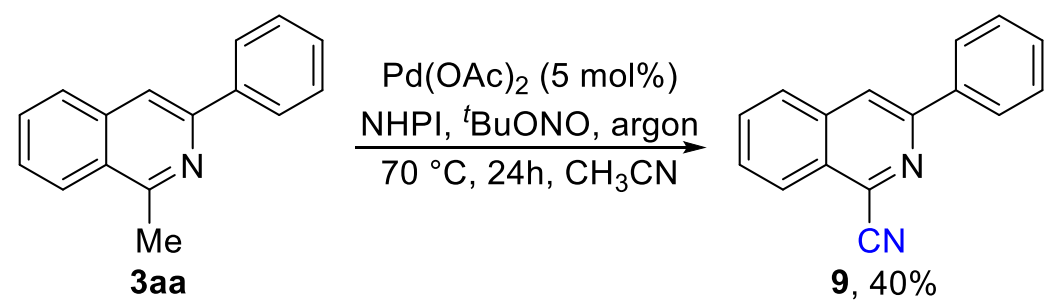

To an oven-dried Schlenk tube was charged with 3aa $(0.10 \mathrm{mmol}),{ }^{t} \mathrm{BuONO}(0.20$ $\mathrm{mmol})$, NHPI (0.03 mmol), Pd(OAc) $2(5 \mathrm{~mol} \%)$ and acetonitrile $(0.20 \mathrm{~mL})$ under argon. The reaction mixture was stirred in an oil-bath at $70{ }^{\circ} \mathrm{C}$ for 24 hours. After cooling to temperature, the reaction was concentrated under vacuum, and the residue was purified by column chromatography on silica gel to give analytically pure product 9 in $40 \%$ yield.

\section{2-phenylisoquinoline-1-carbonitrile (9) ${ }^{[19]}$}

Obtained as a white solid, m.p. $136.2-137.9^{\circ} \mathrm{C}$.

Eluent: Petroleum ether/ethyl acetate 100:1; isolated yield 40\% (9.3 mg from 21.9 mg of 3aa).

${ }^{1}$ H NMR (600 MHz, CDCl $) \delta 8.34(\mathrm{~d}, J=7.26 \mathrm{~Hz}, 1 \mathrm{H}), 8.29(\mathrm{~s}, 1 \mathrm{H}), 8.15$ (d, $J=7.02 \mathrm{~Hz}, 2 \mathrm{H}), 7.98(\mathrm{~d}, J=8.22 \mathrm{~Hz}, 1 \mathrm{H}), 7.81(\mathrm{t}, J=6.99 \mathrm{~Hz}, 1 \mathrm{H}), 7.76(\mathrm{t}, J=7.32$ $\mathrm{Hz}, 1 \mathrm{H}), 7.53(\mathrm{t}, J=7.65 \mathrm{~Hz}, 2 \mathrm{H}), 7.47$ (t, $J=7.32,1 \mathrm{H})$.

${ }^{13}$ C NMR (100 MHz, CDCl $) \delta$ 151.9, 137.6, 137.0, 134.6, 131.8, 129.49, 129.47, $129.0,128.4,127.7,127.0,125.3,120.0,116.0$.

FT-IR (KBr, cm$\left.^{-1}\right) \tilde{V}_{\max } 3058,2923,2361,2228,1620,1570,1492,1451,1406$, $1376,1329,1285,1261,1027,896,857,787,755,678,570,519$. 
HR-ESI-MS: $[\mathrm{M}+\mathrm{H}]^{+} \mathrm{m} / \mathrm{z}$ calcd for $\mathrm{C}_{16} \mathrm{H}_{11} \mathrm{~N}_{2}, 231.0917$; found, 231.0917 .

\section{General procedure for the synthesis of CWJ-a-5 from 3aa ${ }^{[20]}$}

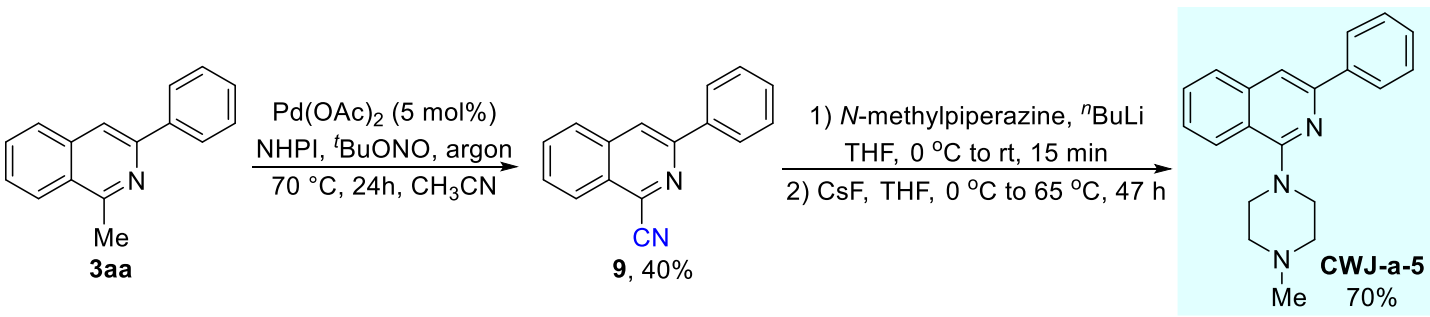

To an oven-dried Schlenk tube was charged with $N$-methylpiperazine $(0.40 \mathrm{mmol})$ and anhydrous THF $(1.0 \mathrm{~mL})$ under argon. The mixture was cooled to $0{ }^{\circ} \mathrm{C}$ in an ice bath, and treated with ${ }^{n} \mathrm{BuLi}(0.16 \mathrm{~mL}, 0.40 \mathrm{mmol} ; 2.5 \mathrm{M}$ in hexane) under argon. Then the mixture was allowed to warm to room temperature for 15 minutes, which was subsequently cooled to $0{ }^{\circ} \mathrm{C}$. A solution of $9(0.20 \mathrm{mmol}$; obtained from 3aa via palladium catalysis $)$ in anhydrous THF $(0.5 \mathrm{~mL})$ was carefully added under argon. After warming to room temperature, the entire reaction mixture was added $\mathrm{CsF}(0.30 \mathrm{mmol})$ and heated to $65^{\circ} \mathrm{C}$ in an oil-bath under argon for 47 hours. After cooling to temperature, the mixture was concentrated under vacuum and extracted with $\mathrm{CHCl}_{3}(15 \mathrm{~mL} \times 3)$, washed with aqueous $\mathrm{NH}_{4} \mathrm{Cl}$ solution and saturated brine, dried over $\mathrm{Na}_{2} \mathrm{SO}_{4}$ and concentrated under vacuum. The residue was purified by column chromatography on silica gel to give analytically pure $\mathbf{C W J}-\mathbf{a}-5$ in $70 \%$ yield.

\section{1-(4-Methylpiperazin-1-yl)-3-phenylisoquinoline (CWJ-a-5) ${ }^{[21]}$}<smiles>CN1CCN(c2nc(-c3ccccc3)cc3ccccc23)CC1</smiles>

Obtained as a pale-yellow solid, m.p. $217.0-218.1^{\circ} \mathrm{C}$.

Eluent: dichloromethane/methanol 100:1; isolated yield 70\% (42.6 mg from 46.1 mg of 9).

${ }^{1}$ H NMR (600 MHz, CDCl $) \delta 8.16(\mathrm{~d}, J=6.96 \mathrm{~Hz}, 2 \mathrm{H}), 8.05(\mathrm{~d}, J=8.34 \mathrm{~Hz}, 1$ 
H), $7.80(\mathrm{~d}, J=8.16 \mathrm{~Hz}, 1 \mathrm{H}), 7.72(\mathrm{~s}, 1 \mathrm{H}), 7.60(\mathrm{td}, J=7.56,1.20 \mathrm{~Hz}, 1 \mathrm{H}), 7.48(\mathrm{t}, J$

$=7.68 \mathrm{~Hz}, 3 \mathrm{H}), 7.38(\mathrm{t}, J=7.35 \mathrm{~Hz}, 1 \mathrm{H}), 3.70(\mathrm{t}, J=4.62 \mathrm{~Hz}, 4 \mathrm{H}), 2.87(\mathrm{t}, J=4.74$ $\mathrm{Hz}, 4 \mathrm{H}), 2.51(\mathrm{~s}, 3 \mathrm{H})$.

${ }^{13}$ C NMR (100 MHz, CDCl $) \delta$ 160.1, 148.2, 139.5, 139.1, 129.7, 128.6, 128.3, $127.7,126.6,125.9,125.3,120.5,111.4,54.7,50.3,45.7$.

FT-IR (KBr, cm$\left.^{-1}\right) \tilde{V}_{\max }$ 3425, 3058, 3032, 2904, 2844, 2545, 2509, 2337, 1616, 1590, 1562, 1496, 1456, 1395, 1367, 1343, 1277, 1204, 1184, 1147, 1116, 1091, 1058, $1025,986,939,853,804,773,694,533$.

HR-ESI-MS: $[\mathrm{M}+\mathrm{H}]^{+} \mathrm{m} / \mathrm{z}$ calcd for $\mathrm{C}_{20} \mathrm{H}_{22} \mathrm{~N}_{3}, 304.1808$; found, 304.1801.

\section{General procedure for the synthesis of pyrimidines}
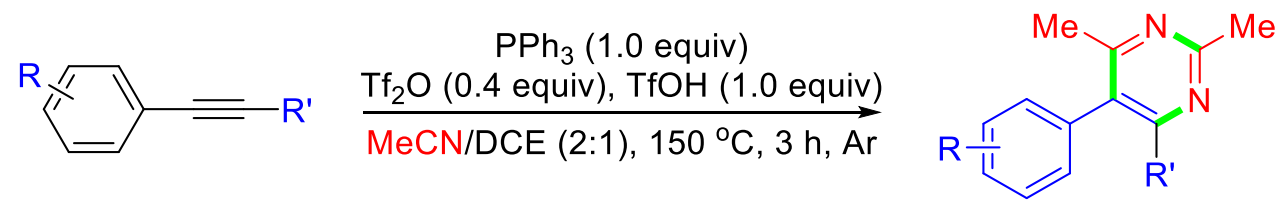

An oven-dried Schlenk tube was charged with alkynes $(0.10 \mathrm{mmol})$, triphenylphosphine $(26.2 \mathrm{mg}, 0.10 \mathrm{mmol})$. After the tube was filled with argon, acetonitrile $(0.4 \mathrm{~mL})$ and 1,2-dichloroethane $(0.2 \mathrm{~mL})$ were added by a syringe. Then the mixture was stirred at room temperature, and trifluoromethanesulfonic anhydride $(6 \mathrm{uL}, 0.04 \mathrm{mmol})$ and trifluoromethanesulfonic acid $(8.9 \mathrm{uL}, 0.10 \mathrm{mmol})$ were added sequentially by a syringe under argon. The reaction mixture was sealed and heated at $150{ }^{\circ} \mathrm{C}$ in an oil bath for 3 hours. Upon completion, the resulting mixture was cooled to room temperature, and triethylamine $(0.5 \mathrm{ml})$ was added by a syringe. The resulting solution was stirred for more than 1 hour under air, filtered through a short silica gel pad and washed with ethyl acetate. The above solution was evaporated under vacuum, and the residue was purified by silica gel column with petroleum ether/acetone as the eluent to give the analytically pure product 10-13.

2,4-Dimethyl-5-phenylpyrimidine $(10)^{[22]}$ 


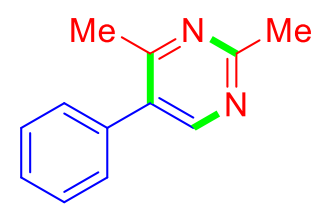

Obtained as a yellow oil.

Eluent: petroleum ether/acetone 100:1; isolated yield $78 \%$ (14.4 mg from $10.3 \mathrm{mg}$ of phenylacetylene).

${ }^{1}$ H NMR (600 MHz, CDCl $)$ ) 8 8.05-8.03 (m, 2 H), 7.48-7.47 (m, 3 H), $7.36(\mathrm{~s}, 1$ H), $2.76(\mathrm{~s}, 3 \mathrm{H}), 2.54(\mathrm{~s}, 3 \mathrm{H})$.

${ }^{13}$ C NMR (100 MHz, CDCl $\left._{3}\right) \delta 167.9,167.3,164.0,137.2,130.6,128.9,127.2$, $113.4,26.2,24.3$.

FT-IR (KBr, $\left.\mathbf{c m}^{-1}\right) \tilde{V}_{\max } 3063,2962,2924,1584,1534,1496,1441,1396,1379$, 1356, 1262, 1211, 1074, 1030, 858, 789, 749, 693, 655, 631, 587, 546.

HR-ESI-MS: $[\mathrm{M}+\mathrm{H}]^{+} \mathrm{m} / \mathrm{z}$ calcd for $\mathrm{C}_{12} \mathrm{H}_{13} \mathrm{~N}_{2}$ 185.1073; found 185.1072.

\section{5-(4-tert-Butylphenyl)-2,4-dimethylpyrimidine (11)}<smiles>Cc1ncc(-c2ccc(C(C)(C)C)cc2)c(C)n1</smiles>

Obtained as a yellow oil.

Eluent: petroleum ether/acetone 100:1; isolated yield $92 \%(22.2 \mathrm{mg}$ from $15.9 \mathrm{mg}$ of 4-tert-butylphenylacetylene).

${ }^{1}$ H NMR $\left(600 \mathrm{MHz}, \mathbf{C D C l}_{3}\right) \delta 7.98(\mathrm{~d}, J=8.46 \mathrm{~Hz}, 2 \mathrm{H}), 7.50(\mathrm{~d}, J=8.52 \mathrm{~Hz}, 2$ H), $7.34(\mathrm{~s}, 1 \mathrm{H}), 2.75(\mathrm{~s}, 3 \mathrm{H}), 2.54(\mathrm{~s}, 3 \mathrm{H}), 1.35$ (s, $9 \mathrm{H})$.

${ }^{13}$ C NMR (100 MHz, CDCl 3$) \delta 167.8,167.0,164.0,154.0,134.4,127.0,125.9$, 113.2, 34.9, 31.2, 26.2, 24.3.

FT-IR (KBr, $\left.\mathbf{~ c m}^{-1}\right) \tilde{V}_{\max } 3066,2963,2924,2868,1586,1537,1445,1380,1358$, $1267,1200,1118,1022,864,838,772,721,741,715,632,593,546$.

HR-ESI-MS: [M+H] $]^{+} \mathrm{m} / \mathrm{z}$ calcd for $\mathrm{C}_{16} \mathrm{H}_{21} \mathrm{~N}_{2}$ 241.1699; found 241.1699. 


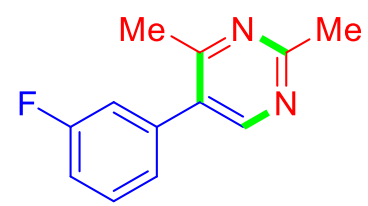

Obtained as a light yellow solid, m.p. $41.0-43.3{ }^{\circ} \mathrm{C}$.

Eluent: petroleum ether/acetone 100:1; isolated yield 66 \% (13.4 mg from $12.0 \mathrm{mg}$ of 3-fluorophenlyacetylene).

${ }^{1}$ H NMR (600 MHz, CDCl $) \delta$ 7.82-7.78 (m, $\left.2 \mathrm{H}\right), 7.45(\mathrm{td}, J=8.00,5.82 \mathrm{~Hz}, 1$ H), 7.35 (s, $1 \mathrm{H}), 7.19-7.16(\mathrm{~m}, 1 \mathrm{H}), 2.76$ (s, $3 \mathrm{H}), 2.56$ (s, $3 \mathrm{H})$.

${ }^{13}$ C NMR (100 MHz, CDCl $) \delta 168.1,167.6,163.3\left(\mathrm{C}-\mathrm{F},{ }^{1} J_{C-F}=244.9 \mathrm{~Hz}\right), 162.7$ $\left(\mathrm{C}-\mathrm{F},{ }^{4} J_{C-F}=2.6 \mathrm{~Hz}\right), 139.5\left(\mathrm{C}-\mathrm{F},{ }^{3} J_{C-F}=7.4 \mathrm{~Hz}\right), 130.4\left(\mathrm{C}-\mathrm{F},{ }^{3} J_{C-F}=8.0 \mathrm{~Hz}\right), 122.7(\mathrm{C}-$ $\left.\mathrm{F},{ }^{4} J_{C-F}=3.0 \mathrm{~Hz}\right), 117.5\left(\mathrm{C}-\mathrm{F},{ }^{2} J_{C-F}=21.2 \mathrm{~Hz}\right), 114.2\left(\mathrm{C}-\mathrm{F},{ }^{2} J_{C-F}=22.8 \mathrm{~Hz}\right), 113.5,26.2$, 24.3.

FT-IR (KBr, $\left.\mathbf{~ c m}^{-1}\right) \tilde{V}_{\max } 3082,3047,3013,2963,2924,2853,1582,1533,1489$, $1439,1398,1373,1344,1265,1237,1194,1167,1088,1029,994,924,891,859,793$, $762,688,649,585,549,505$.

HR-ESI-MS: $[\mathrm{M}+\mathrm{H}]^{+} \mathrm{m} / \mathrm{z}$ calcd for $\mathrm{C}_{12} \mathrm{H}_{12} \mathrm{FN}_{2}$, 203.0979; found 203.0979.

\section{2,4,6-Trimethyl-5-phenylpyrimidine (13)}

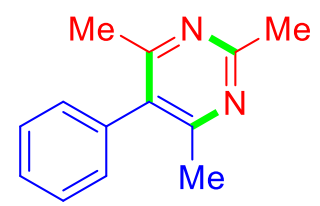

Obtained as a white solid, m.p. $55.5-58.5^{\circ} \mathrm{C}$.

Eluent: petroleum ether/acetone 100:1; isolated yield $82 \%$ (16.4 mg from $11.7 \mathrm{mg}$ of 1-phenyl-1-propyne).

${ }^{1}$ H NMR (600 MHz, CDCl $) \delta 7.49(\mathrm{~d}, J=6.72 \mathrm{~Hz}, 2 \mathrm{H}), 7.46-7.40(\mathrm{~m}, 3 \mathrm{H}), 2.70$ (s, $3 \mathrm{H}), 2.53(\mathrm{~s}, 3 \mathrm{H}), 2.23(\mathrm{~s}, 3 \mathrm{H})$.

${ }^{13}$ C NMR (100 MHz, CDCl $\left.\mathbf{3}\right) \delta 166.4,165.1,164.3,138.8,128.9,128.8,128.3$, $123.0,25.7,22.8,15.2$.

FT-IR (KBr, $\left.\mathbf{c m}^{-1}\right) \tilde{v}_{\max }$ 3054, 2959, 2923, 2857, 1582, 1492, 1448, 1431, 1409, 1376, 1337, 1181, 1079, 1012, 945, 842, 797, 768, 748, 706, 649, 577, 556.

HR-ESI-MS: $[\mathrm{M}+\mathrm{H}]^{+} \mathrm{m} / \mathrm{z}$ calcd for $\mathrm{C}_{13} \mathrm{H}_{15} \mathrm{~N}_{2}, 199.1223$; found 199.1229 . 


\section{Mechanism studies}

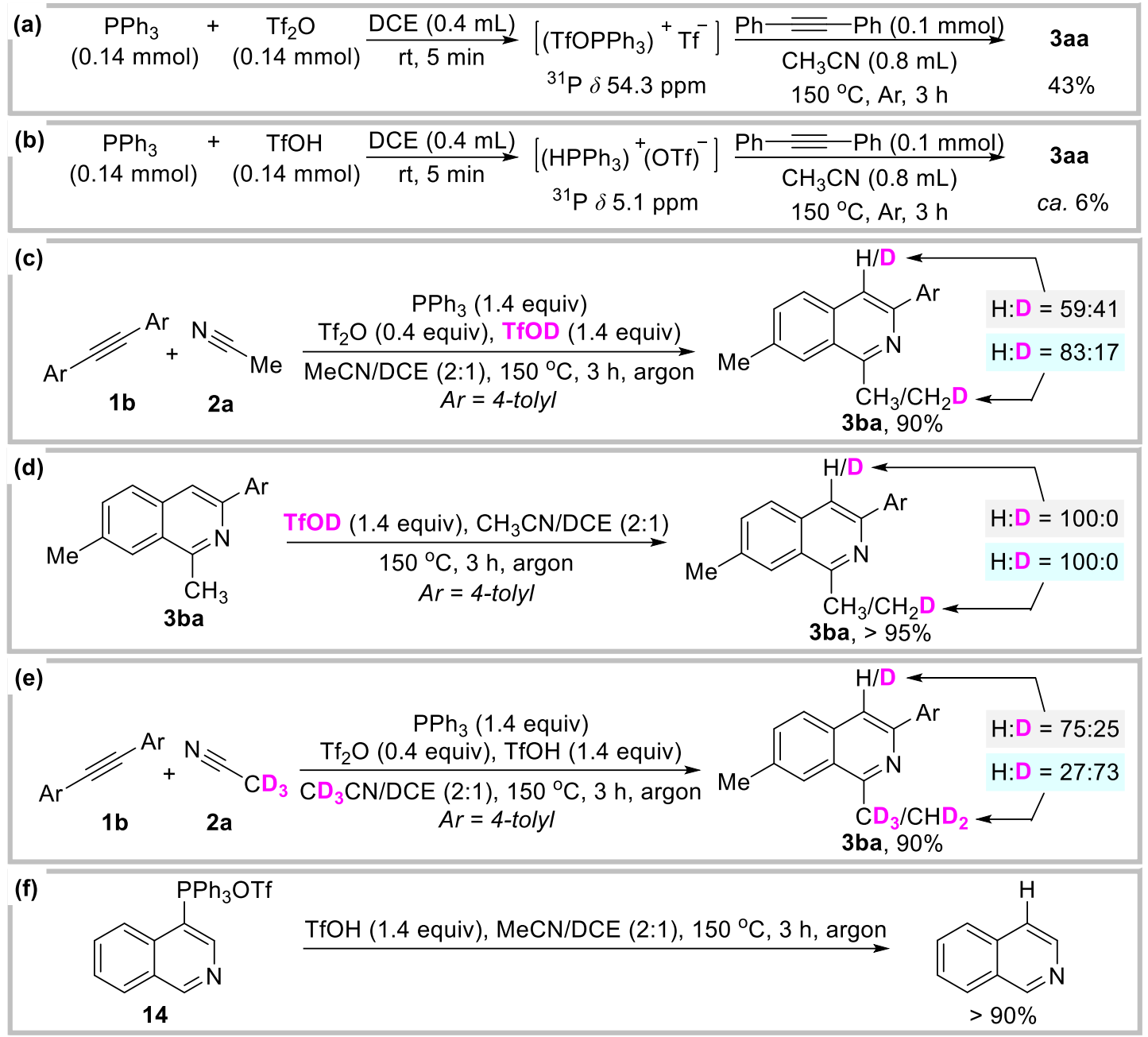

Scheme S2. Overview of mechanistic studies.

\section{A. Exploration of the crucial phosphonium species with ${ }^{31} \mathrm{P}-\mathrm{NMR}$}

\section{Step 1:}

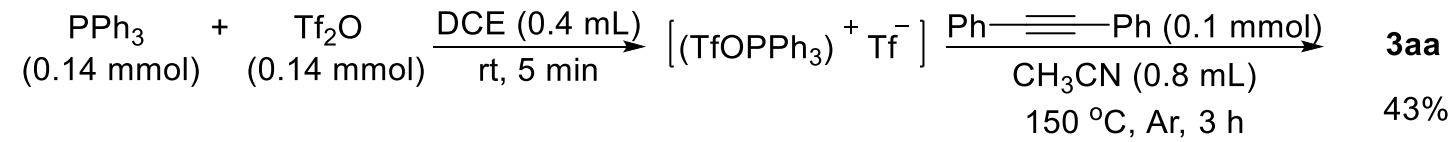

To an oven-dried Schlenk tube was charged with $\mathrm{PPh}_{3}\left(0.14\right.$ mmol), $\mathrm{Tf}_{2} \mathrm{O}(0.14$ mmol) and DCE $(0.4 \mathrm{~mL})$ under argon. The solution was stirred at room temperature under argon for 5 minutes. ${ }^{31} \mathrm{P}-\mathrm{NMR}$ spectra shows that it mainly produces $\left(\mathrm{TfOPPh}_{3}\right)^{+}(\mathrm{Tf})^{-}$with a chemical shift of $\delta 54.3 \mathrm{ppm}$ (Figure $\left.\mathrm{S} 1-\mathrm{a}\right){ }^{[23]}$ Then biphenylacetylene $(0.10 \mathrm{mmol})$ and anhydrous $\mathrm{MeCN}(0.8 \mathrm{~mL})$ were carefully added to the above solution under argon, and the resulting solution was stirred in an oil-bath 
at $150{ }^{\circ} \mathrm{C}$ under argon for 3 hours. Crude ${ }^{1} \mathrm{H}-\mathrm{NMR}$ shows that a $43 \%$ yield of 3 aa was achieved.

This result indicates that $\left(\mathrm{TfOPPh}_{3}\right)^{+}(\mathrm{Tf})^{-}$can promote the annulation reaction highly efficiently.

\section{Step 2:}

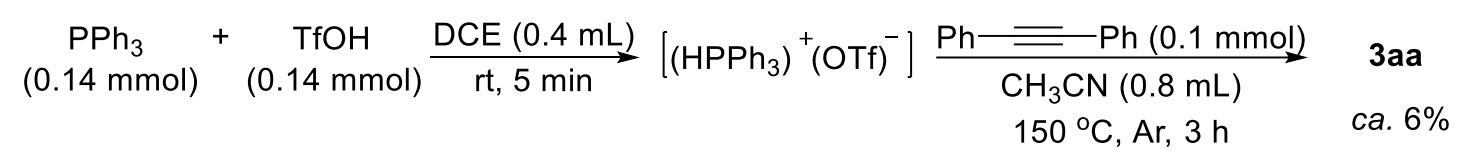

To an oven-dried Schlenk tube was charged with $\mathrm{PPh}_{3}(0.14 \mathrm{mmol}), \mathrm{TfOH}$ (0.14 $\mathrm{mmol})$ and DCE $(0.4 \mathrm{~mL})$ under argon. The solution was stirred at room temperature under argon for 5 minutes. ${ }^{31} \mathrm{P}-\mathrm{NMR}$ spectra shows that it mainly produces $\left(\mathrm{TfOPPh}_{3}\right)^{+}(\mathrm{Tf})^{-}$with a chemical shift of $\delta 5.1 \mathrm{ppm}$ (Figure $\left.\mathrm{S} 1-\mathrm{b}\right){ }^{[24]}$ Then biphenylacetylene $(0.10 \mathrm{mmol})$ and anhydrous $\mathrm{MeCN}(0.8 \mathrm{~mL})$ were carefully added to the above solution under argon, and the resulting solution was stirred in an oil-bath at $150{ }^{\circ} \mathrm{C}$ under argon for 3 hours. Crude ${ }^{1} \mathrm{H}-\mathrm{NMR}$ shows that about $6 \%$ yield of 3 aa was achieved.

This result indicates that $\left(\mathrm{HPPh}_{3}\right)^{+}(\mathrm{OTf})^{-}$is most probably not directly involved in the annulation reaction.

Step 3: To an oven-dried Schlenk tube was charged with $\mathrm{PPh}_{3}(0.14 \mathrm{mmol})$, TfOH $(0.14 \mathrm{mmol})$ and DCE $(0.4 \mathrm{~mL})$ under argon. The solution was stirred at room temperature under argon for 5 minutes. Then $\mathrm{Tf}_{2} \mathrm{O}(0.14 \mathrm{mmol})$ was carefully added to the above solution under argon, and the resulting solution was stirred in an oil-bath at $150{ }^{\circ} \mathrm{C}$ under argon. After 10 minutes, ${ }^{31} \mathrm{P}-\mathrm{NMR}$ spectra shows the appearance of a chemical shift of $53.2 \mathrm{ppm}$ (Figure S1-c), which stands for the signal of $\left(\mathrm{TfOPPh}_{3}\right)^{+}(\mathrm{Tf})^{-}$.

This result indicates that $\left(\mathrm{HPPh}_{3}\right)^{+}(\mathrm{OTf})^{-}$can be converted into $\left(\mathrm{TfOPPh}_{3}\right)^{+}(\mathrm{Tf})^{-}$ upon heating the corresponding solution to $150^{\circ} \mathrm{C}$.

In conclusion, $\left(\mathrm{TfOPPh}_{3}\right)^{+}(\mathrm{Tf})^{-}$as the phosphonium species is indispensable for the formation of 3-arylisoquinoline derivatives. 


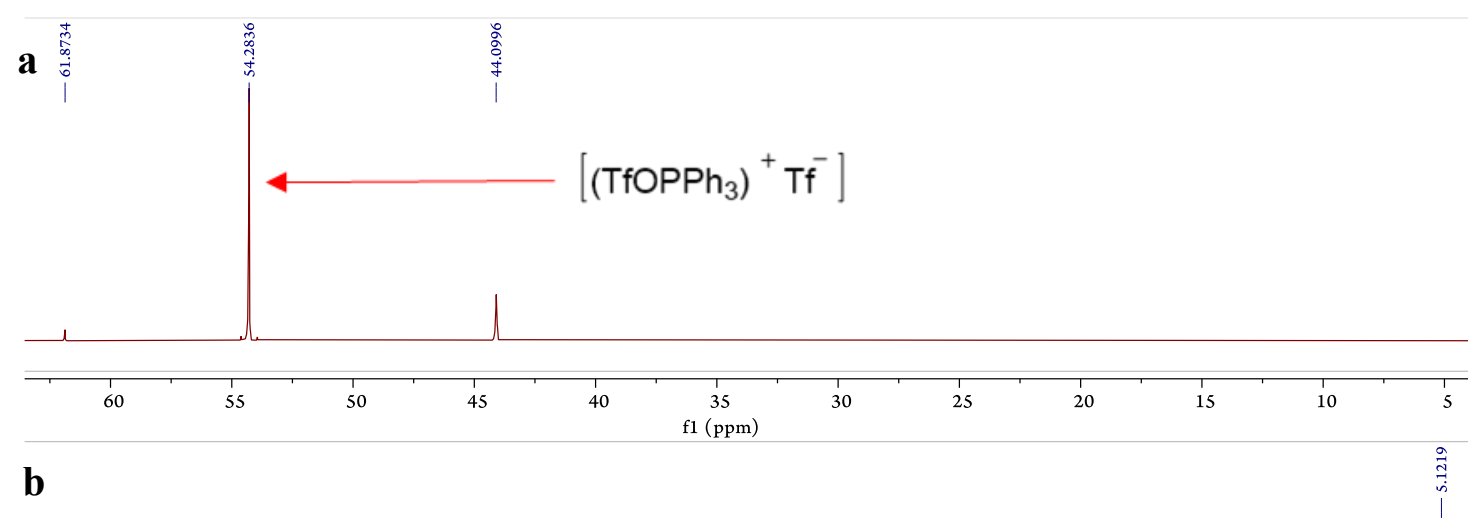

$\mathbf{b}$

$$
\left[\left(\mathrm{HPPh}_{3}\right)^{+} \mathrm{Tf}^{-}\right]
$$

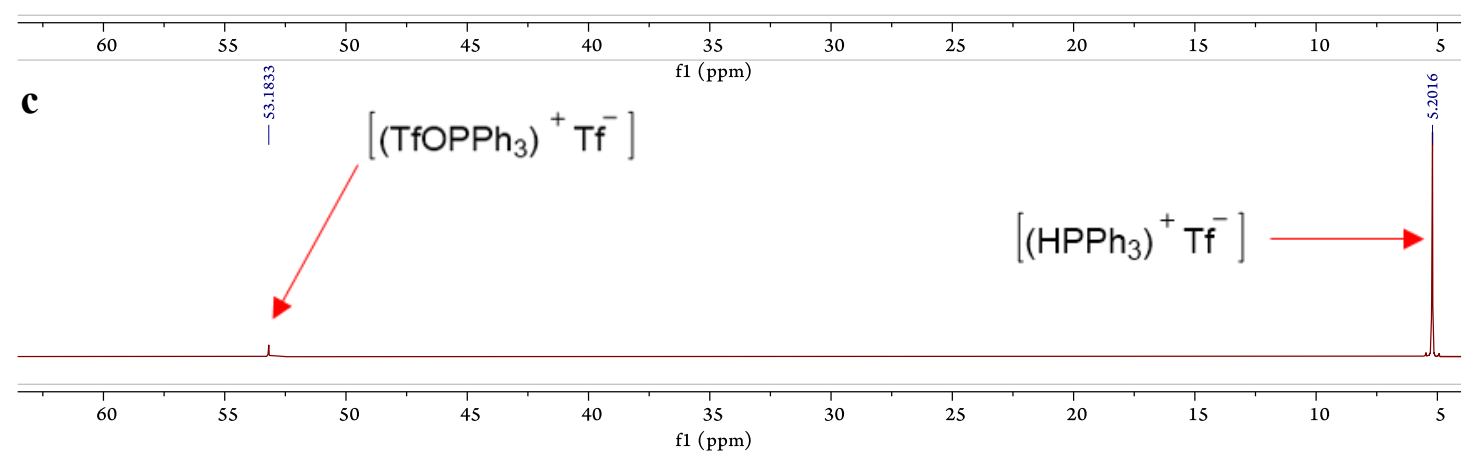

Figure S1. Determination of crucial phosphonium species with ${ }^{31}$ P-NMR: (a) Mixture of $\mathrm{PPh}_{3}$ and $\mathrm{Tf}_{2} \mathrm{O}$ in DCE at room temperature under argon. (b) Mixture of $\mathrm{PPh}_{3}$ and $\mathrm{TfOH}$ in DCE at room temperature under argon. (c) After mixing $\mathrm{PPh}_{3}$ and $\mathrm{TfOH}$ in DCE, $\mathrm{Tf}_{2} \mathrm{O}$ is added and heating the entire solution at $150^{\circ} \mathrm{C}$ under argon.

\section{B. Deuterium experiments}

\section{Step 1:}
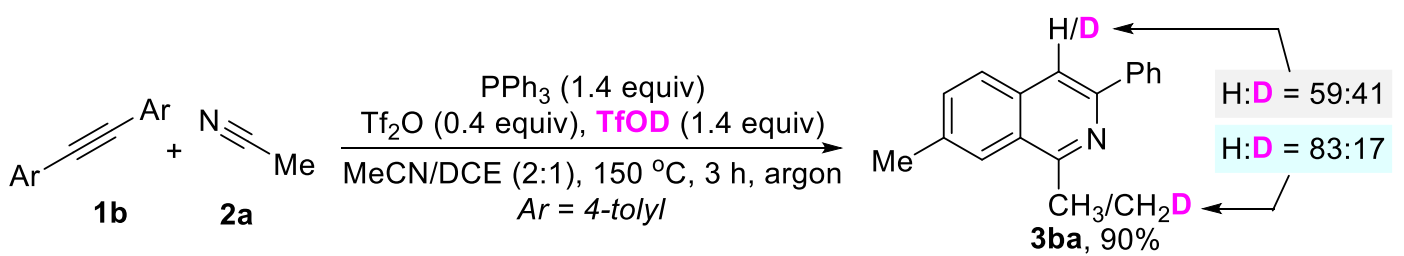

An oven-dried Schlenk tube was charged with 1,2-bis( $p$-tolyl)ethyne 1 (0.1 mmol), triphenylphosphine $(36.7 \mathrm{mg}, 0.14 \mathrm{mmol})$. After the tube was filled with argon, 1,2dichloroethane $(0.4 \mathrm{~mL})$ and acetonitrile $(0.8 \mathrm{~mL})$ were added by a syringe. Then the 
mixture was stirred at room temperature, and trifluoromethanesulfonic anhydride $(0.04$ mmol) and TfOD (0.14 mmol) were added sequentially by a syringe under argon. The reaction mixture was sealed and heated at $150{ }^{\circ} \mathrm{C}$ in an oil bath for 3 hours. Upon completion, the resulting mixture was cooled to room temperature, and triethylamine $(0.5 \mathrm{ml})$ was added by a syringe. The resulting solution was stirred for more than 1 hour under air, filtered through a short silica gel pad and washed with ethyl acetate. The above solution was evaporated under vacuum, and the residue was purified by silica gel column with petroleum ether/acetone as the eluent to give product $\mathbf{3 b a}$.

${ }^{1}$ H NMR (600 MHz, CDCl $) \delta 8.04(\mathrm{~d}, J=8.16 \mathrm{~Hz}, 2 \mathrm{H}), 7.88(\mathrm{~s}, 1 \mathrm{H}), 7.85$ (s, $0.59 \mathrm{H}), 7.74(\mathrm{~d}, J=8.34 \mathrm{~Hz}, 1 \mathrm{H}), 7.50(\mathrm{dd}, J=8.46,1.62 \mathrm{~Hz}, 1 \mathrm{H}), 7.30(\mathrm{~d}, J=8.22$ Hz, 2 H), 3.02-3.00 (m, 2.48 H), 2.57 (s, 3 H), 2.42 (s, 3 H).

${ }^{1} \mathrm{H}-\mathrm{NMR}$ indicates $41 \%$ deuterium incorporation at the 4-position of $3 \mathrm{ba}$, and $17 \%$ deuterium incorporation at the methyl group of $\mathbf{3 b a}$.

\section{Step 2:}
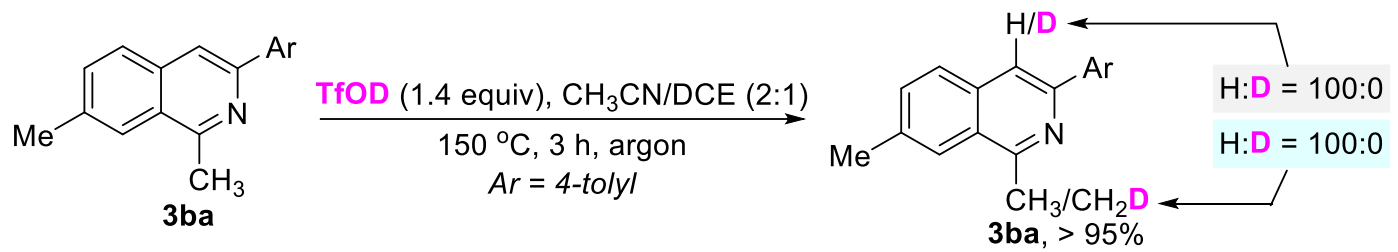

3 ba

An oven-dried Schlenk tube was charged with 3 ba $(0.1 \mathrm{mmol})$, 1,2-dichloroethane $(0.4 \mathrm{~mL})$ and acetonitrile $(0.8 \mathrm{~mL})$ under argon atmosphere. The mixture was then stirred at room temperature, and TfOD $(0.14 \mathrm{mmol})$ was added by a syringe under argon. The reaction mixture was sealed and heated at $150{ }^{\circ} \mathrm{C}$ in an oil bath for 3 hours. Upon completion, the resulting mixture was cooled to room temperature, and triethylamine $(0.5 \mathrm{ml})$ was added by a syringe. The resulting solution was stirred for more than 1 hour under air, filtered through a short silica gel pad and washed with ethyl acetate. The above solution was evaporated under vacuum, and pure $\mathbf{3 b a}$ was recovered in more than $95 \%$.

${ }^{1} H$-NMR indicates no deuterium was incorporated either at the 4-position or the methyl group of $3 \mathbf{b a}$. 


\section{Step 3}

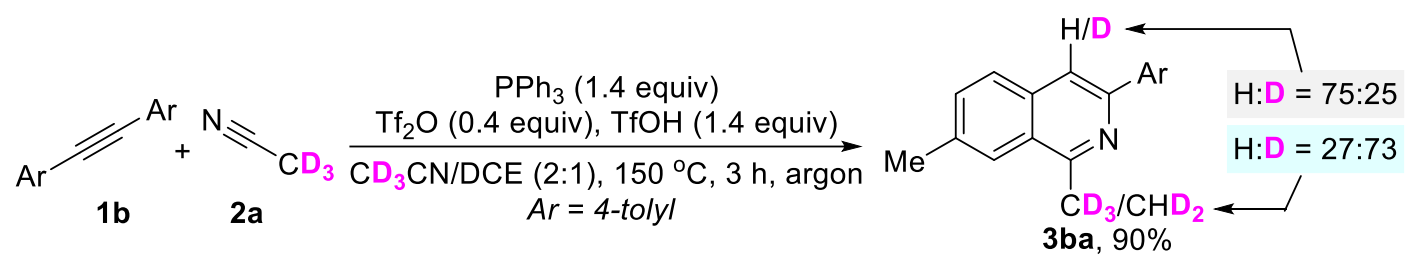

An oven-dried Schlenk tube was charged with 1,2-bis( $p$-tolyl)ethyne 1 (0.1 mmol), triphenylphosphine $(36.7 \mathrm{mg}, 0.14 \mathrm{mmol})$. After the tube was filled with argon, 1,2dichloroethane $(0.4 \mathrm{~mL})$ and $\mathrm{D}_{3}$-acetonitrile $(0.8 \mathrm{~mL})$ were added by a syringe. Then the mixture was stirred at room temperature, and trifluoromethanesulfonic anhydride $(0.04 \mathrm{mmol})$ and $\mathrm{TfOH}(0.14 \mathrm{mmol})$ were added sequentially by a syringe under argon. The reaction mixture was sealed and heated at $150{ }^{\circ} \mathrm{C}$ in an oil bath for 3 hours. Upon completion, the resulting mixture was cooled to room temperature, and triethylamine $(0.5 \mathrm{ml})$ was added by a syringe. The resulting solution was stirred for more than 1 hour under air, filtered through a short silica gel pad and washed with ethyl acetate. The above solution was evaporated under vacuum, and the residue was purified by silica gel column with petroleum ether/acetone as the eluent to give product $\mathbf{3 b a}$.

${ }^{1} \mathbf{H}$ NMR (600 MHz, CDCl $) \delta 8.03(\mathrm{~d}, J=7.86 \mathrm{~Hz}, 2 \mathrm{H}), 7.87(\mathrm{~s}, 1 \mathrm{H}), 7.85(\mathrm{~s}$, $0.75 \mathrm{H}), 7.74(\mathrm{~d}, J=8.22 \mathrm{~Hz}, 1 \mathrm{H}), 7.50(\mathrm{dd}, J=8.43,1.68 \mathrm{~Hz}, 1 \mathrm{H}), 7.30(\mathrm{~d}, J=7.92$ Hz, $2 \mathrm{H}), 3.02-2.98$ (m, 0.81 H), 2.57 (s, $3 \mathrm{H}), 2.42$ (s, $3 \mathrm{H})$.

${ }^{1} \mathrm{H}$-NMR indicates $25 \%$ deuterium incorporation at the 4-position of $3 \mathbf{b a}$, and $27 \%$ hydrogen incorporation at the $C_{3}$ group of $\mathbf{3 b a}$.

\section{Exploring the possibility of the involvement of intermediate VI}

\section{Synthesis $^{[25]}$ :}<smiles>Brc1cncc2ccccc12</smiles>

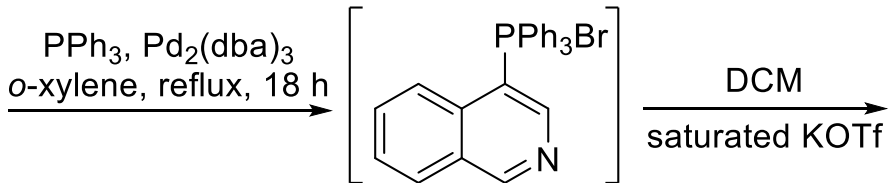<smiles>Pc1cncc2ccccc12</smiles>

$14,5 \%$ yield

4-Bromoisoquinoline (395.3 mg, $1.899 \mathrm{mmol}), \mathrm{PPh}_{3}(498.1 \mathrm{mg}, 1.89 \mathrm{mmol}$ ), and $\mathrm{Pd}_{2}(\mathrm{dba})_{3}(18.3 \mathrm{mg}, 0.02 \mathrm{mmol})$ were suspended in $o$-xylene $(1 \mathrm{~mL})$ in an oven-dried $10 \mathrm{~mL}$ Schlenk tube and heated to reflux under argon for $18 \mathrm{~h}$ in an oil bath. The reaction 
mixture was cooled to room temperature and washed with ethyl acetate and diethyl ether. The resulting solid was dissolved in DCM $(1 \mathrm{~mL})$ and stirred with $60 \mathrm{~mL}$ of saturated aqueous solution of KOTf for $1 \mathrm{~h}$. Then the mixture was extracted with DCM $(20 \mathrm{~mL} \times 3)$. The organic layer was dried over anhydrous $\mathrm{Na}_{2} \mathrm{SO}_{4}$, concentrated under vacuum to give slightly grey solid $\mathbf{1 4}$ in $5 \%$ yield $(51.1 \mathrm{mg})$. The spectroscopic data for 14 are as follows:

${ }^{1}$ H NMR (600 MHz, CDCl $) \delta 9.73(\mathrm{~s}, 1 \mathrm{H}), 8.39(\mathrm{~s}, 1 \mathrm{H}), 8.35(\mathrm{~d}, J=8.82 \mathrm{~Hz}$, $1 \mathrm{H}), 7.89(\mathrm{t}, J=8.22 \mathrm{~Hz}, 3 \mathrm{H}), 7.86(\mathrm{t}, J=7.68 \mathrm{~Hz}, 1 \mathrm{H}), 7.76-7.74(\mathrm{~m}, 6 \mathrm{H}), 7.72-$ 7.67 (m, $7 \mathrm{H}), 7.37$ (d, $J=8.46 \mathrm{~Hz}, 1 \mathrm{H})$.

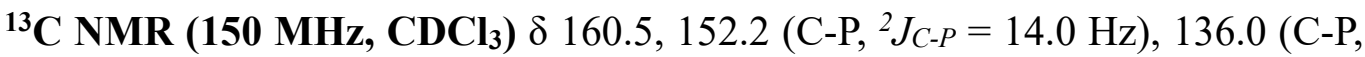
$\left.{ }^{4} J_{C-P}=3.2 \mathrm{~Hz}\right), 135.0\left(\mathrm{C}-\mathrm{P},{ }^{3} J_{C-P}=6.8 \mathrm{~Hz}\right), 134.3\left(\mathrm{C}-\mathrm{P},{ }^{3} J_{C-P}=10.8 \mathrm{~Hz}\right), 134.2,131.1$ $\left(\mathrm{C}-\mathrm{P},{ }^{2} J_{\mathrm{C}-\mathrm{P}}=13.2 \mathrm{~Hz}\right), 130.9\left(\mathrm{C}-\mathrm{P},{ }^{1} J_{\mathrm{C}-\mathrm{P}}=75.9 \mathrm{~Hz}\right), 130.7,130.1,125.1\left(\mathrm{C}-\mathrm{P}, J_{C-P}=5.8\right.$ $\mathrm{Hz}), 120.8\left(\mathrm{C}-\mathrm{F},{ }^{1} J_{C-F}=319.6 \mathrm{~Hz}\right), 116.9\left(\mathrm{C}-\mathrm{P},{ }^{1} J_{C-P}=88.8 \mathrm{~Hz}\right)$.

${ }^{31}$ P NMR (161.9 MHz, $\left.\mathbf{C D C l}_{3}\right) 19.89$.

HR-ESI-MS: [M-OTf] ${ }^{+} \mathrm{m} / \mathrm{z}$ calcd for $\mathrm{C}_{27} \mathrm{H}_{21} \mathrm{NP}, 390.1406$; found 390.1403 .

\section{Treating 14 with TfOH:}<smiles>Pc1cncc2ccccc12</smiles>

14

\section{TfOH (1.4 equiv), MeCN/DCE (2:1), $150^{\circ} \mathrm{C}, 3 \mathrm{~h}$, argon}<smiles>c1ccc2cnccc2c1</smiles>

$>90 \%$

An oven-dried 10-mL Schlenk tube was charged with 14 (108.0 mg, $0.20 \mathrm{mmol})$. After the tube was filled with argon, 1,2-dichloroethane $(0.8 \mathrm{~mL})$ and acetonitrile (1.6 $\mathrm{mL}$ ) were added by a syringe. Then the mixture was stirred at room temperature, and $\mathrm{TfOH}(0.28 \mathrm{mmol}, 1.4$ equiv) was added by a syringe under argon. The reaction mixture was sealed and heated at $150{ }^{\circ} \mathrm{C}$ in an oil bath for 3 hours. Upon completion, the resulting mixture was cooled to room temperature, and triethylamine $(1.0 \mathrm{ml})$ was added by a syringe. The resulting solution was stirred for more than 1 hour under air, filtered through a short silica gel pad and washed with ethyl acetate. The above solution was evaporated under vacuum, and the residue was purified by silica gel column with 
petroleum ether/acetone as the eluent to give pure product isoquinoline in about $24 \mathrm{mg}$ (ca. 90\% isolated yield).

\section{D: HR-ESI-MS of mixture after the reaction was conducted under standard conditions:}
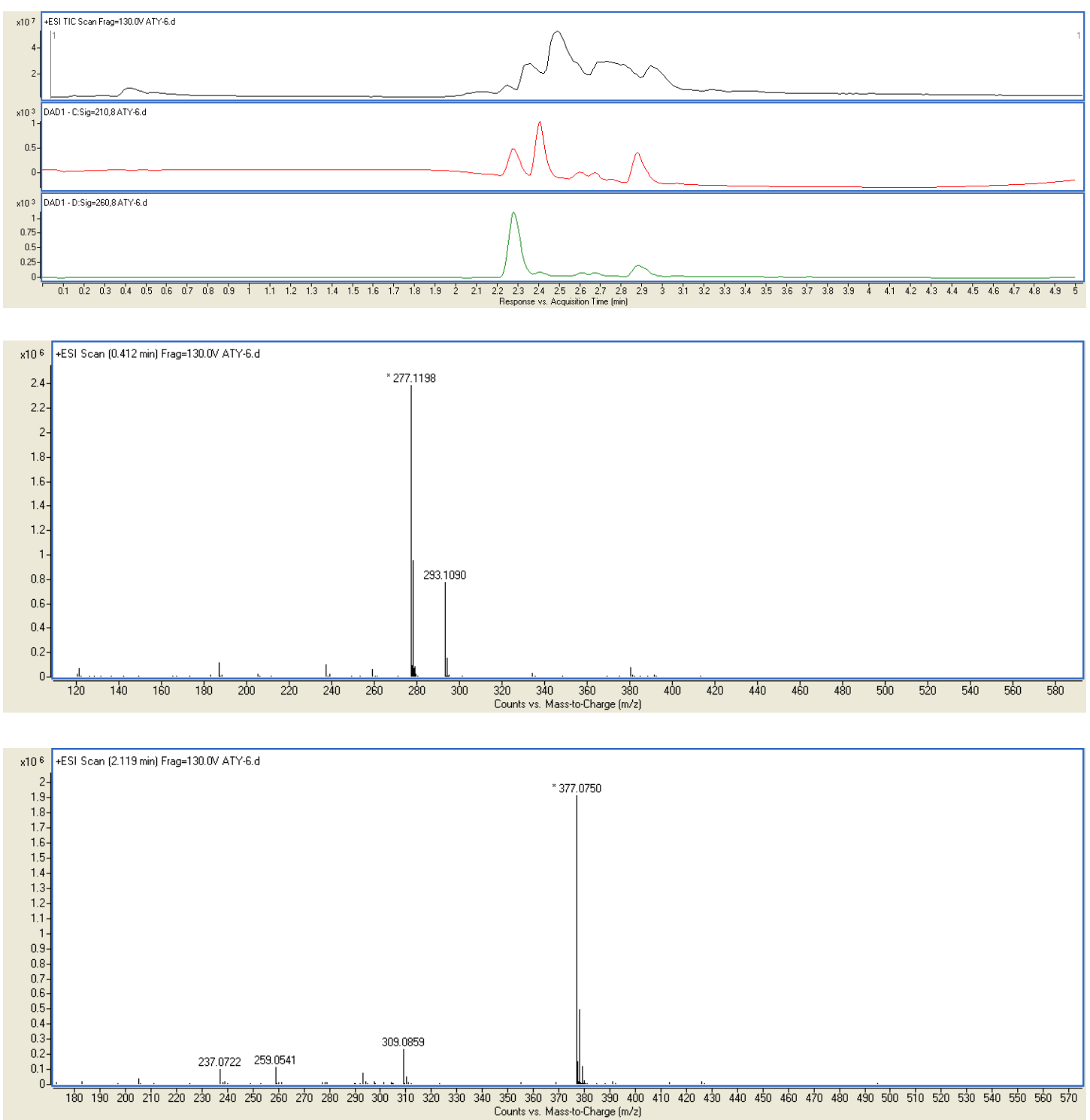

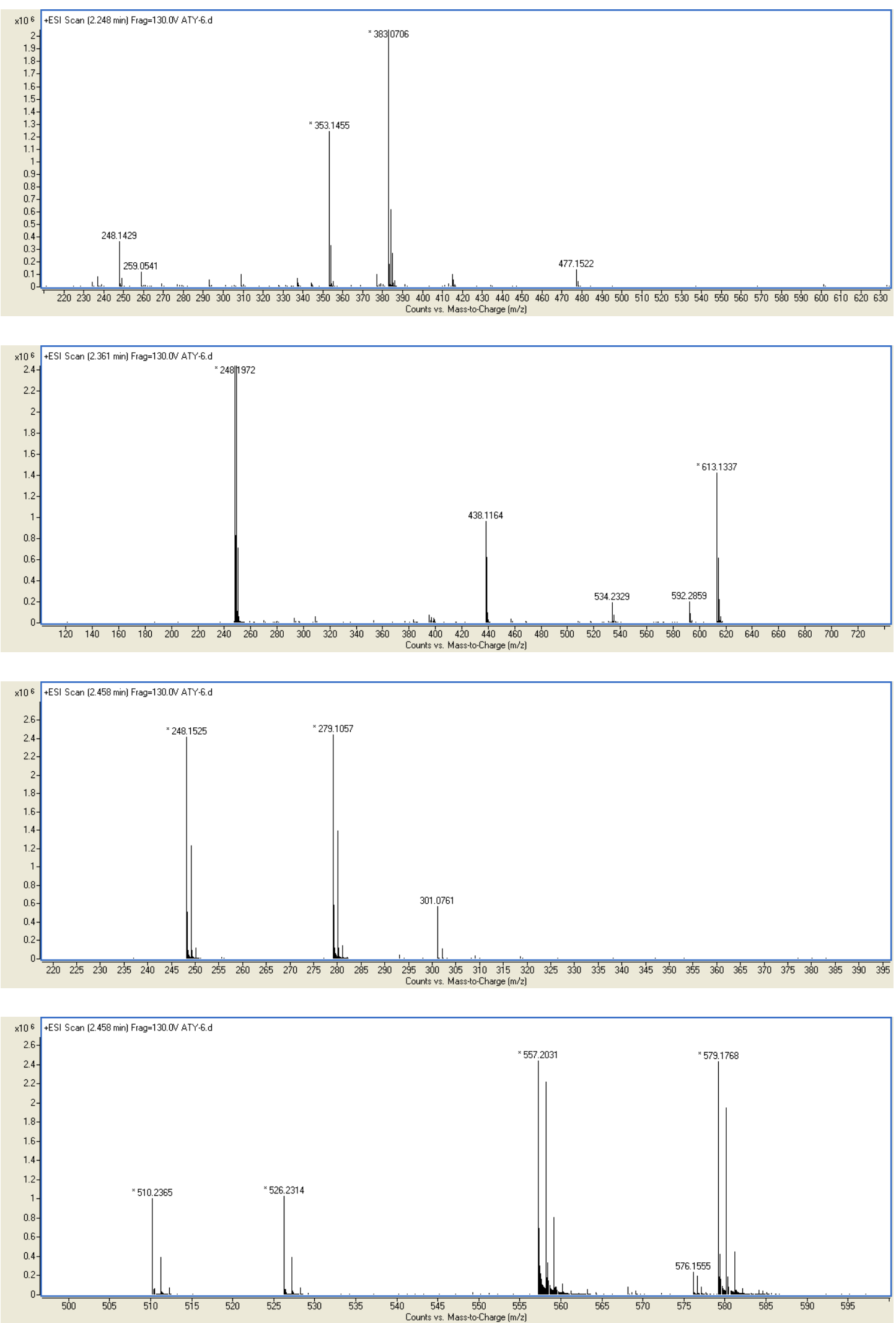

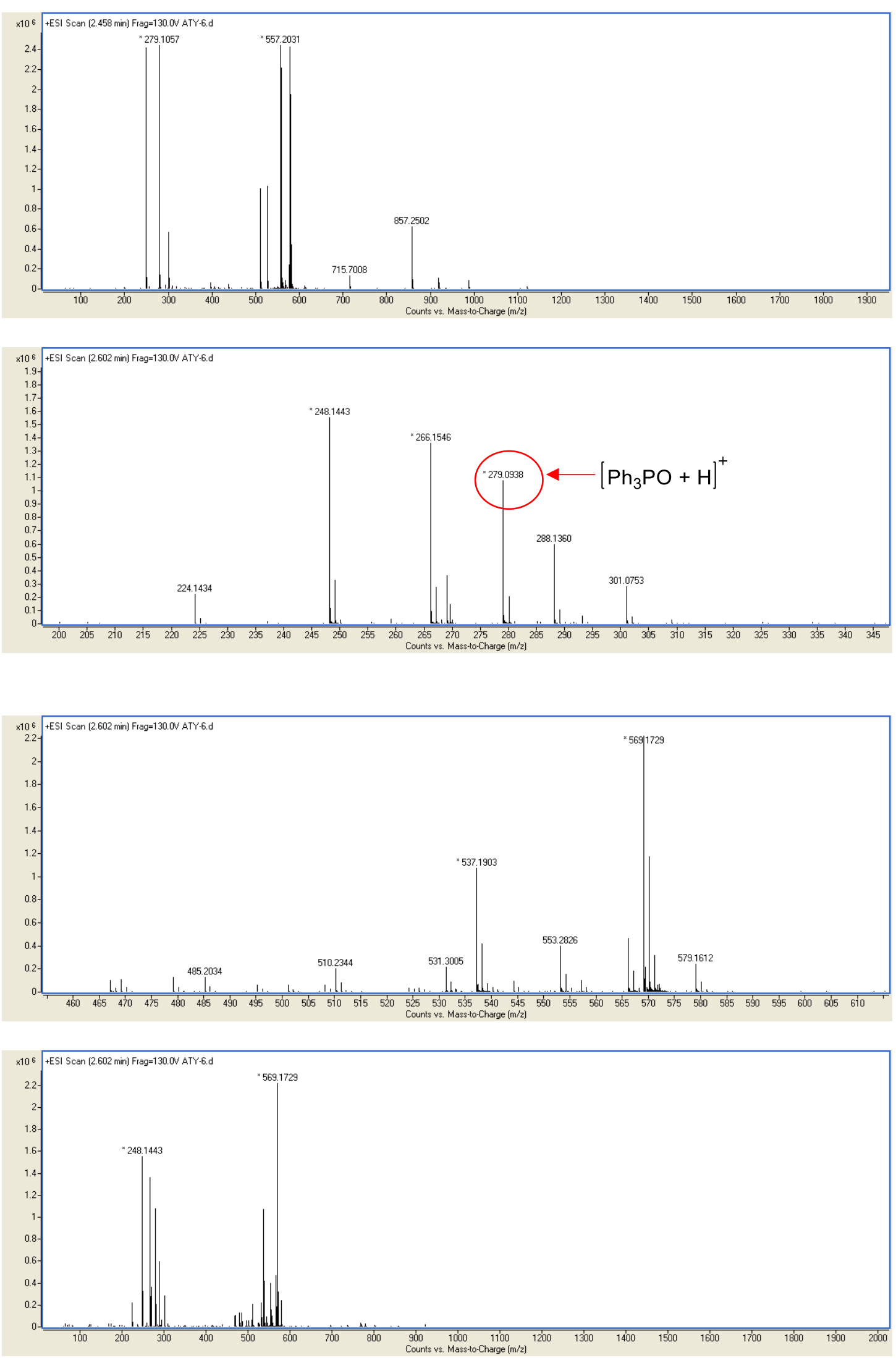

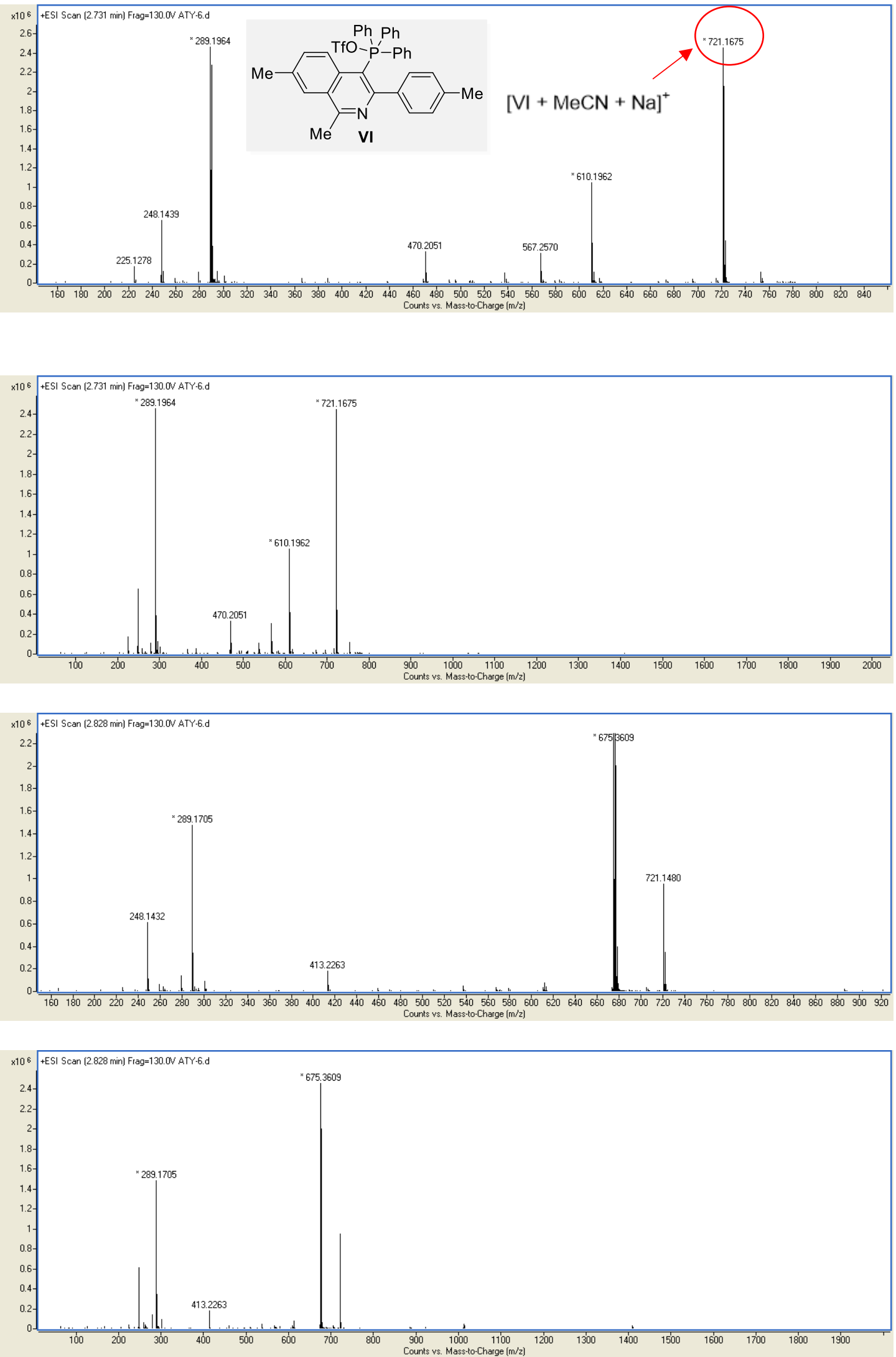

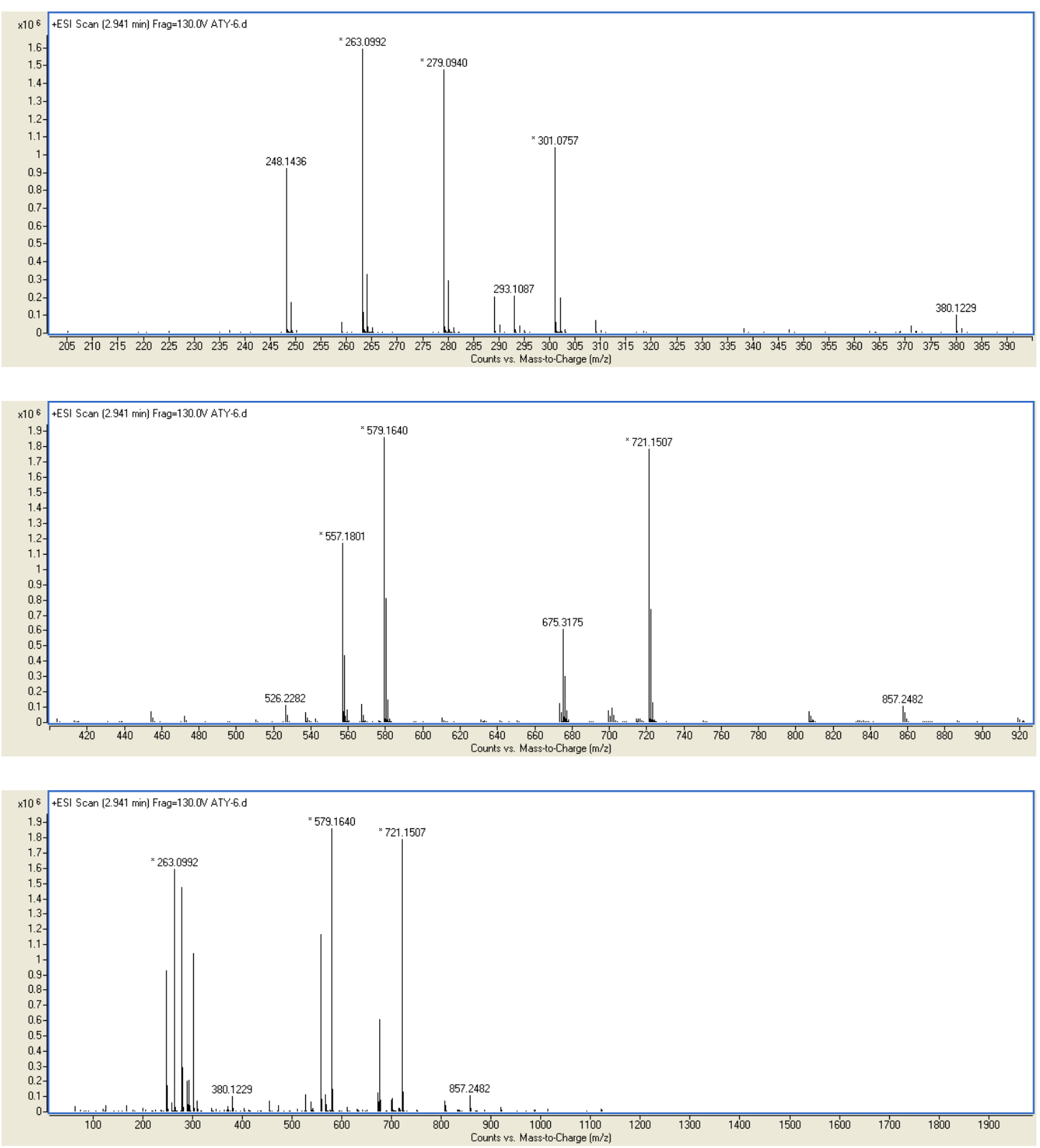

Figure S2. HR-ESI-MS spectrums for the reaction mixture of $1 \mathrm{~b}$ under standard conditions.

These findings suggest that the phosphorane-type (or phosphonium) compounds most probably serve as a key intermediate to release the final products through an irreversible protonation reaction.

\section{References}

[1] Y. Unoh, K. Hirano, M. Miura, J. Am. Chem. Soc. 2017, 139, 6106.

[2] T. N. Gieshoff, A. Welther, M. T. Kessler, M. H. G. Prechtl, A. J. von Wangelin, 
Chem. Commun. 2014, 50, 2261.

[3] Á. M. Martínez, J. Echavarren, I. Alonso, N. Rodríguez, R. G. Arrayás, J. C. Carretero, Chem. Sci. 2015, 6, 5802.

[4] B. Zhou, Z. Wu, W. Qi, X. Sun, Y. Zhang, Adv. Synth. Catal. 2018, 360, 4480.

[5] Z.-Y. Tian, S.-M. Wang, S.-J. Jia, H.-X. Song, C.-P. Zhang, Org. Lett. 2017, 19, 5454.

[6] L. Liang, H.-Y. Nium R.-L. Li, Y.-F. Wang, J.-K. Yan, C.-G. Li, H.-M. Guo, Org. Lett. 2020, 22, 6842 .

[7] V. P. Charpe, A. Sagadevan, K. C. Hwang, Green Chem. 2020, 22, 4426.

[8] M. S. Maji, S. Murarka, A. Studer, Org. Lett. 2010, 12, 3878.

[9] L.-W. Qian, M. Sun, J. Dong, Q. Xu, Y. Zhou, S.-F. Yin, J. Org. Chem. 2017, 82, 6764.

[10] S. Zhou, M. Wang, L. Wang, K. Chen, J. Wang, C. Song, J. Zhu, Org. Lett. 2016, $18,5632$.

[11] A. N. Kim, A. Ngamnithiporn, E. R. Welin, M. T. Daiger, C. U. Grünanger, M. D. Bartberger, S. C. Virgil, B. M. Stoltz, ACS Catal. 2020, 10, 3241.

[12] B. Sun, T. Yoshino, M. Kanai, S. Matsunaga, Angew. Chem. Int. Ed. 2015, 54, 12958.

[13] M. Meanwell, B. S. Adluri, Z. Yuan, J. Newton, P. Prevost, M. B. Nodwell, C. M. Friesen, P. Schaffer, R. E. Martin, R. Britton, Chem. Sci. 2018, 9, 5608.

[14] X. Chen, S. Martini, V. Michelet, Adv. Synth. Catal. 2019, 361, 3612.

[15] D. Shabashov, O. Daugulis, Org. Lett. 2005, 7, 3657.

[16] J. Wu, X. Cui, L. Chen, G. Jiang, Y. Wu, J. Am. Chem. Soc. 2009, 131, 13888.

[17] H.-T. Zeng, J.-M. Huang, Org. Lett. 2015, 17, 4276.

[18] Z. Shu, Y. Ye, Y. Deng, Y. Zhang, J. Wang, Angew. Chem. Int. Ed. 2013, 52, 10573.

[19] H.-S. Yeom, S. Kim, S. Shin, Synlett 2008, 924.

[20] J. M. Penney, Tetrahedron Lett. 2004, 45, 2667.

[21] L. Qi, K. Hu, S. Yu, J. Zhu, T. Cheng, X. Wang, J. Chen, H. Wu, Org. Lett. 2017, $19,218$.

[22] Y. Kondo, R. Watanabe, T. Sakamoto, H. Yamanaka, Chem. Pharm. Bull. 1989, 
$37,2814$.

[23] Z. Moussa, S. A. Ahmed, A. S. ElDouhaibi, S. Y. Al-Raqa, Tetrahedron Lett. 2010, $51,1826$.

[24] L. L. Tolstikova, A. V. Bel'skikh, B. A. Shainyan, Russ. J. Gen. Chem. 2011, 81, 474.

[25] A. G. Barrado, J. M. Bayne, T. C. Johnstone, C. W. Lehmann, D. W. Stephan, M. Alcarazo, Dalton Trans. 2017, 46, 16216. 
17. Copies of the ${ }^{1} \mathrm{H}$ NMR for compounds 1

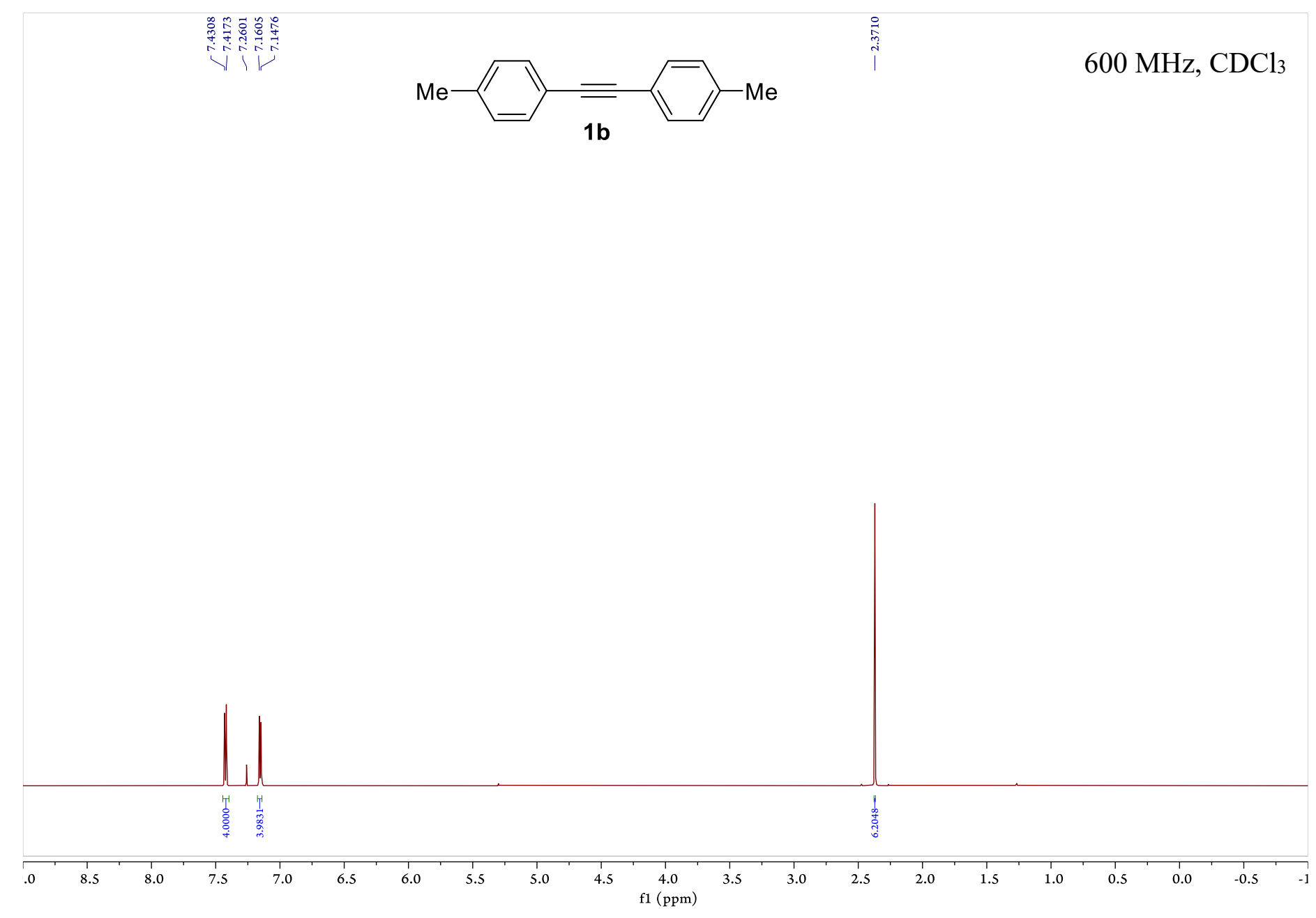




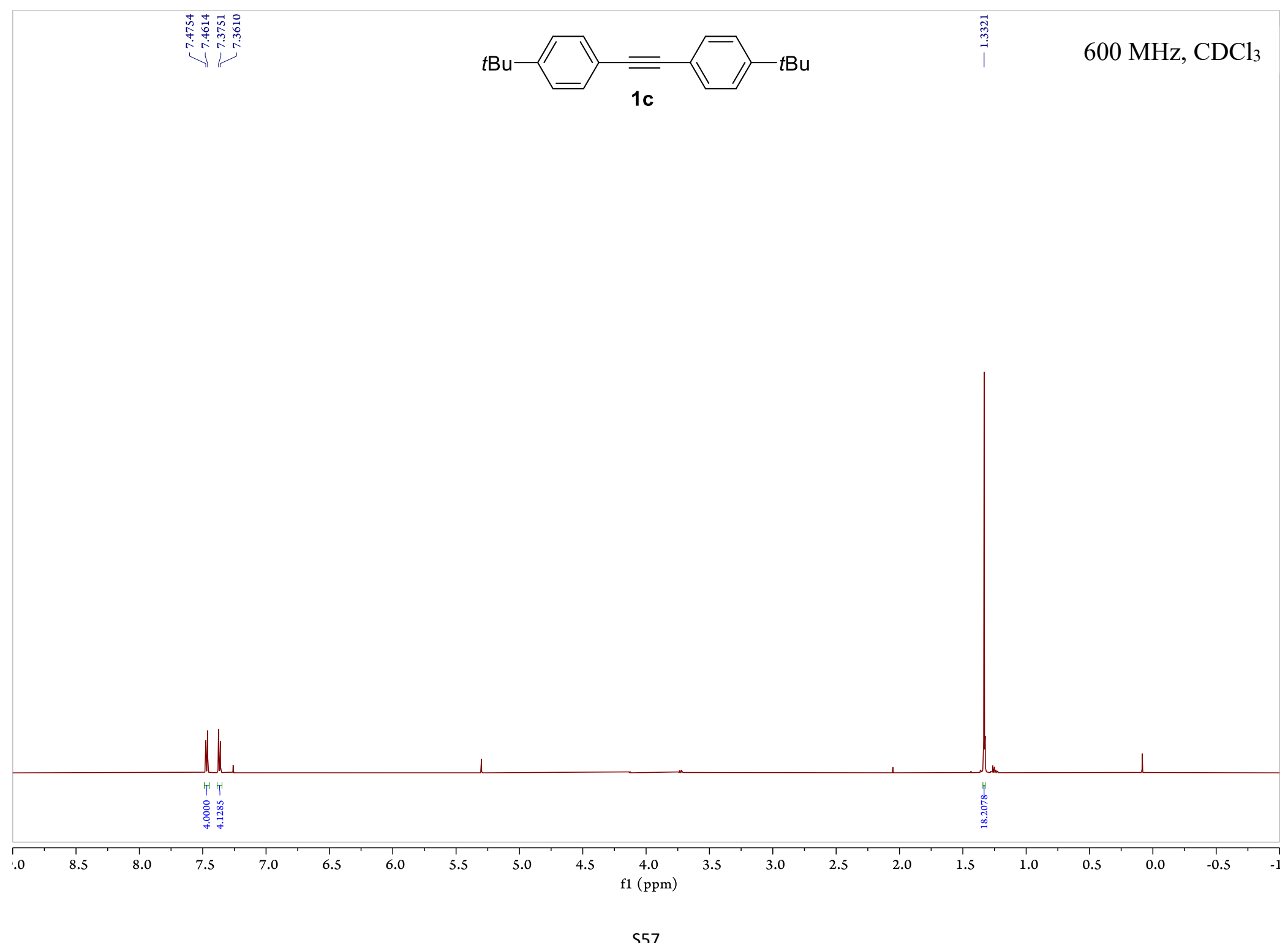




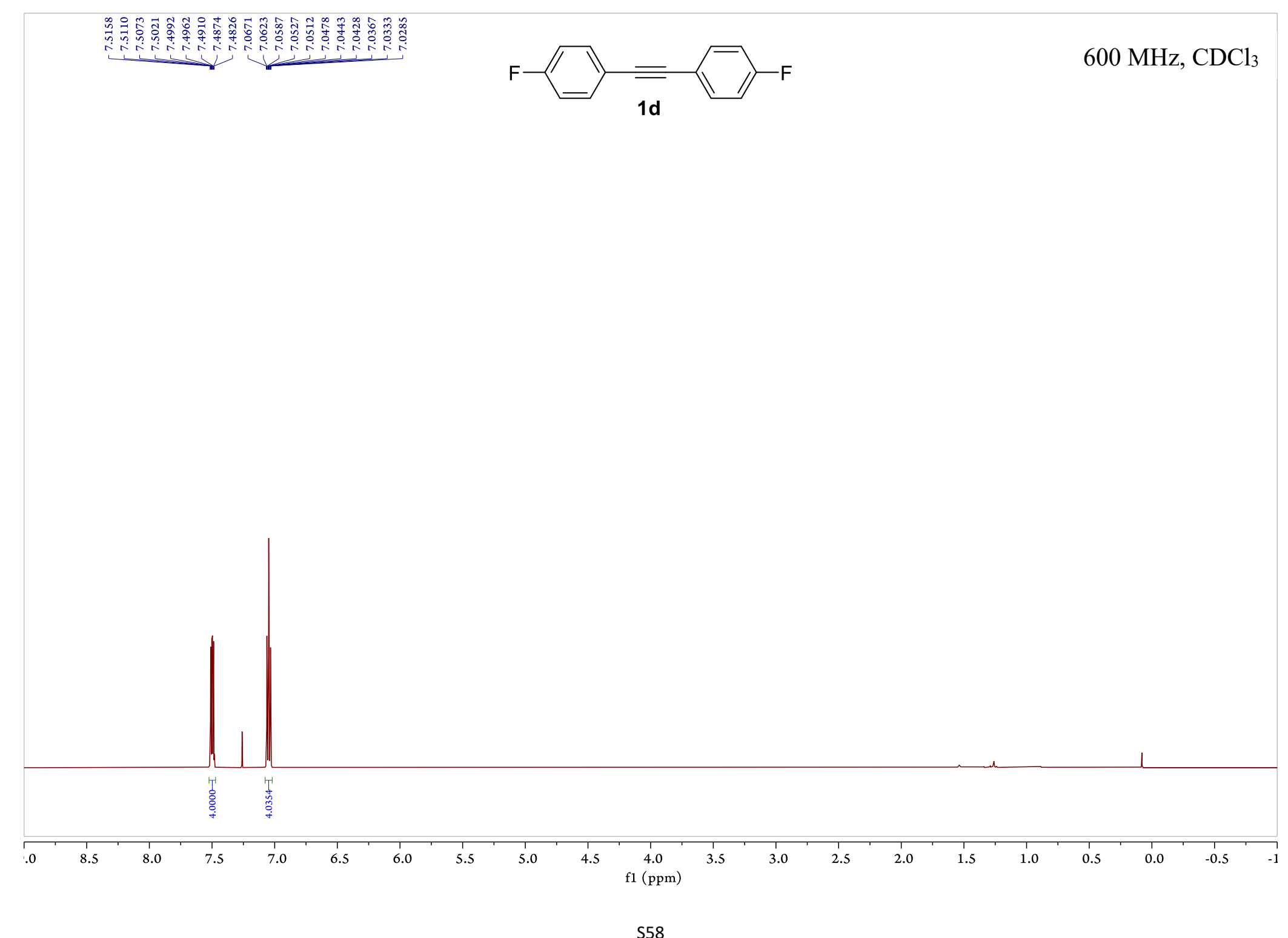




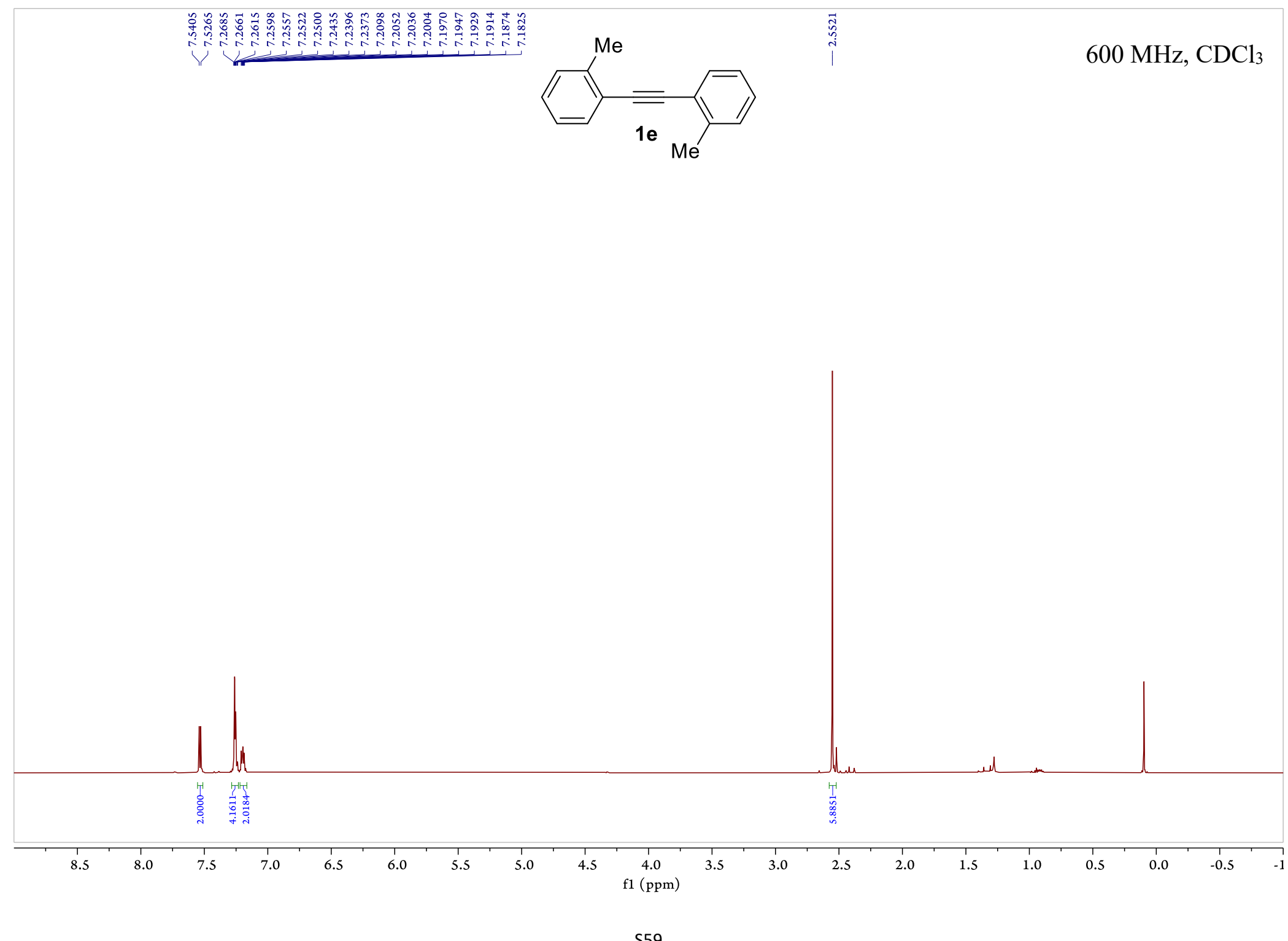




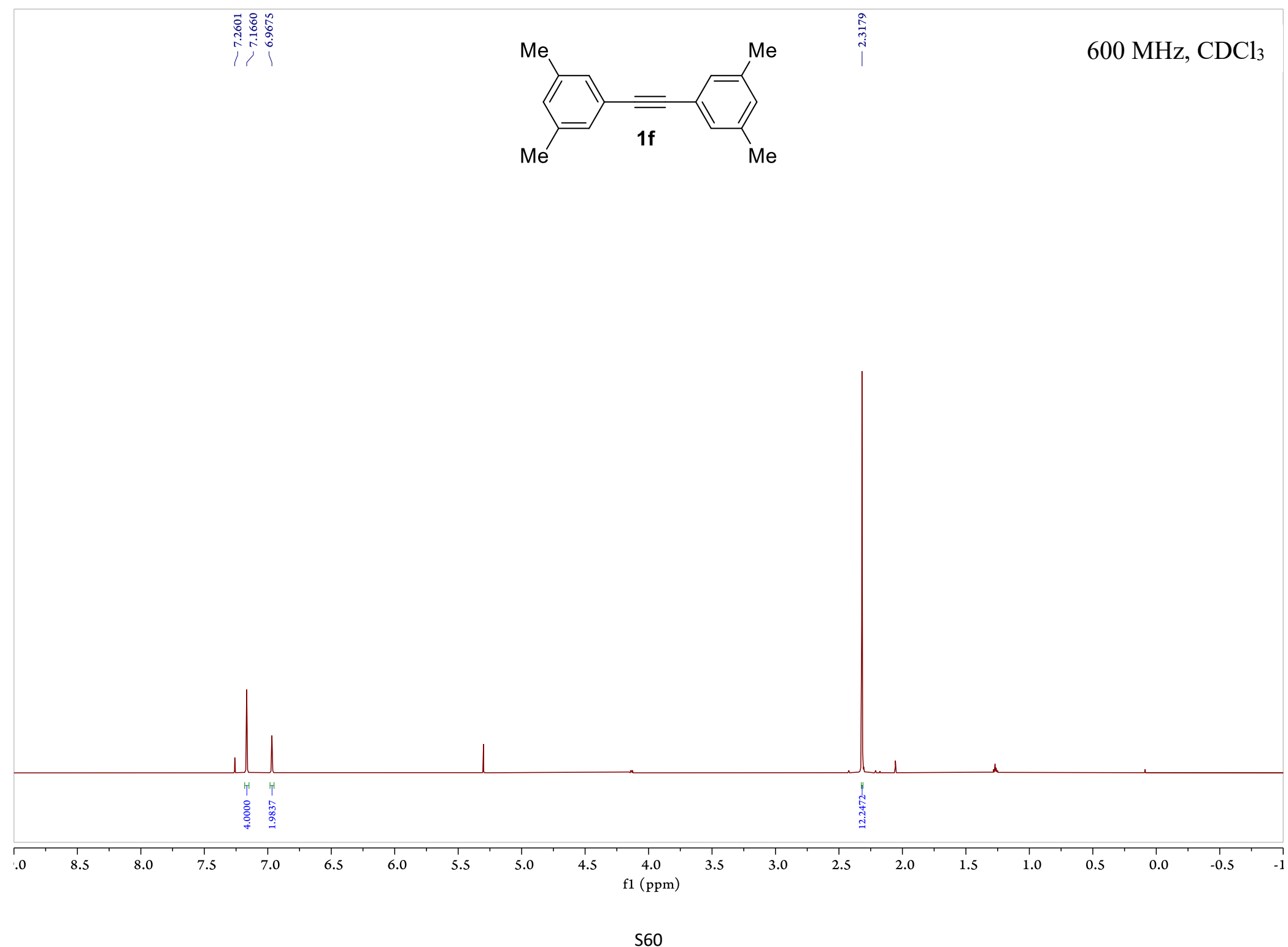




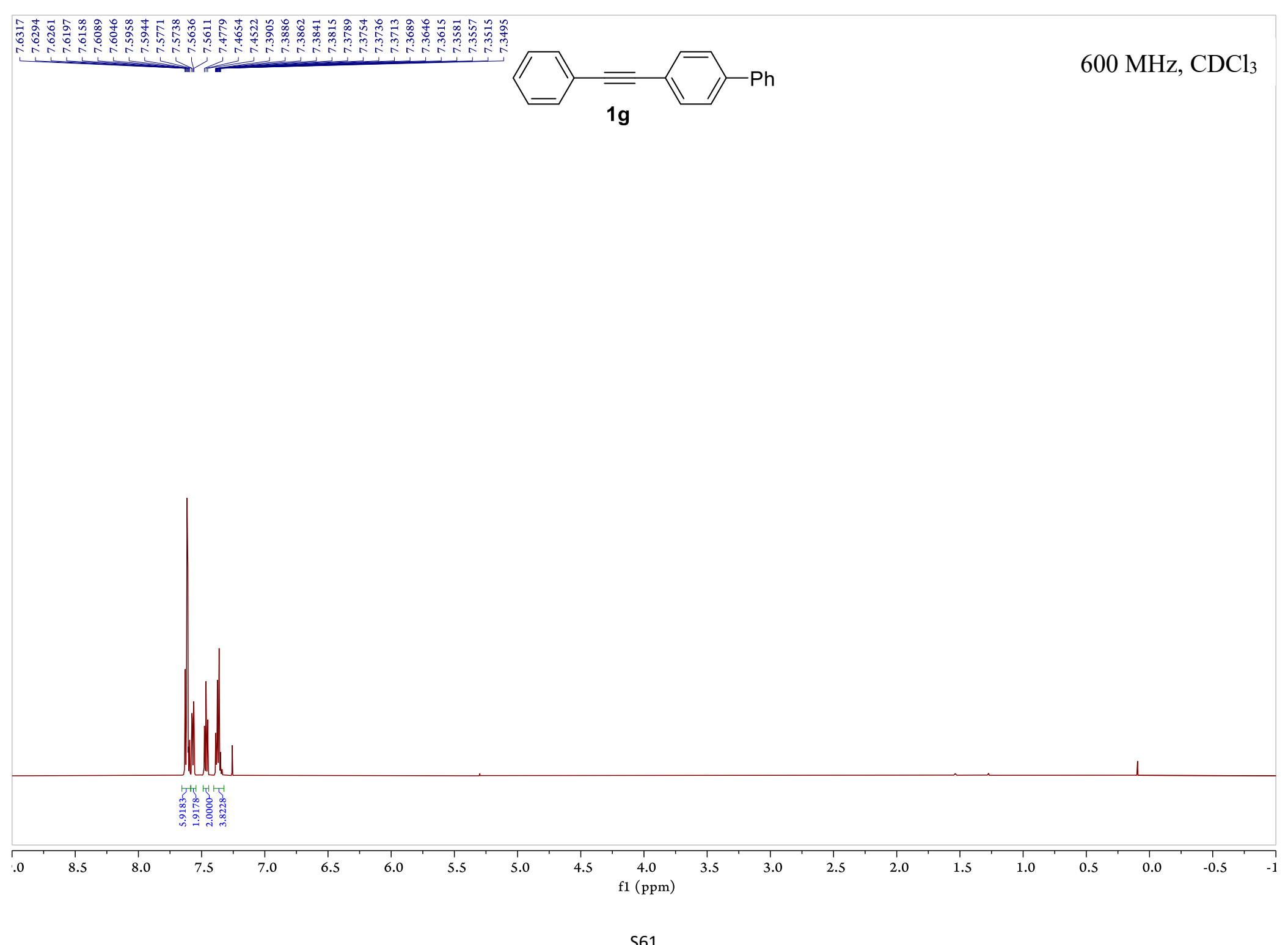

S61 


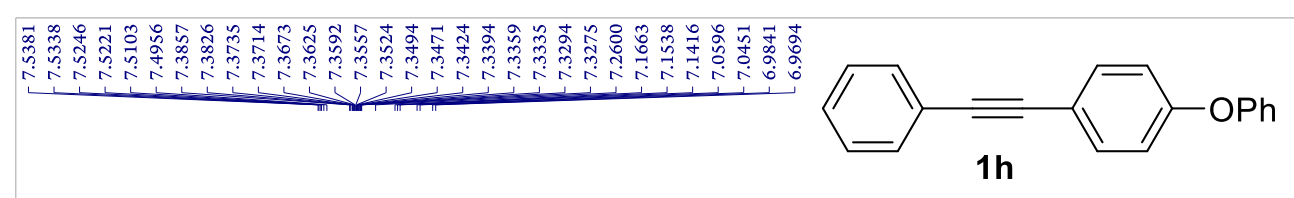

$600 \mathrm{MHz}, \mathrm{CDCl}_{3}$

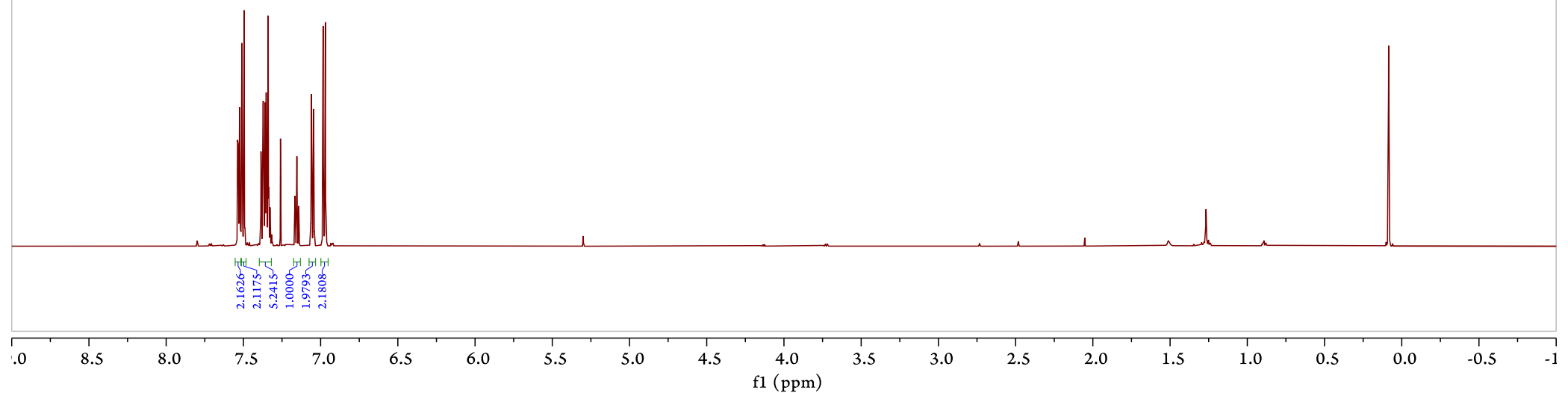

S62 


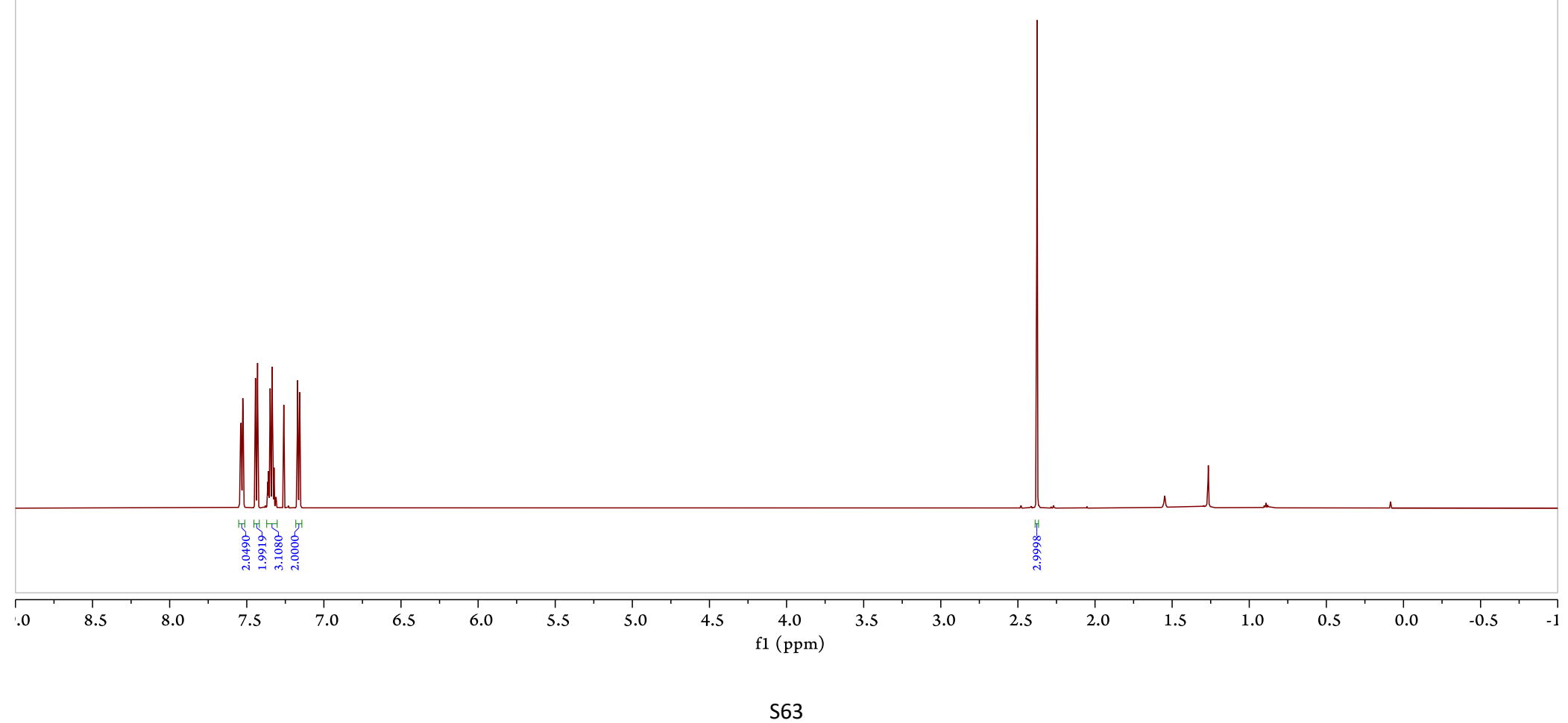




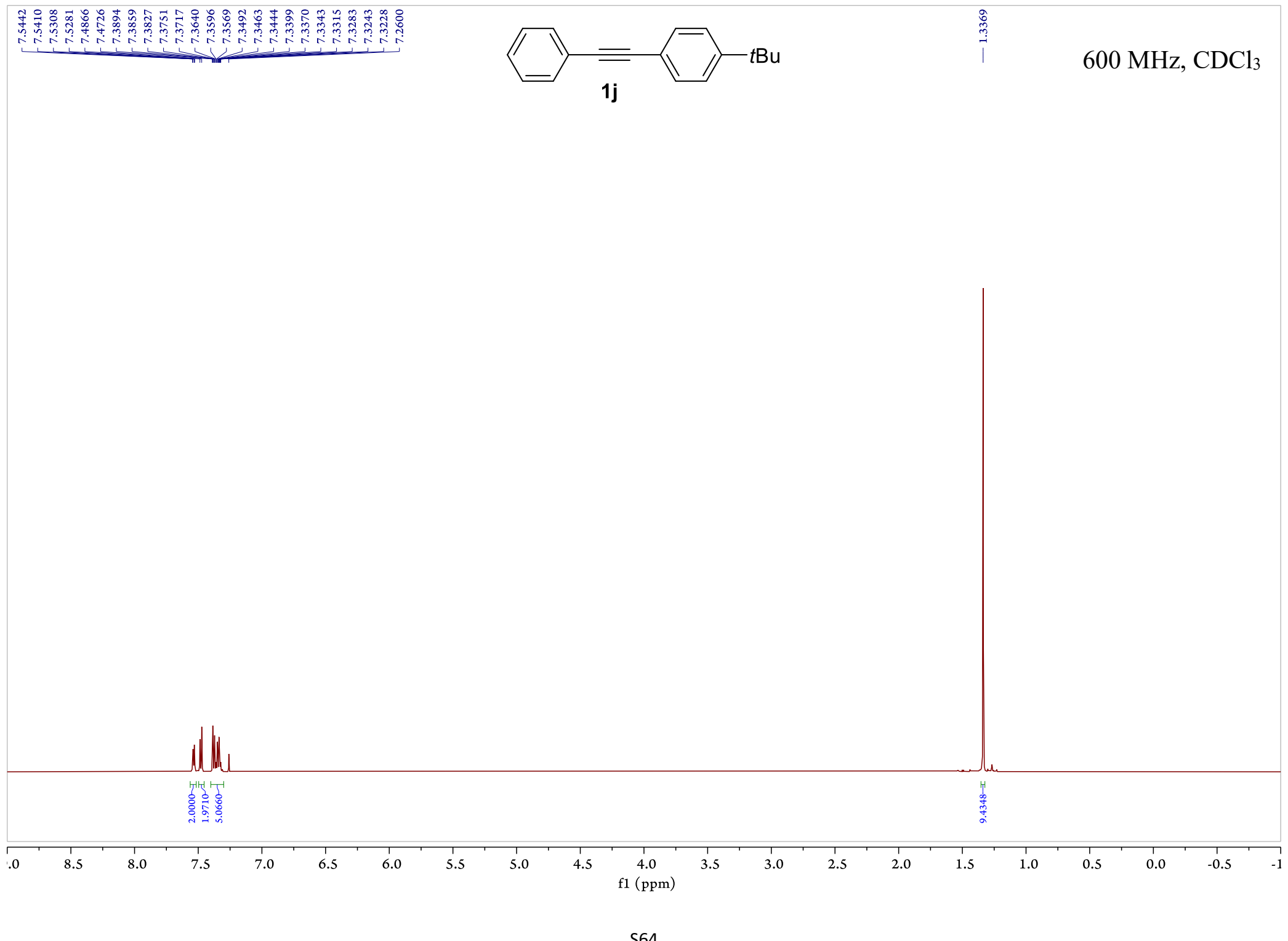




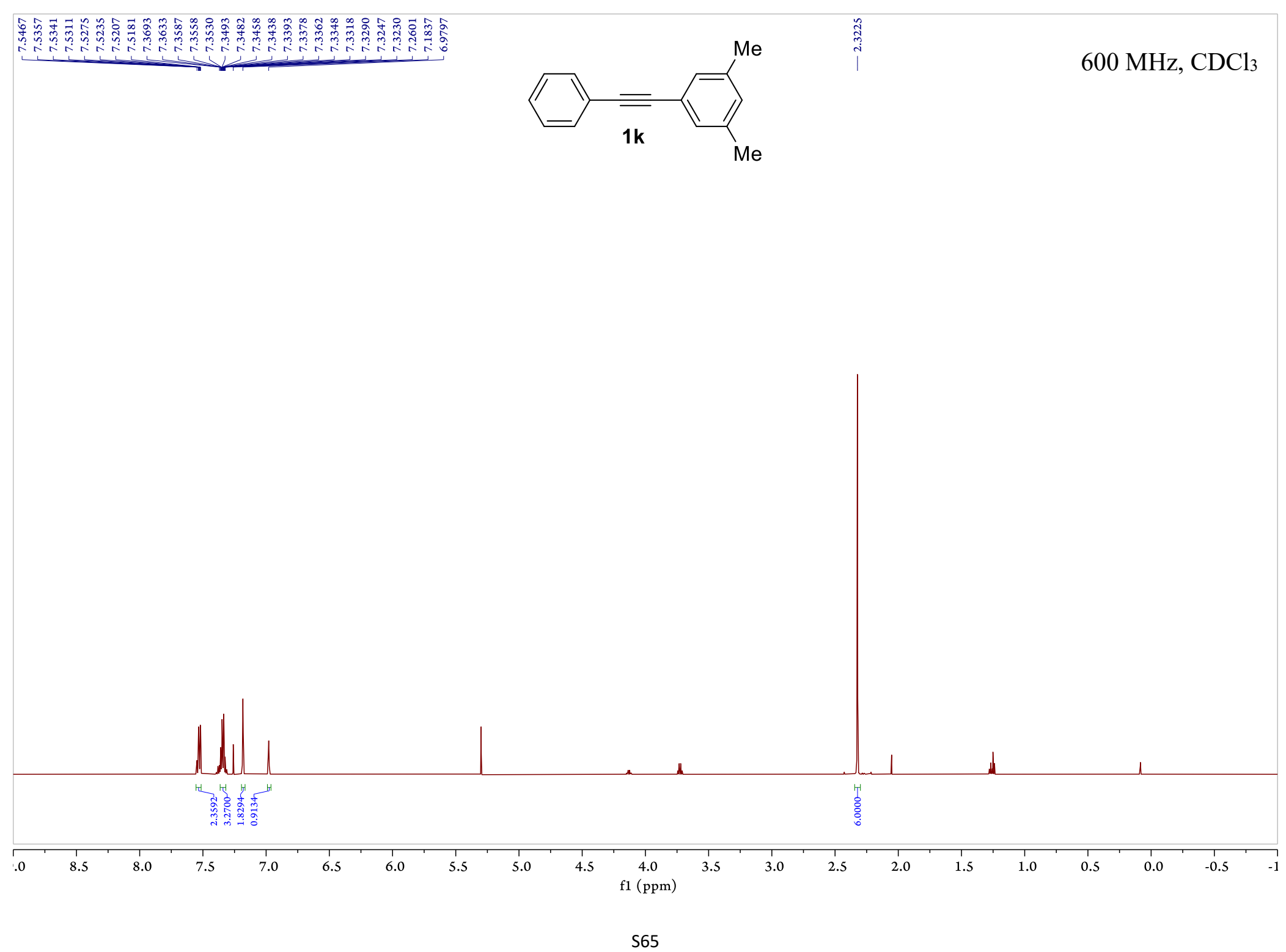




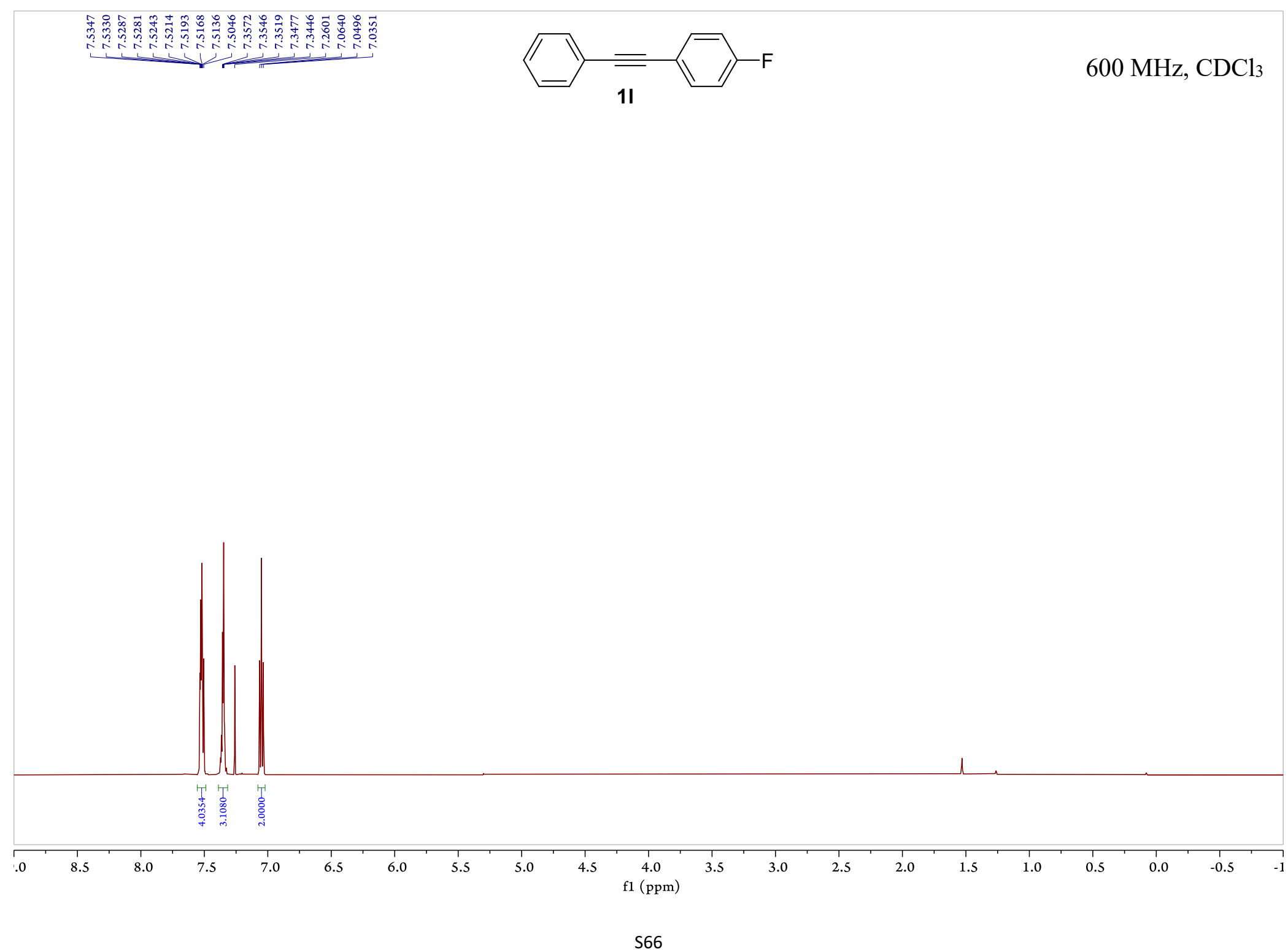




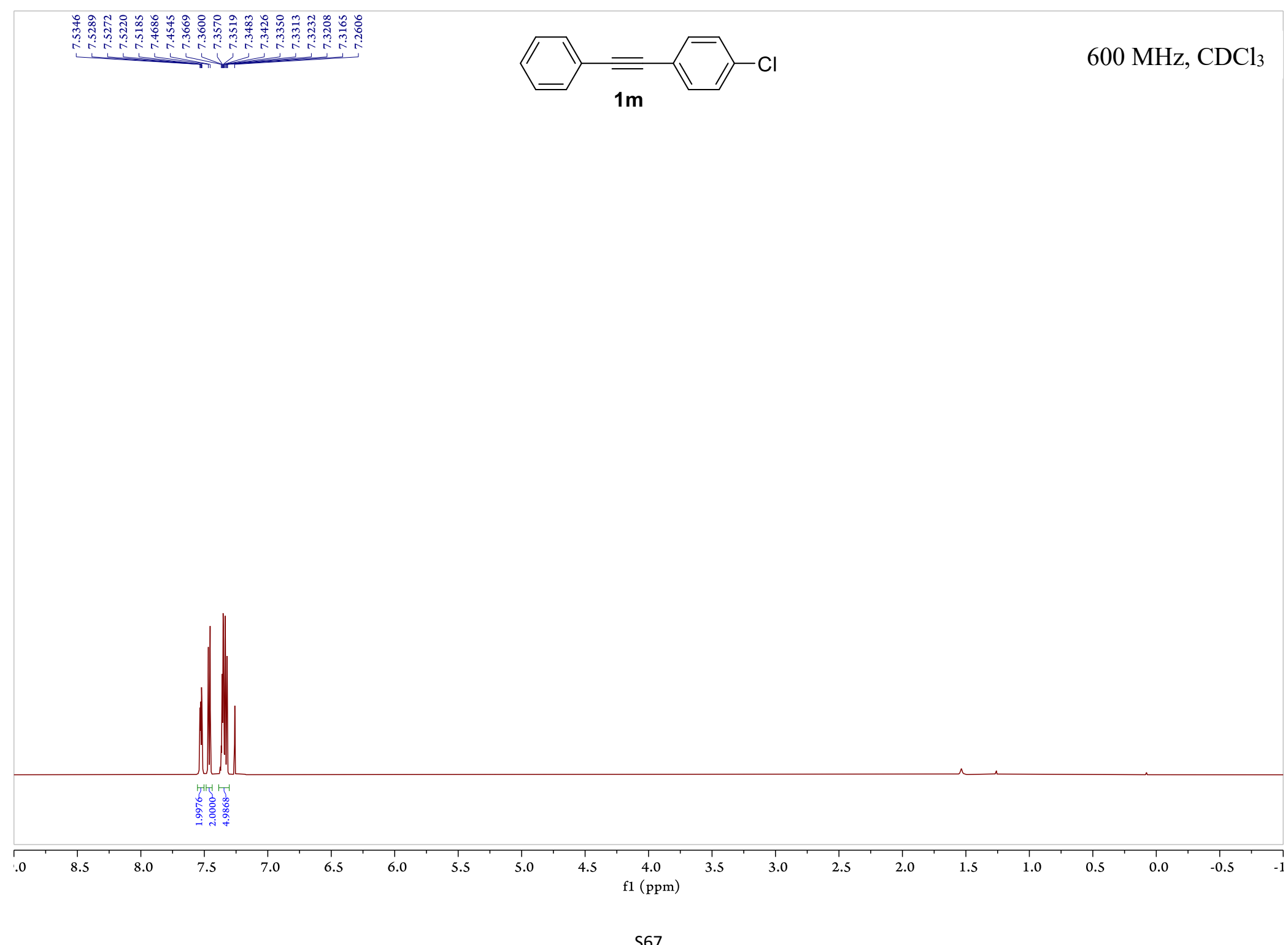




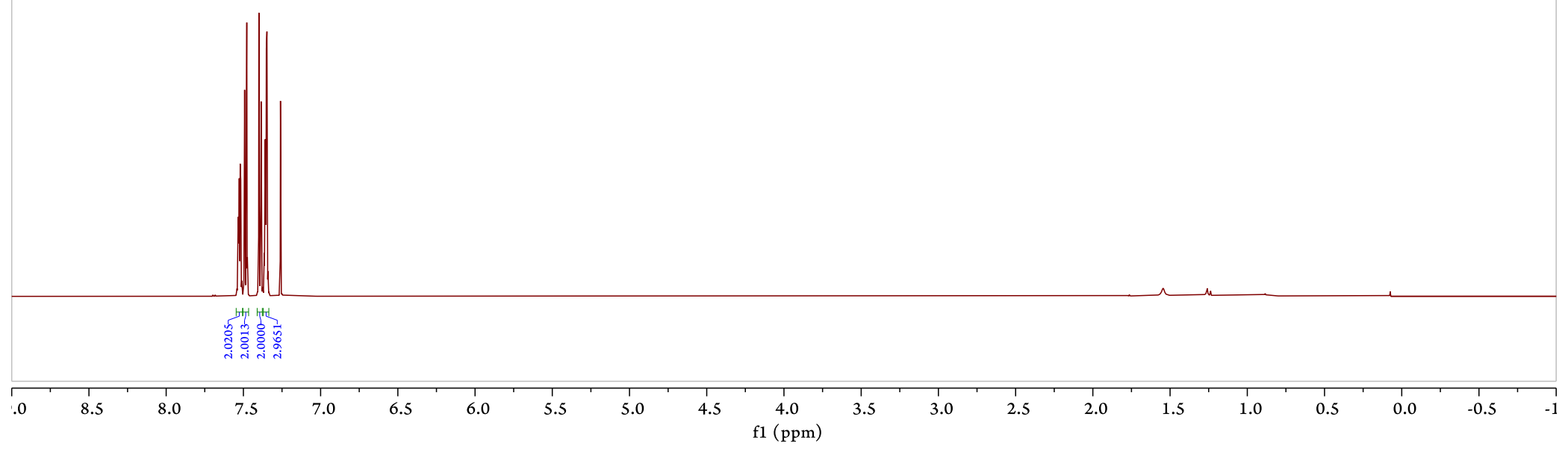




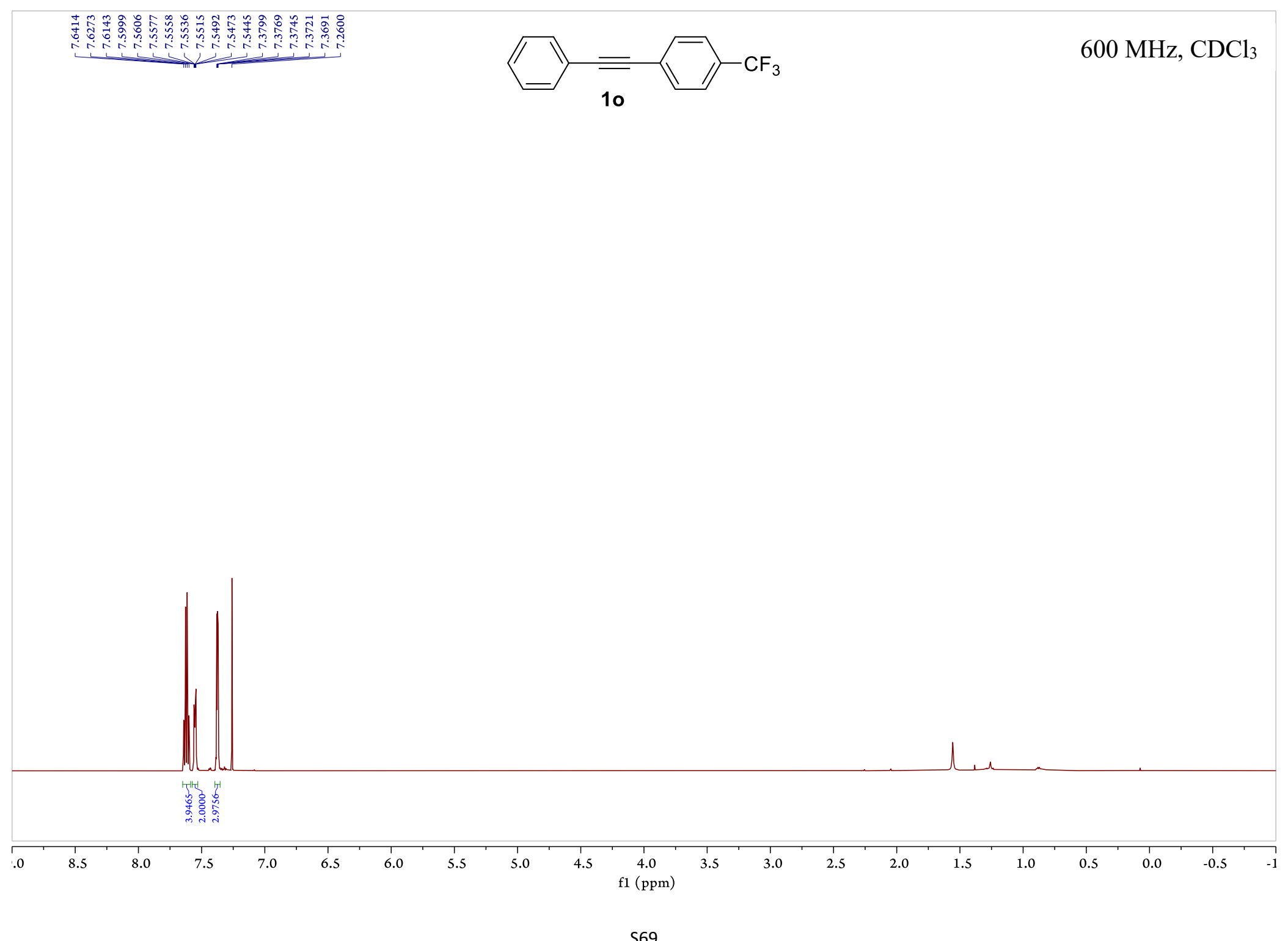




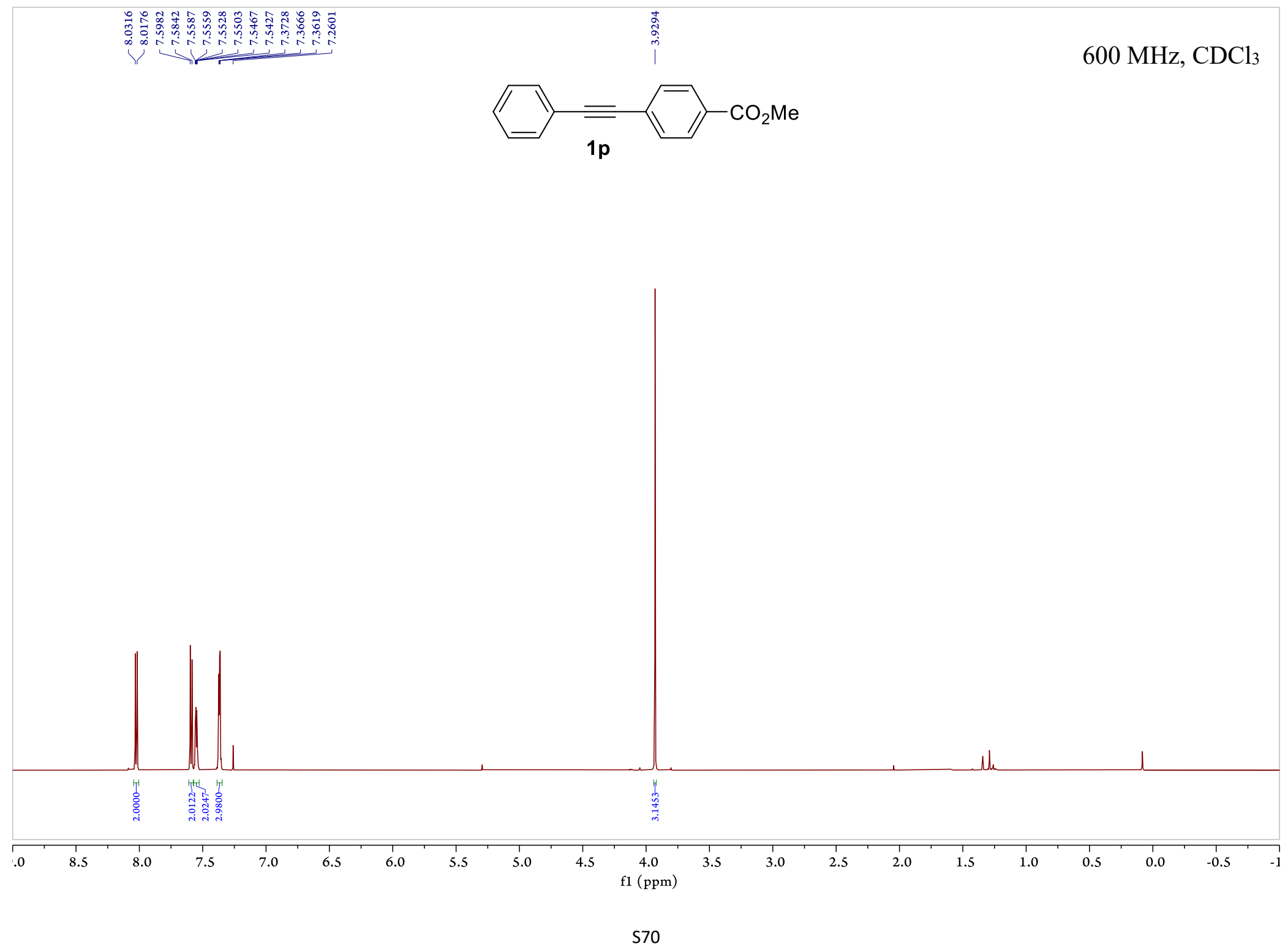




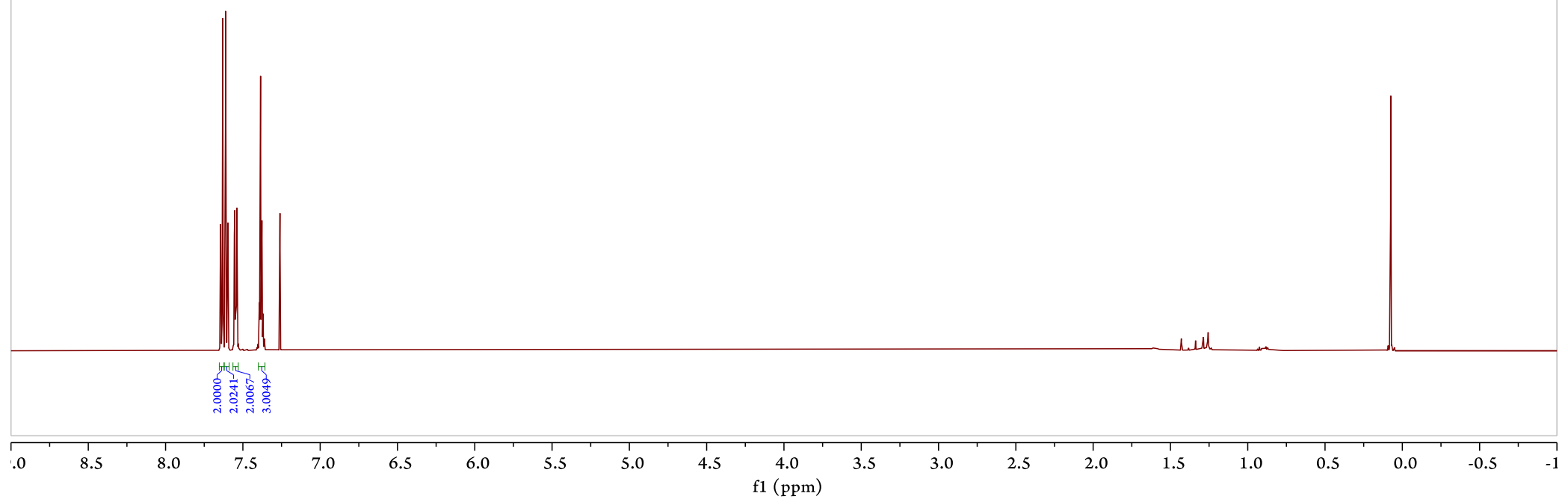




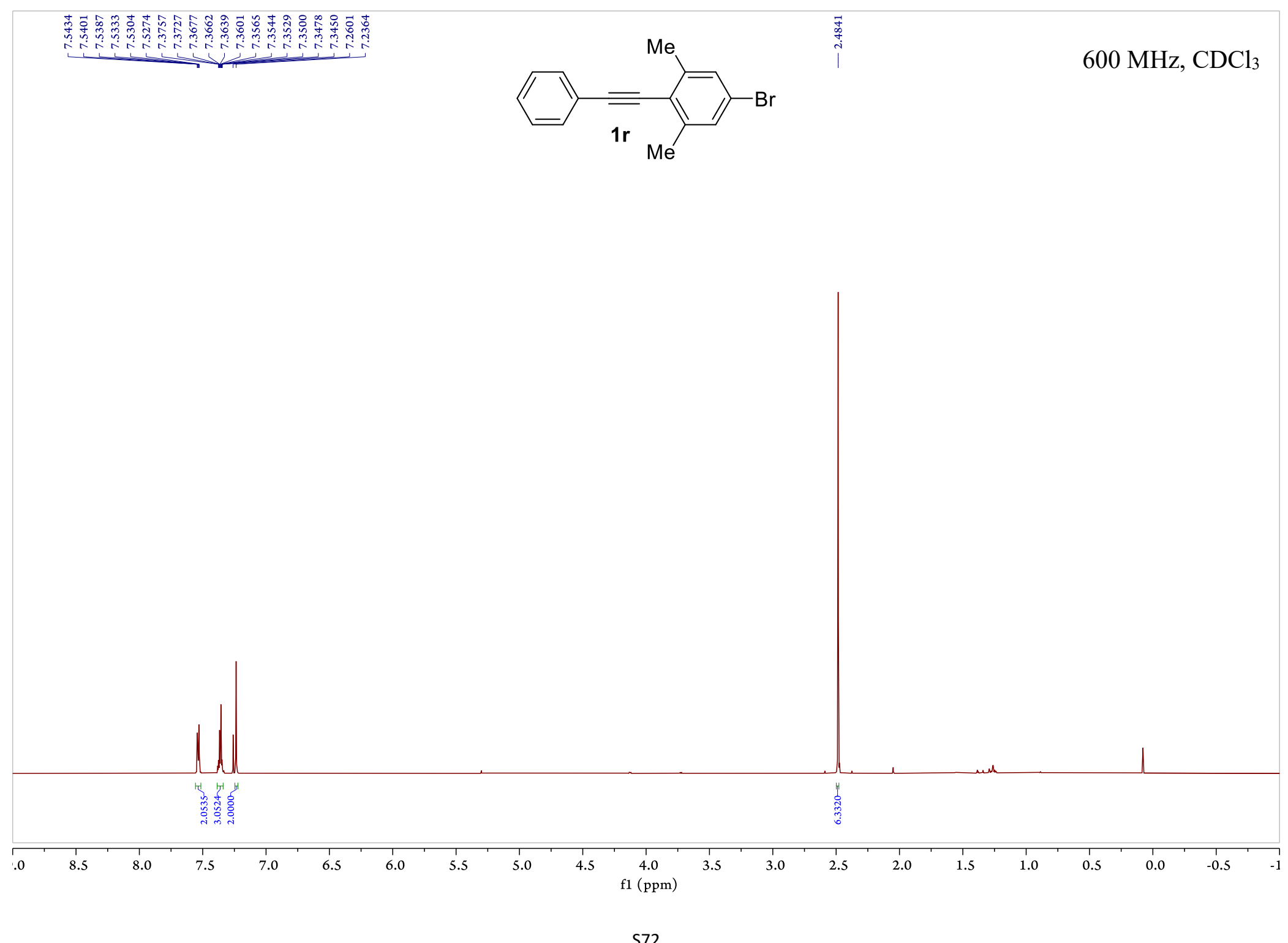




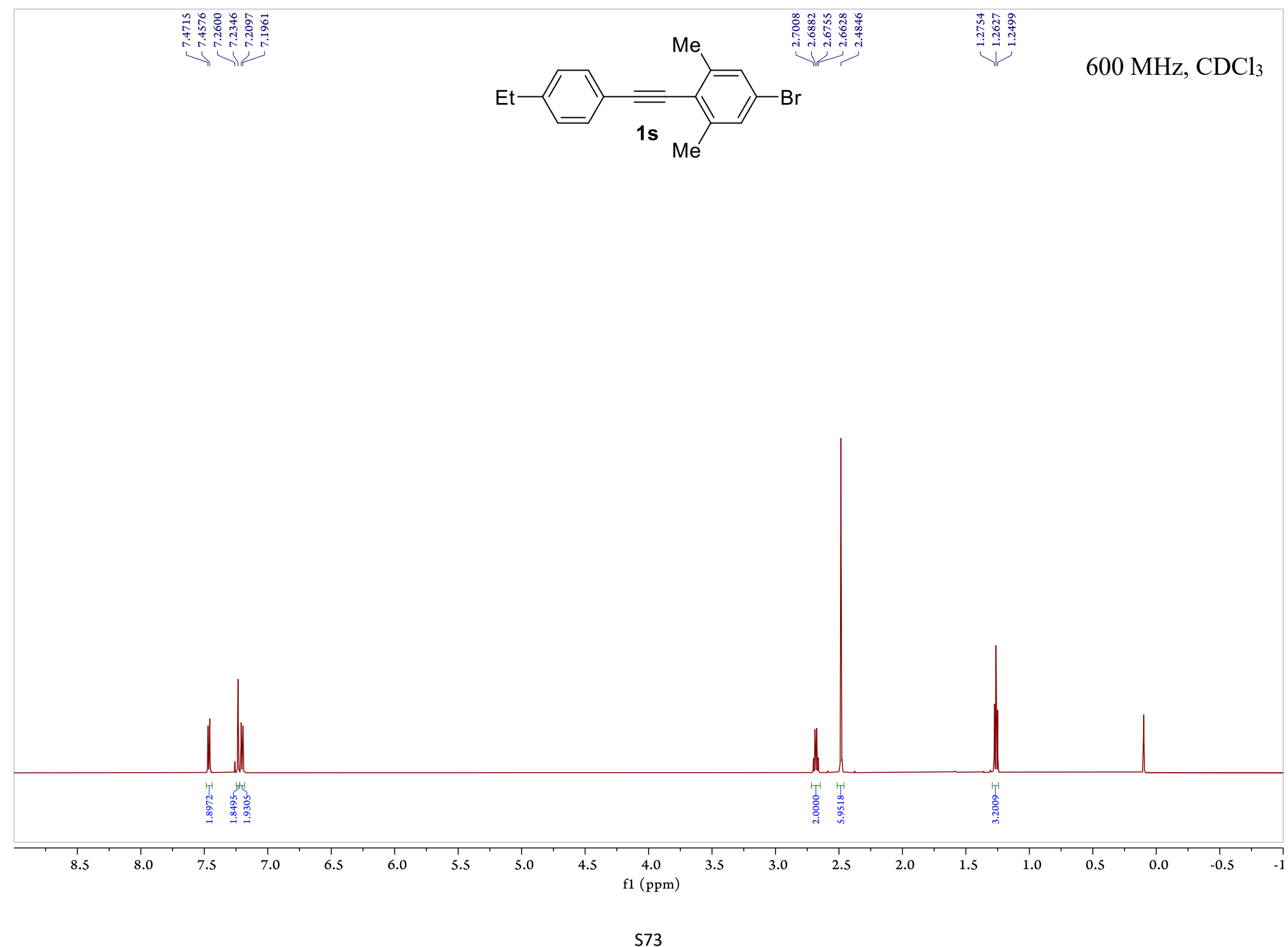




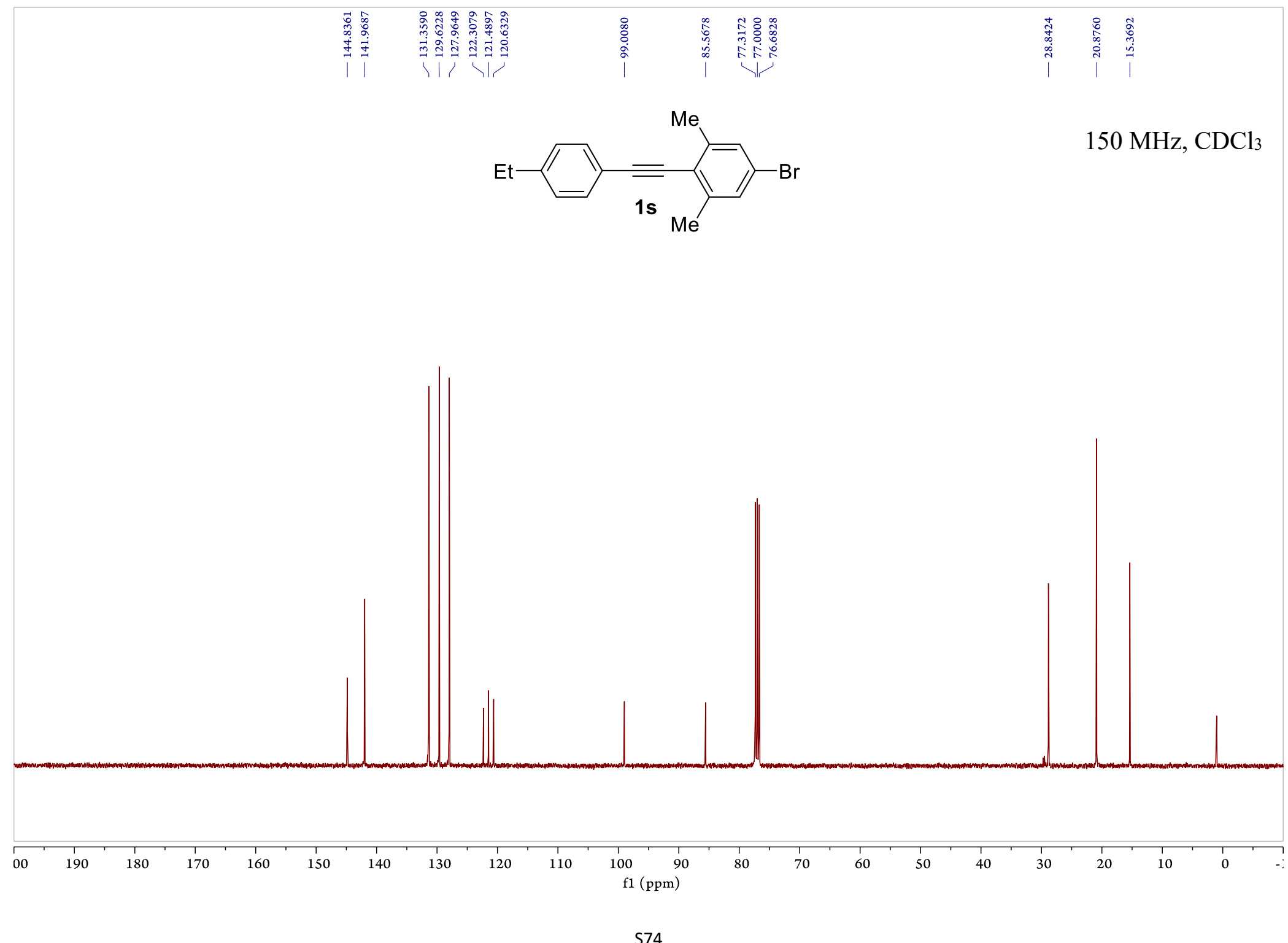




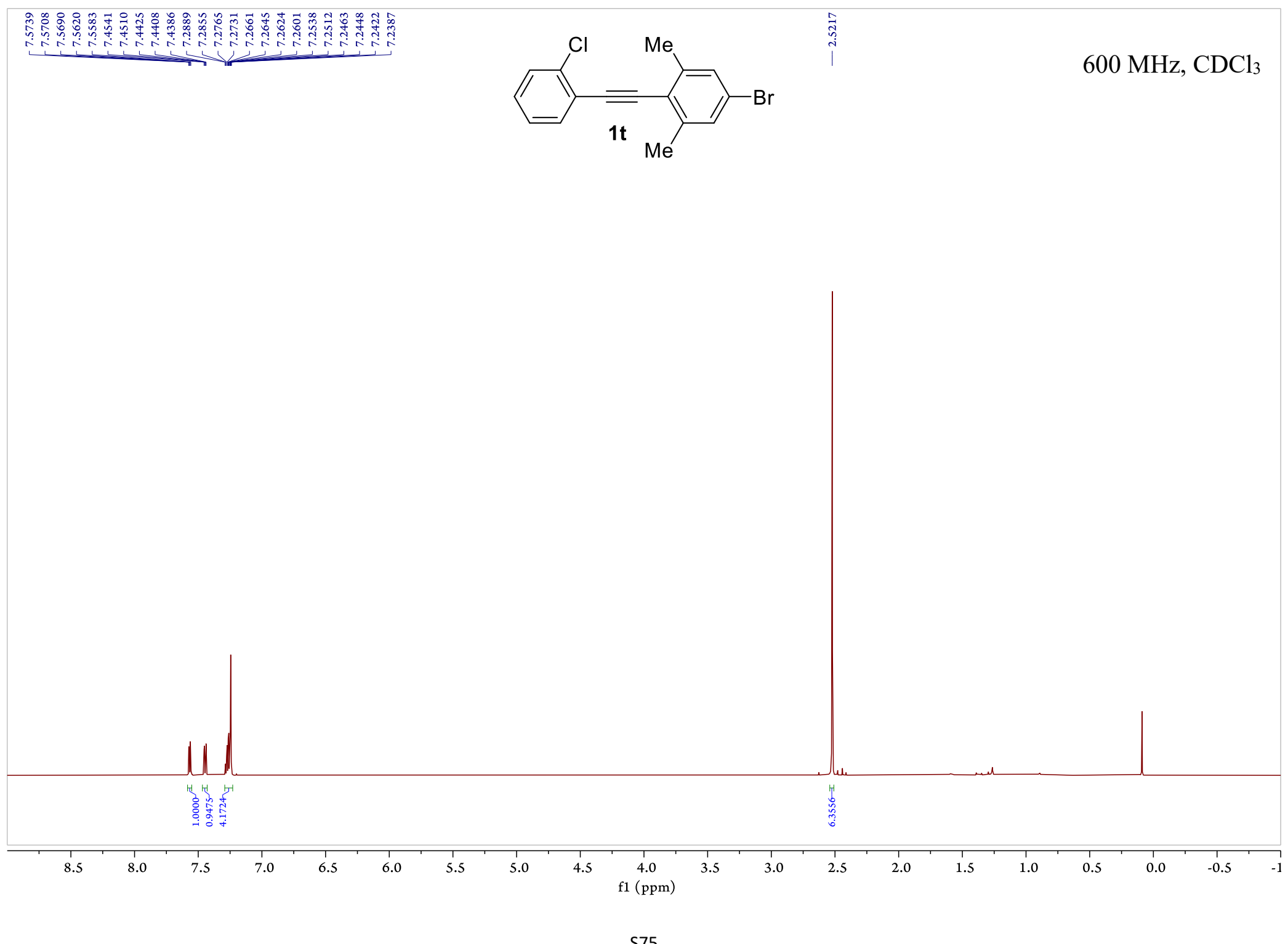




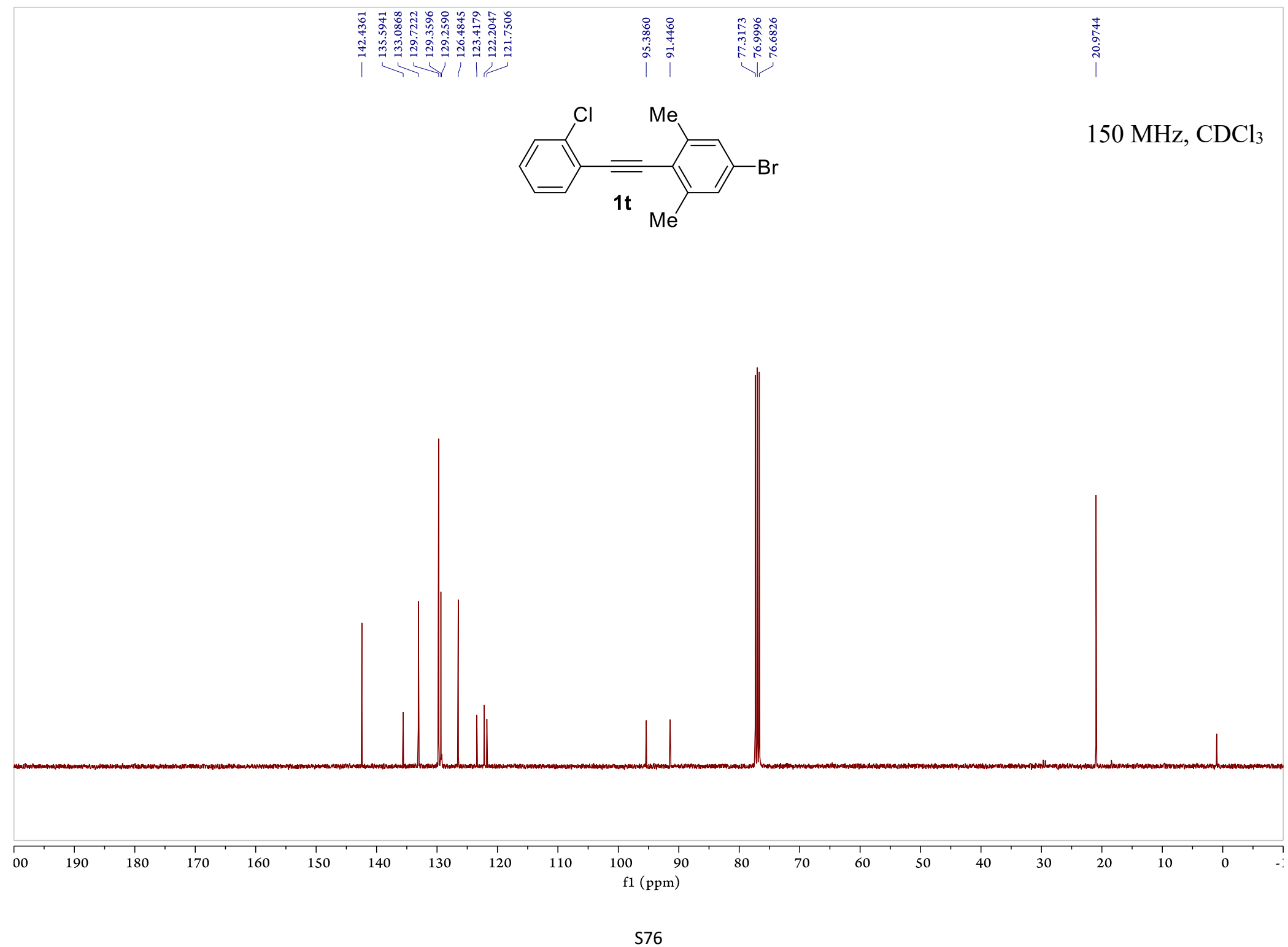




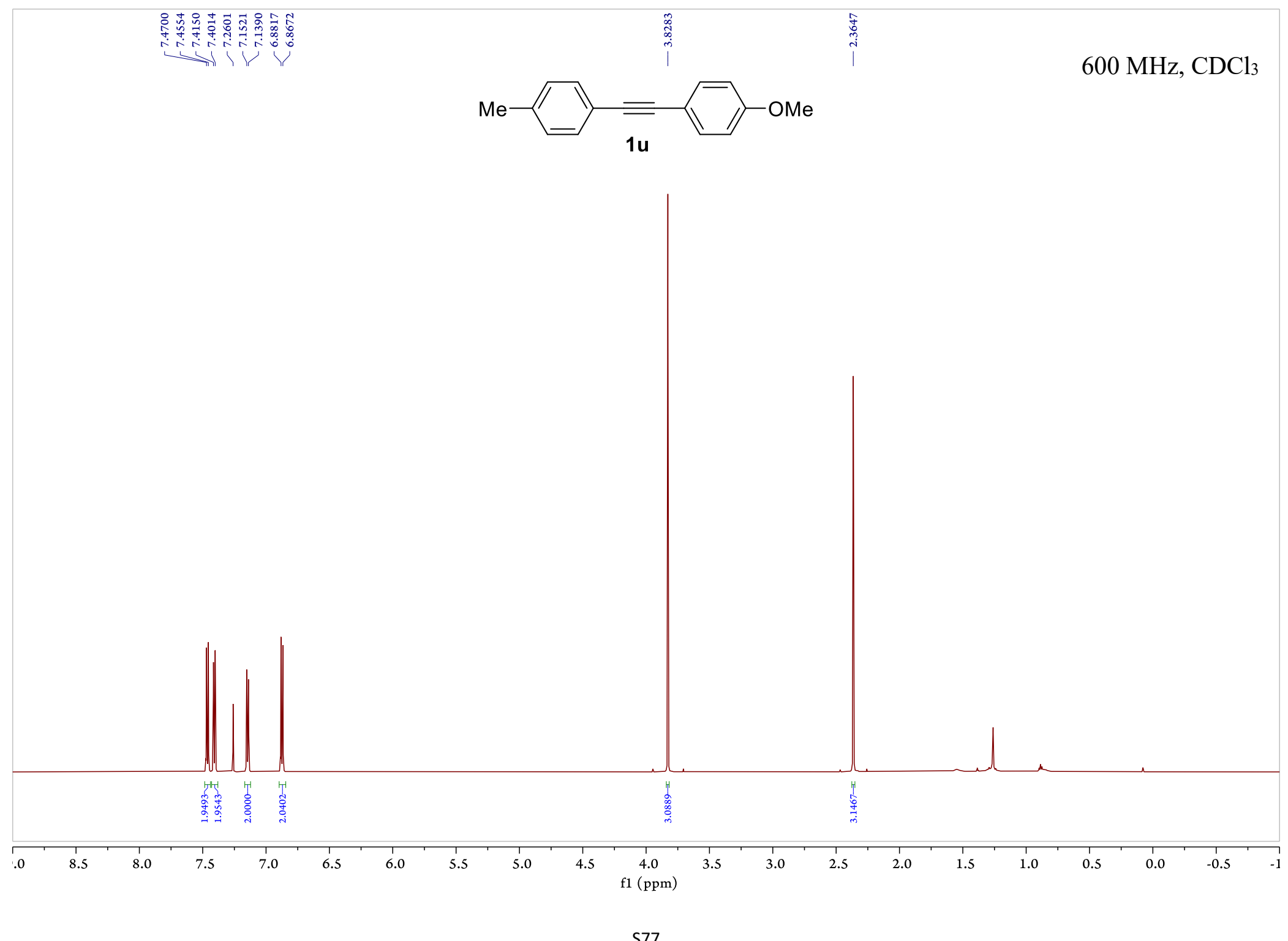




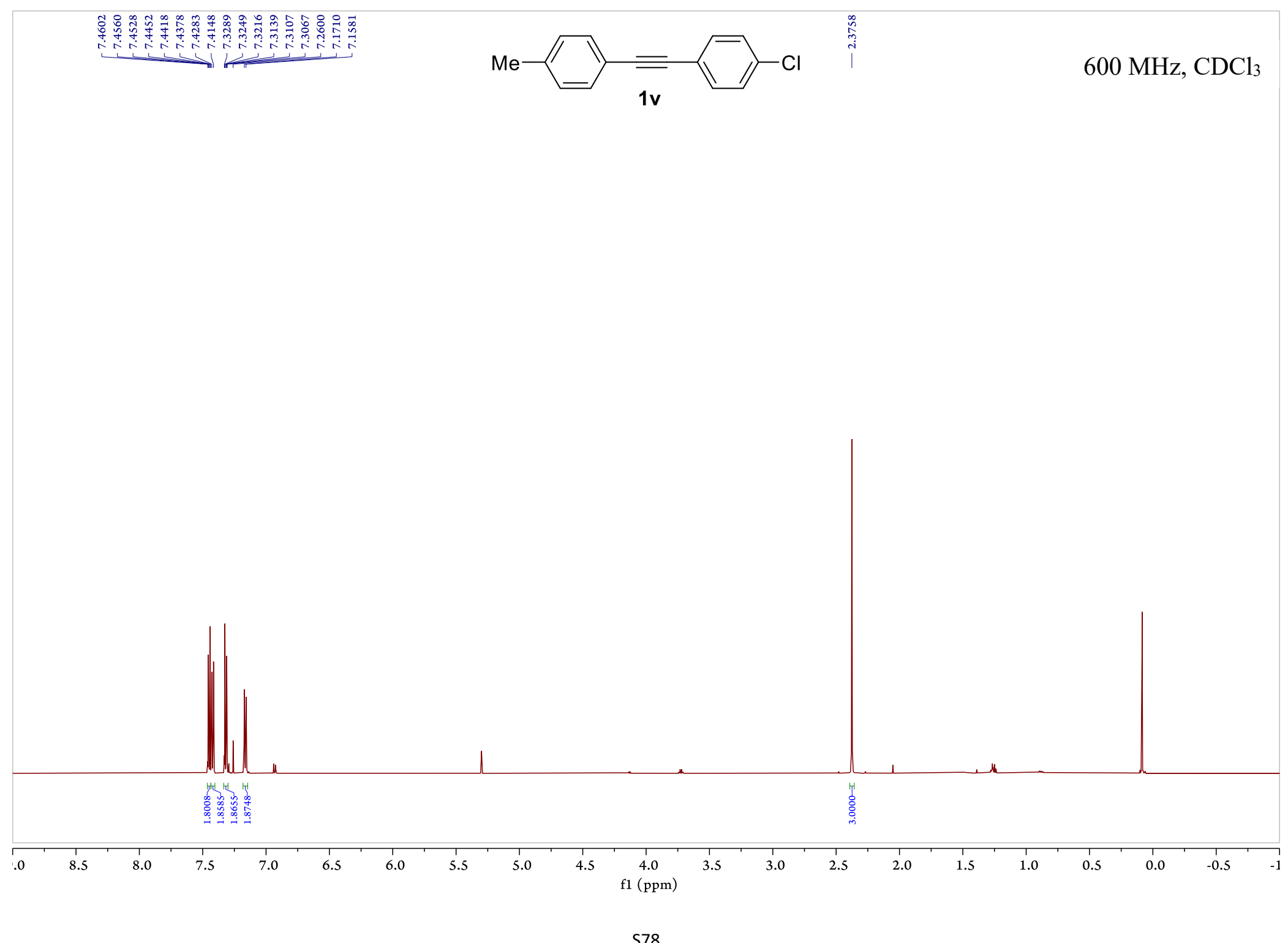




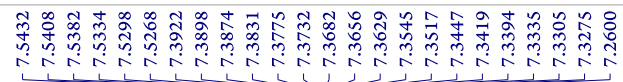

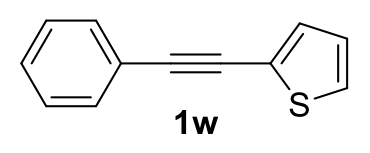

$600 \mathrm{MHz}, \mathrm{CDCl}_{3}$

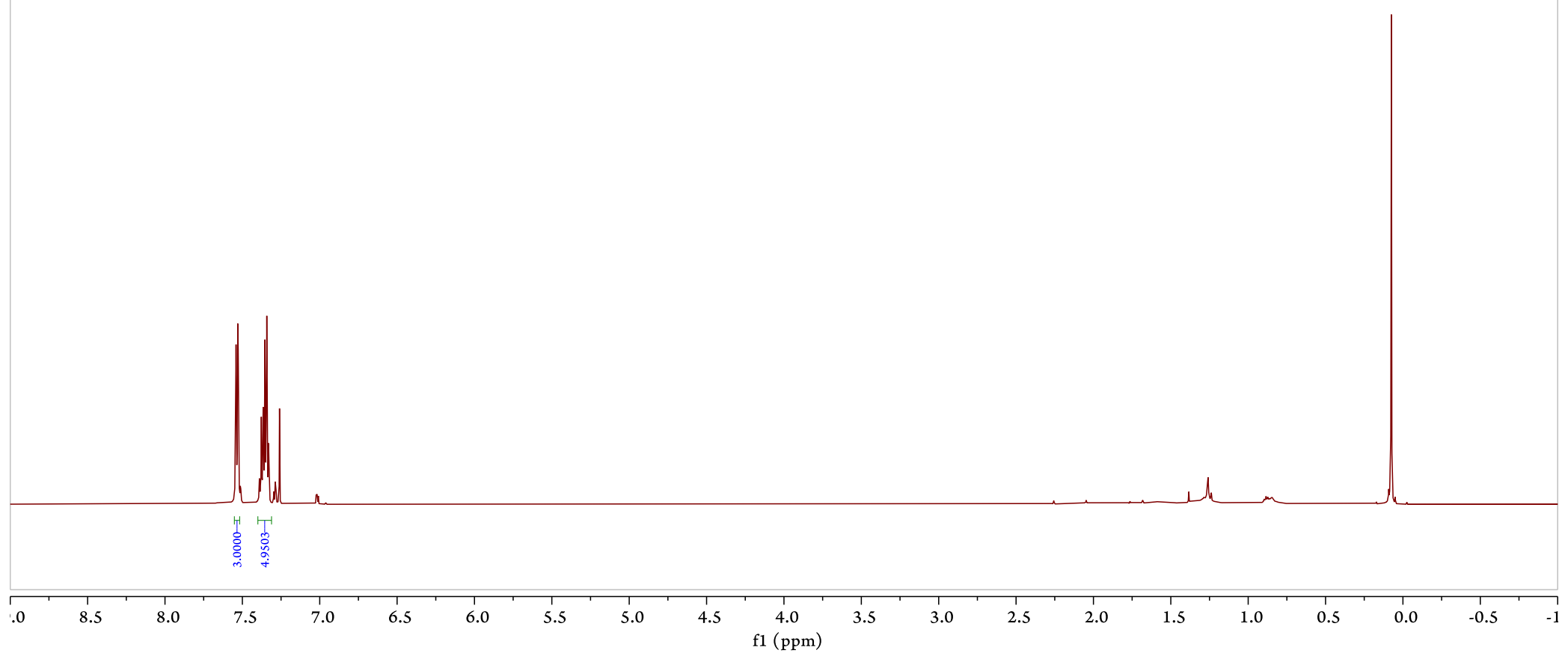

S79 
18. Copies of the ${ }^{1} \mathrm{H}$ and ${ }^{13} \mathrm{C}$ NMR for compounds 3-13 and CWJ-a-5

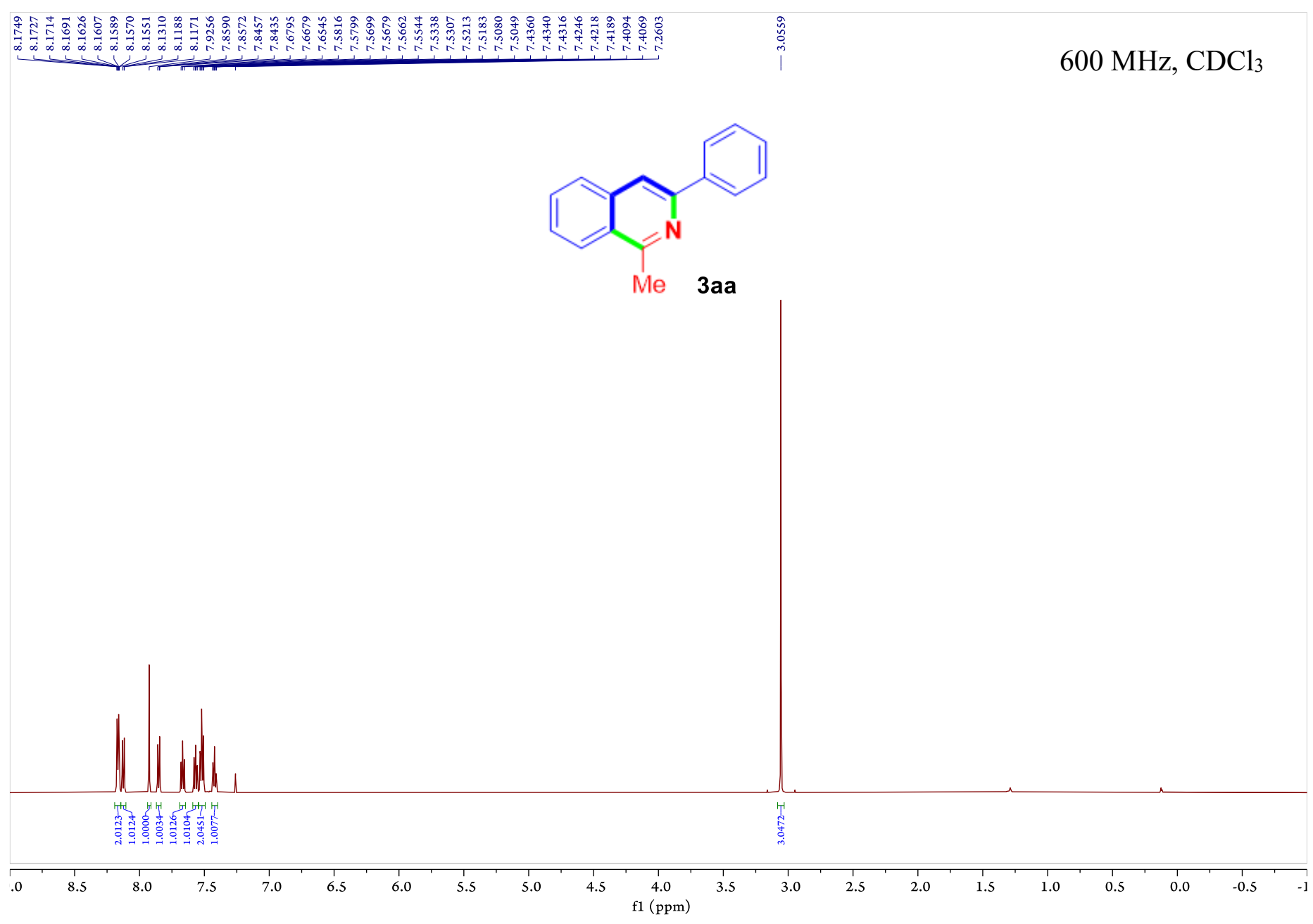

580 


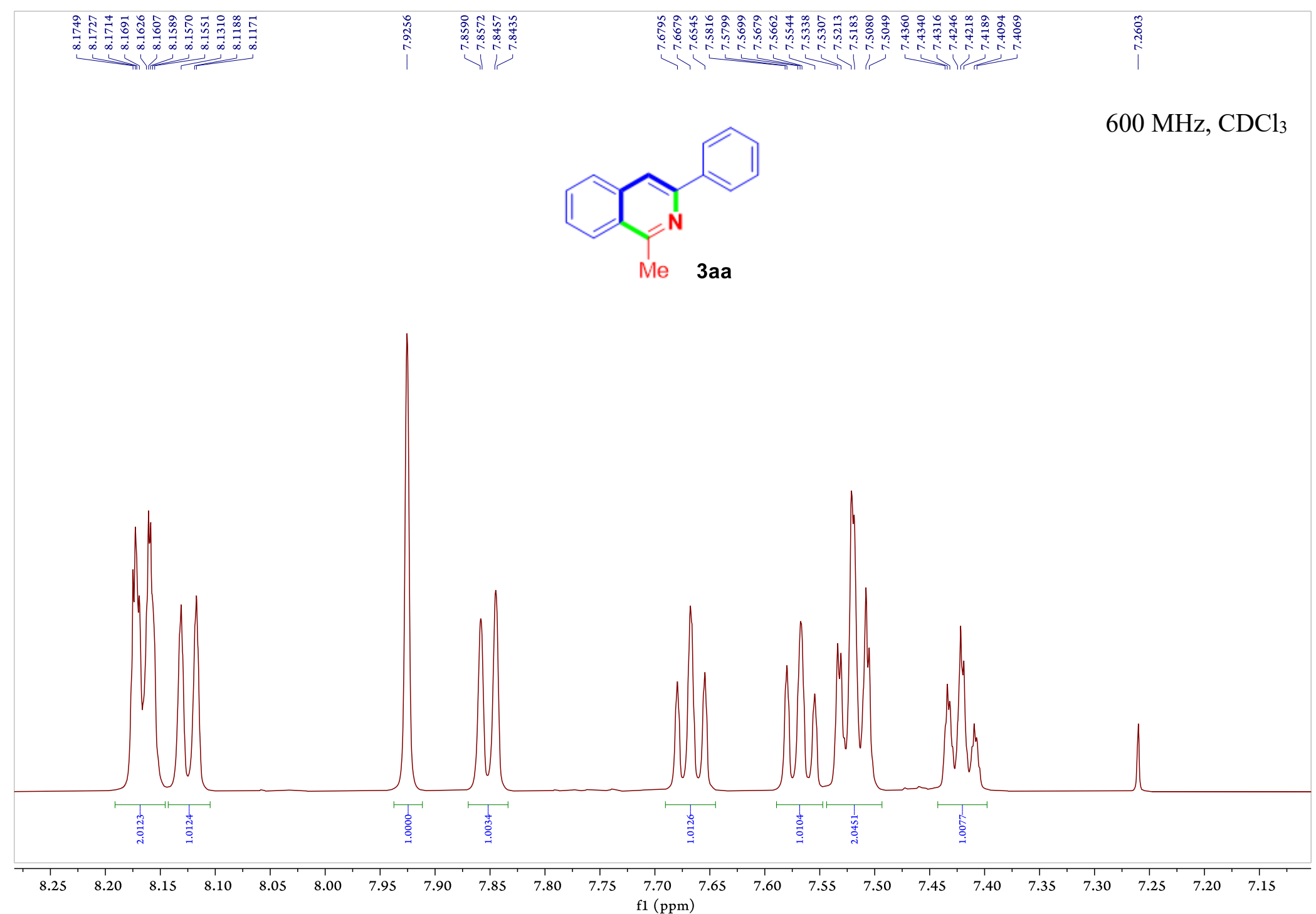

S81 


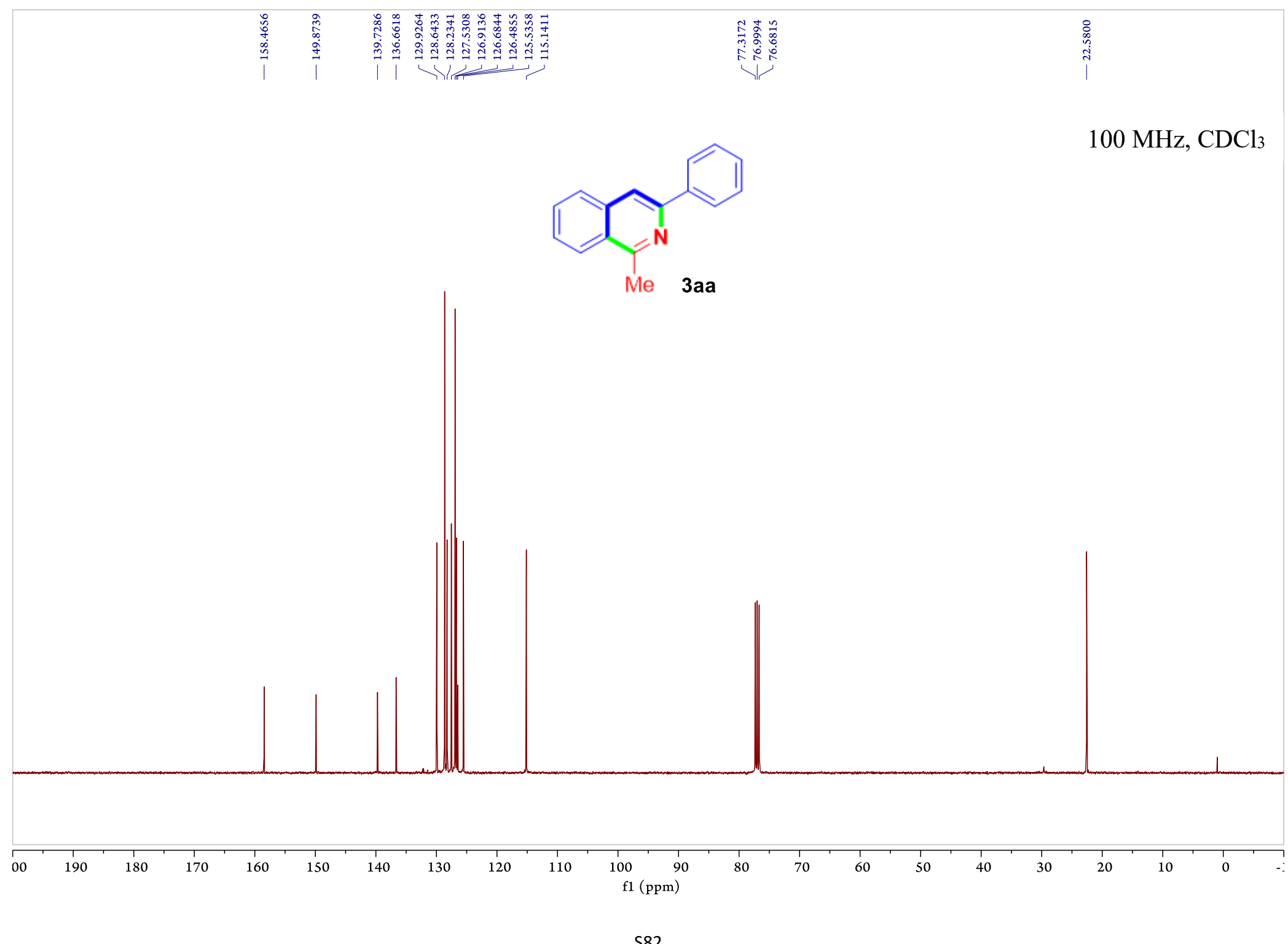




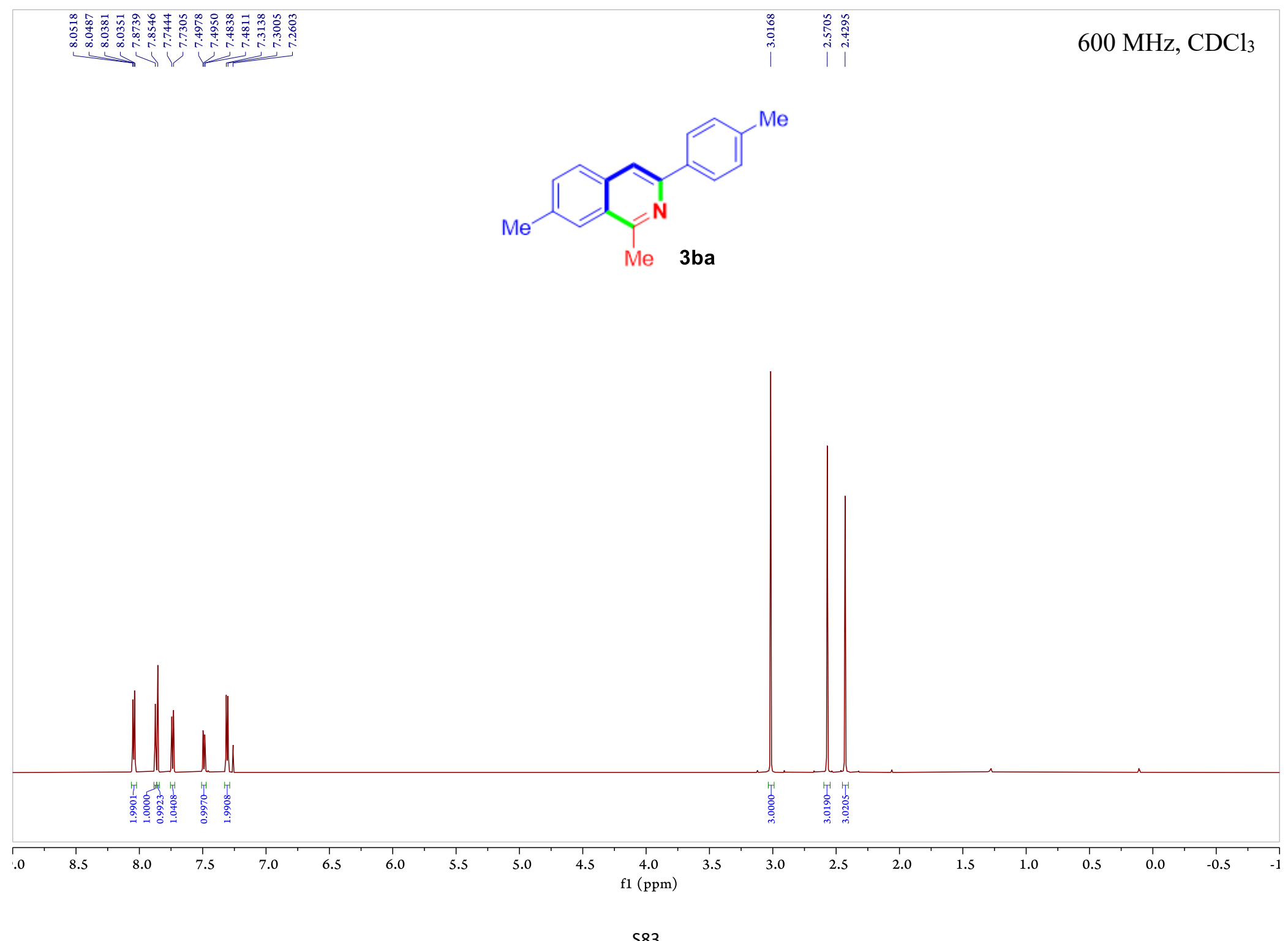

S83 


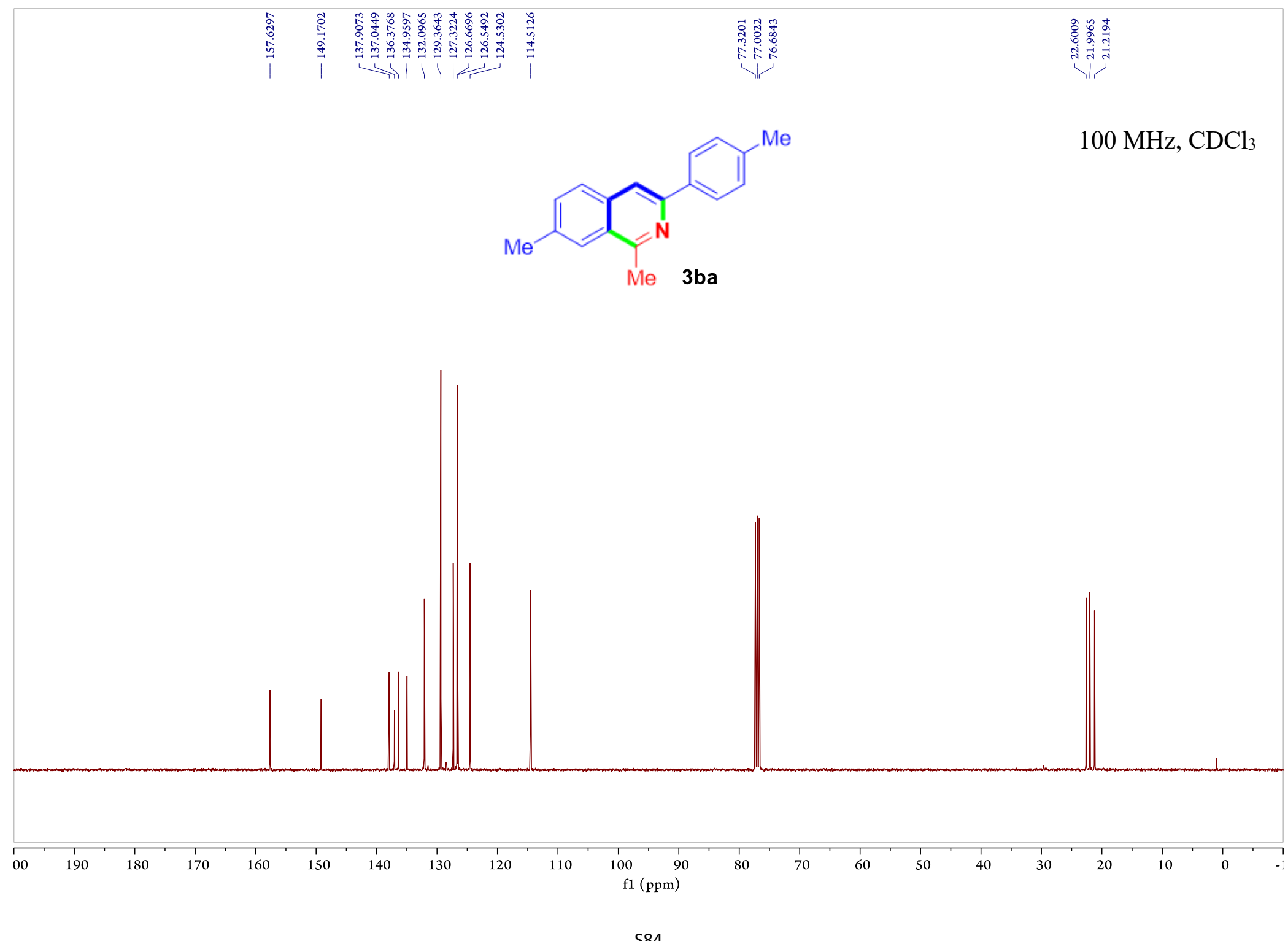




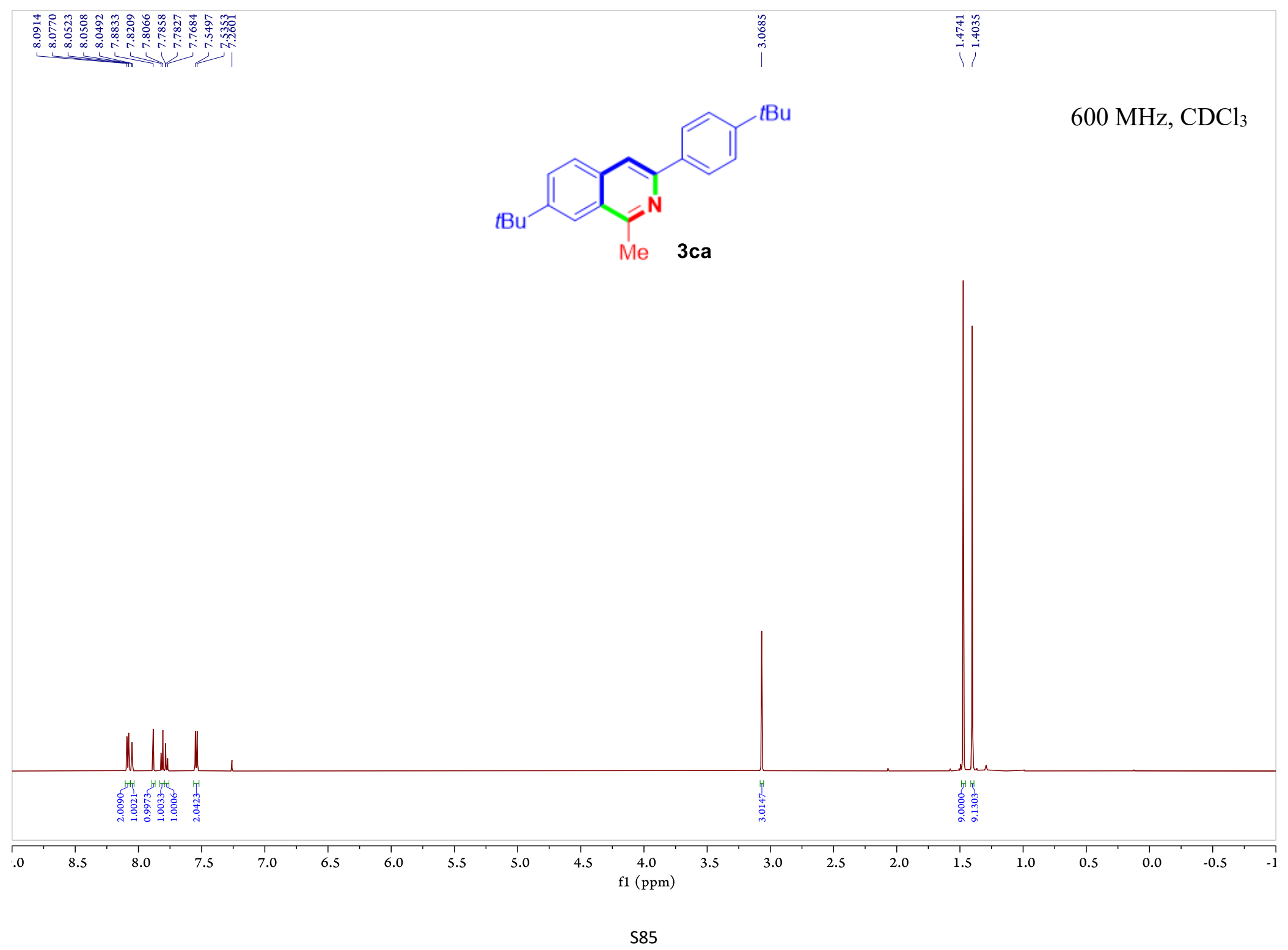




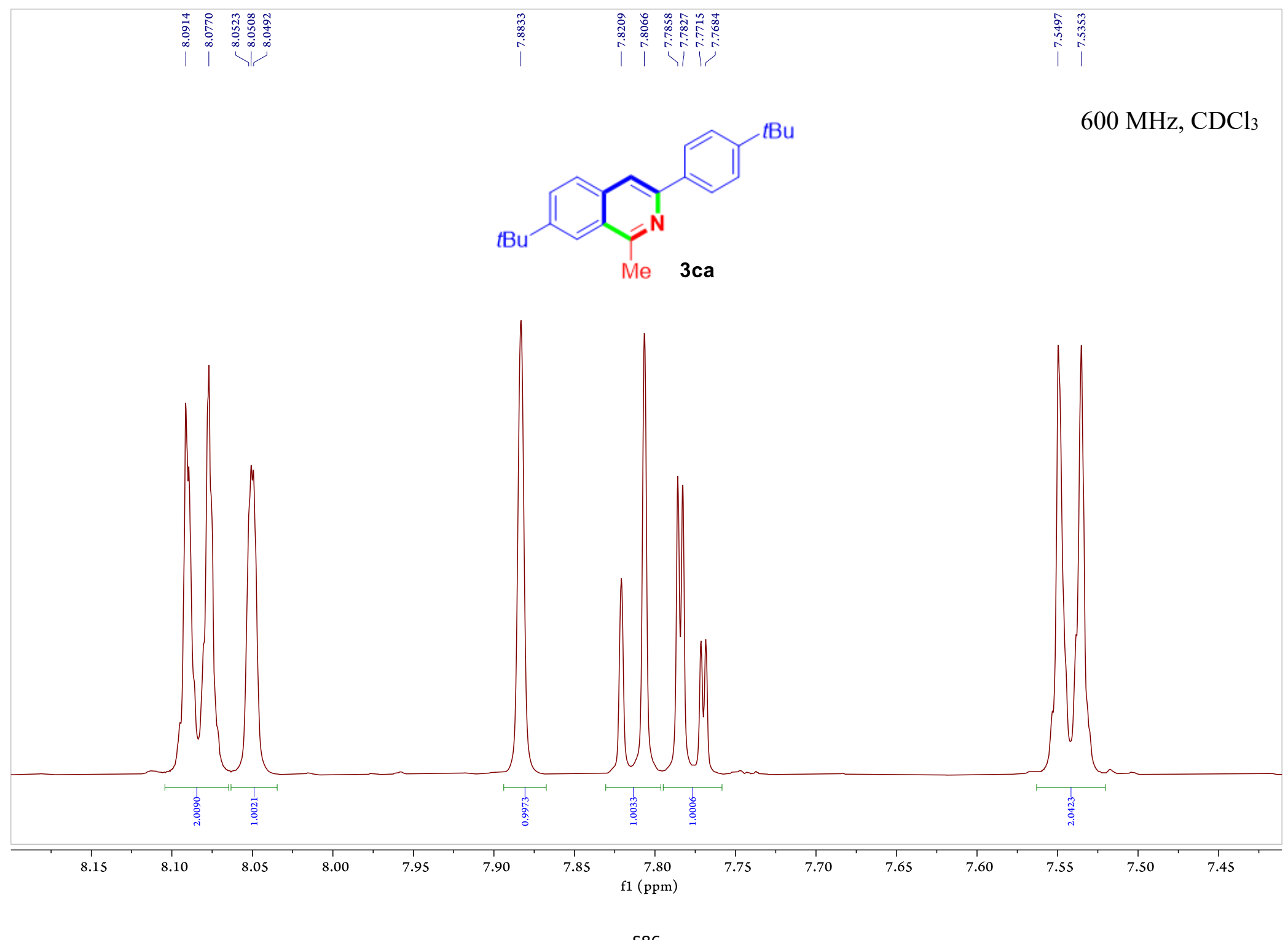




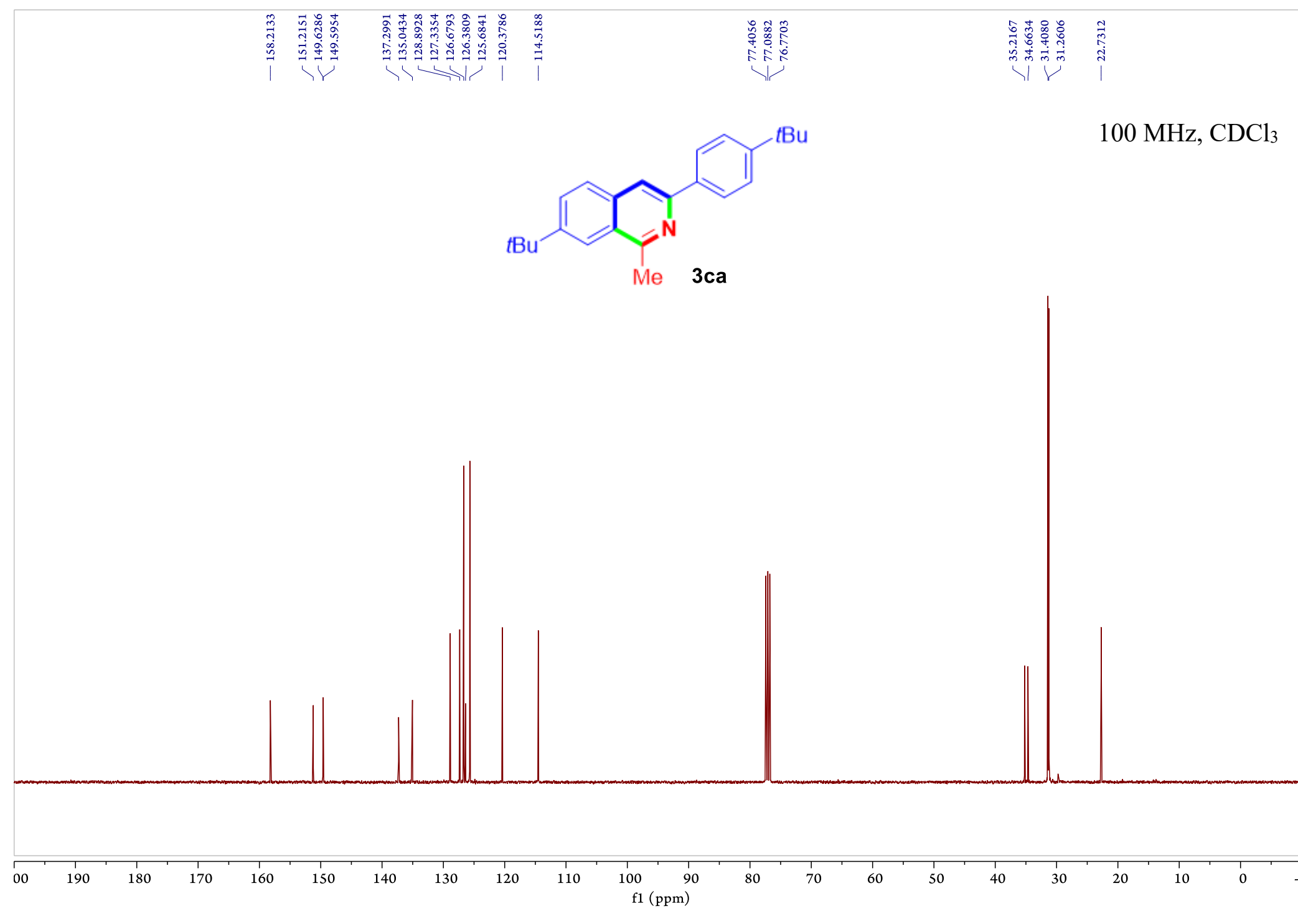




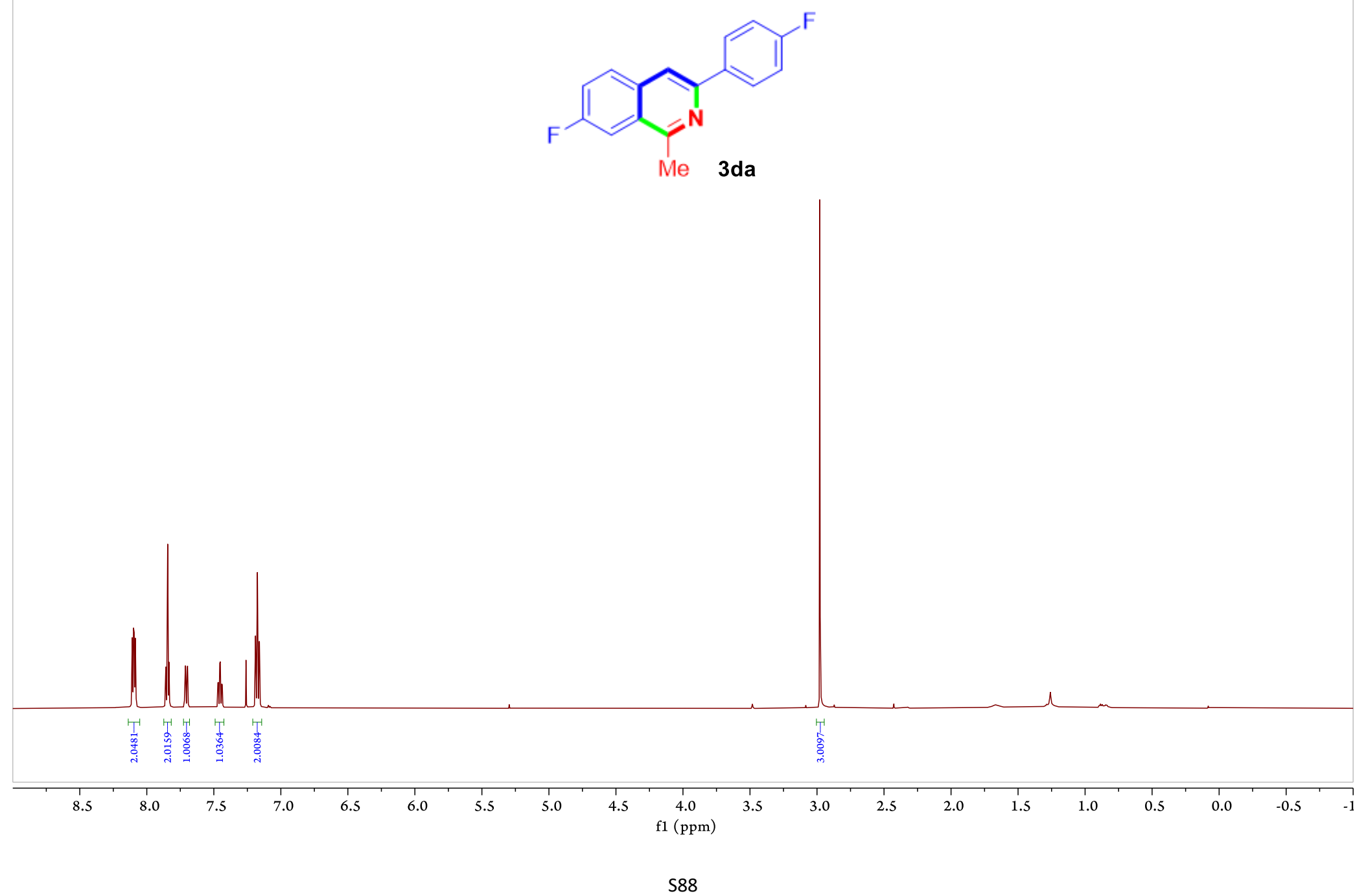




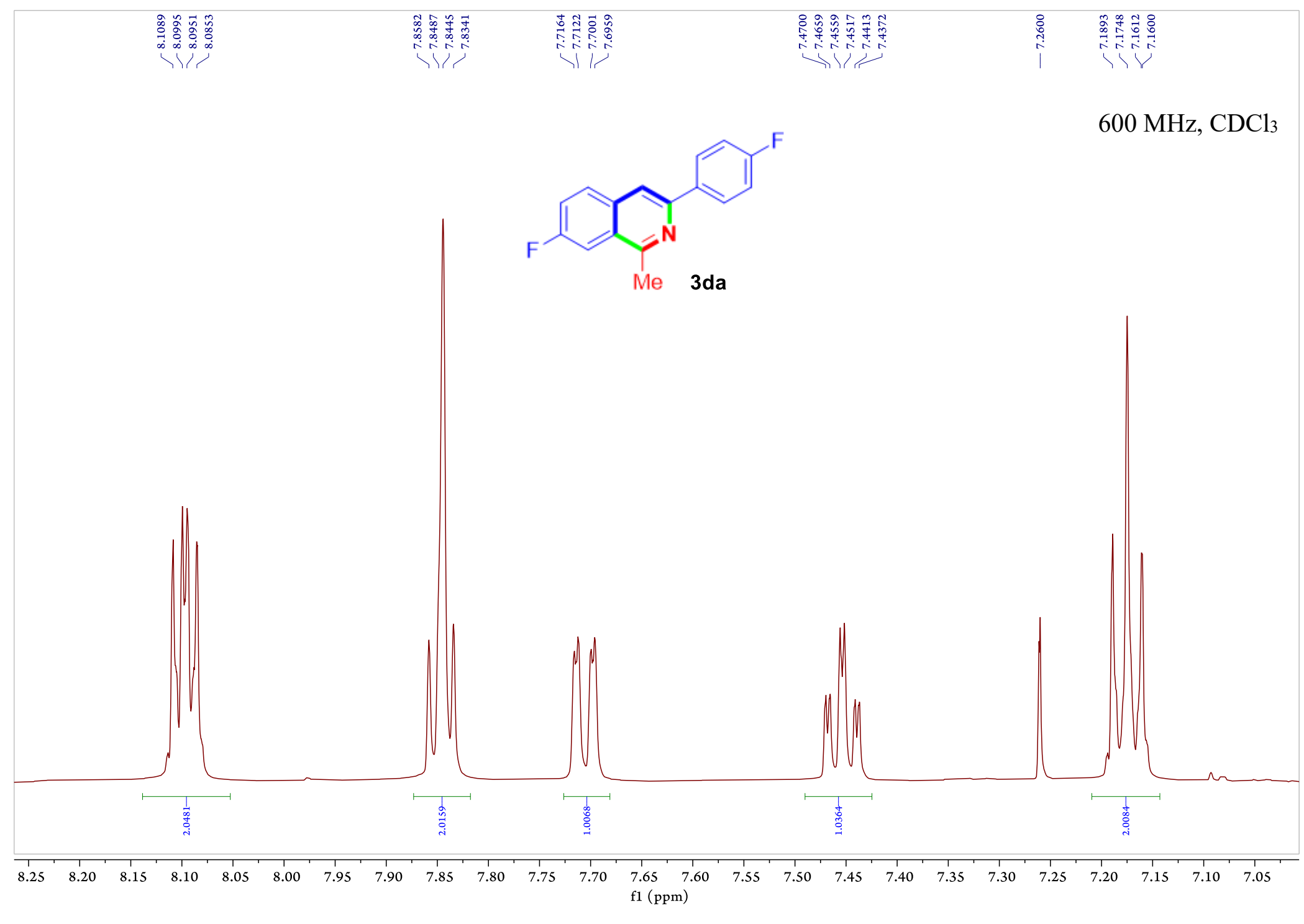

589 


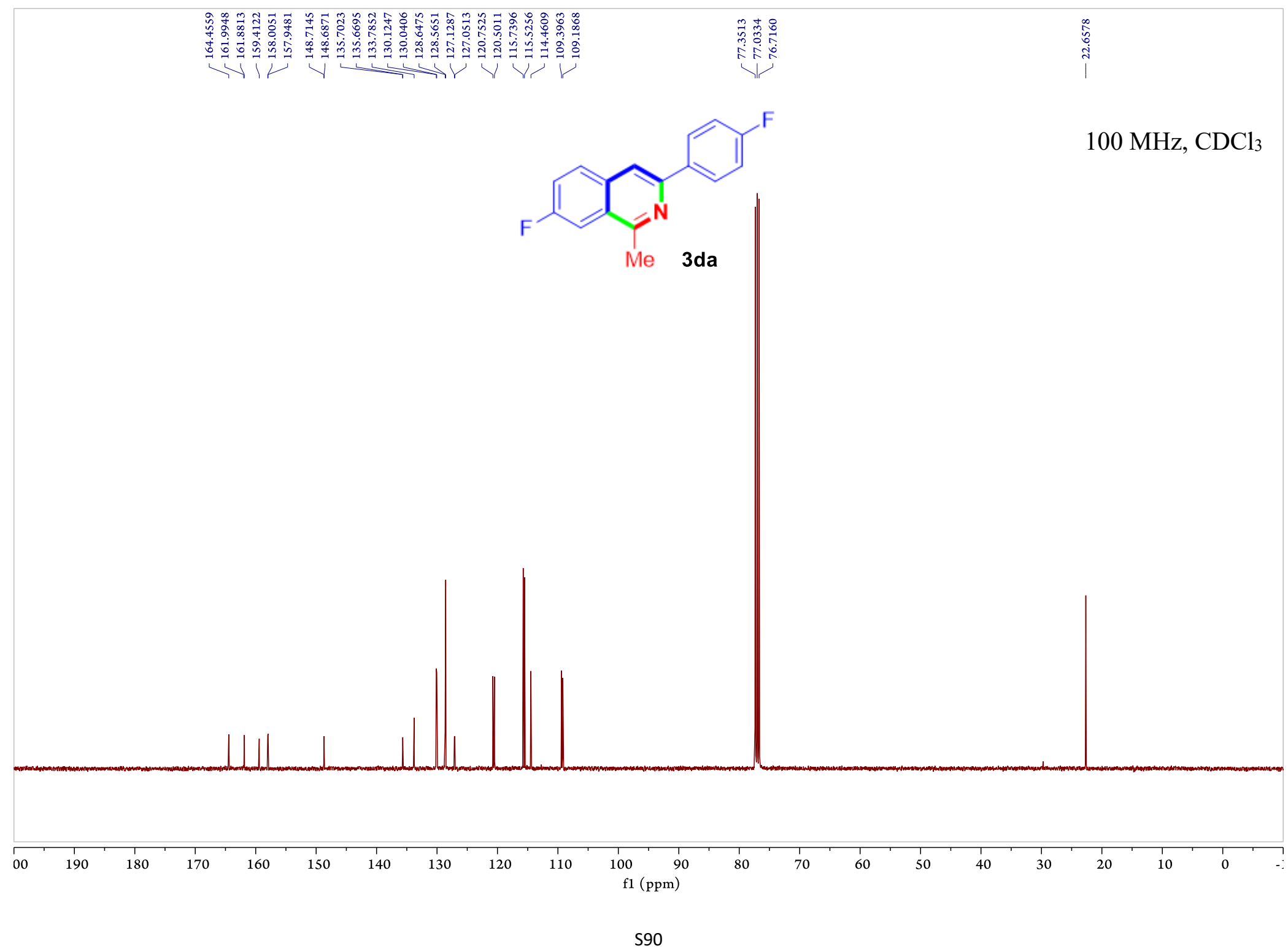




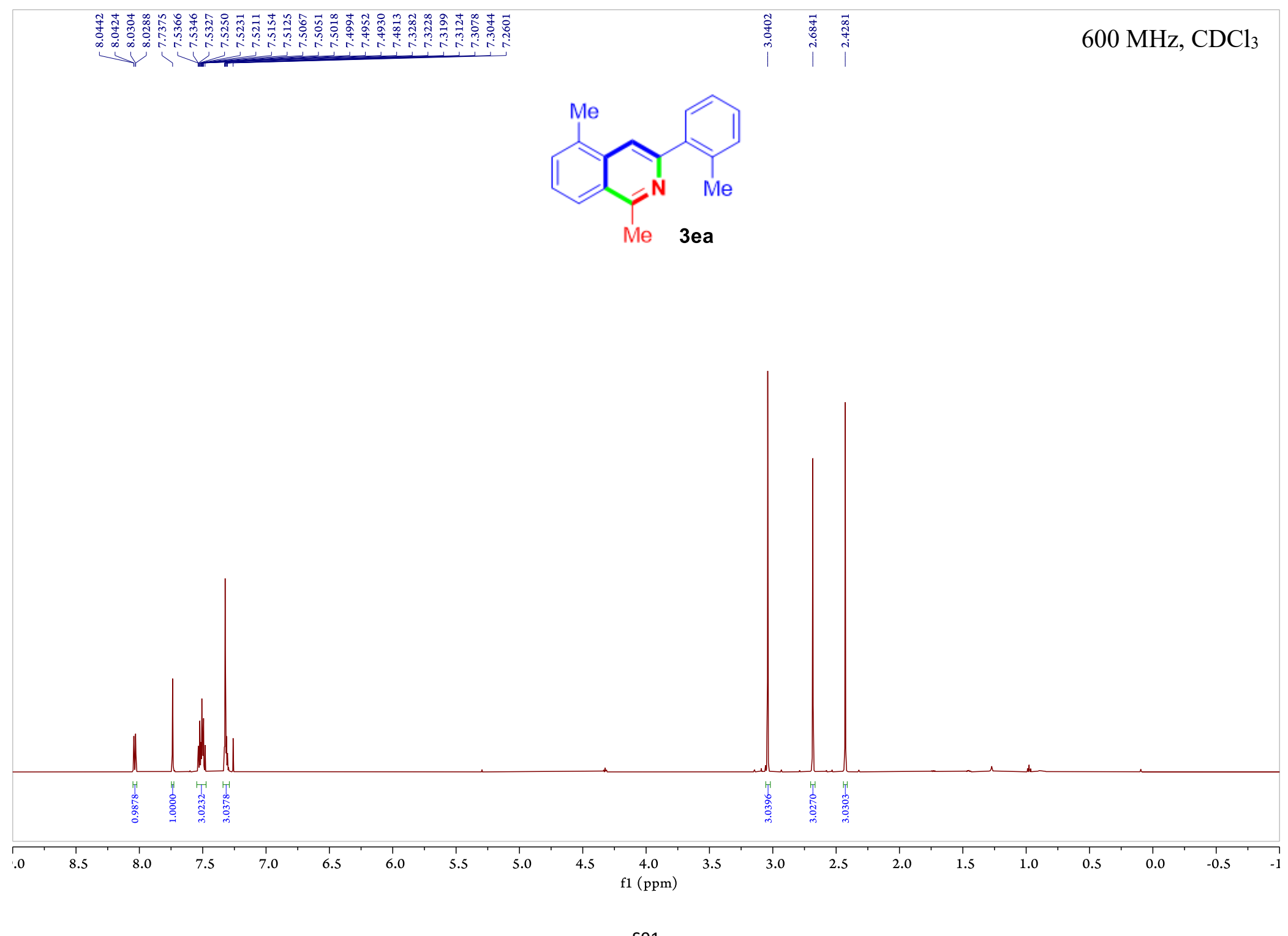

S91 


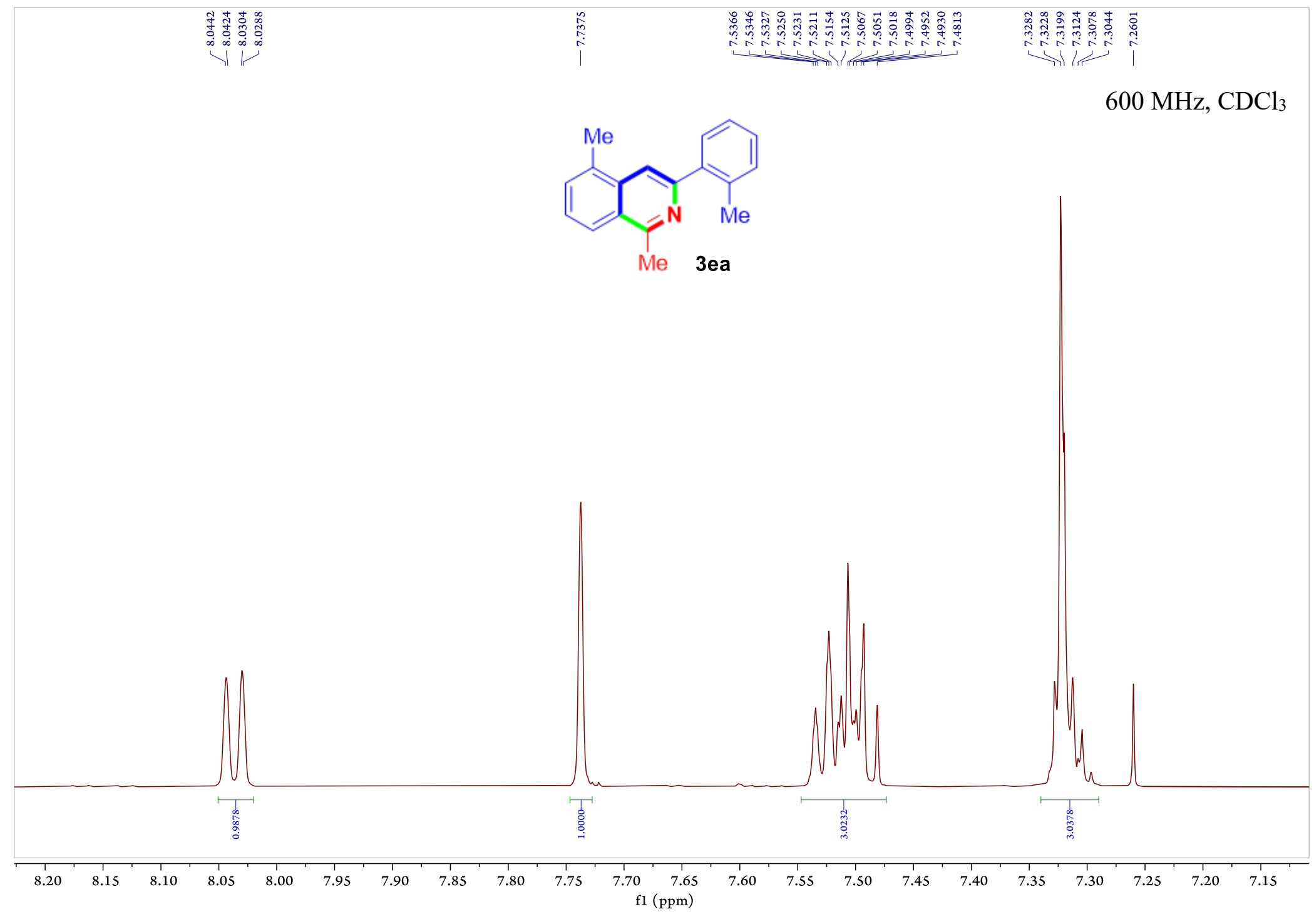

S92 

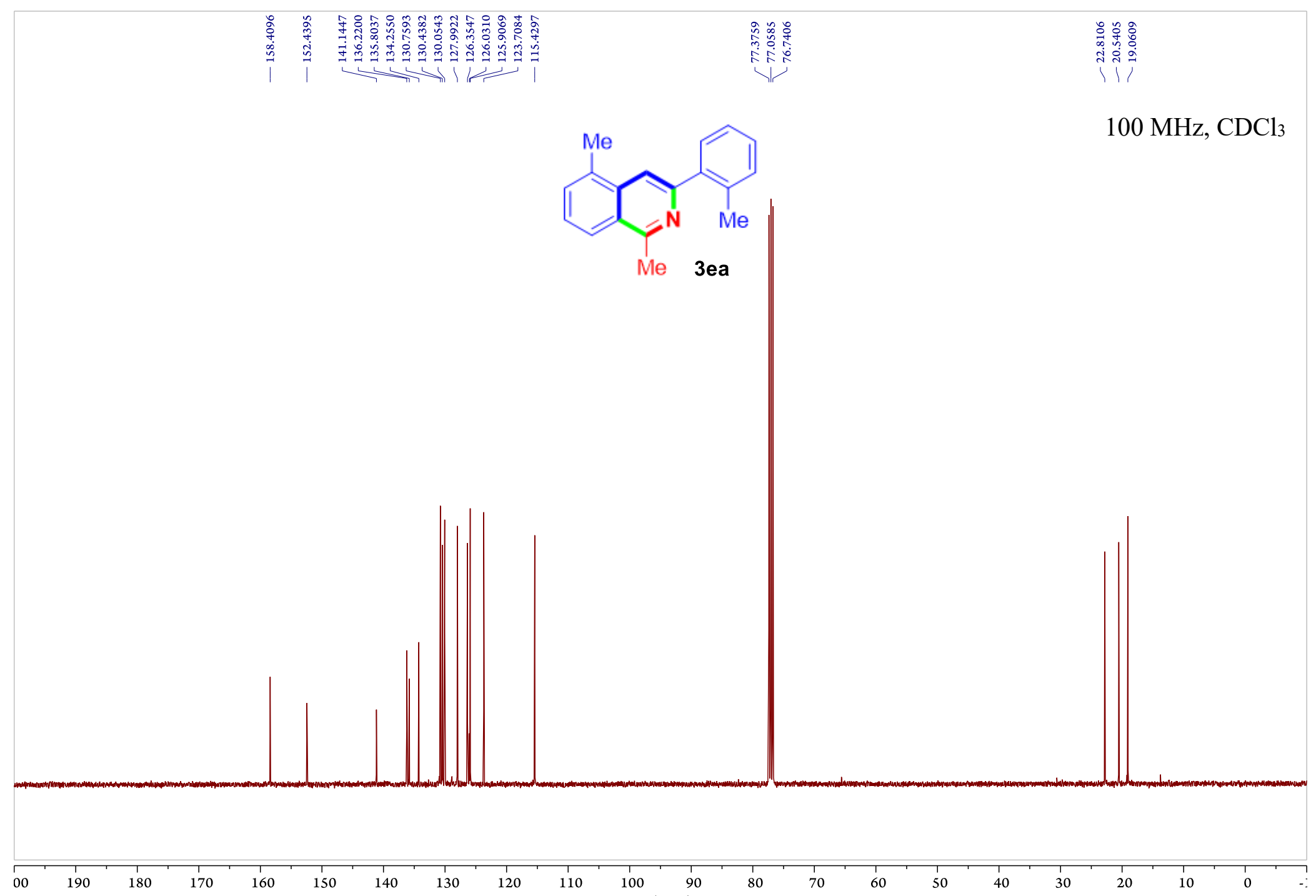

f1 (ppm) 


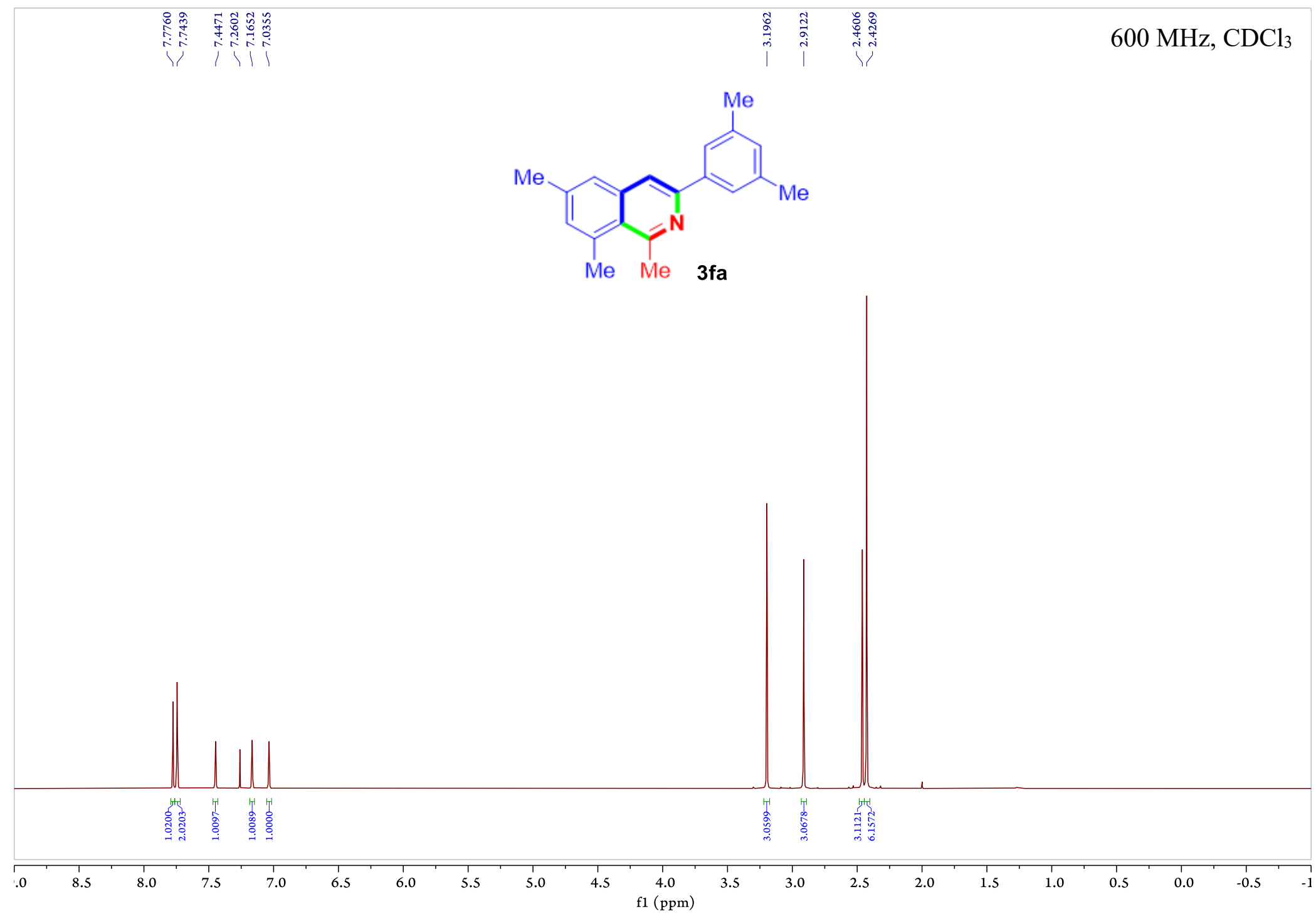

S94 


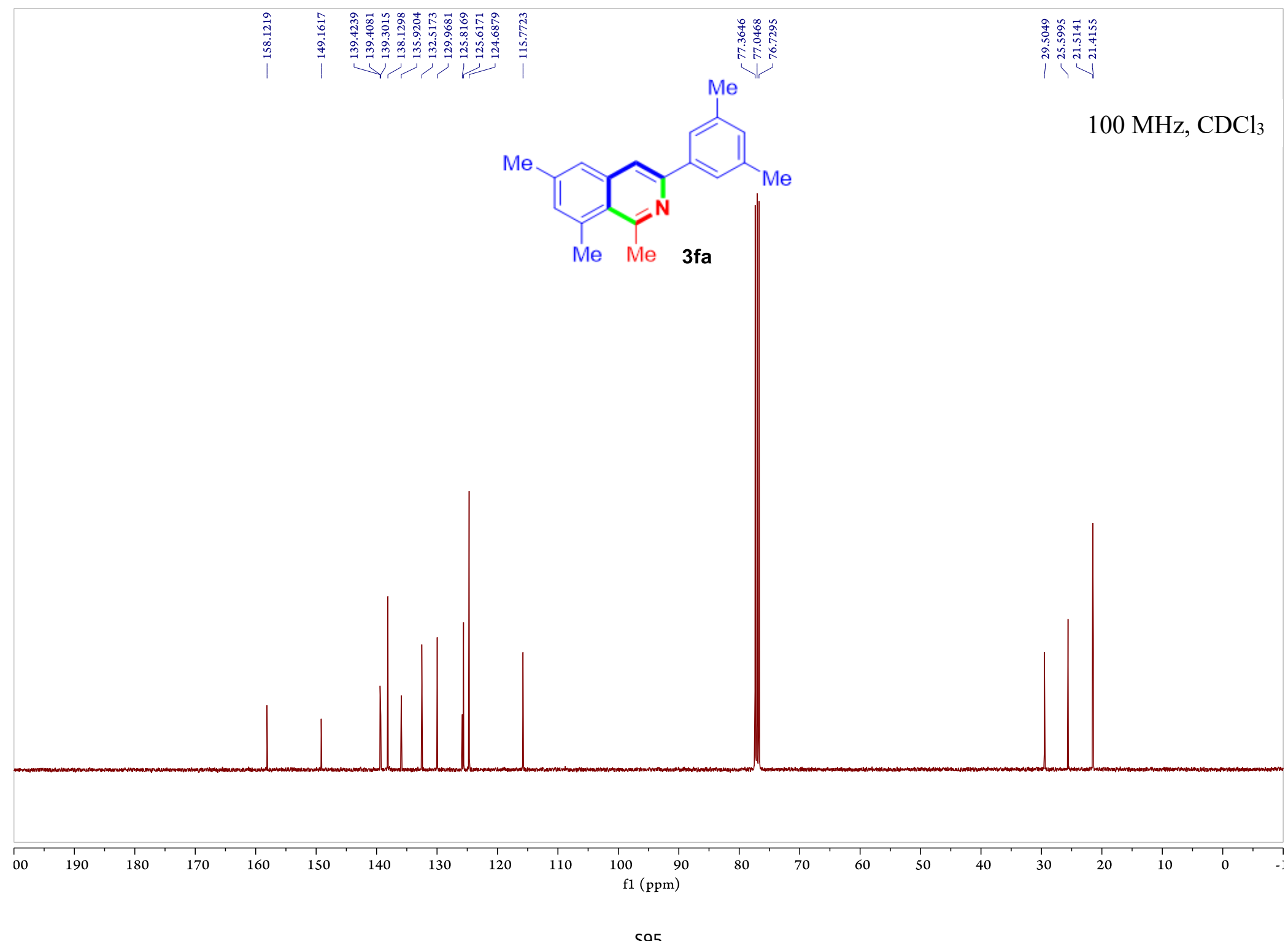



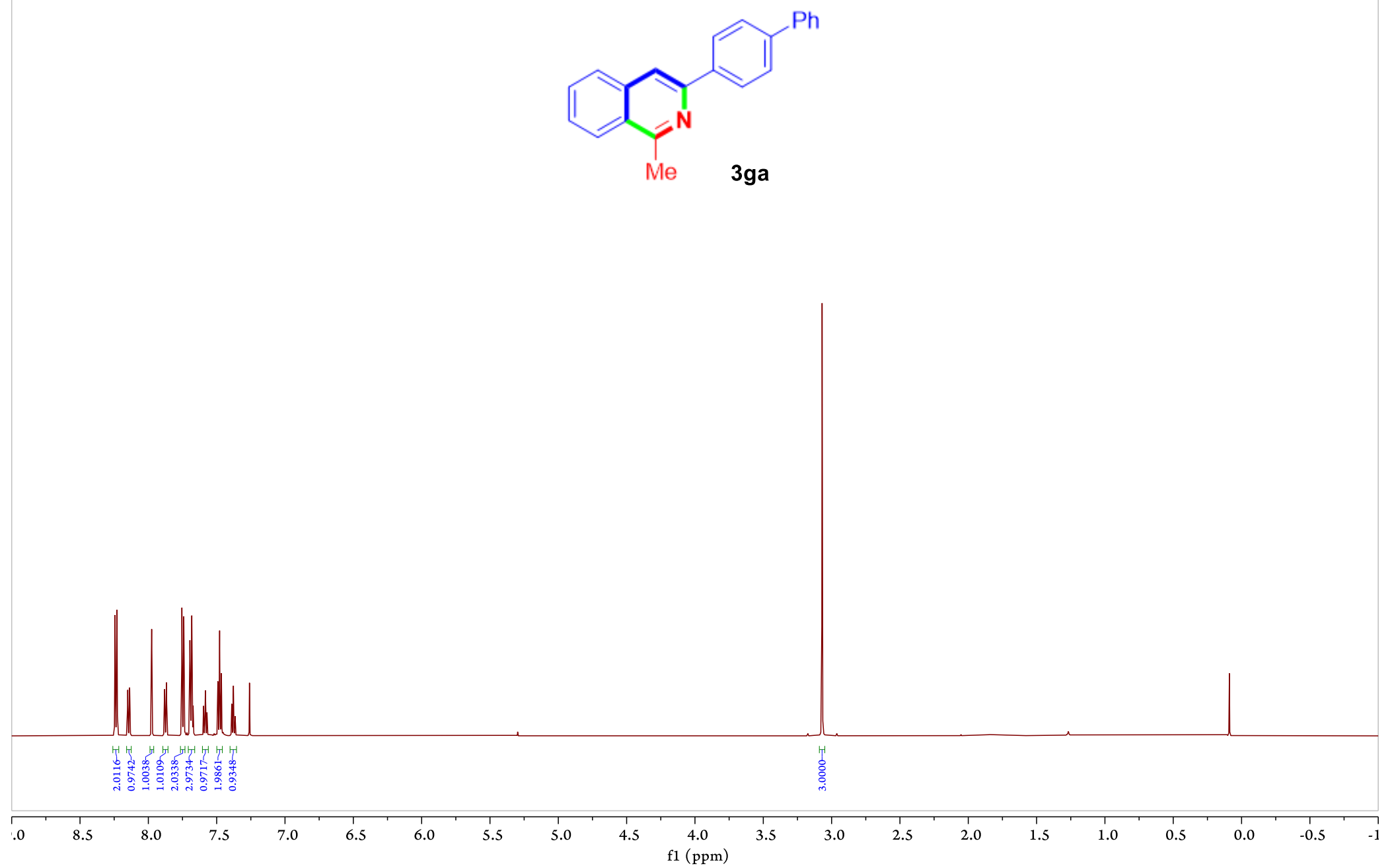

S96 


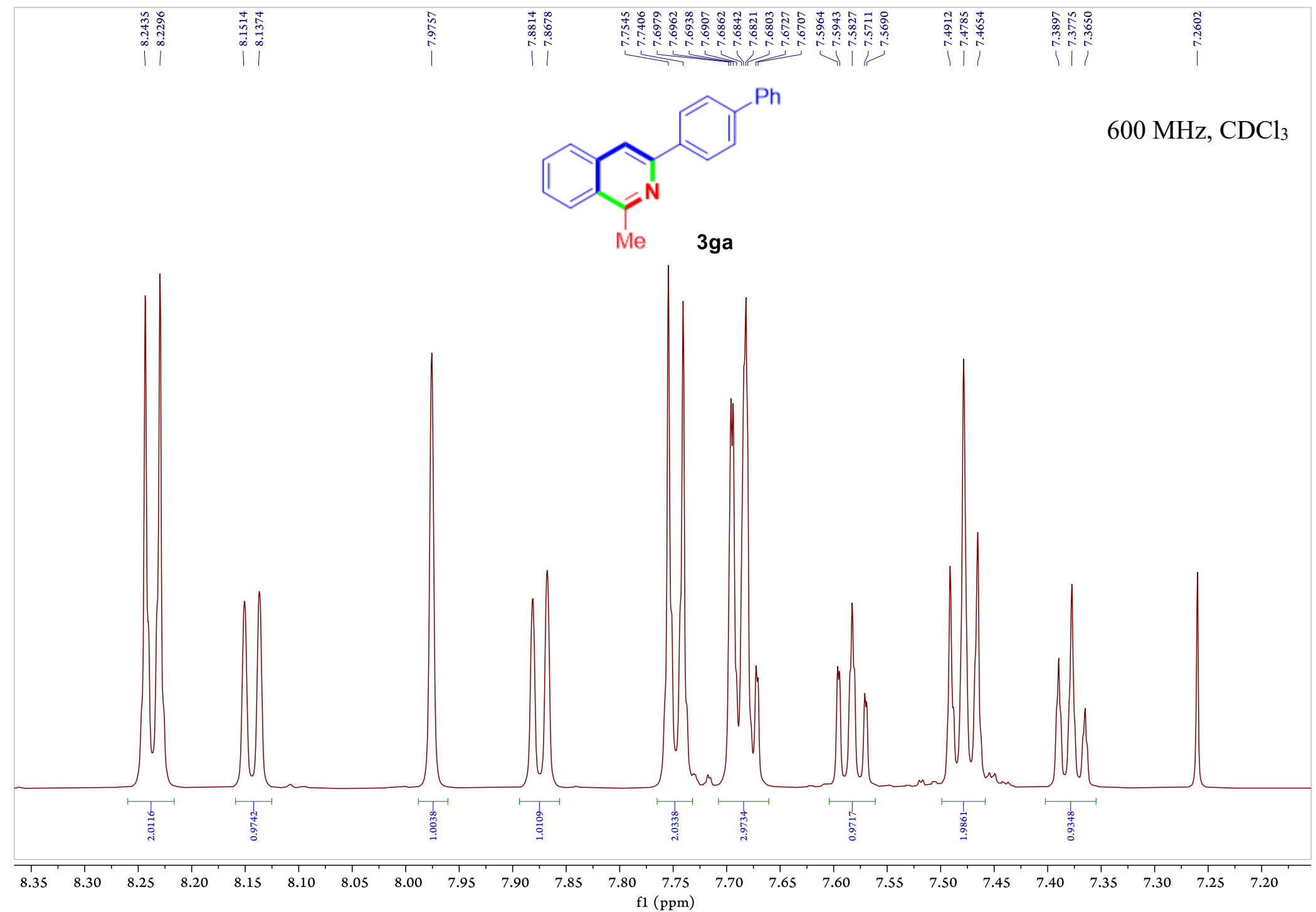

597 


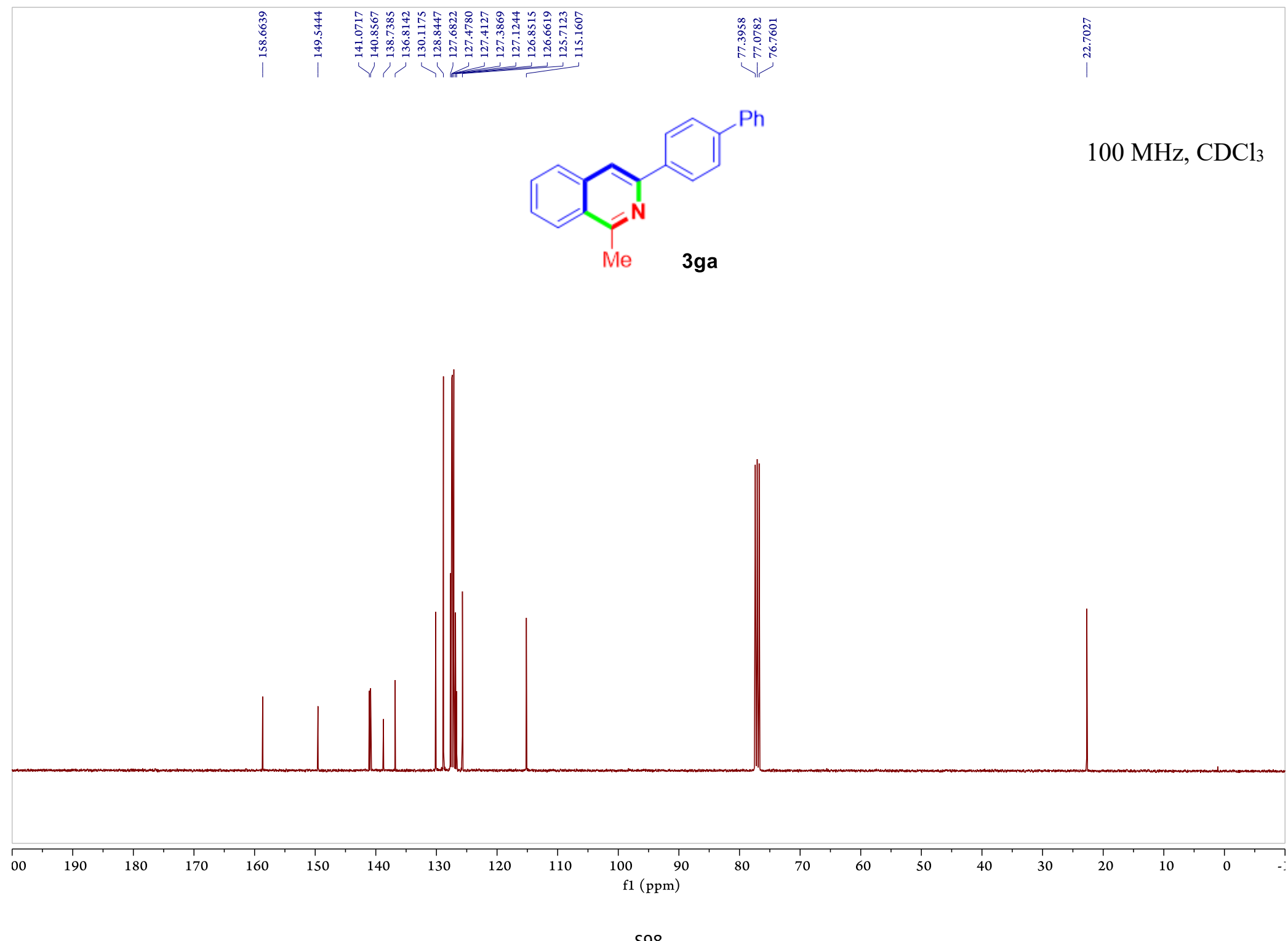



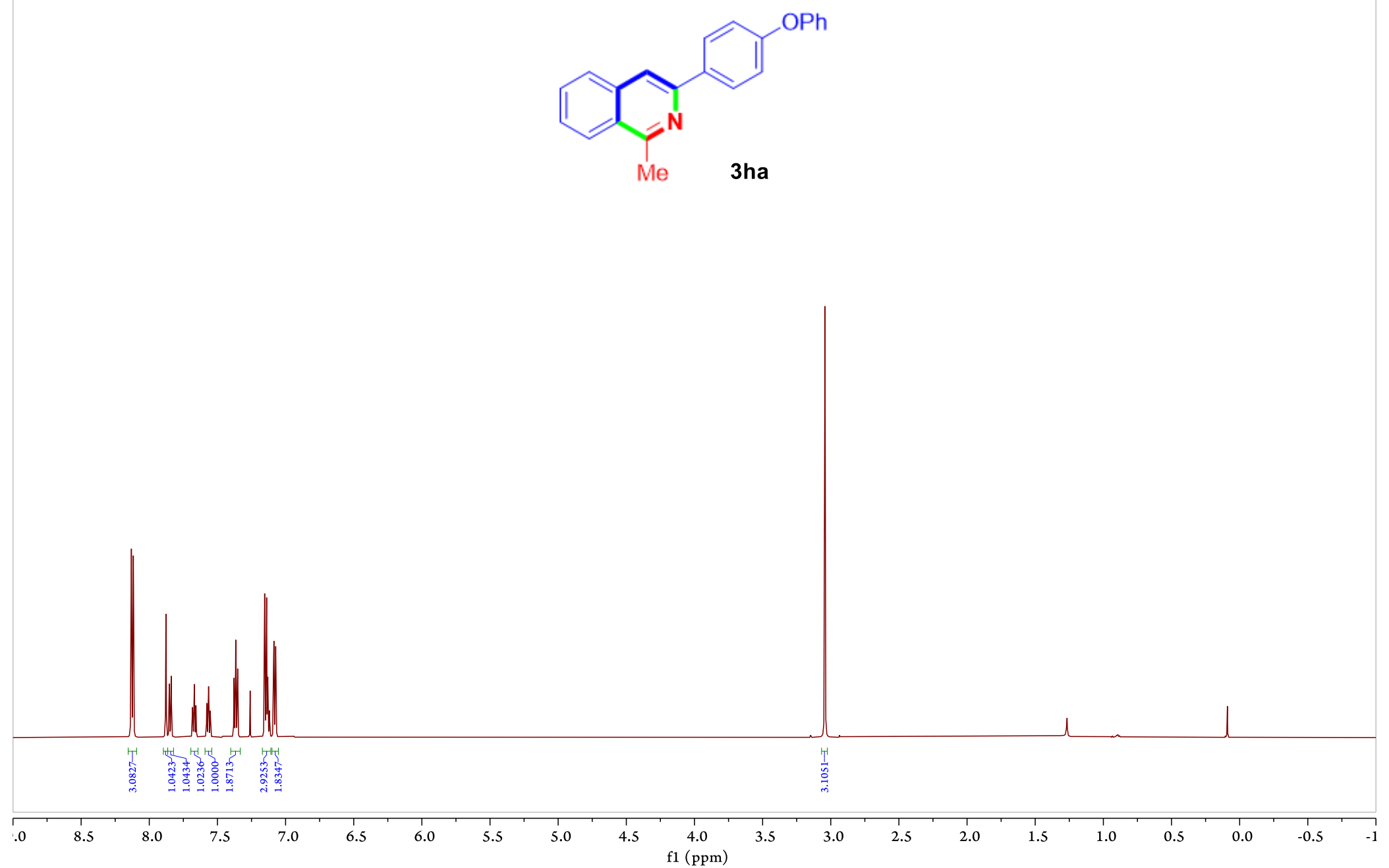


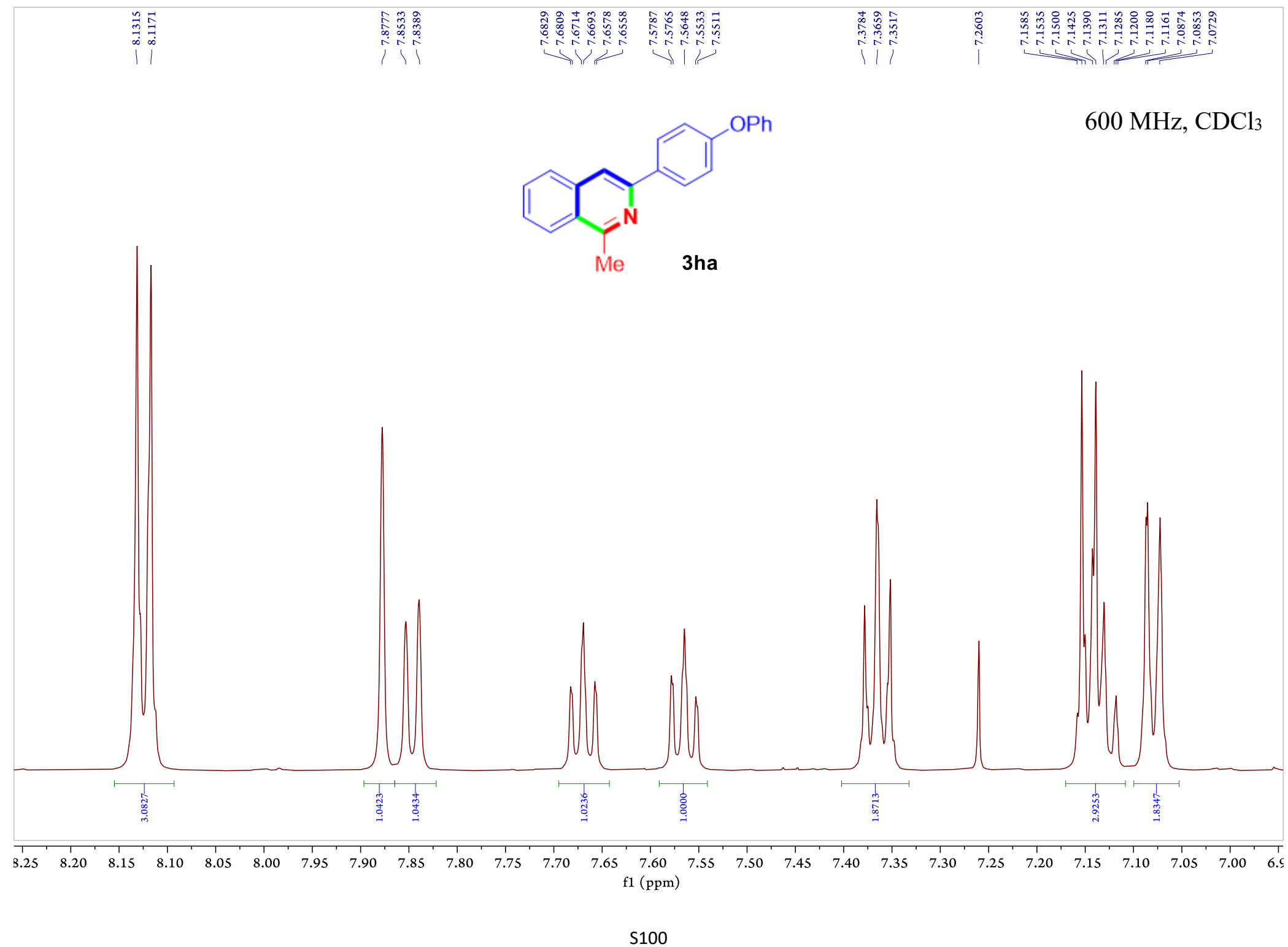




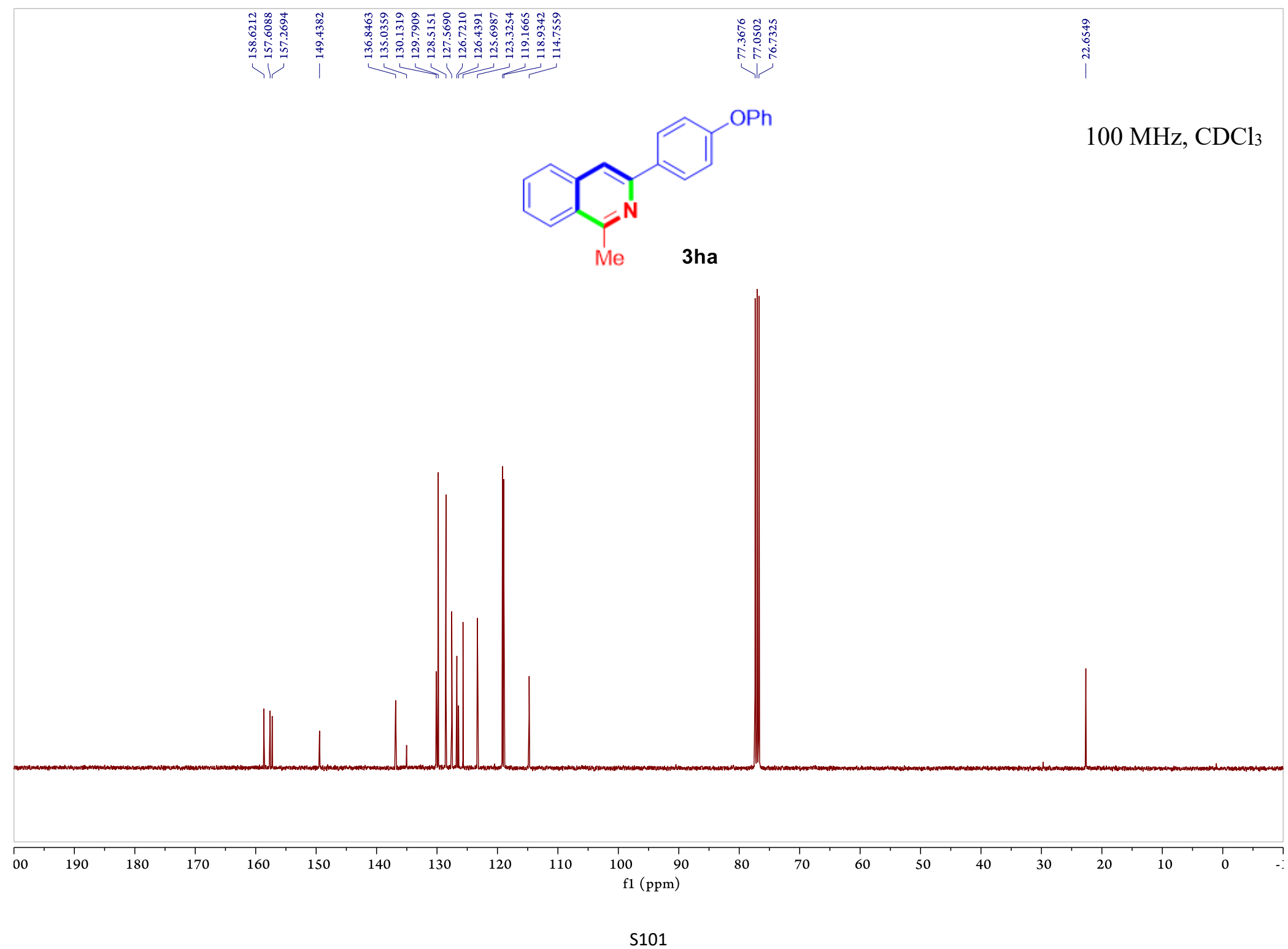




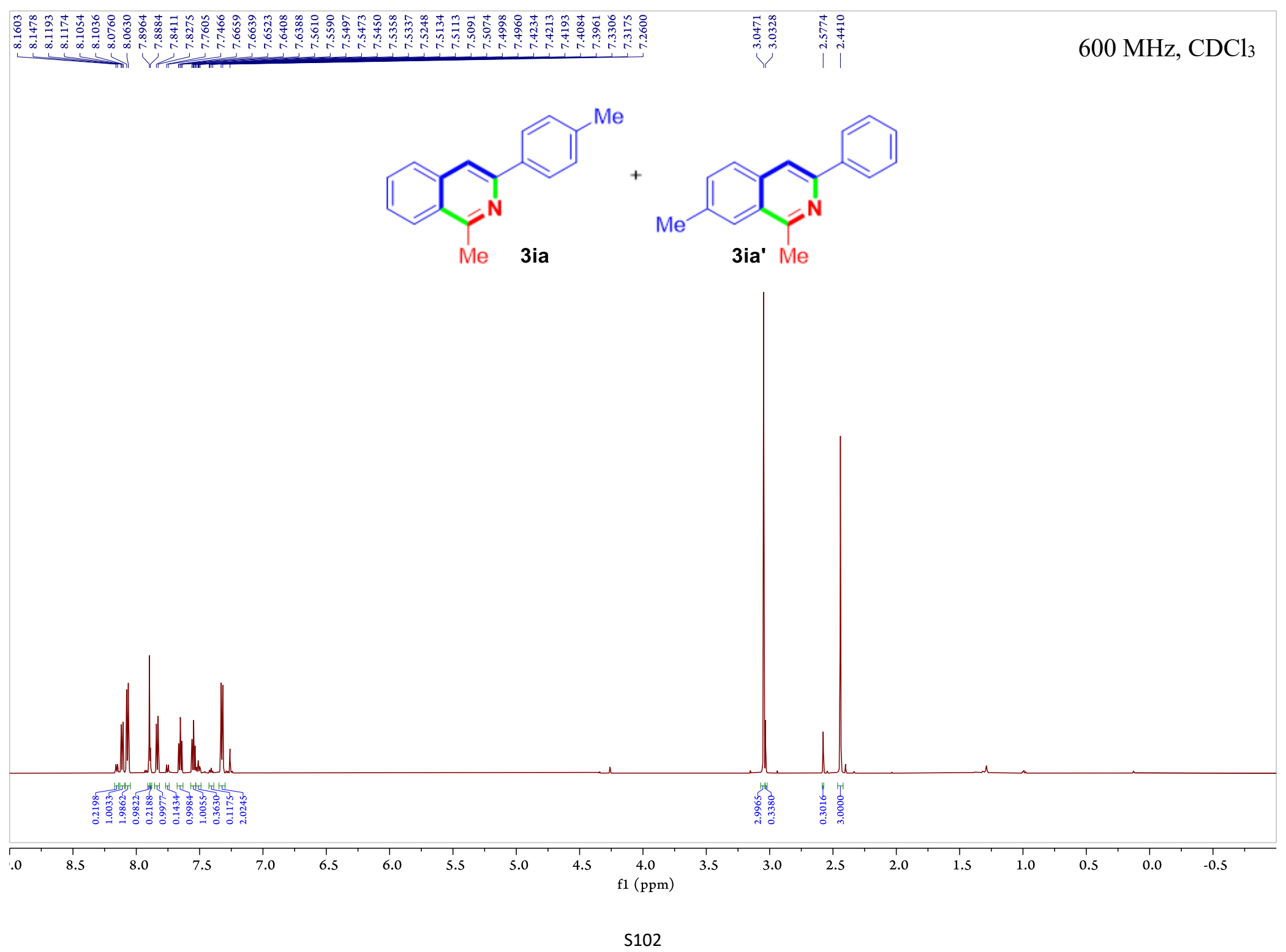




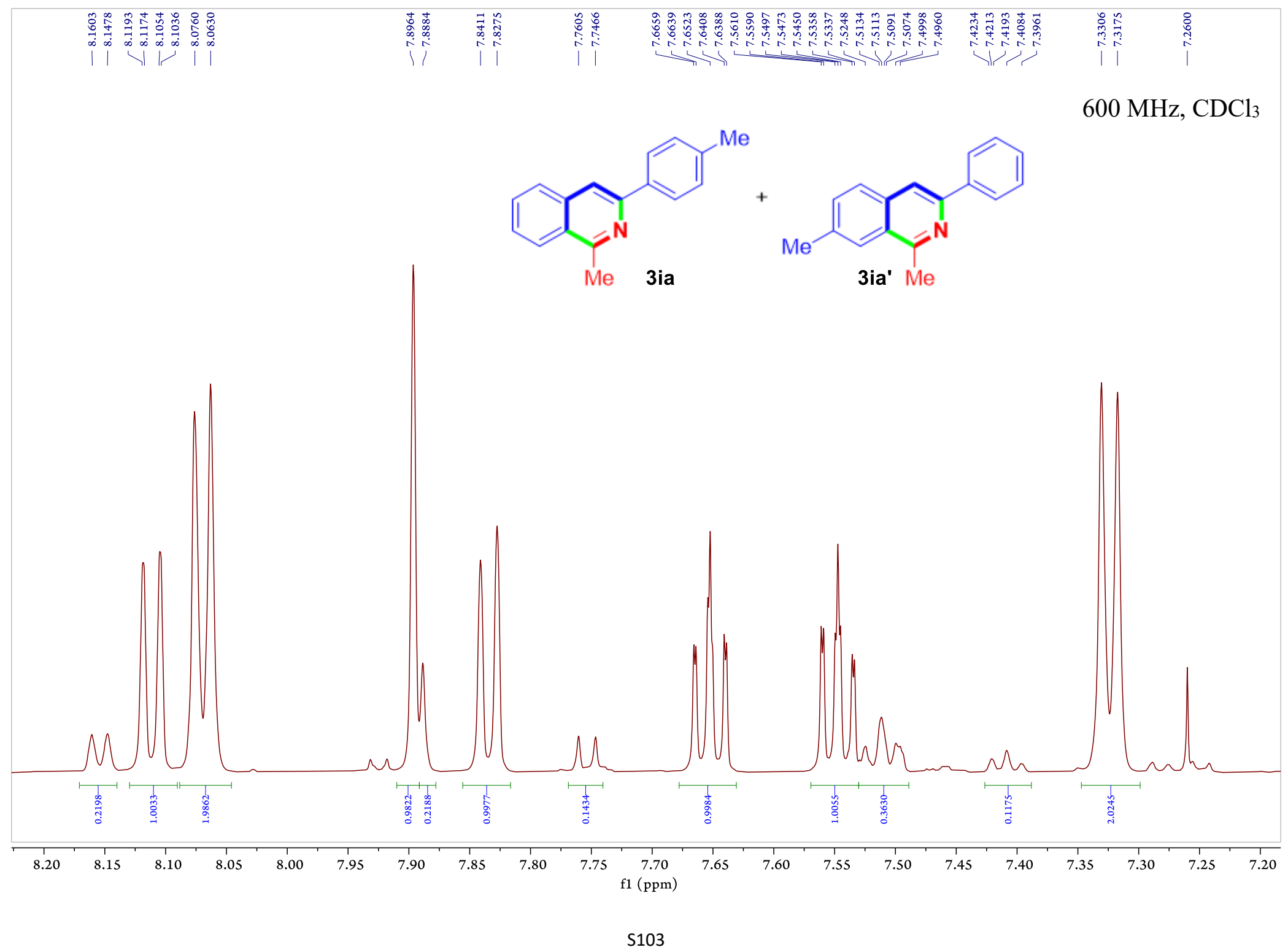




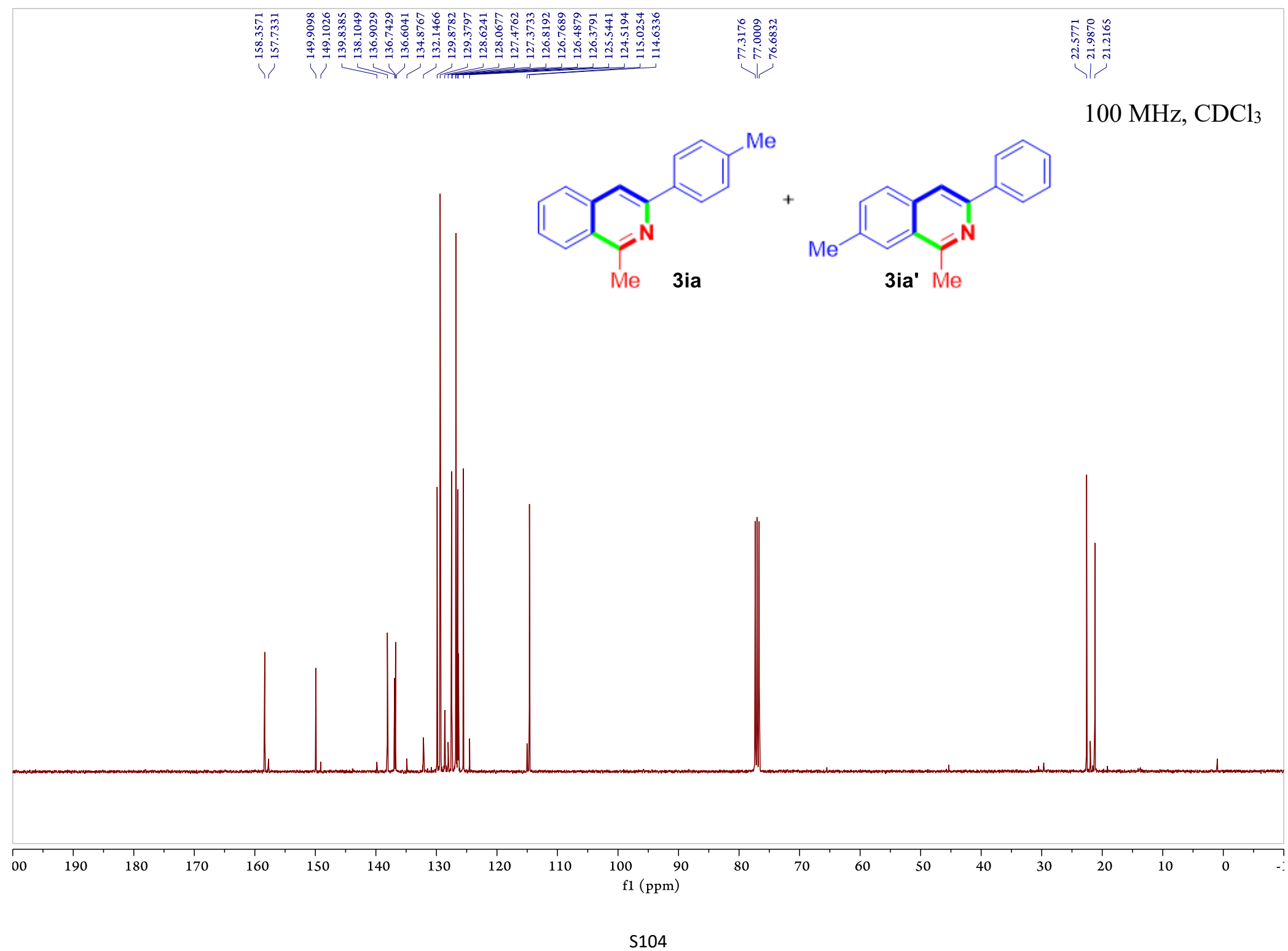




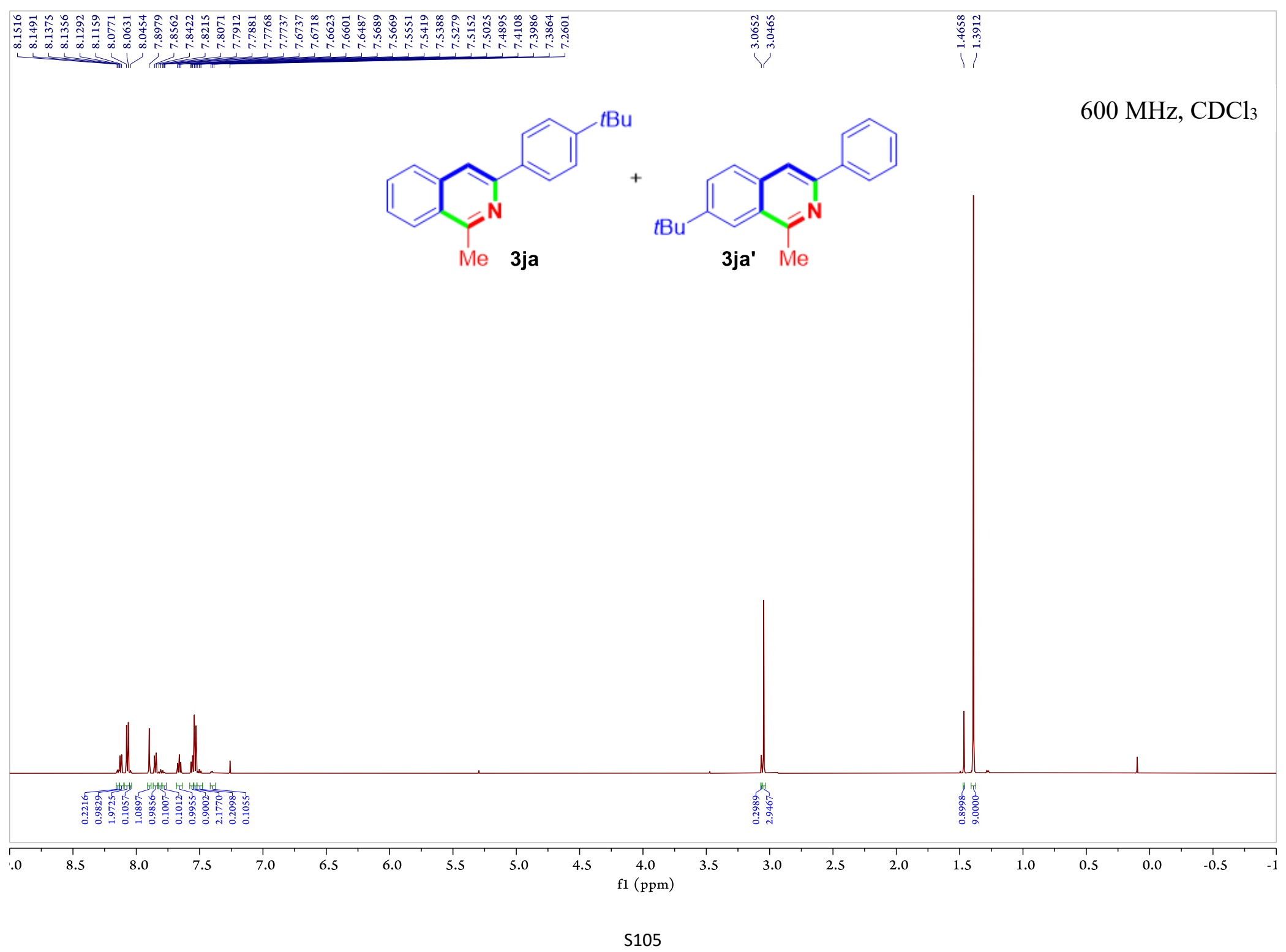




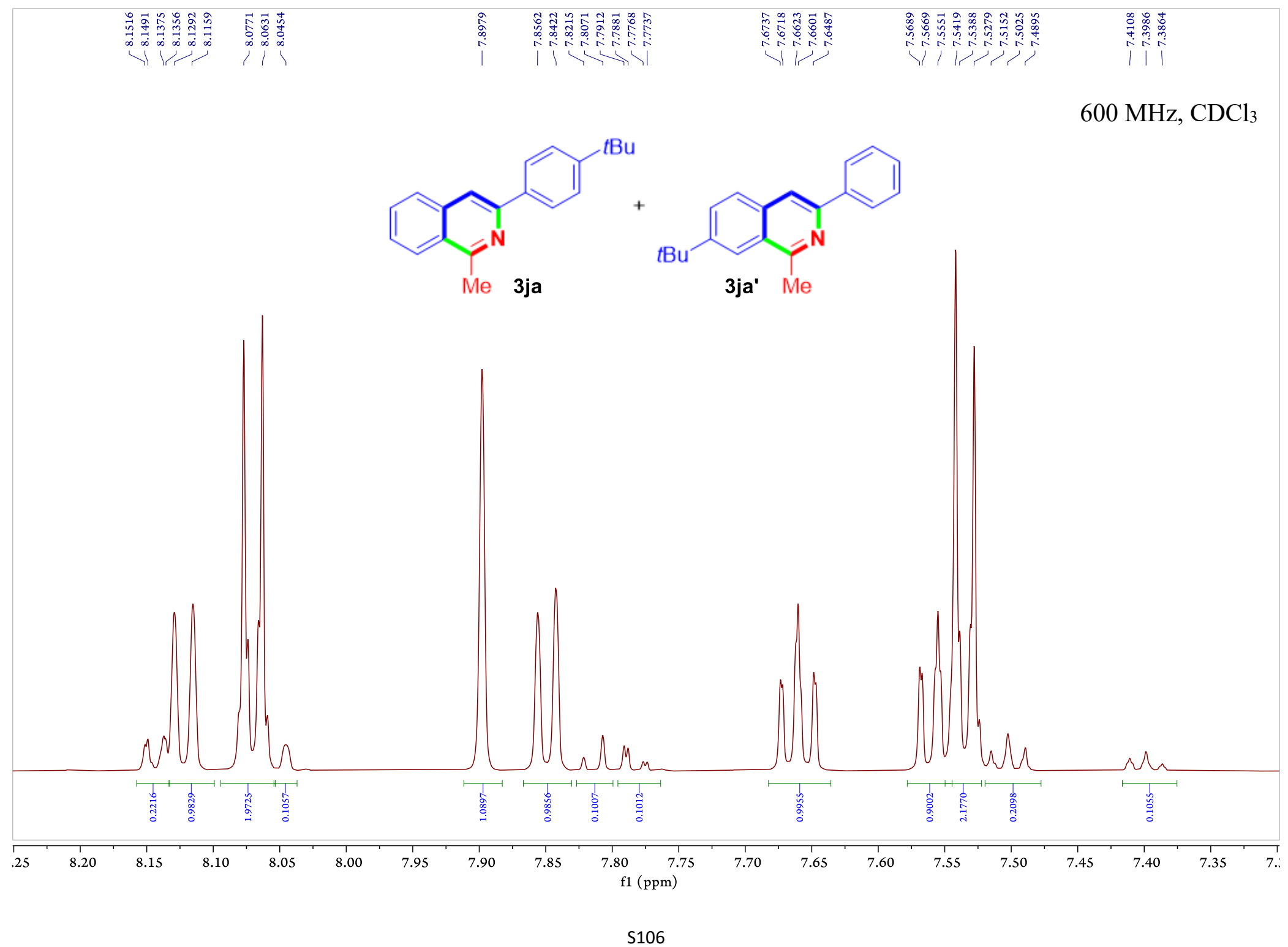




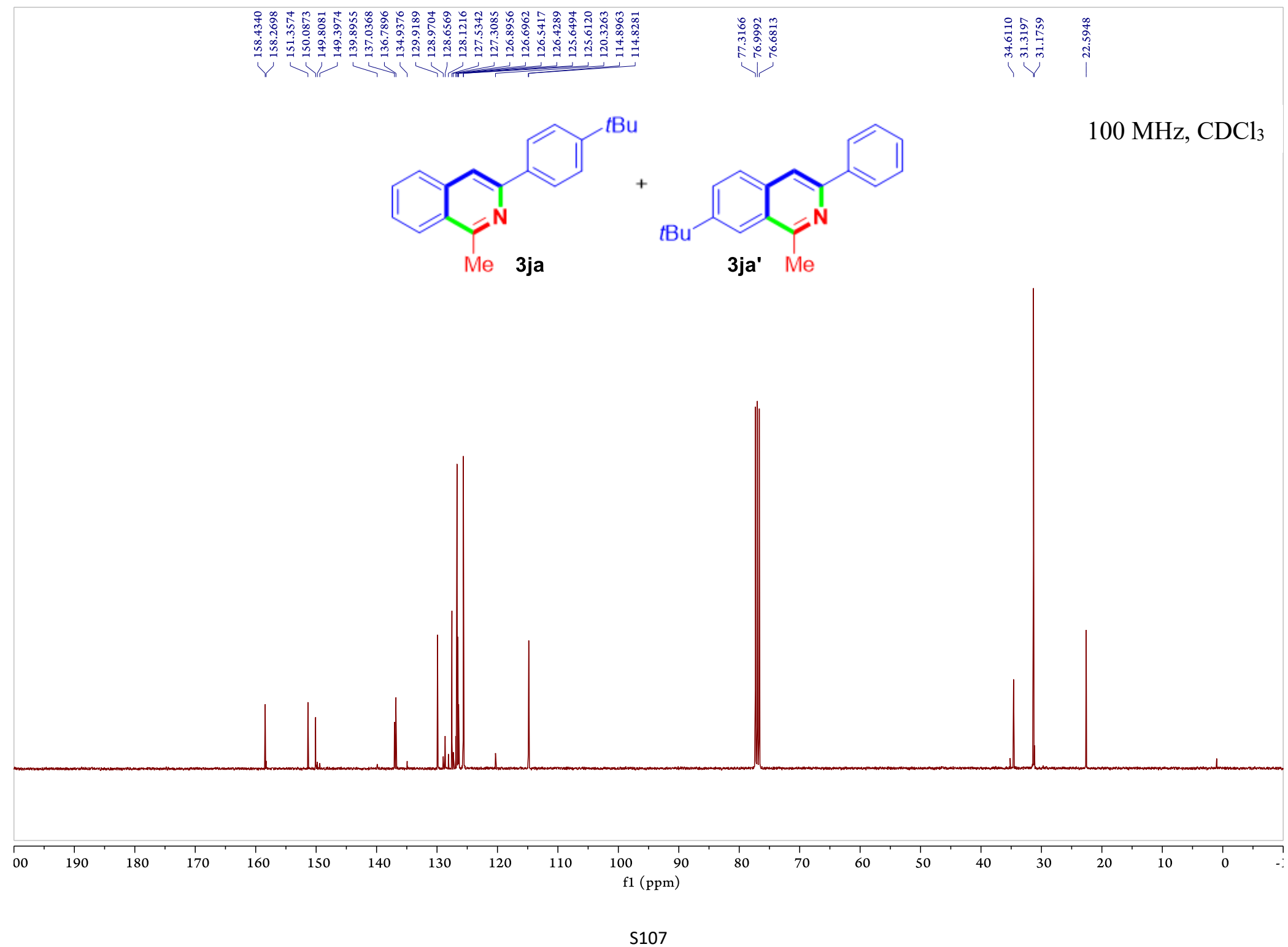




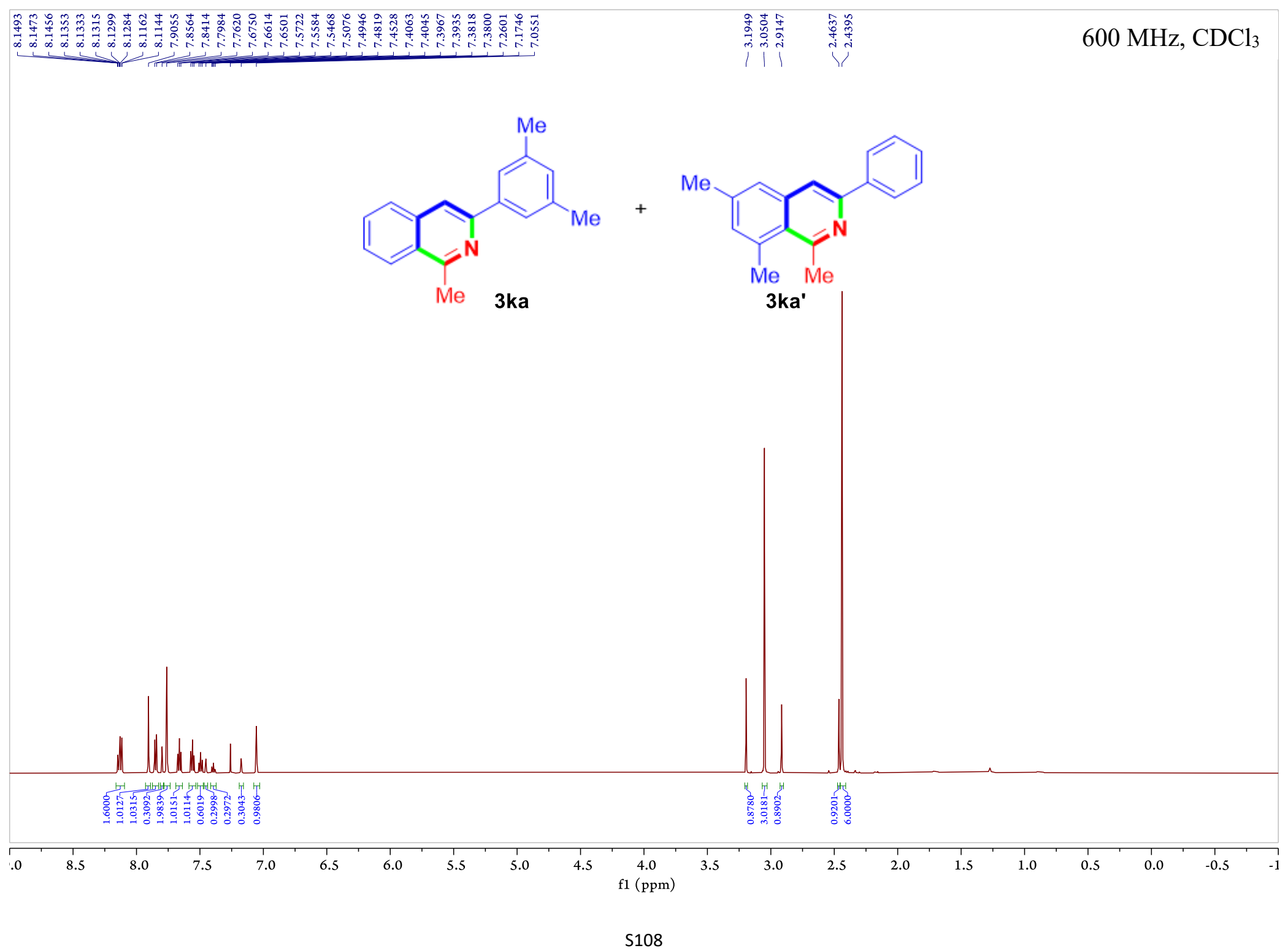




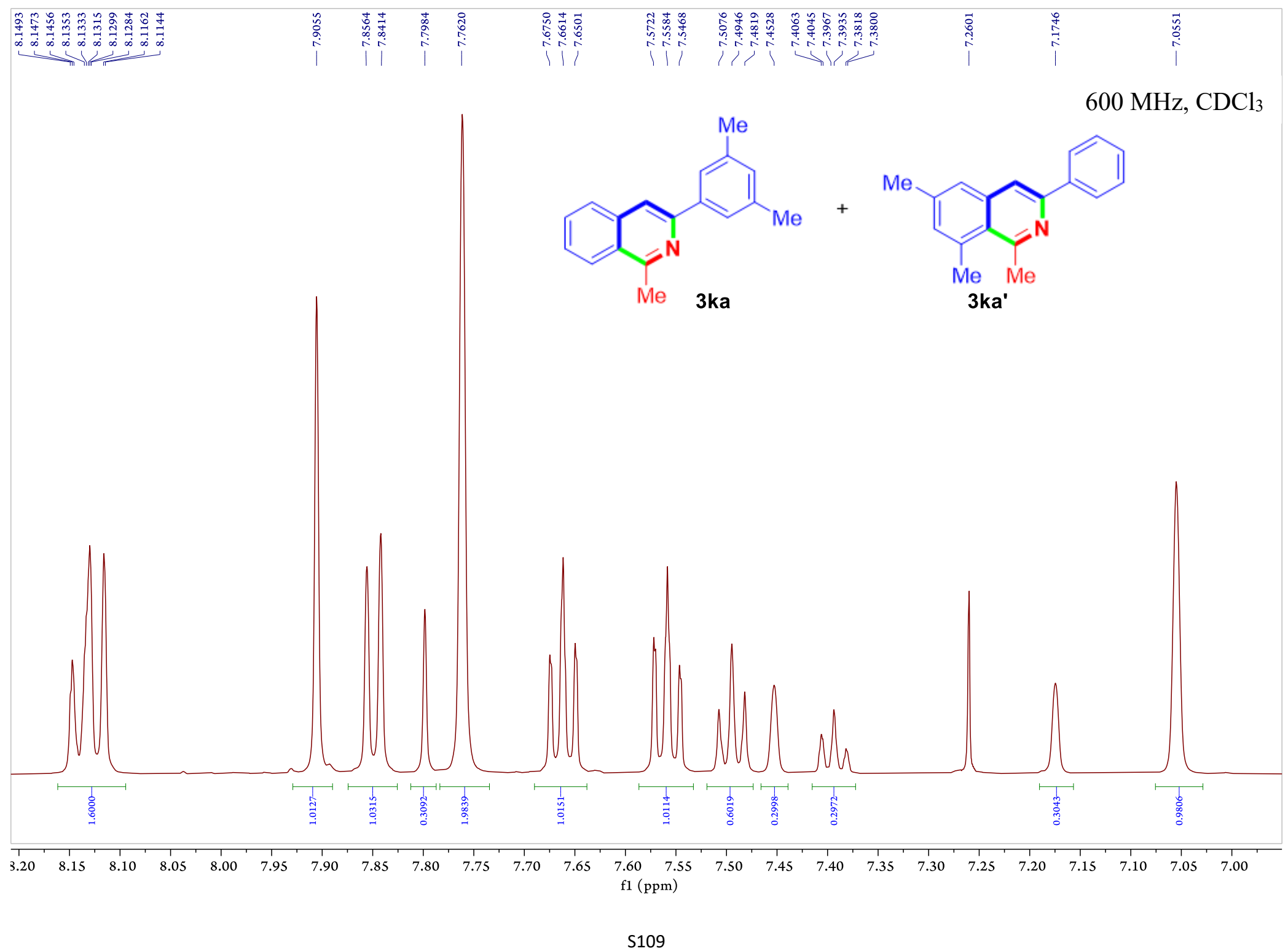




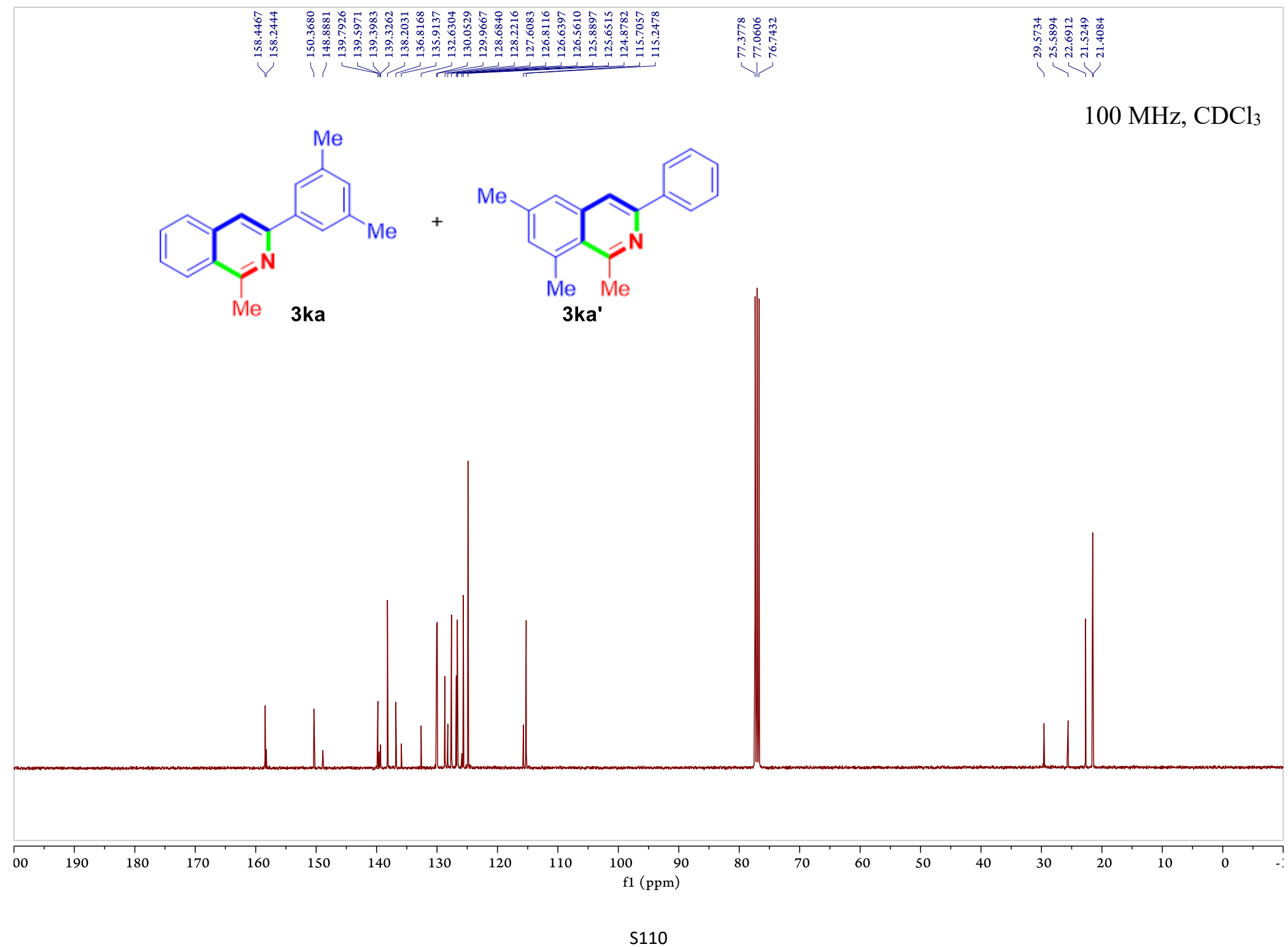




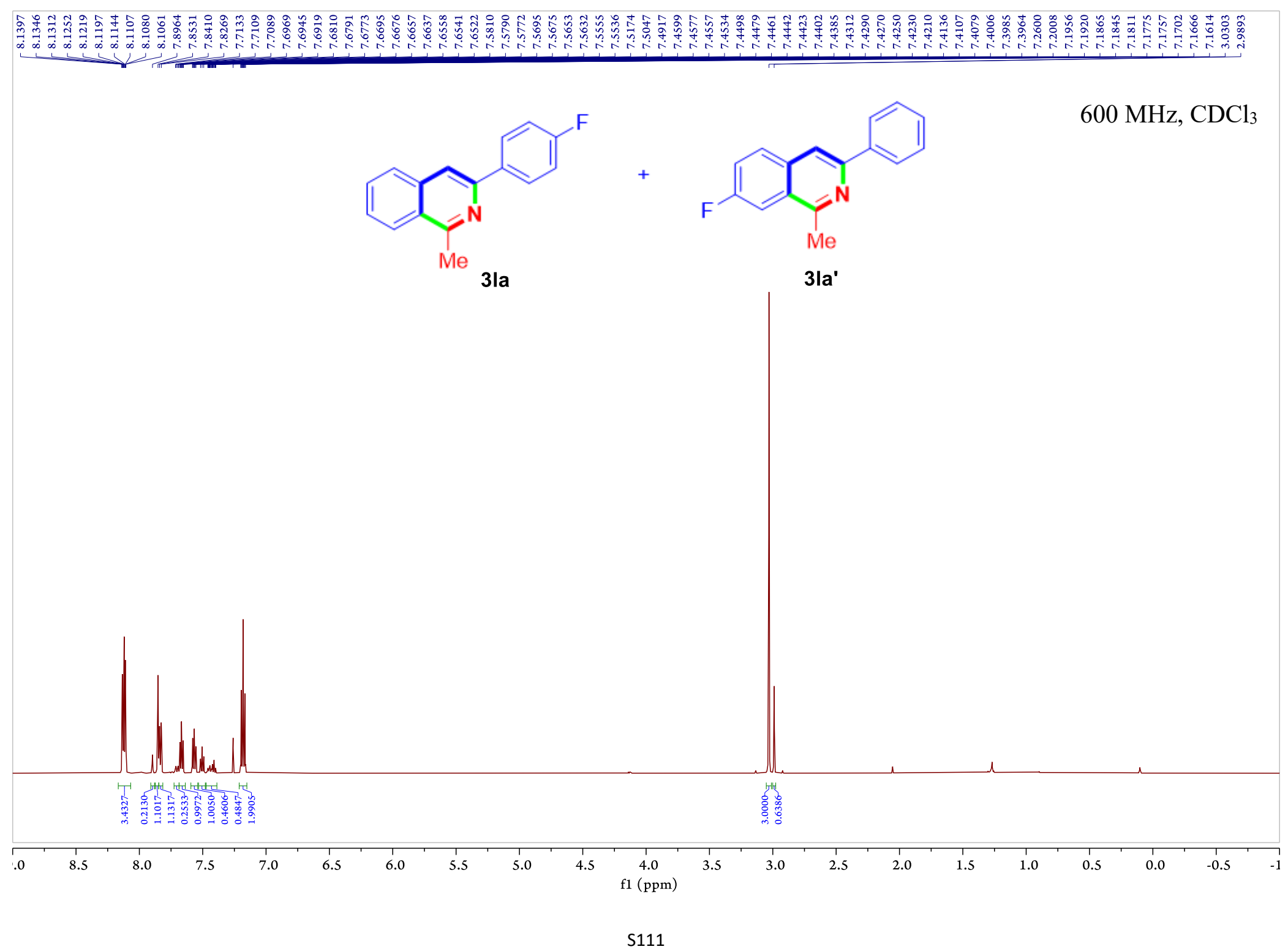




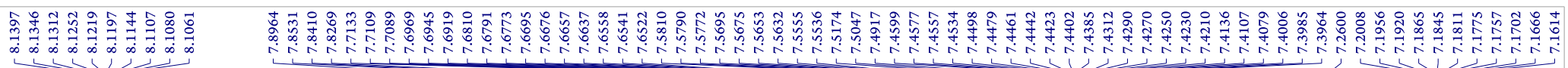

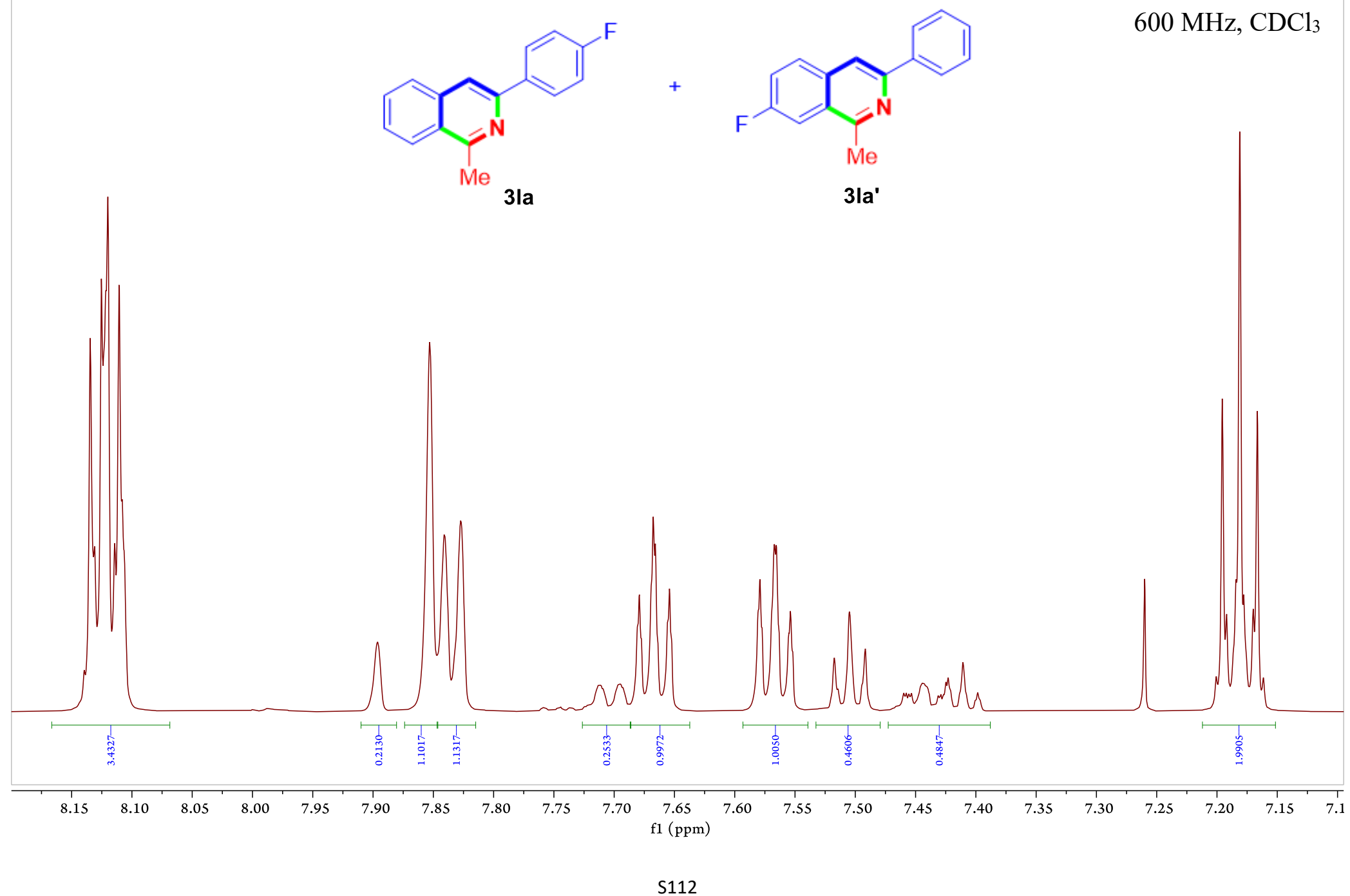




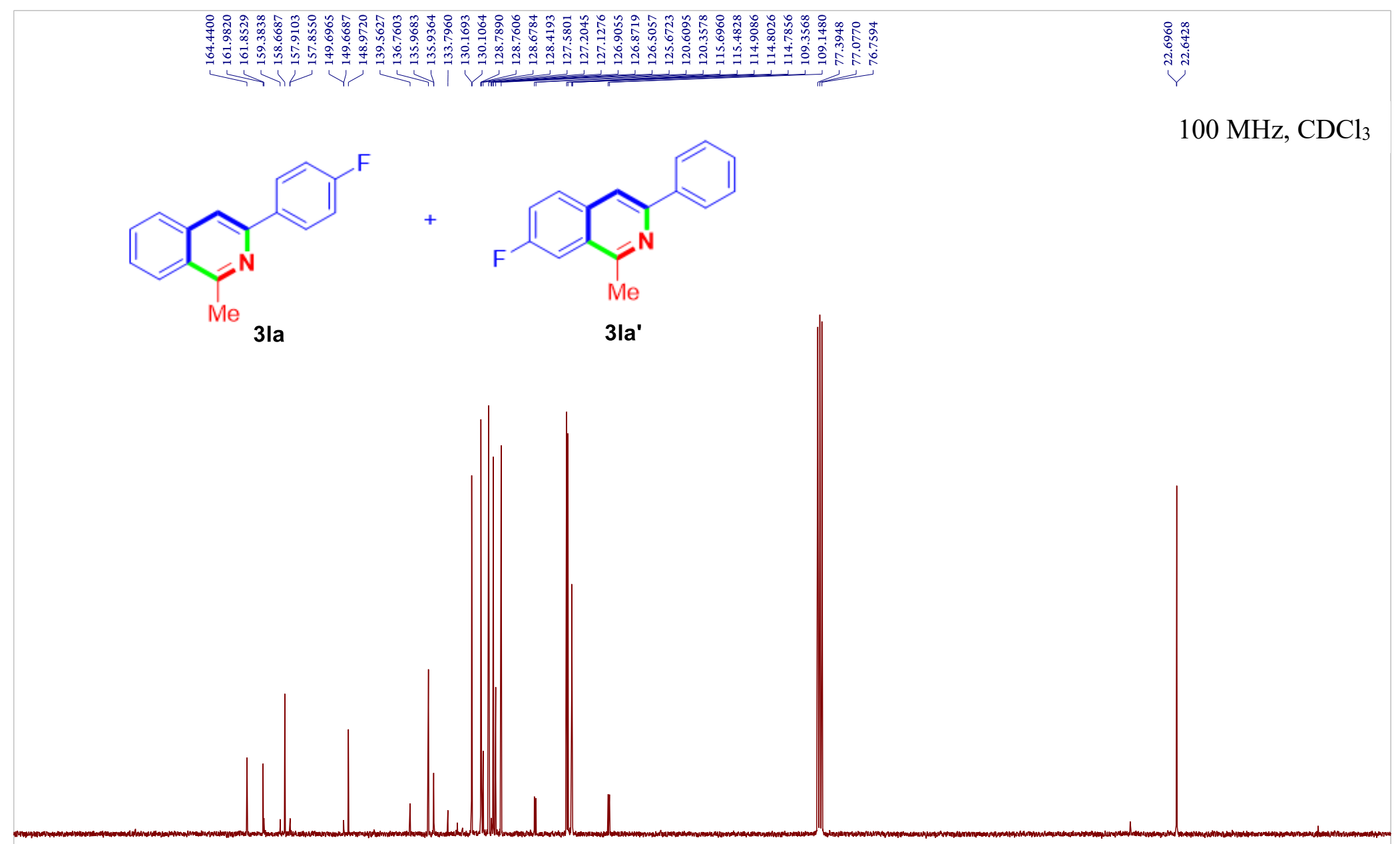

00

$190 \quad 180$

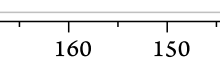

40
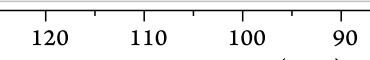

f1 (ppm) 


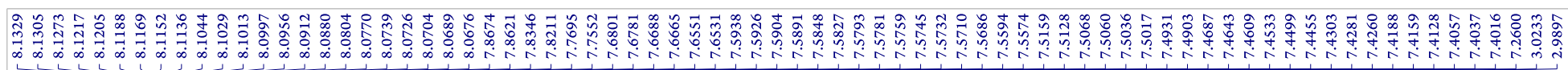

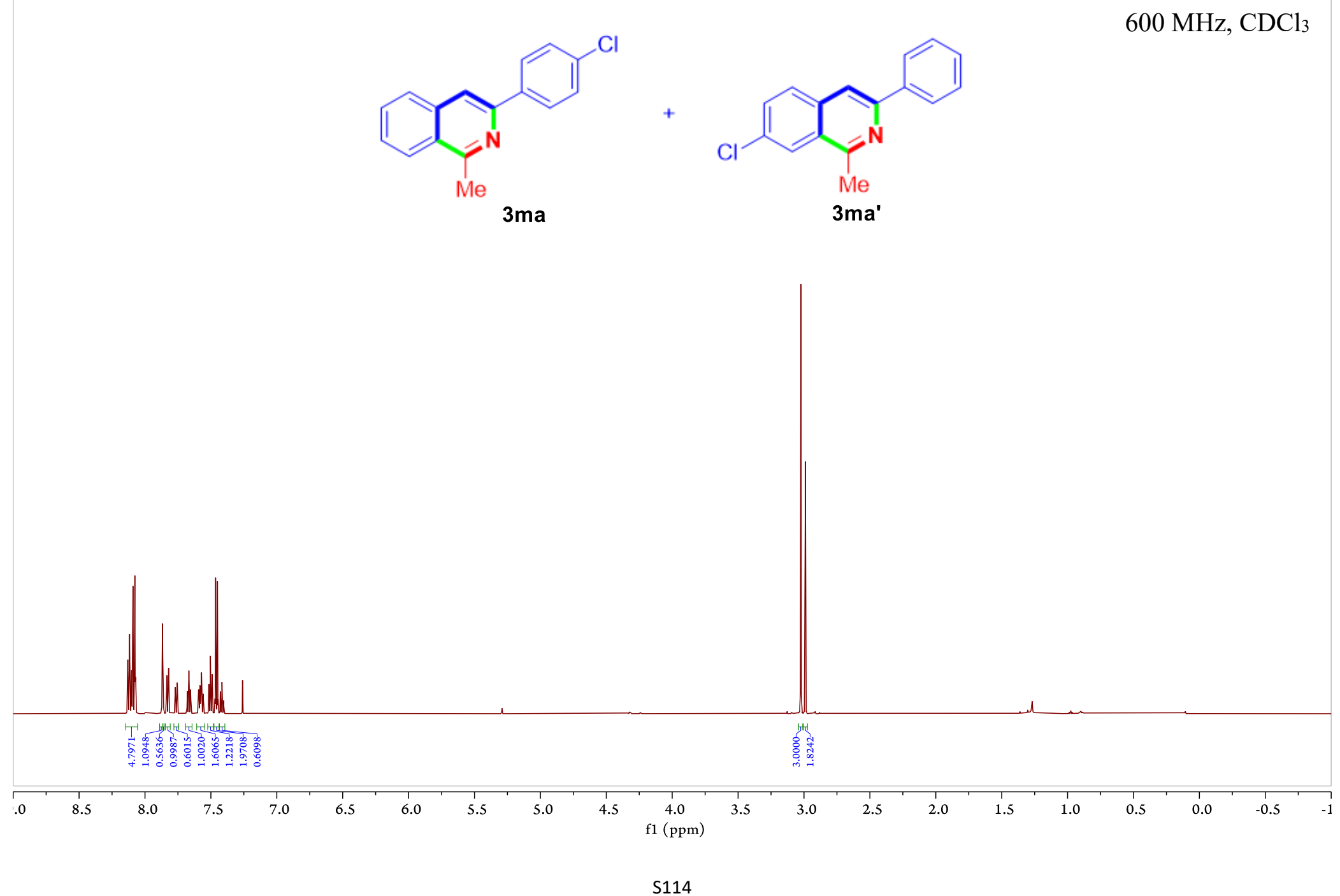




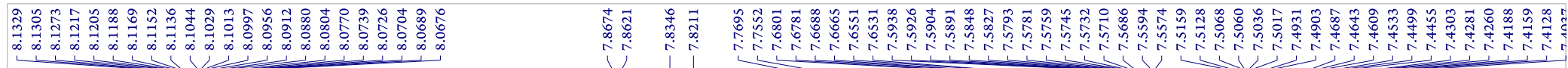

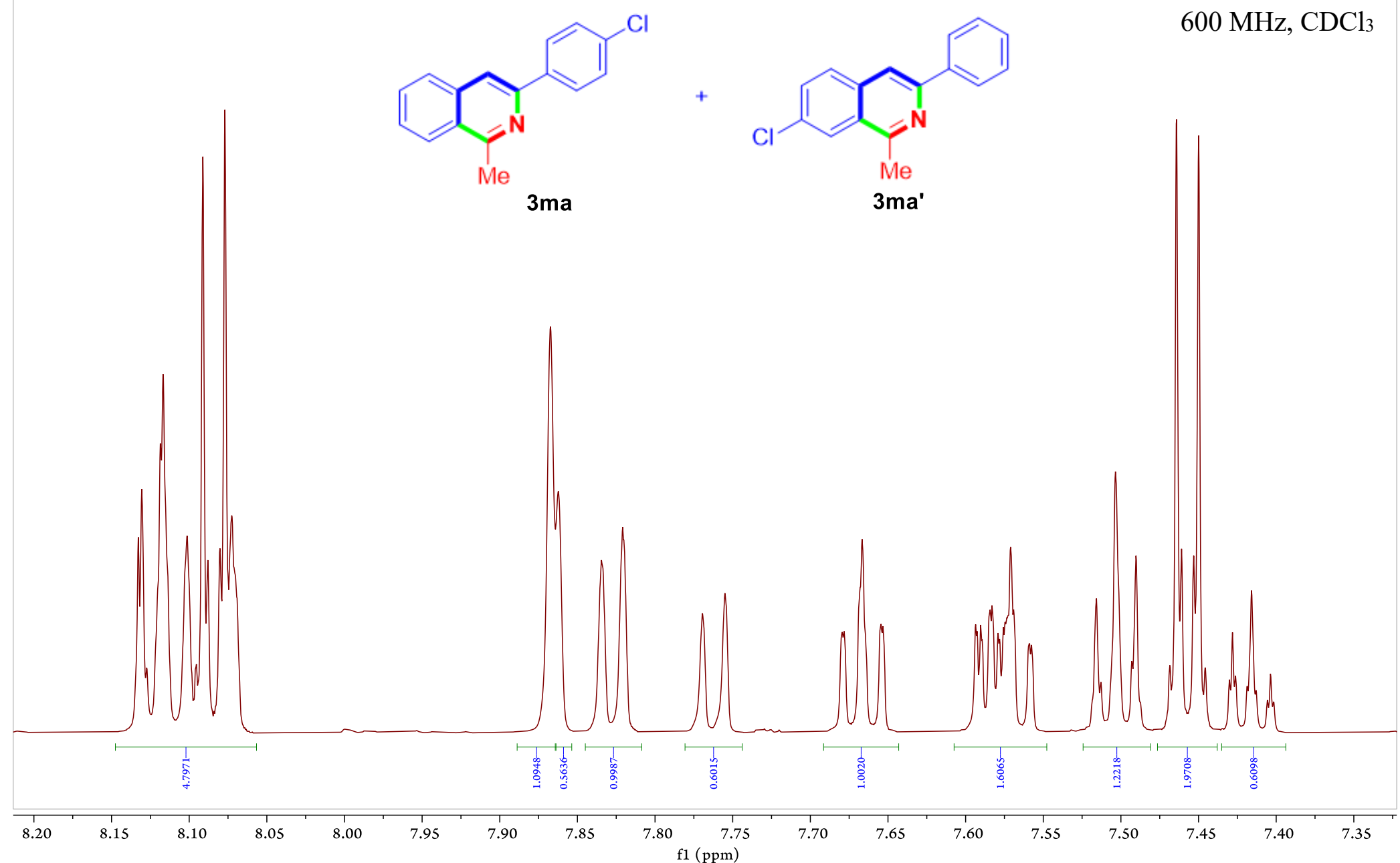




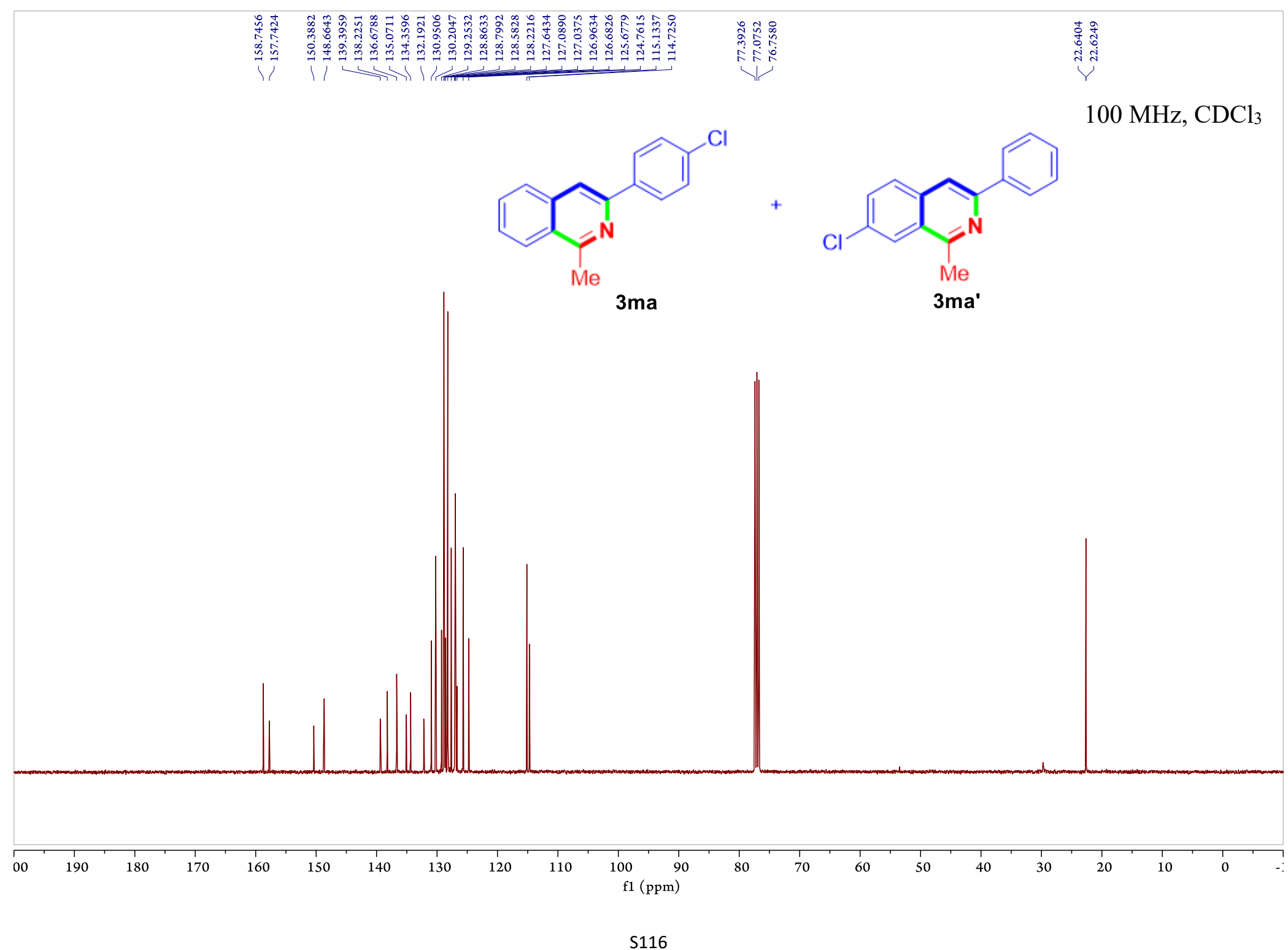




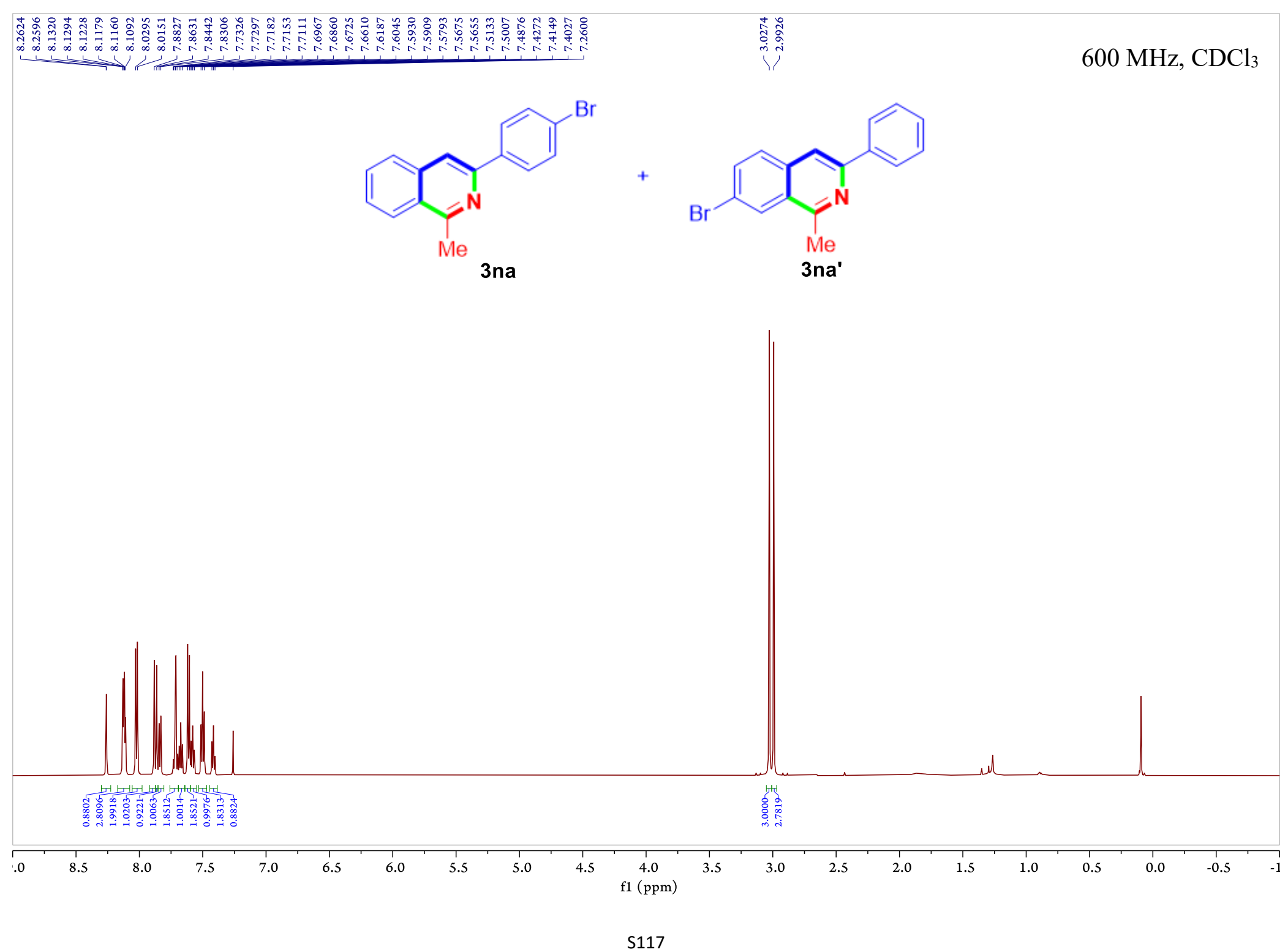




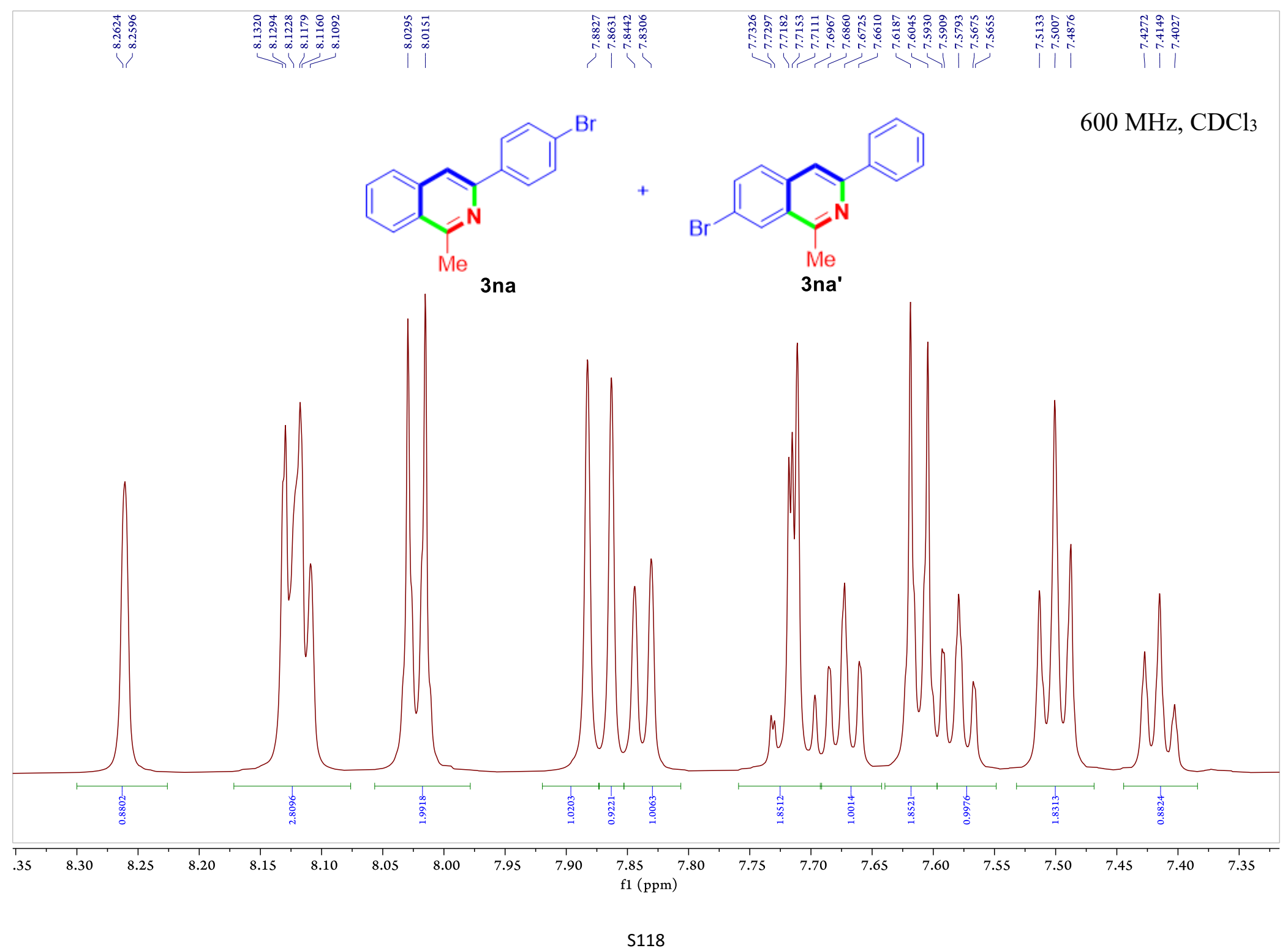




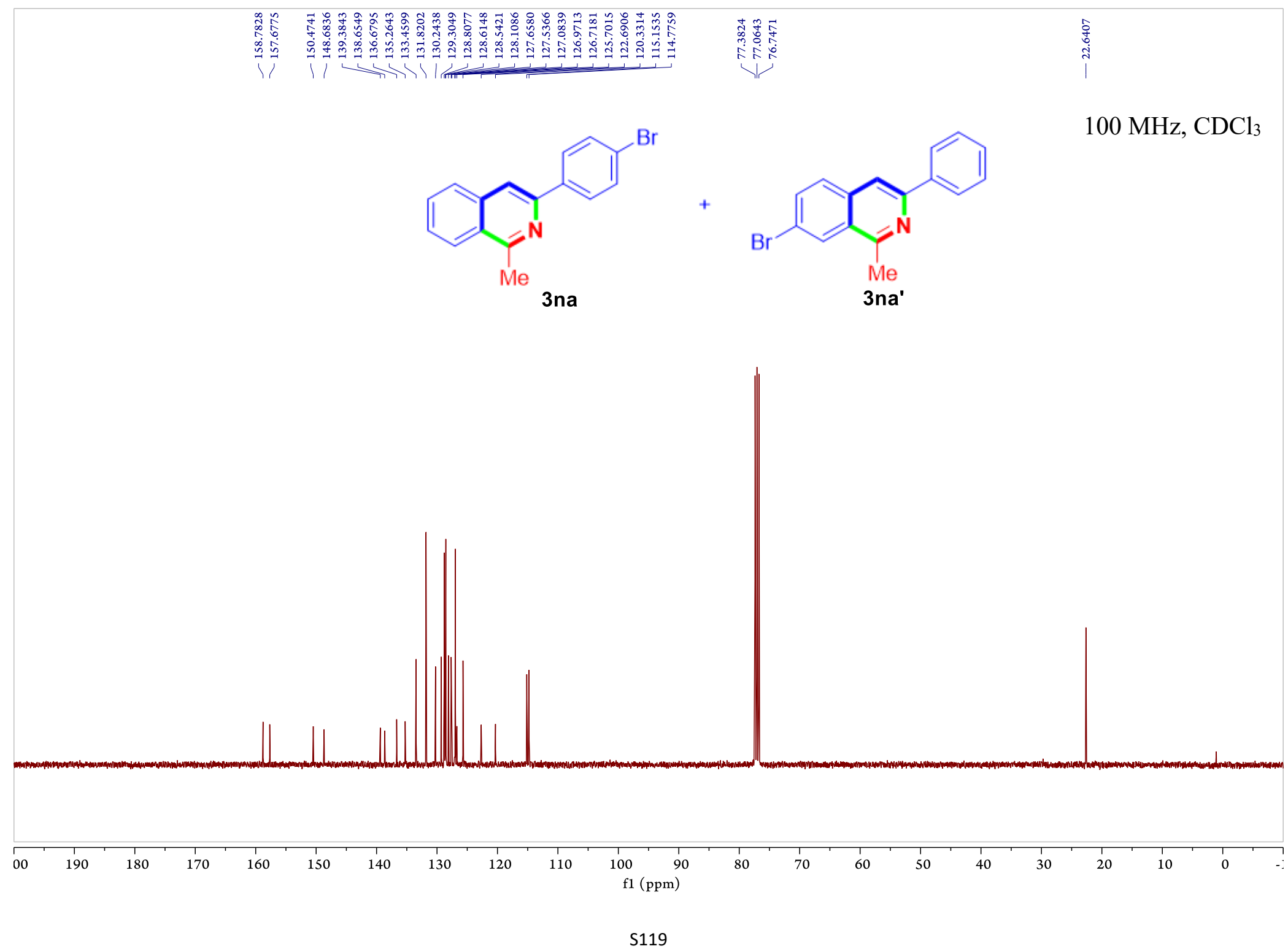




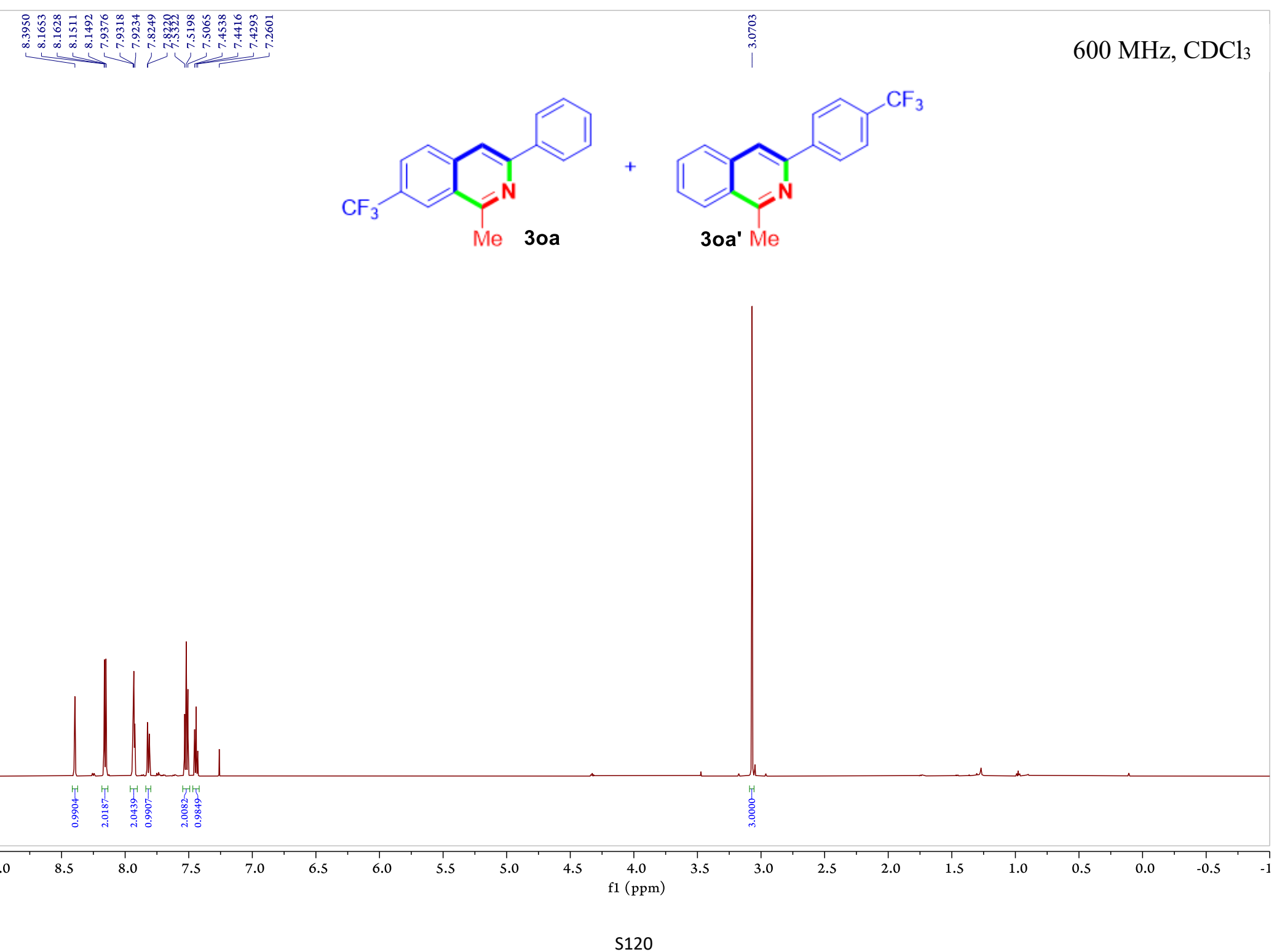




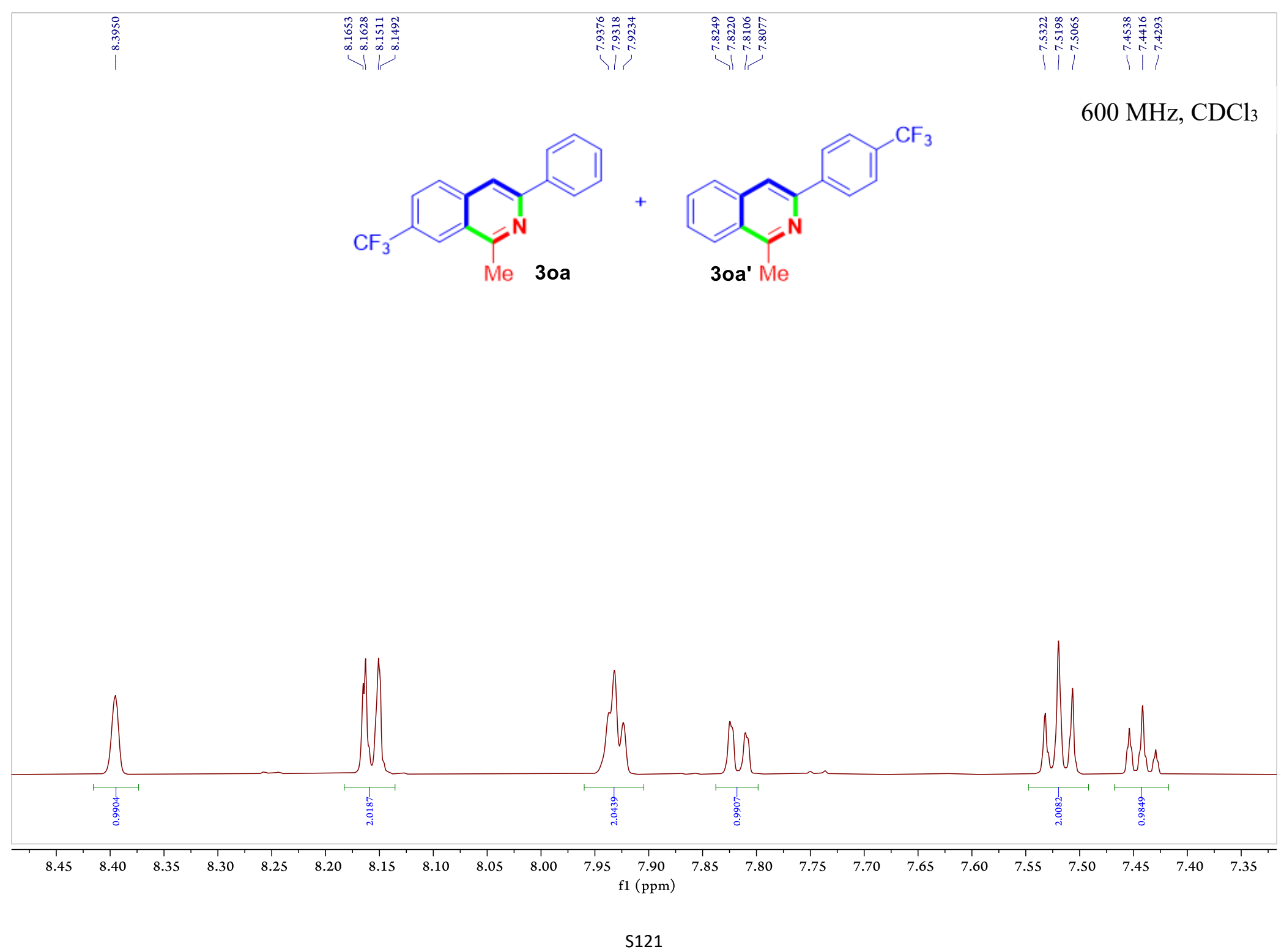




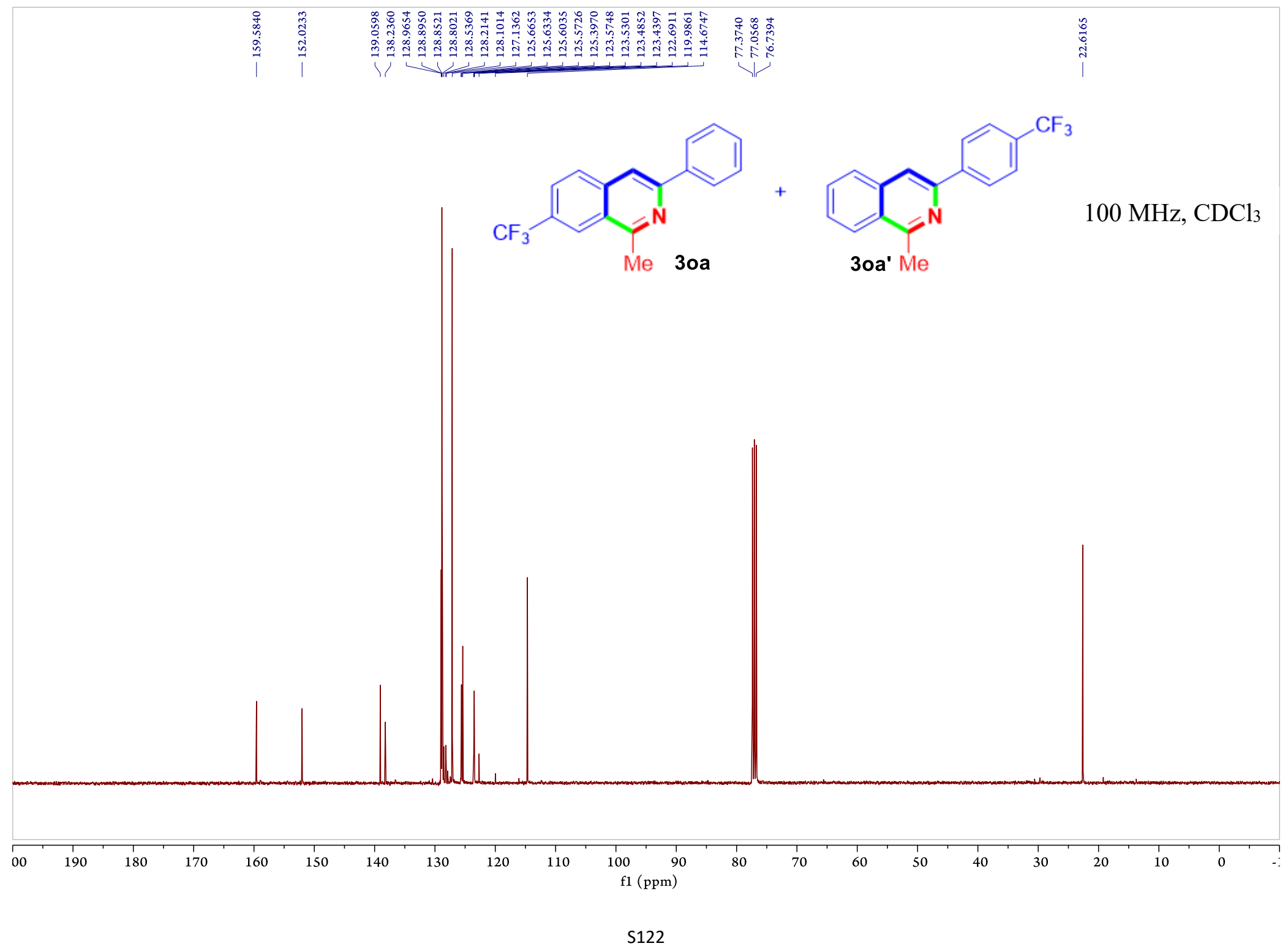




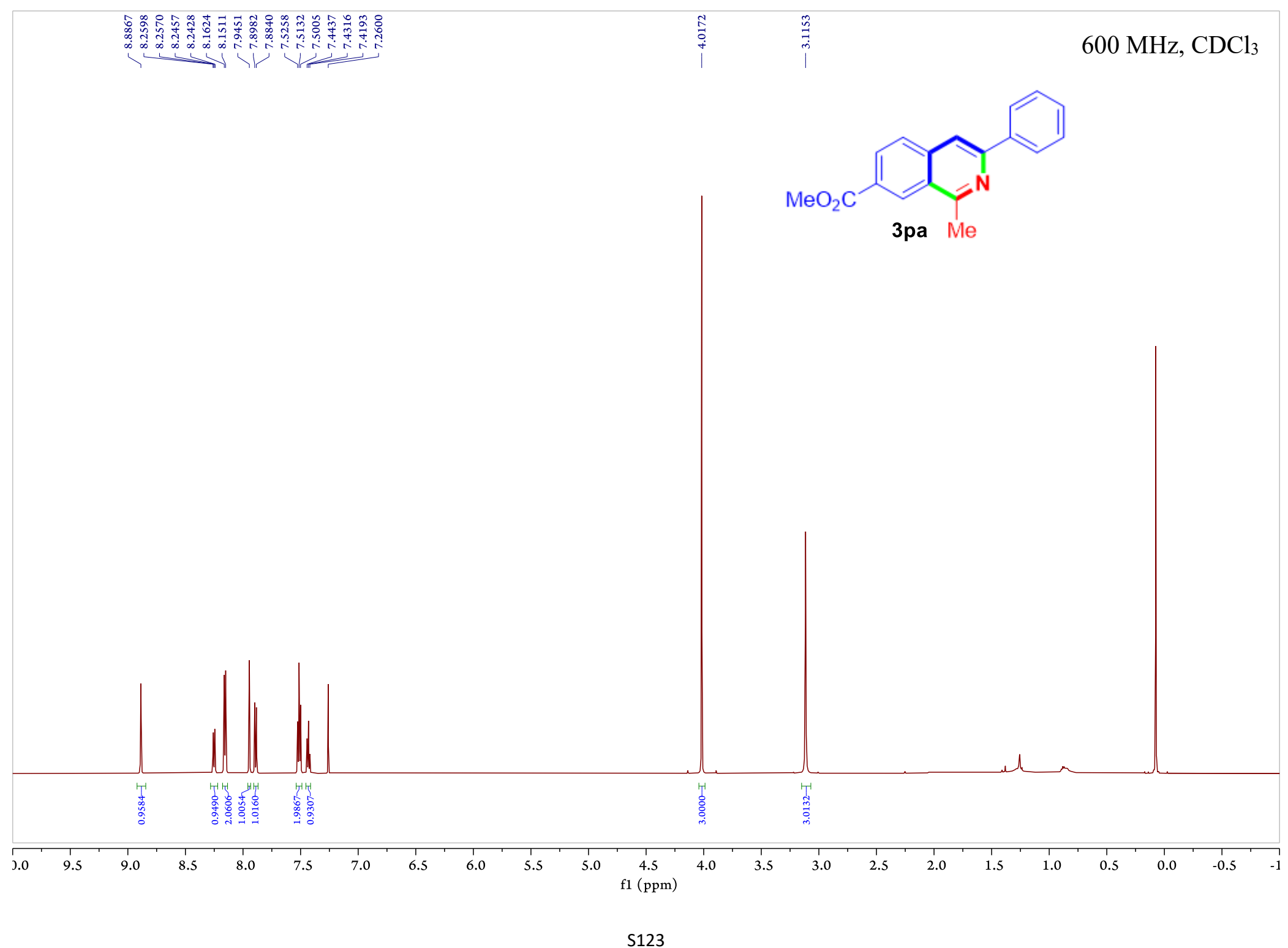




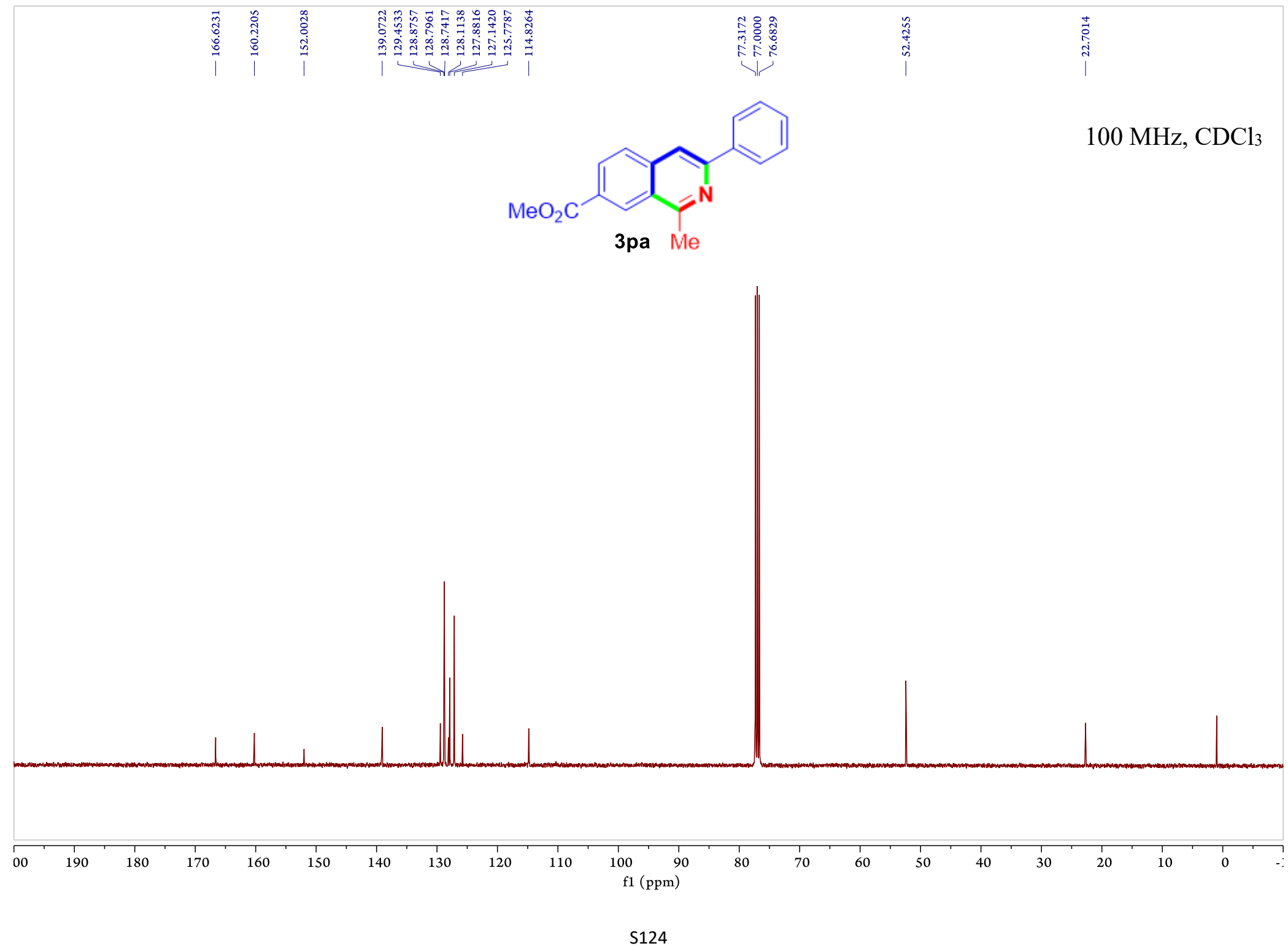




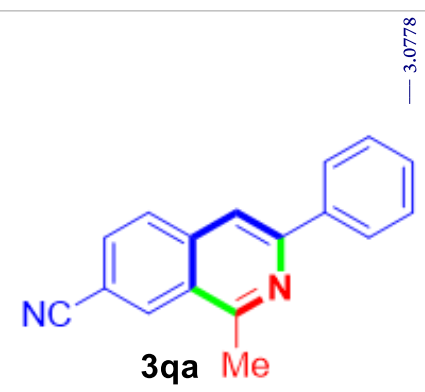

$600 \mathrm{MHz}, \mathrm{CDCl}_{3}$

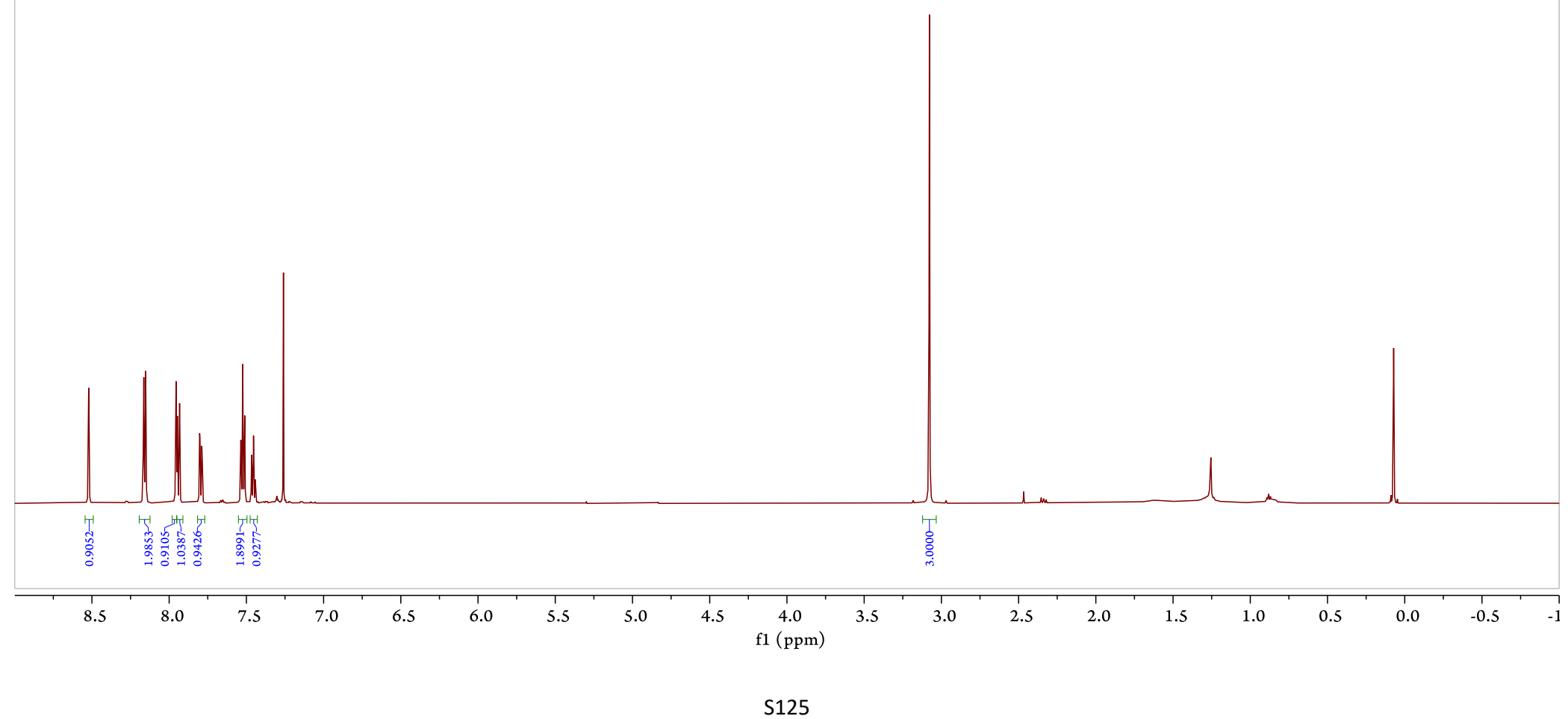




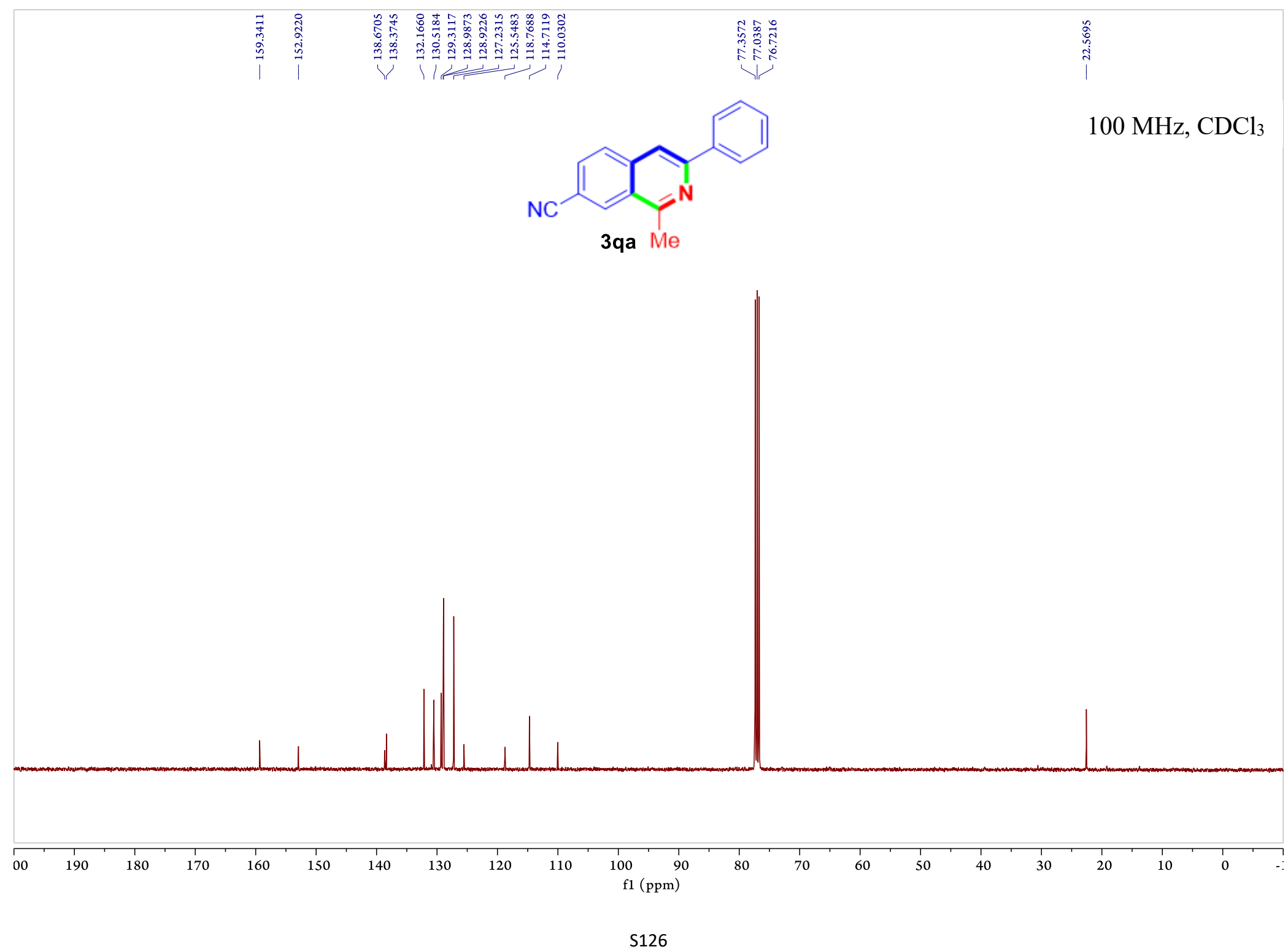




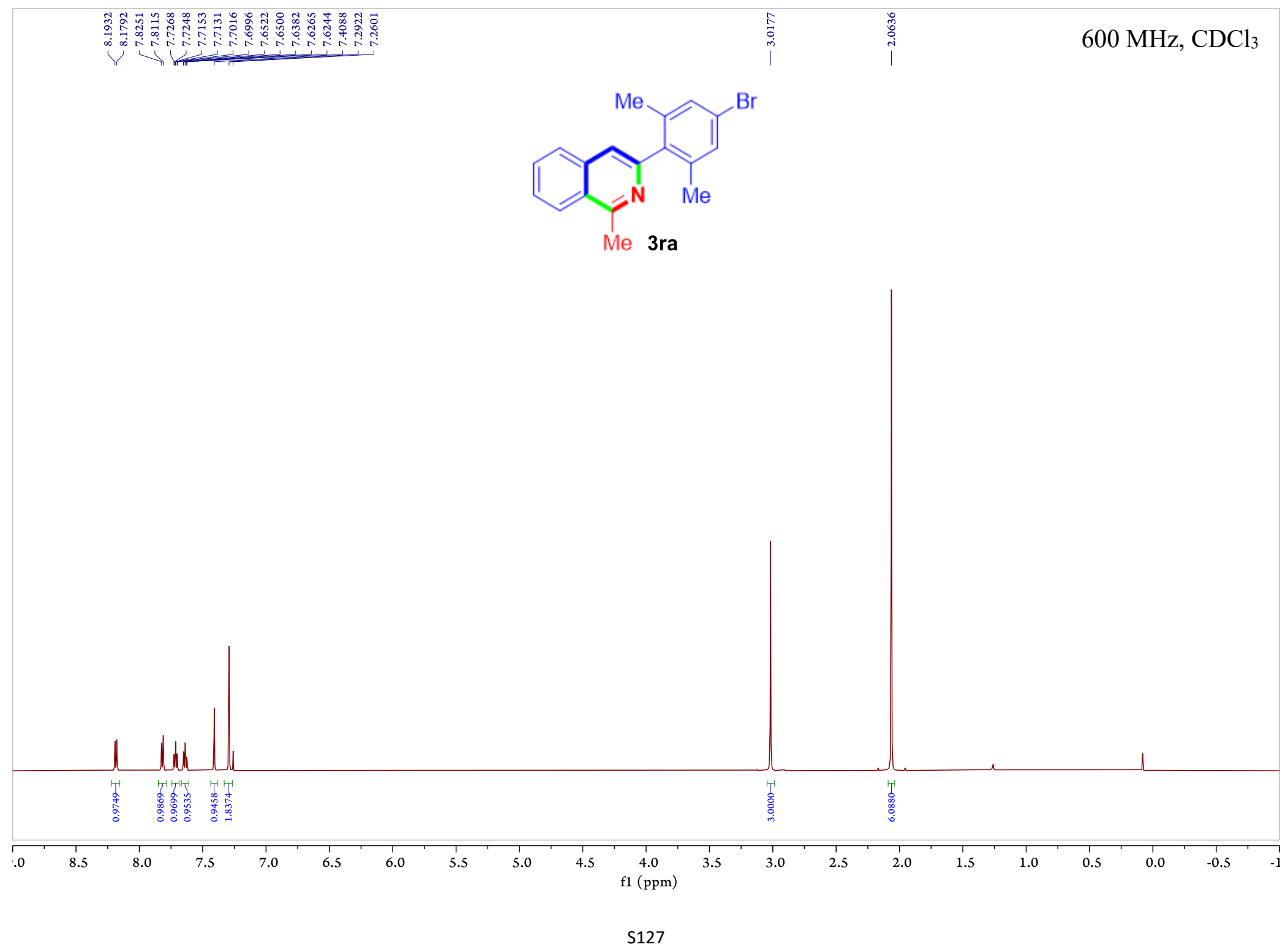




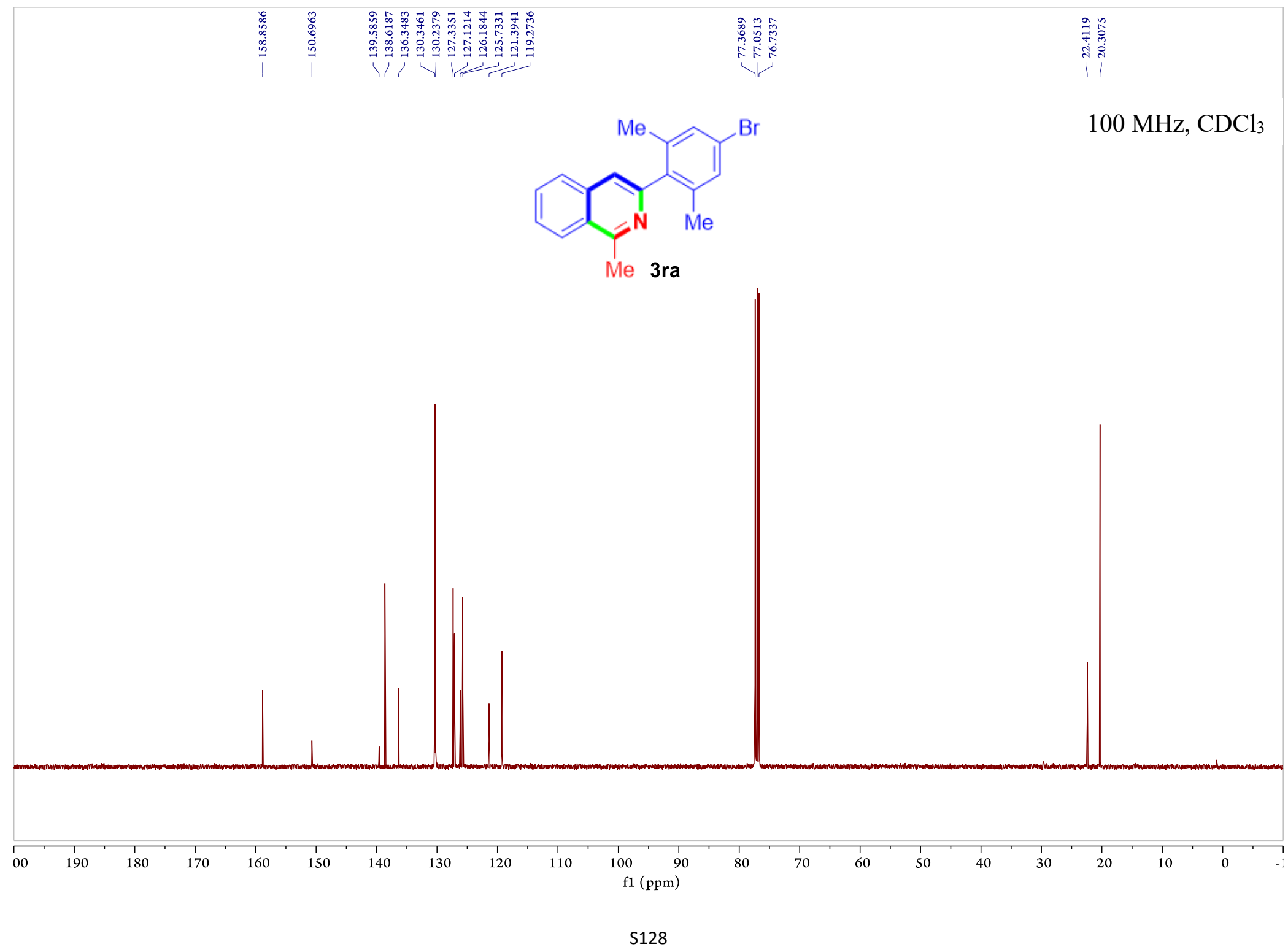




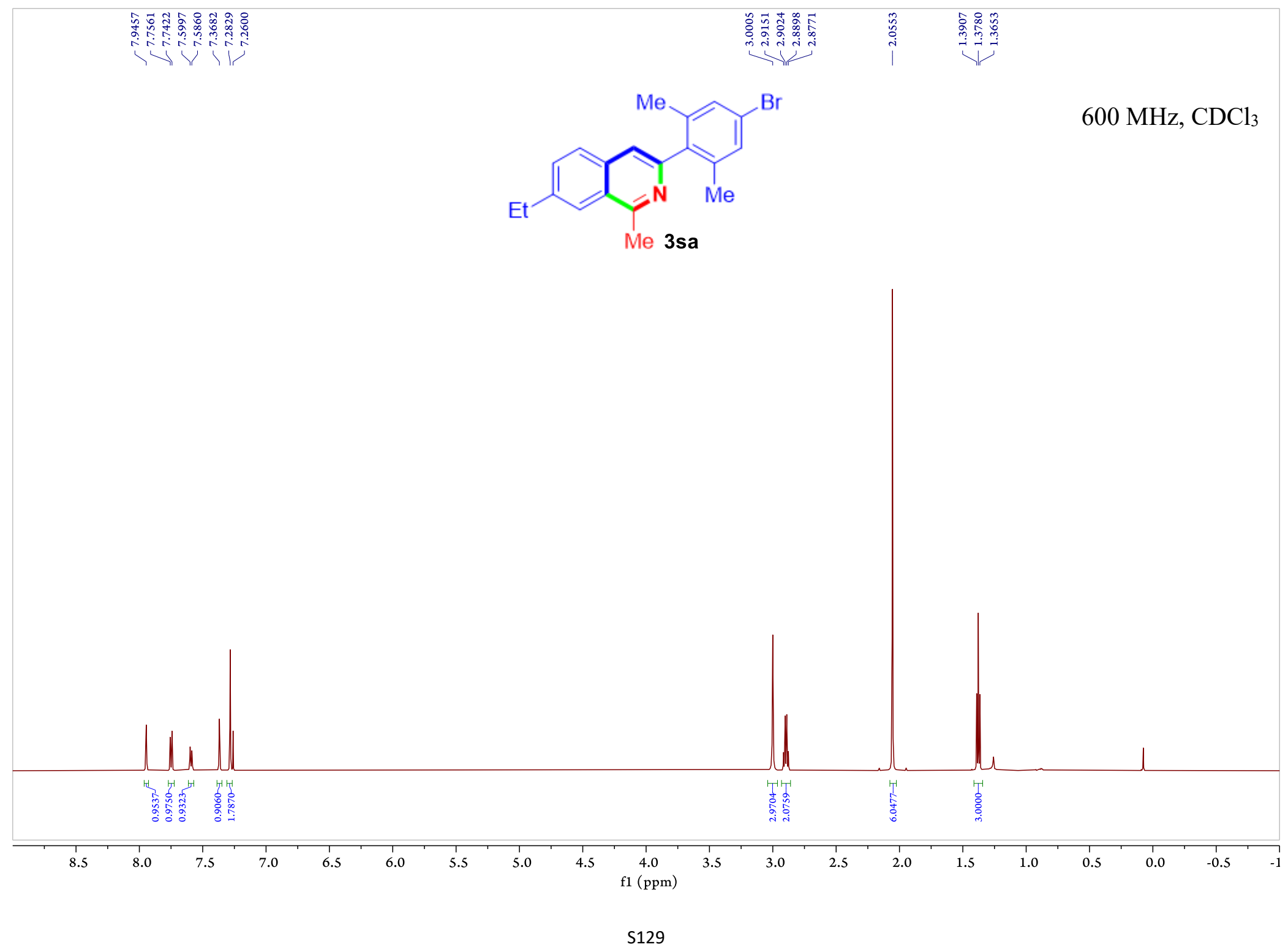




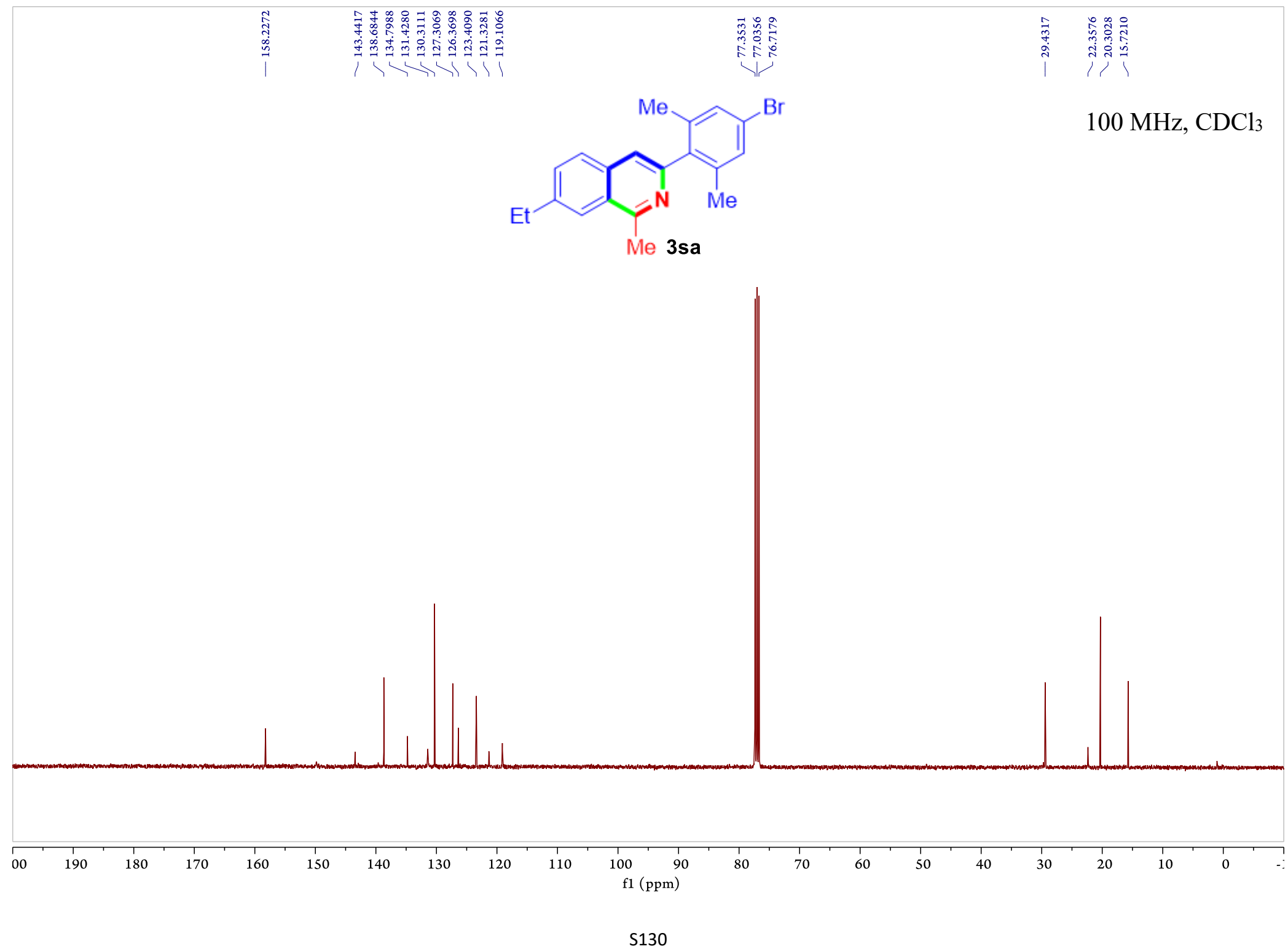




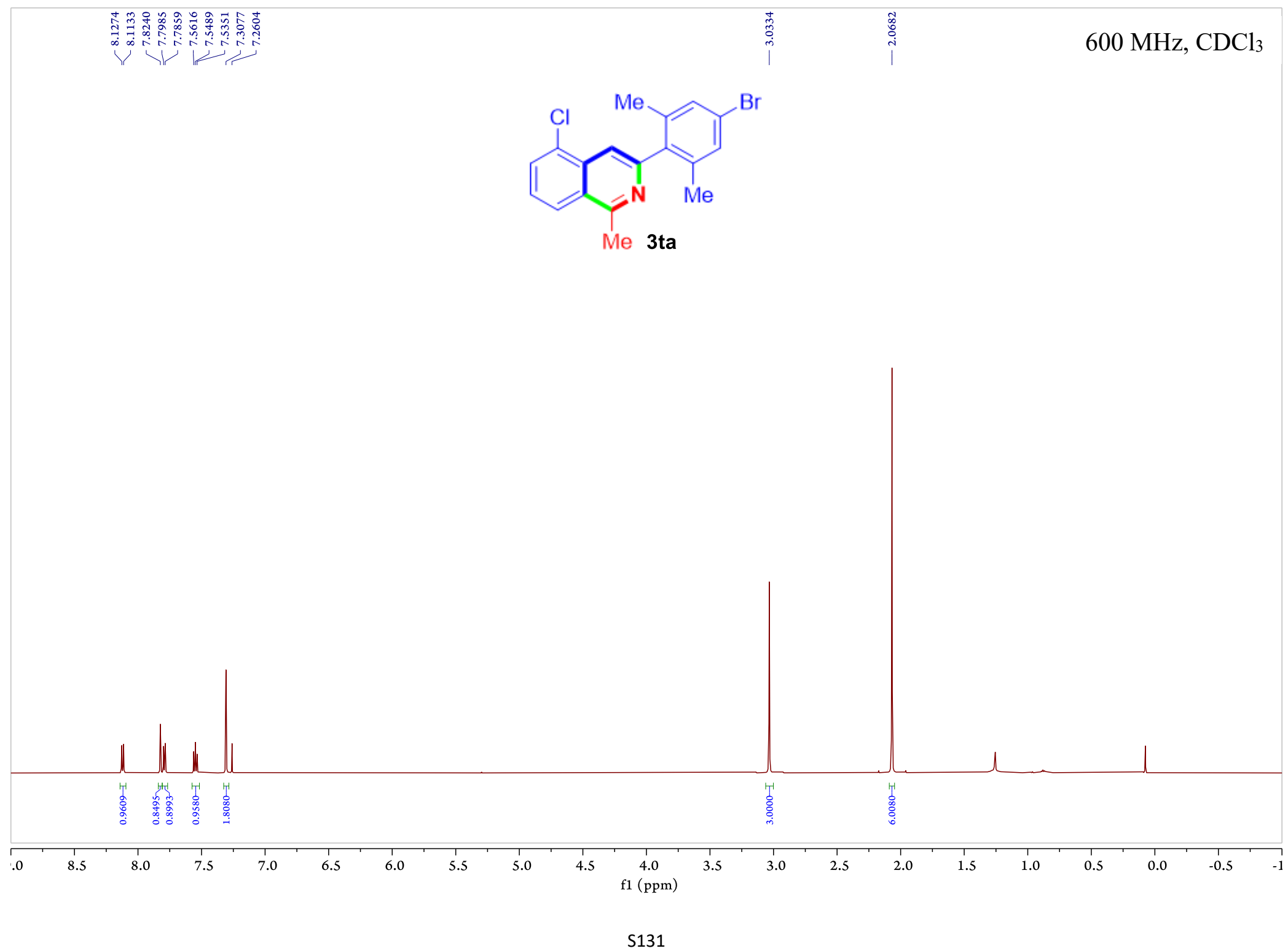




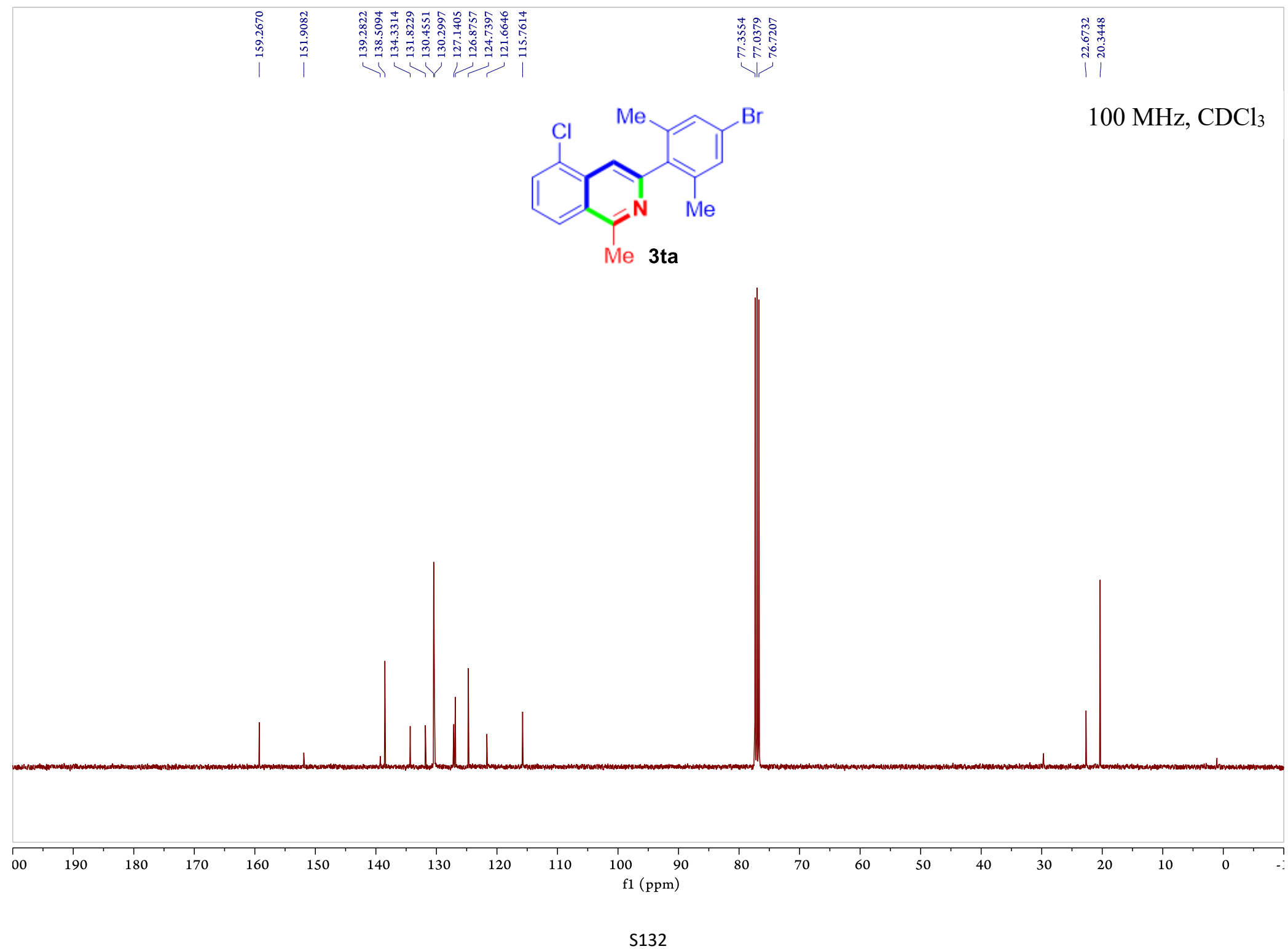




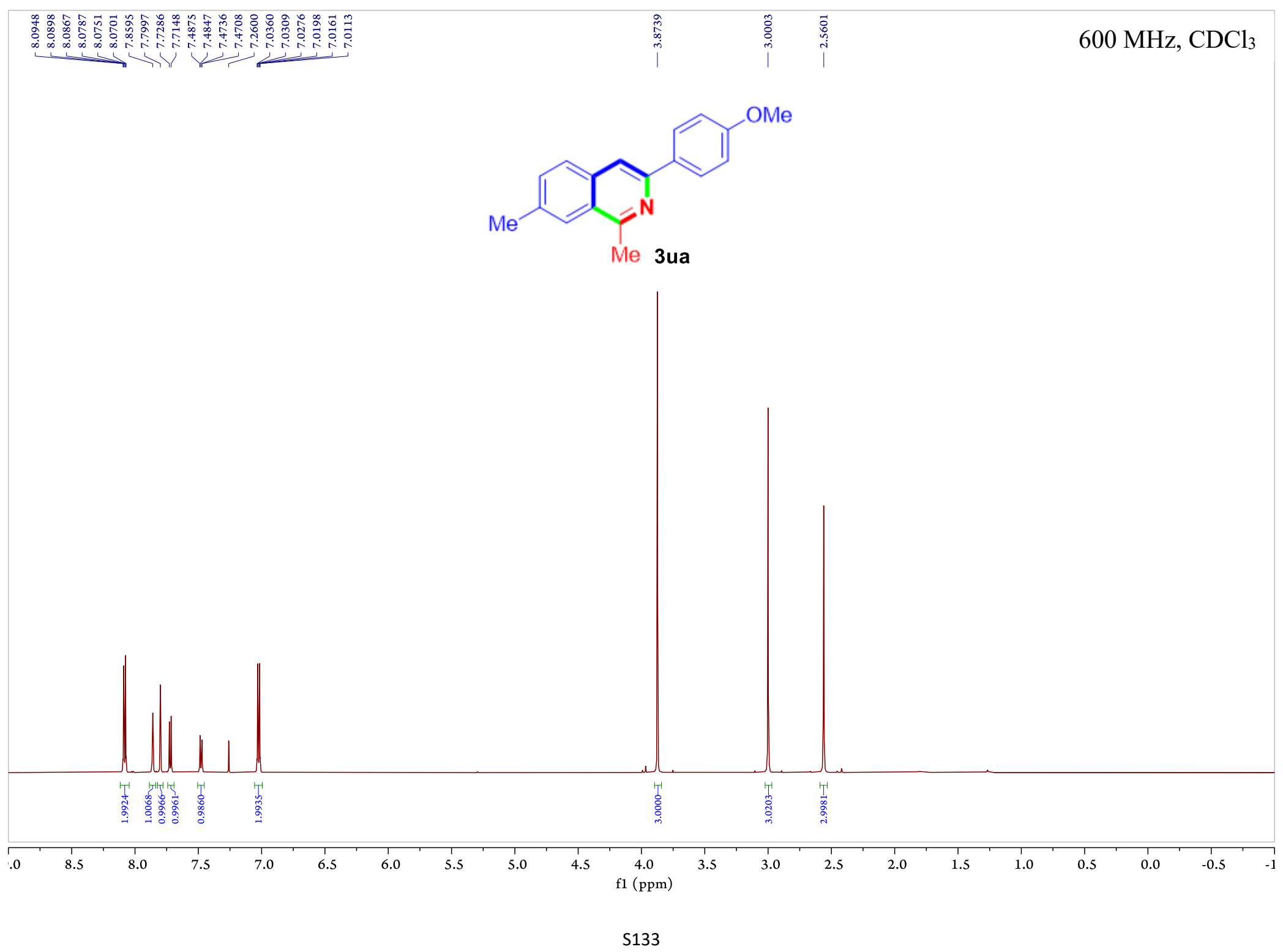




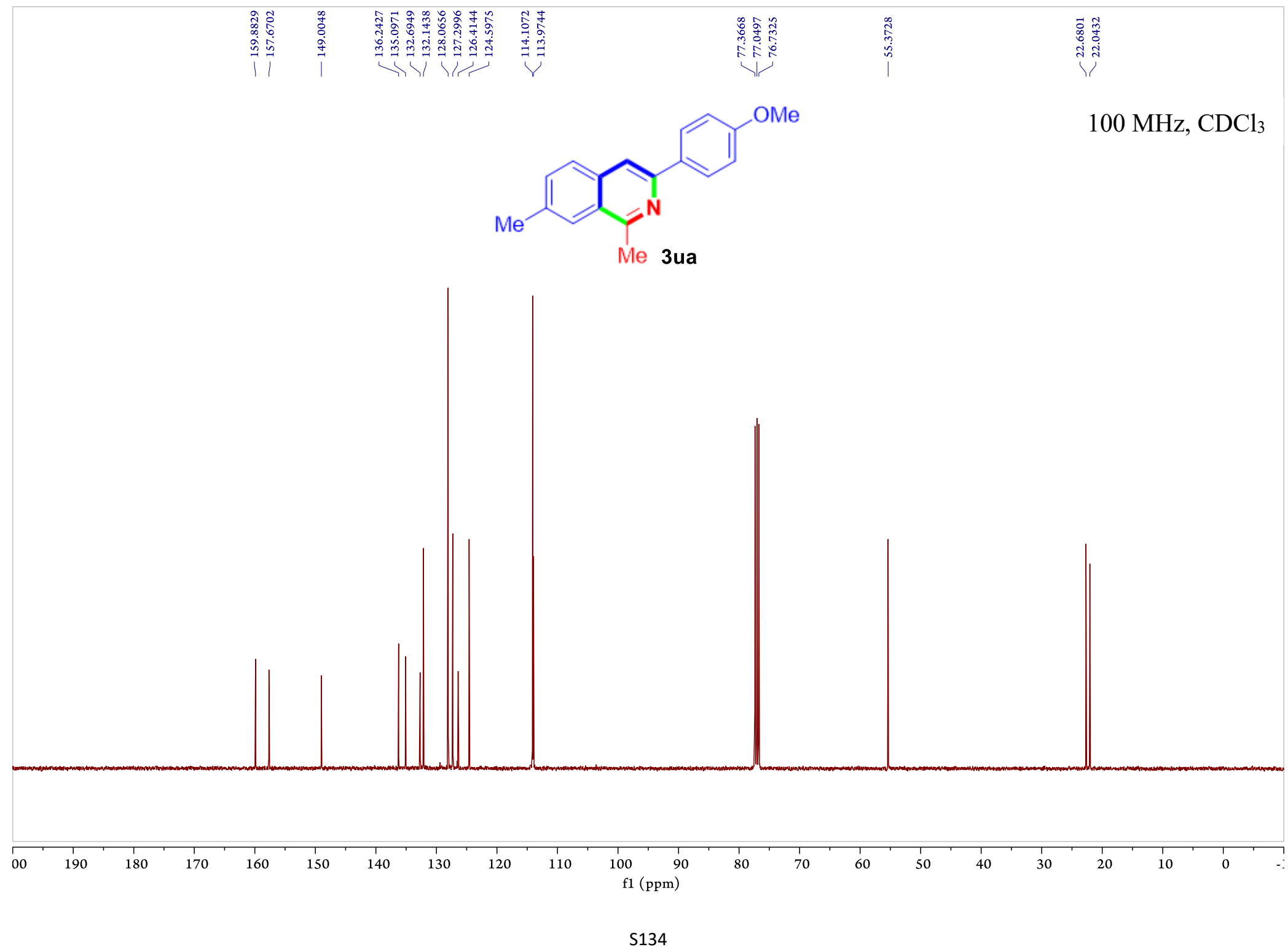




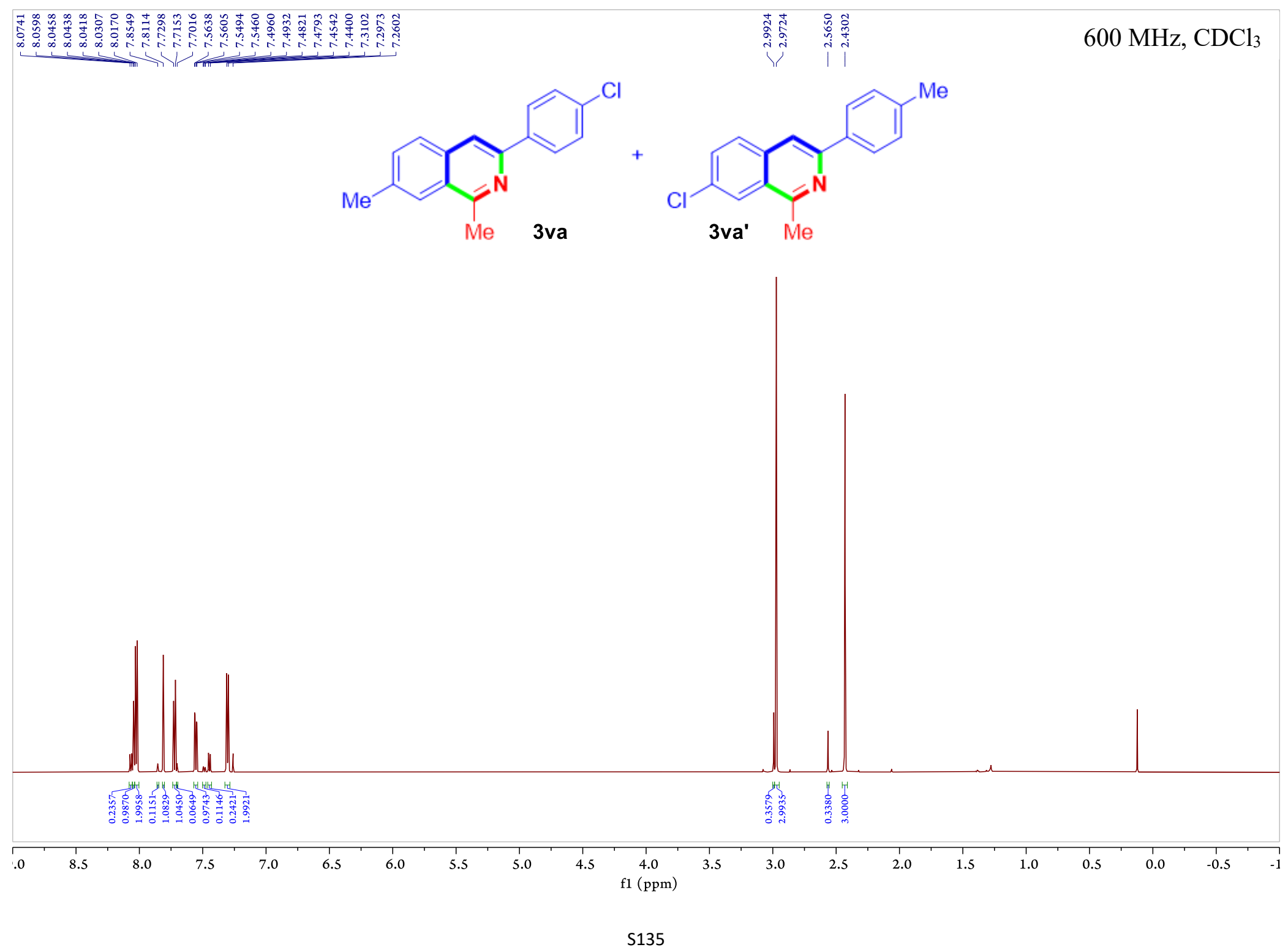




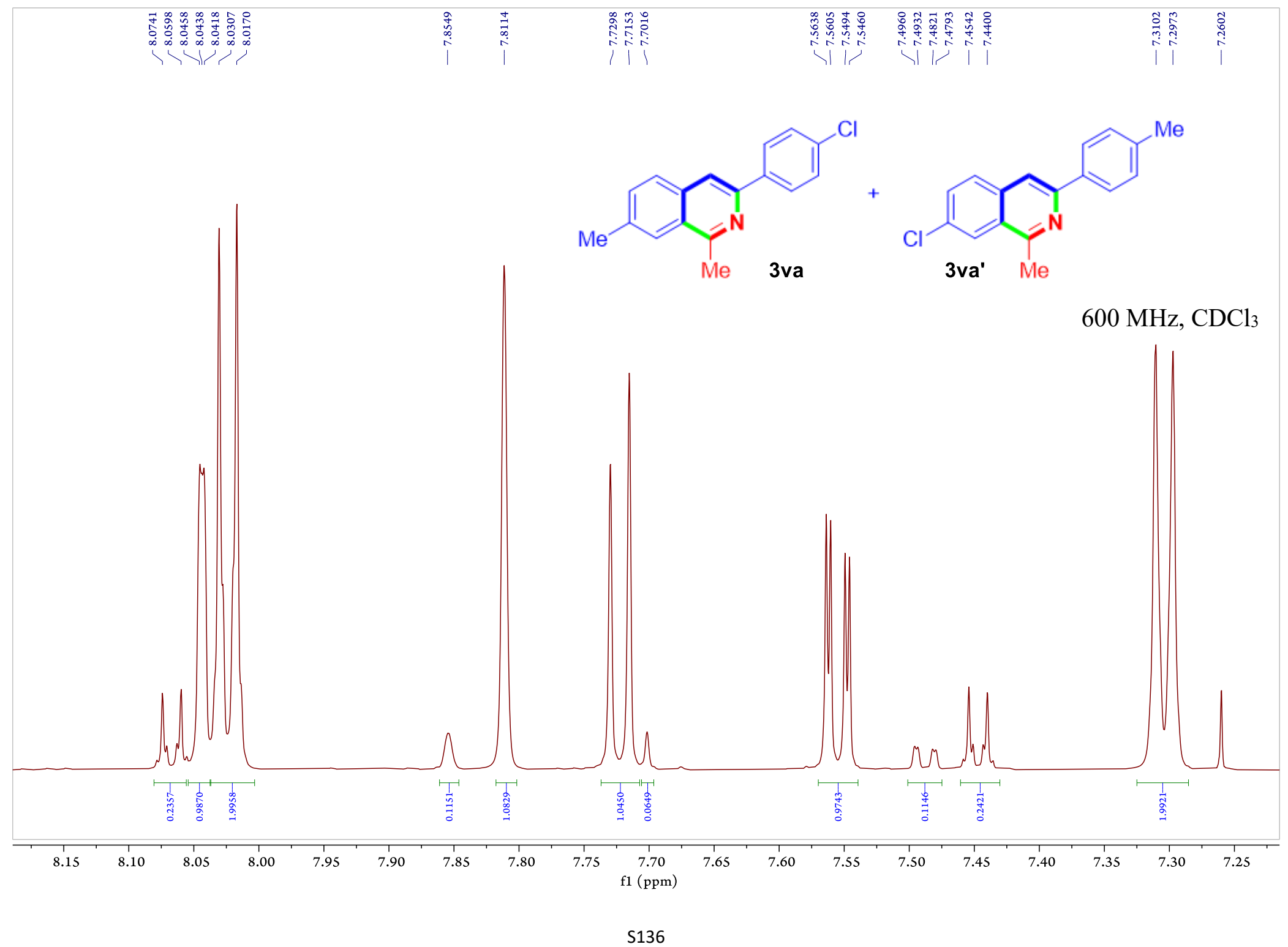




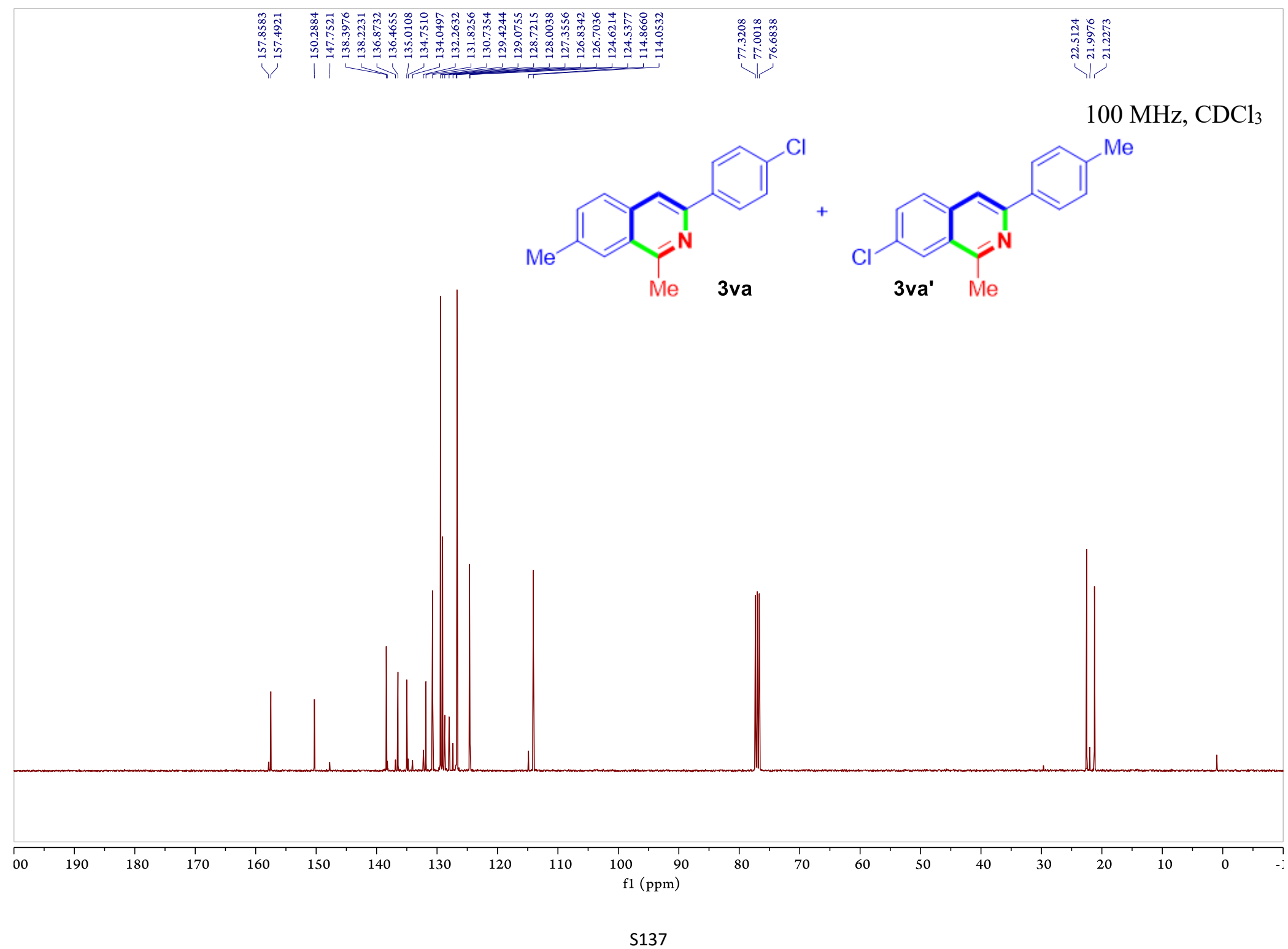




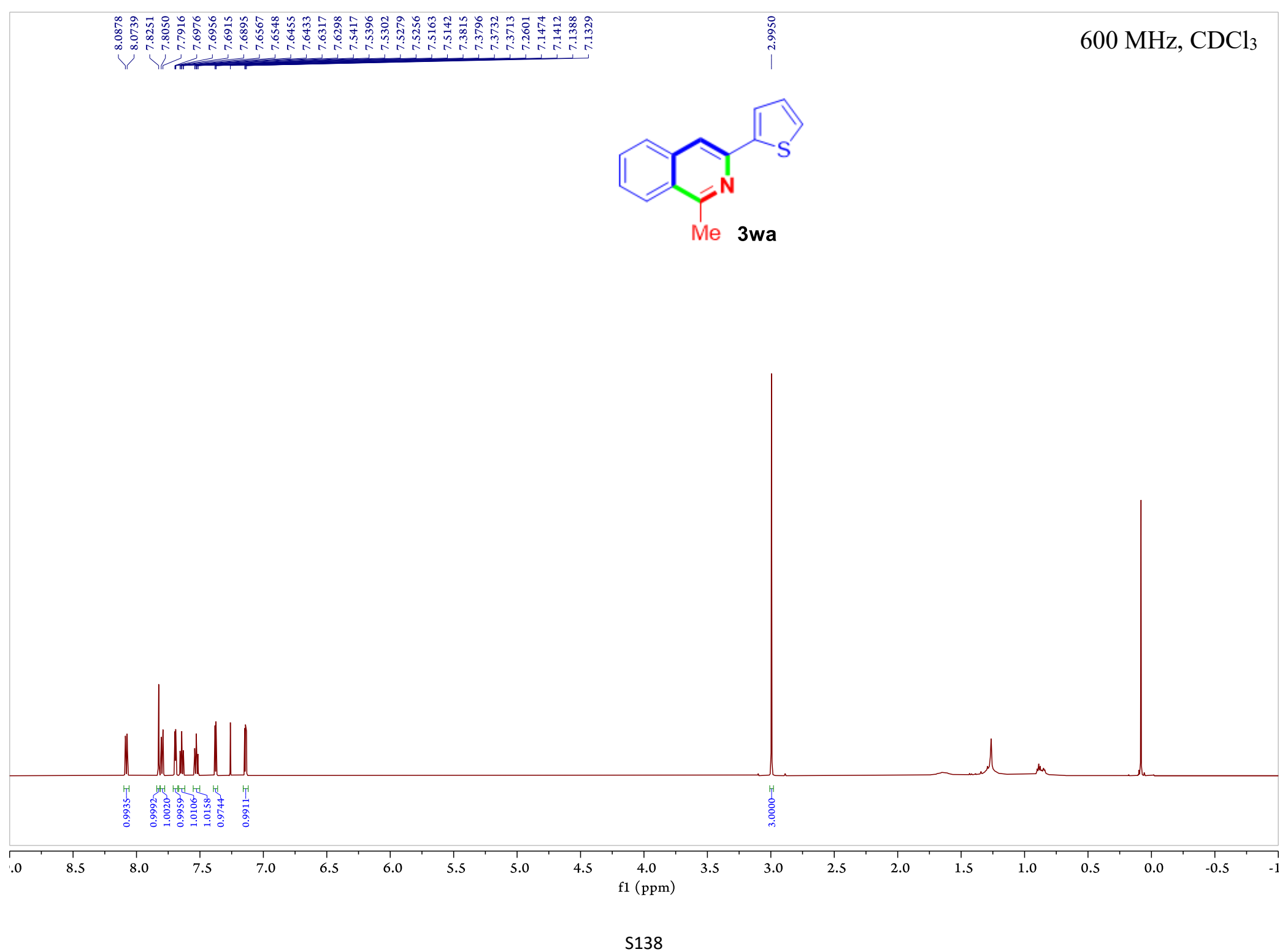




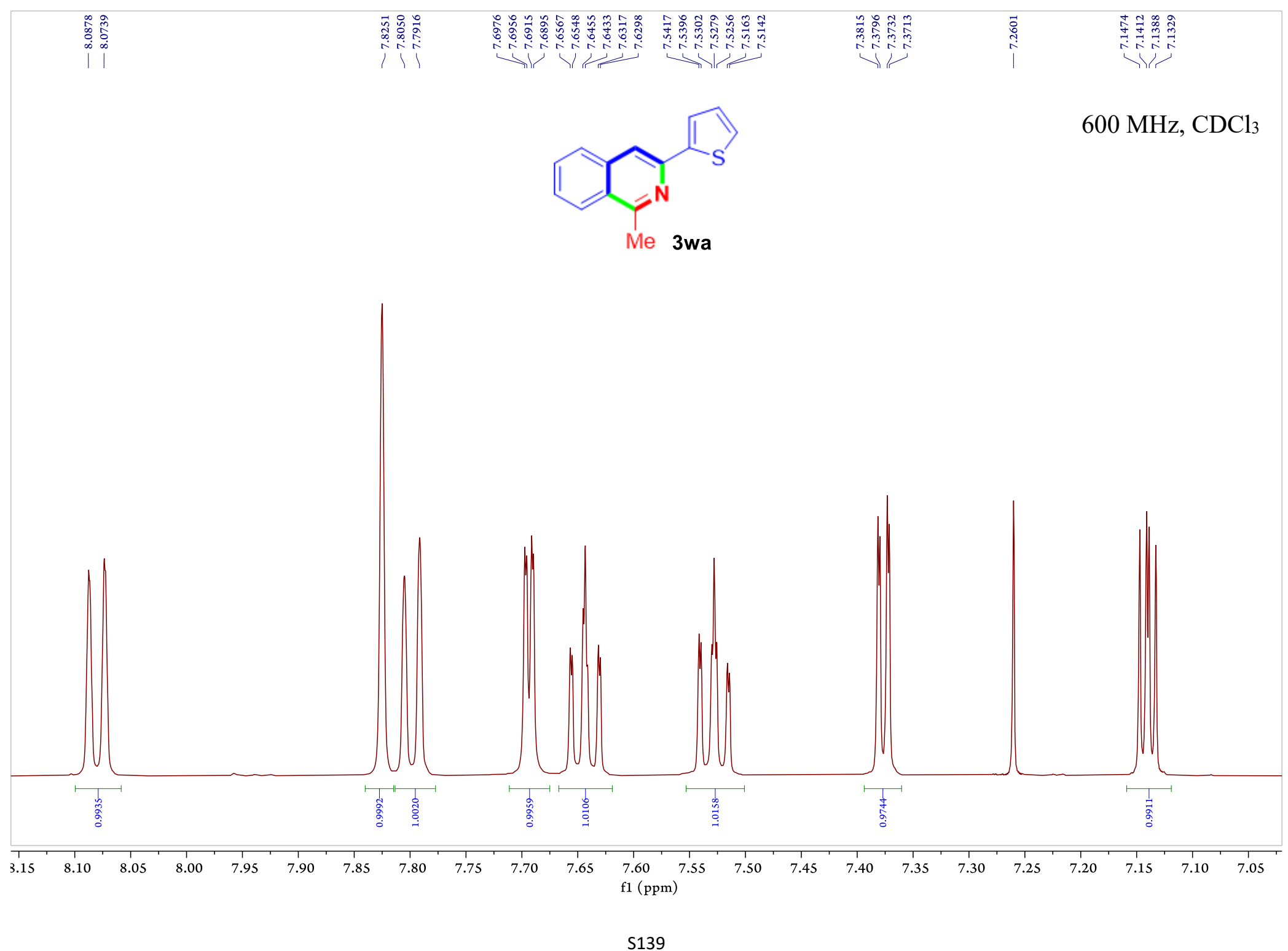




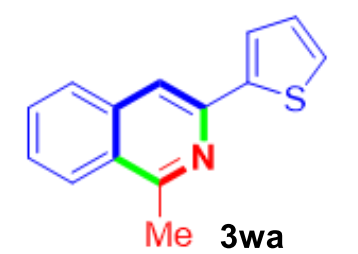

$100 \mathrm{MHz}, \mathrm{CDCl}_{3}$

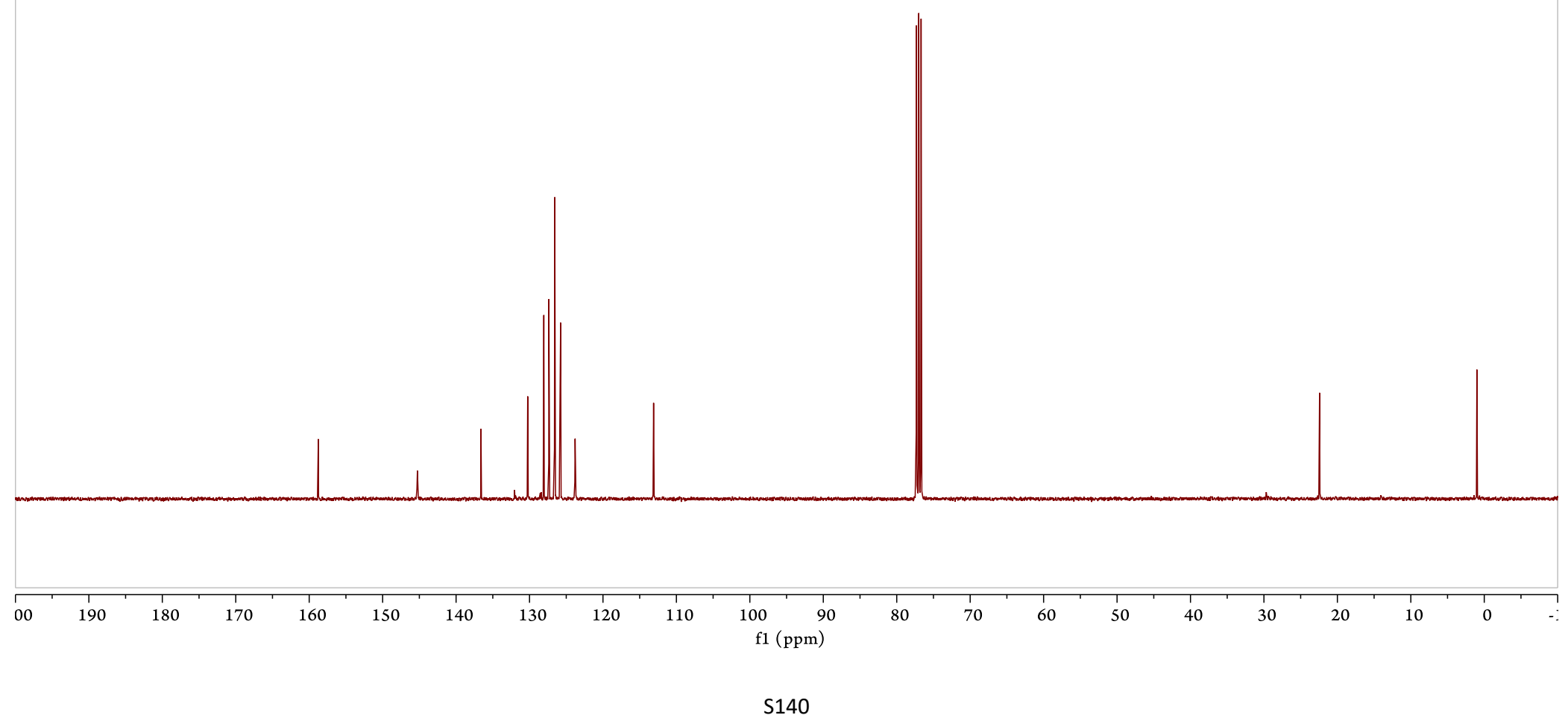




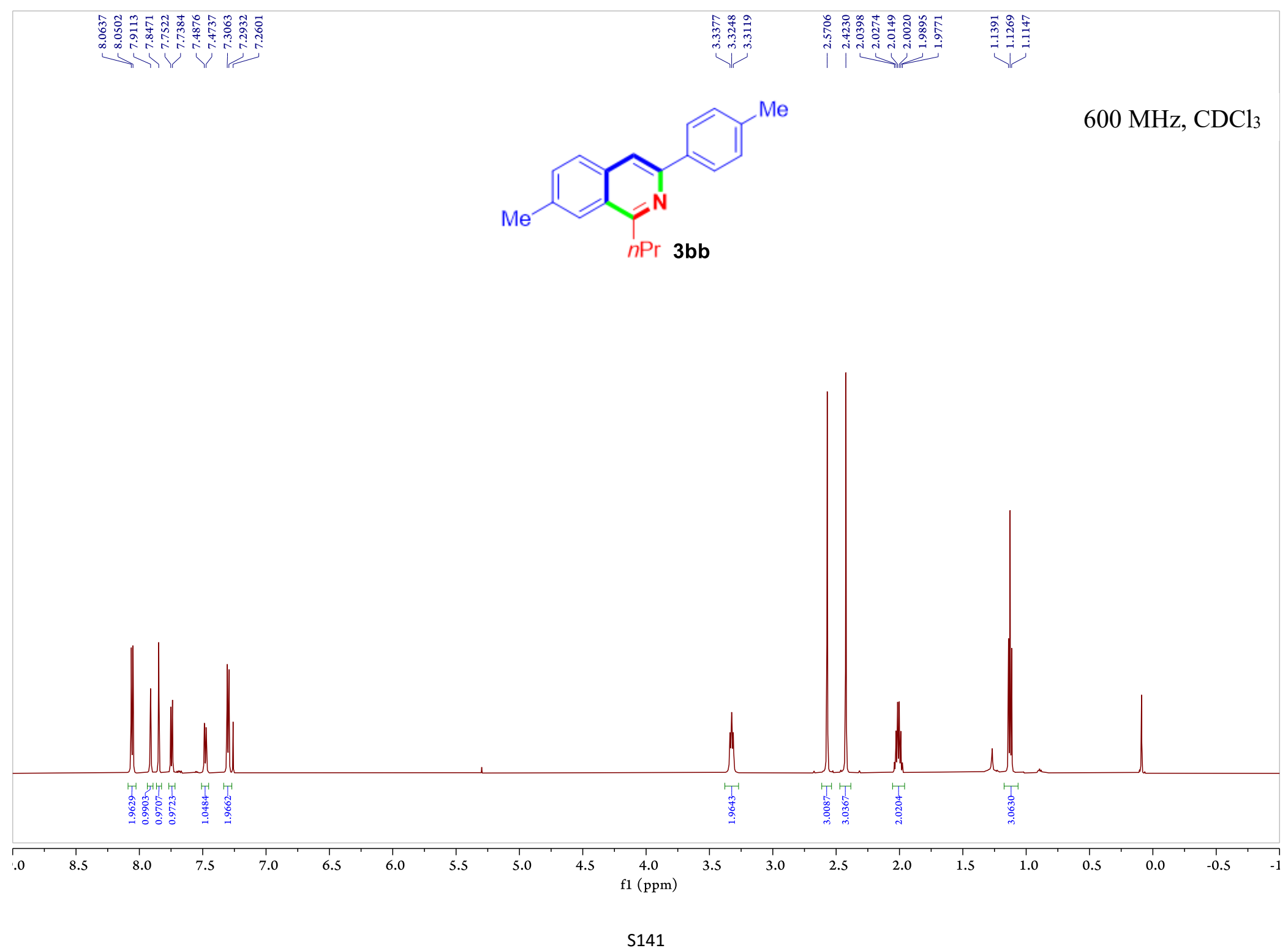




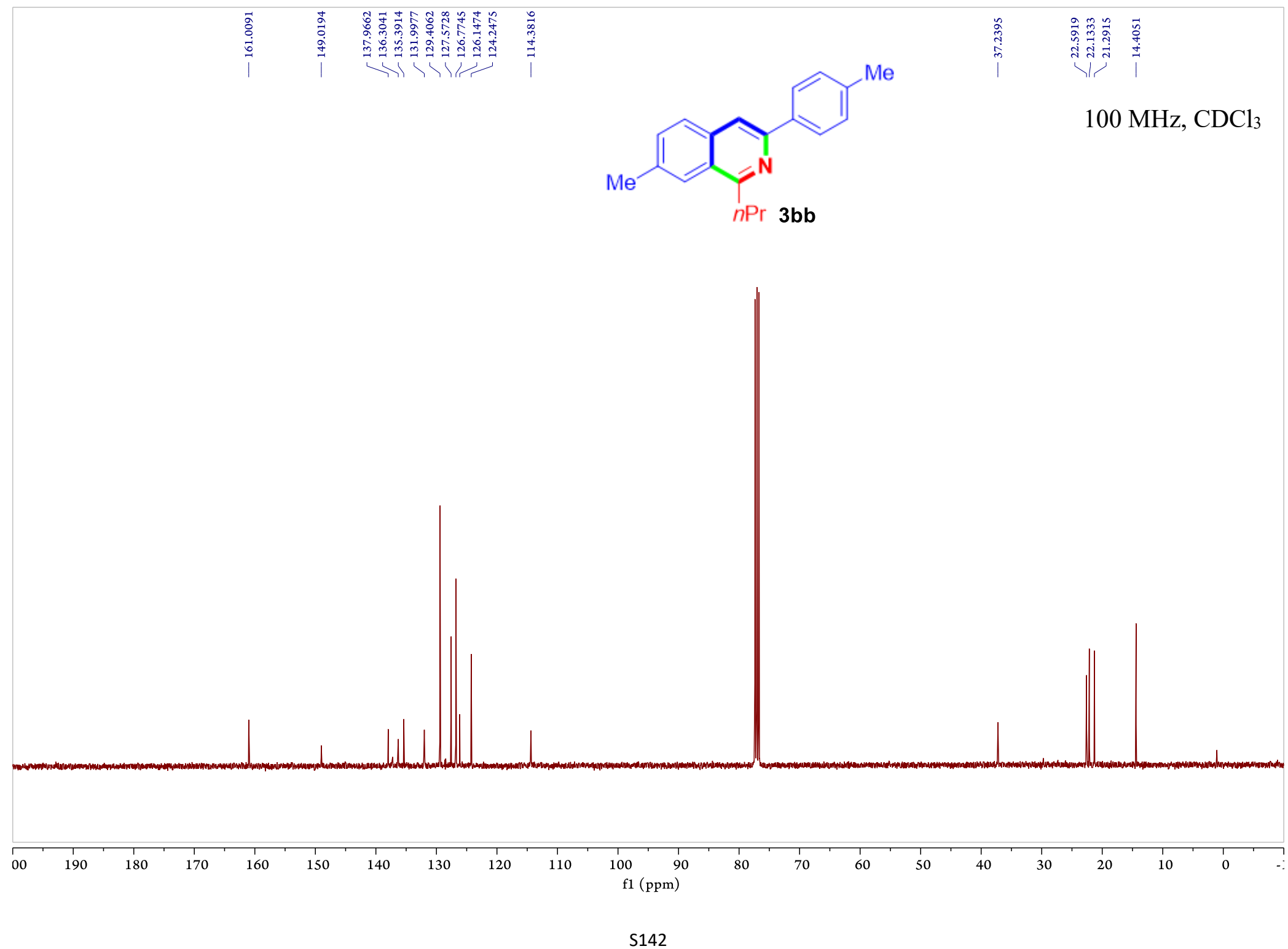




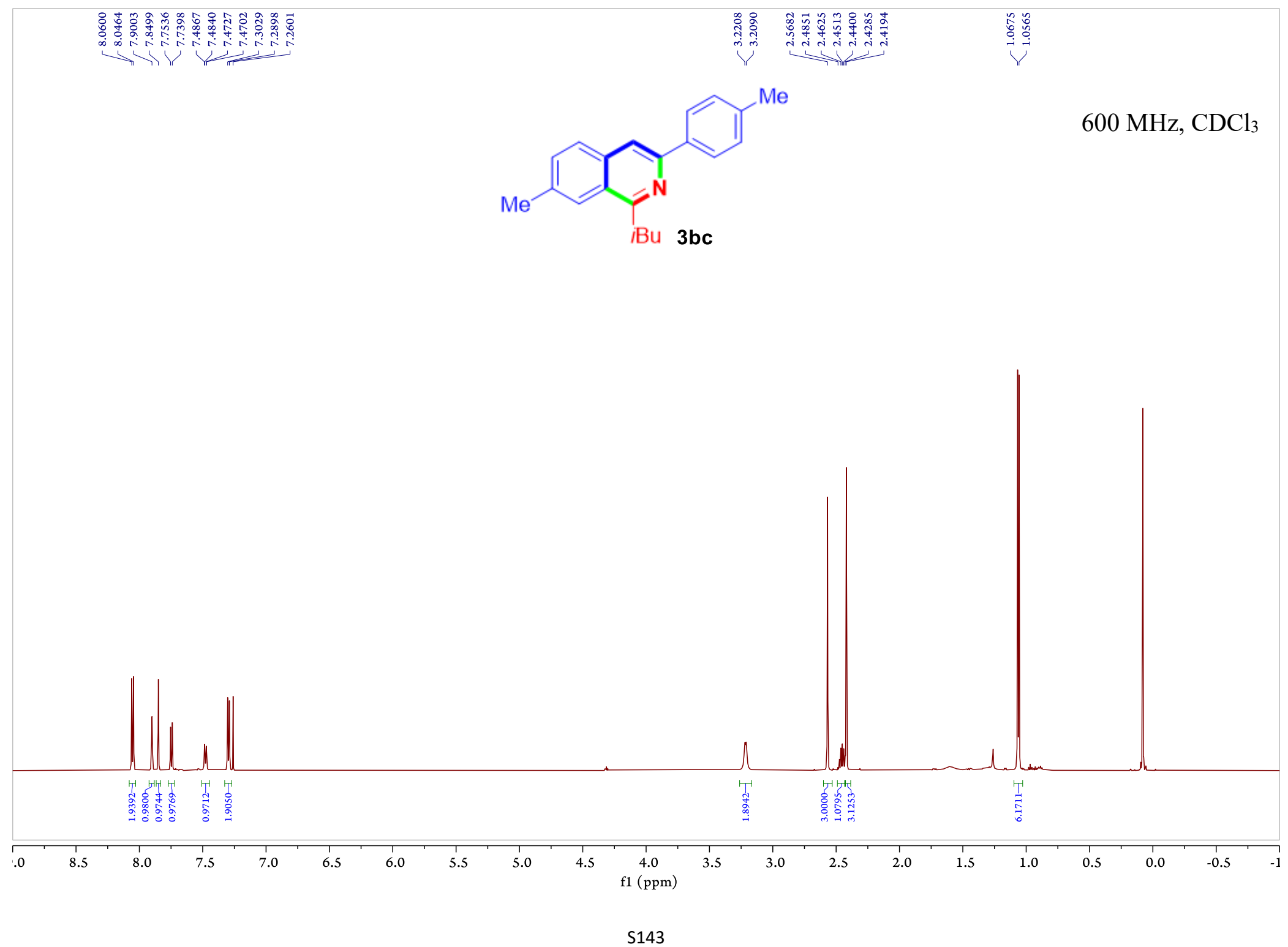




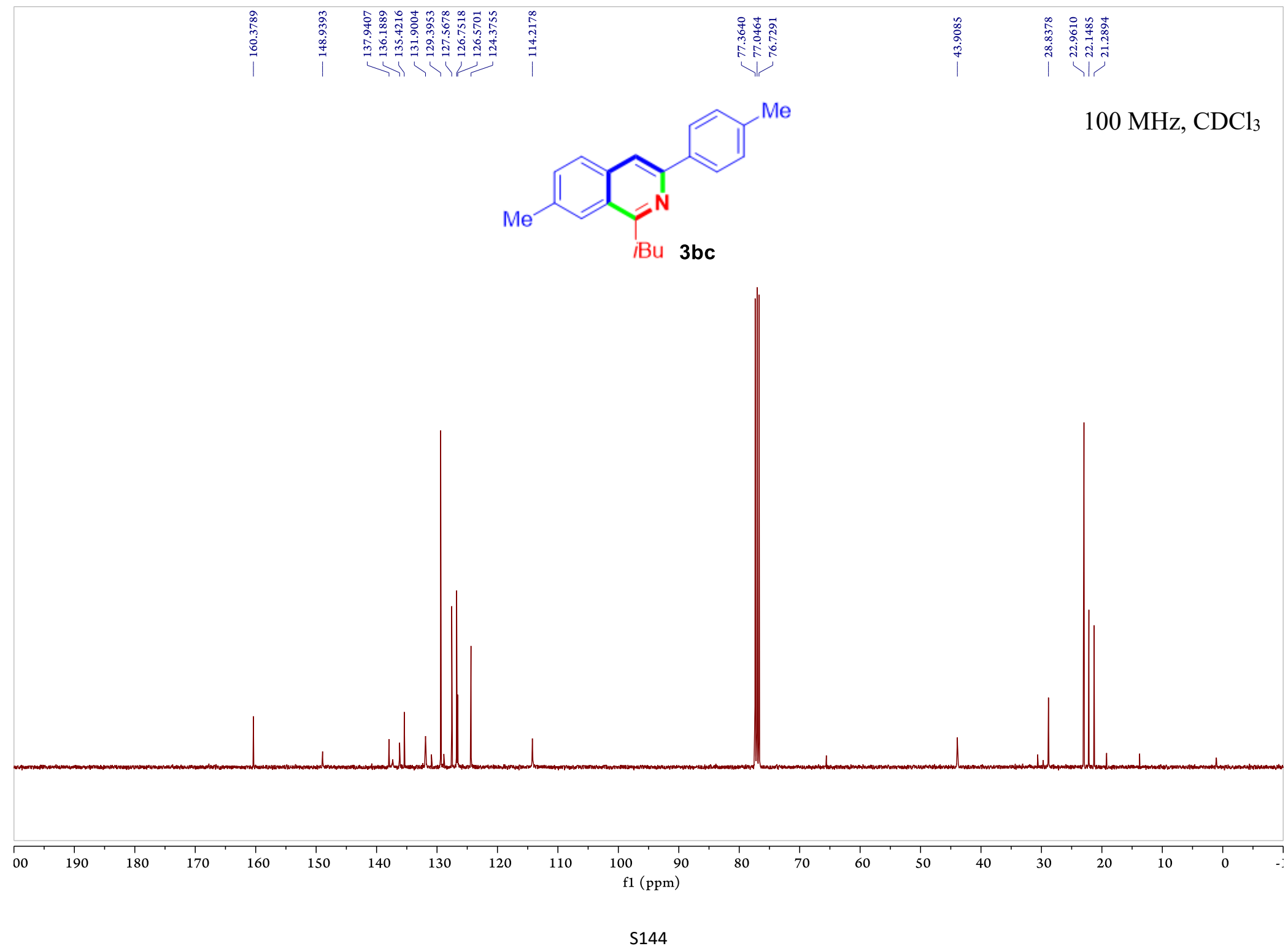




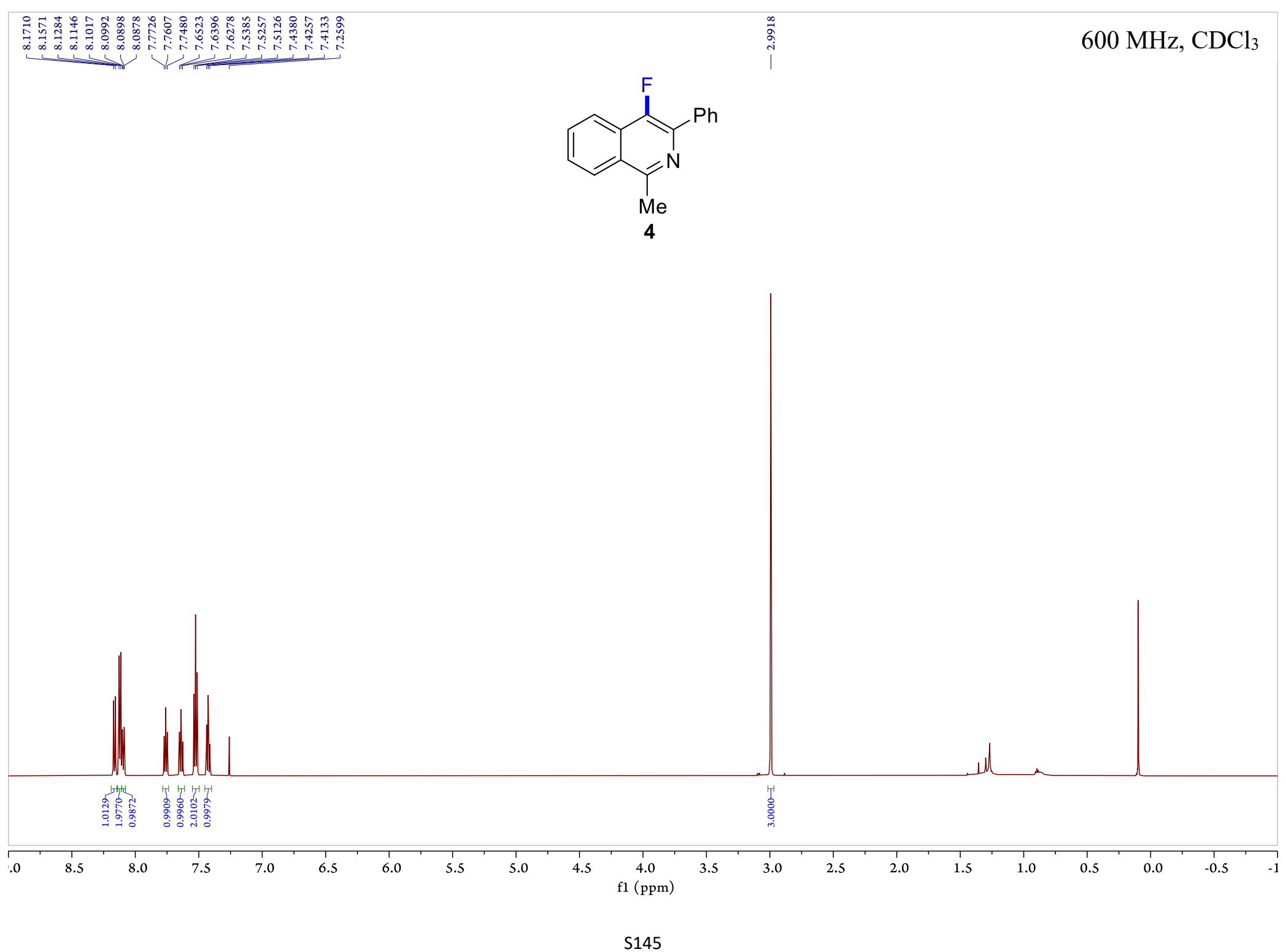




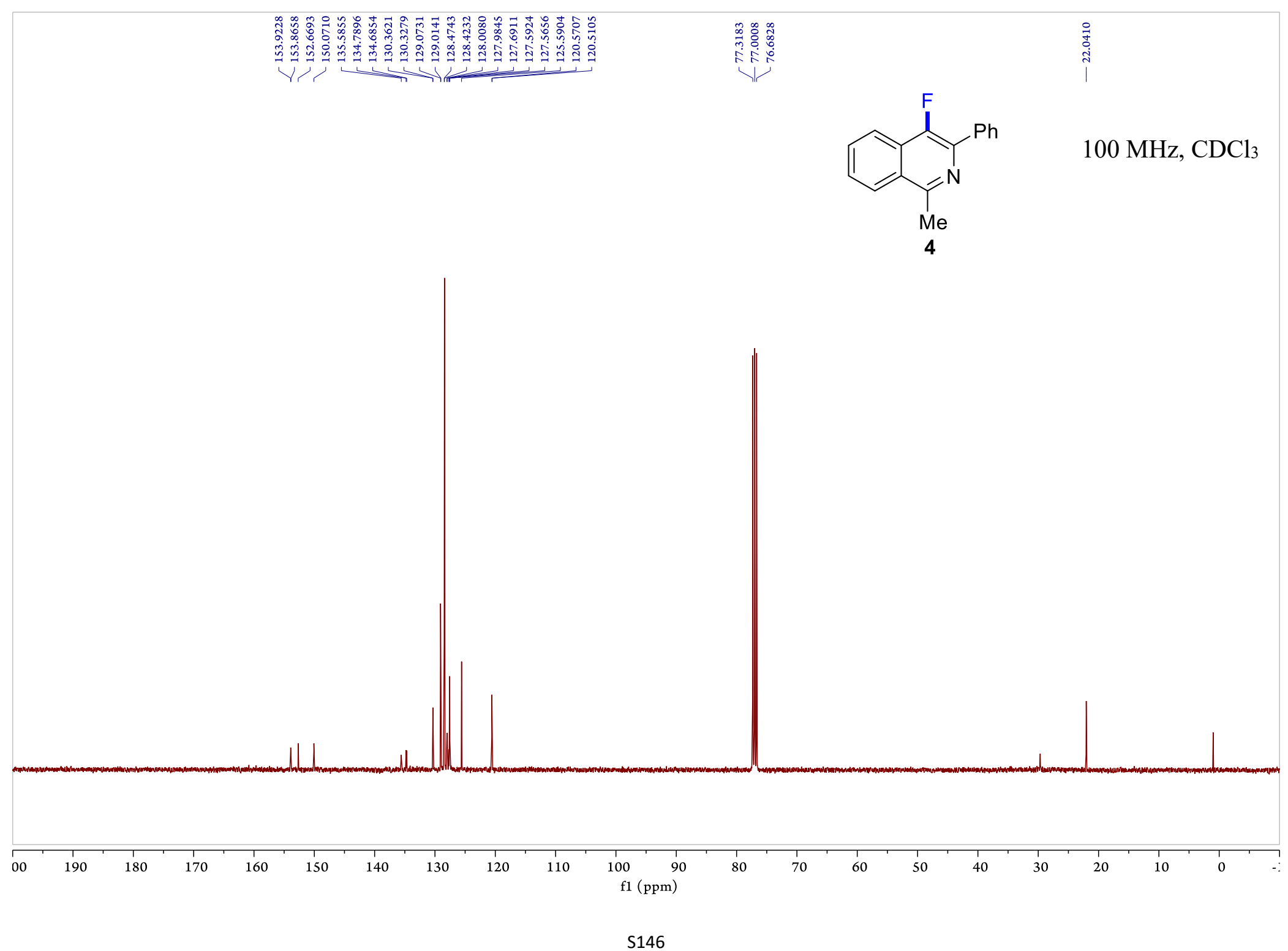




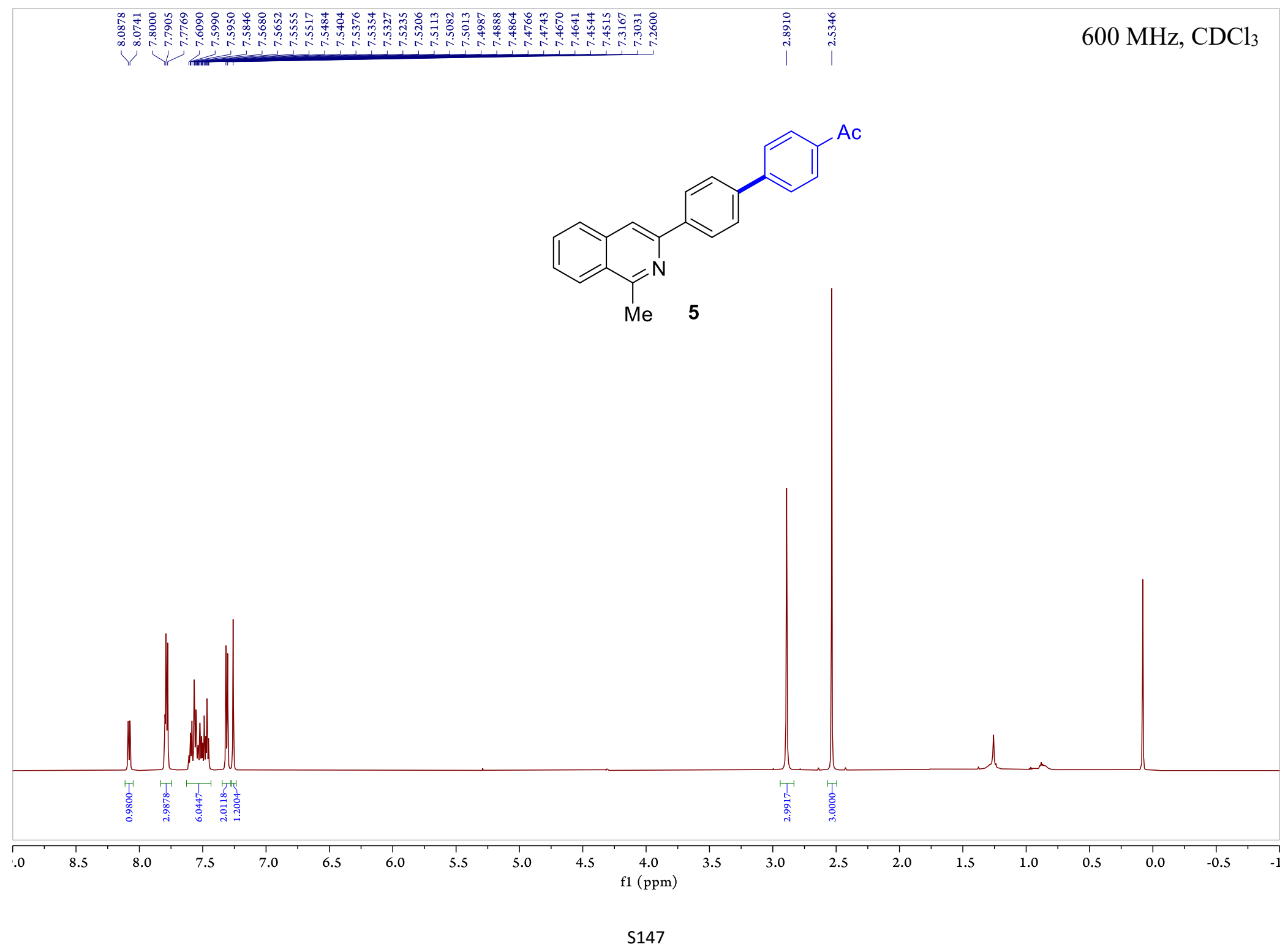




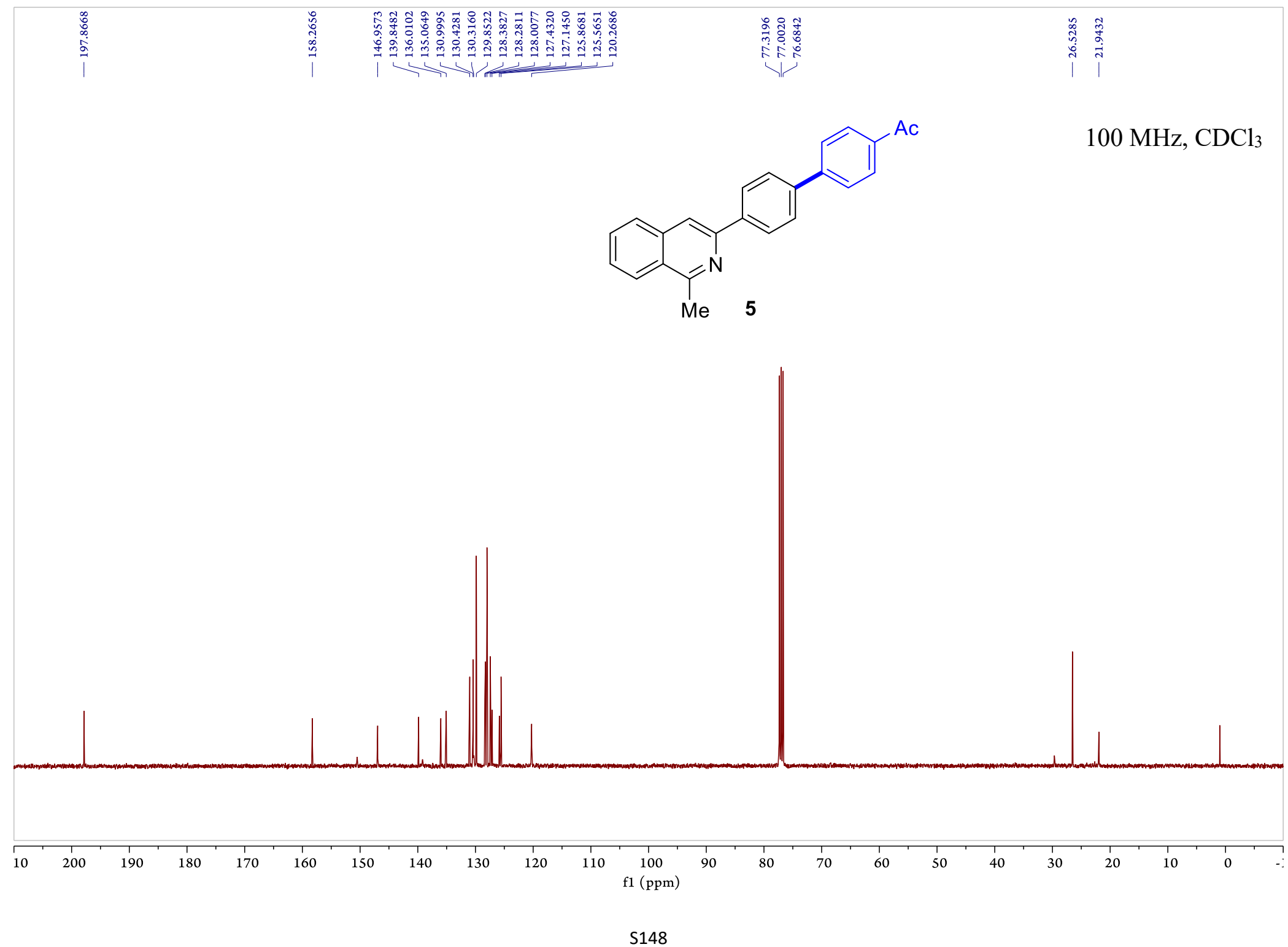




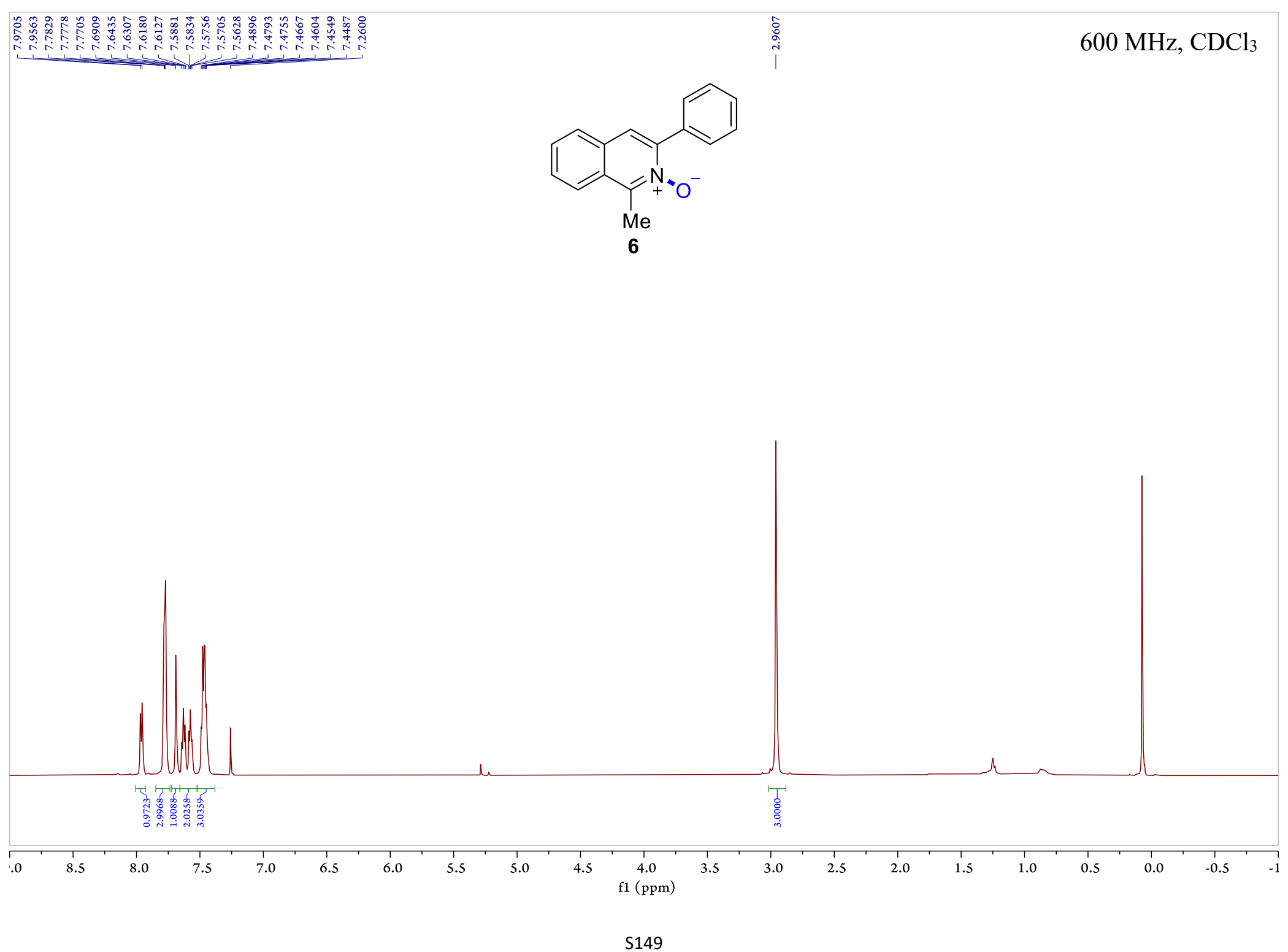




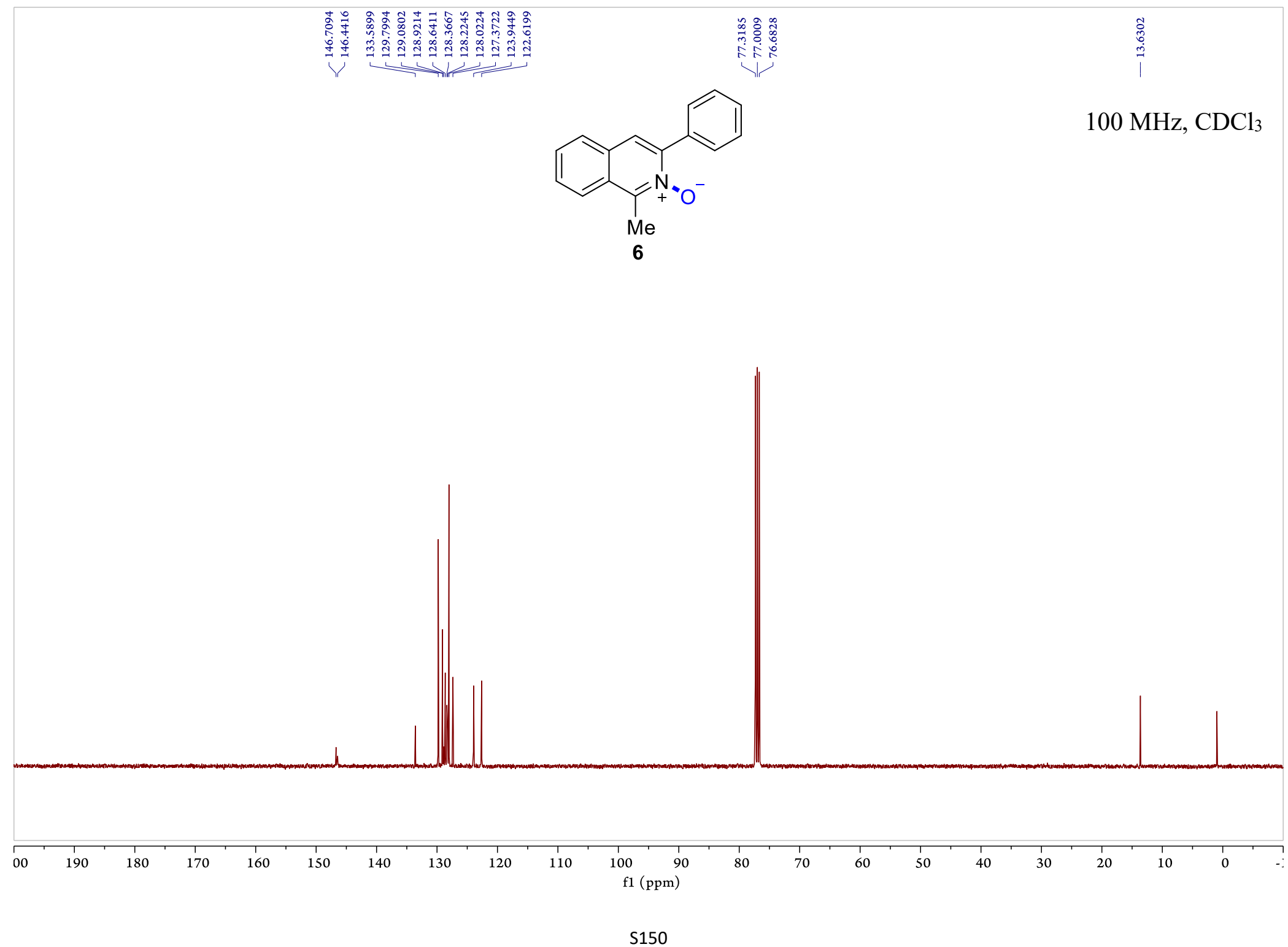




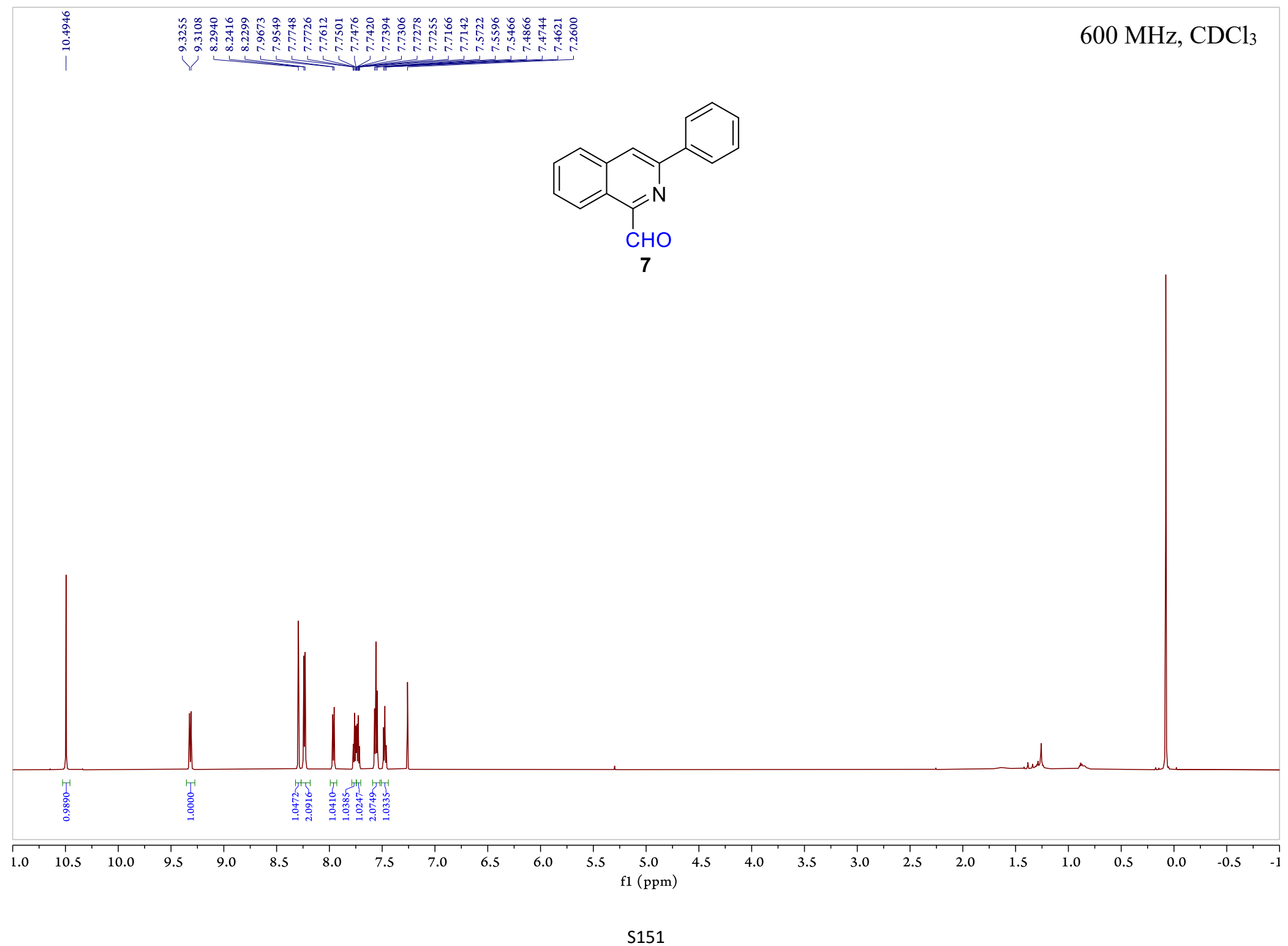




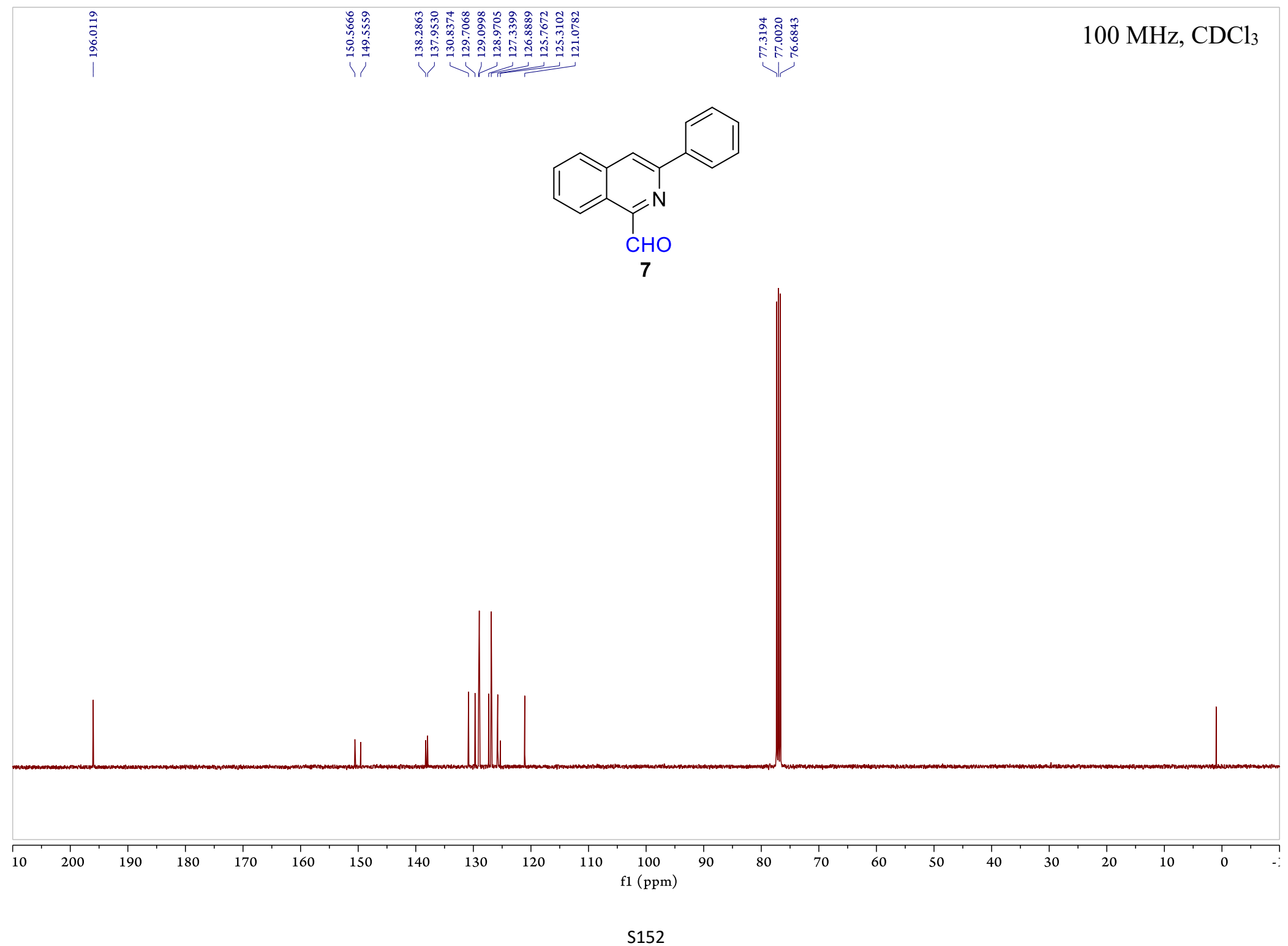




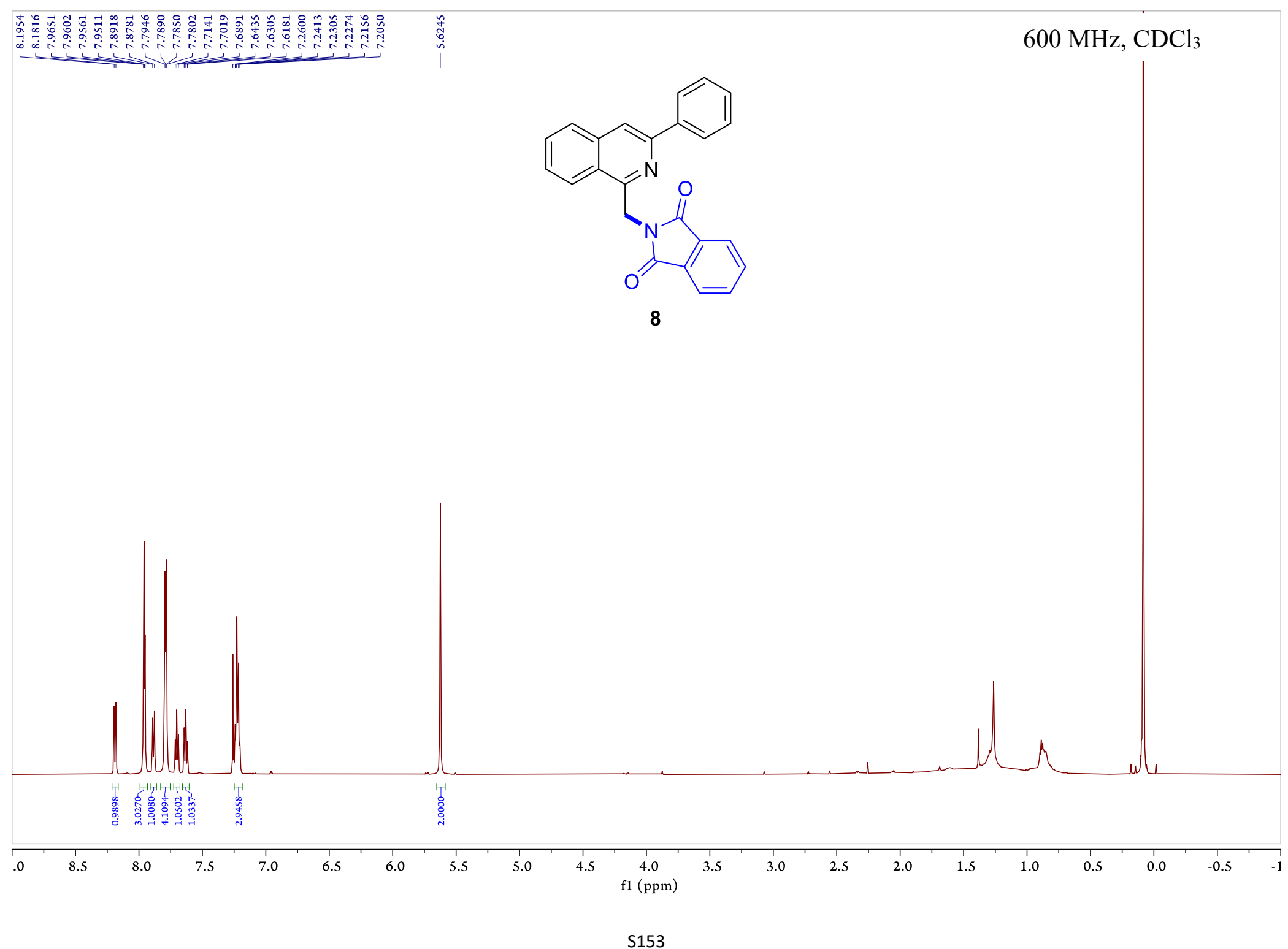




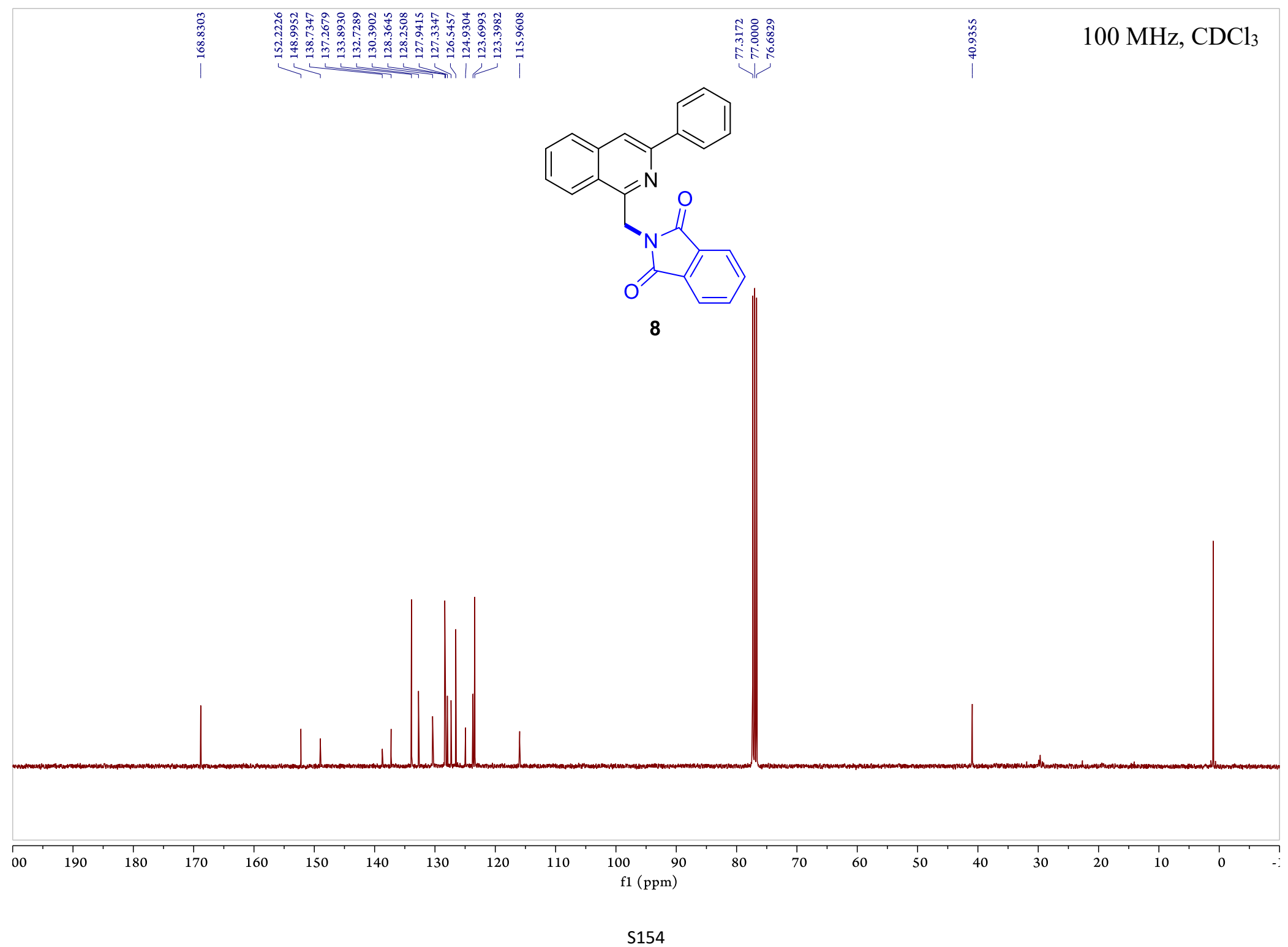




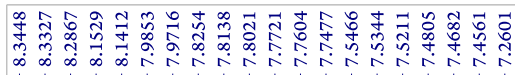

$600 \mathrm{MHz} \mathrm{CDCl}_{3}$
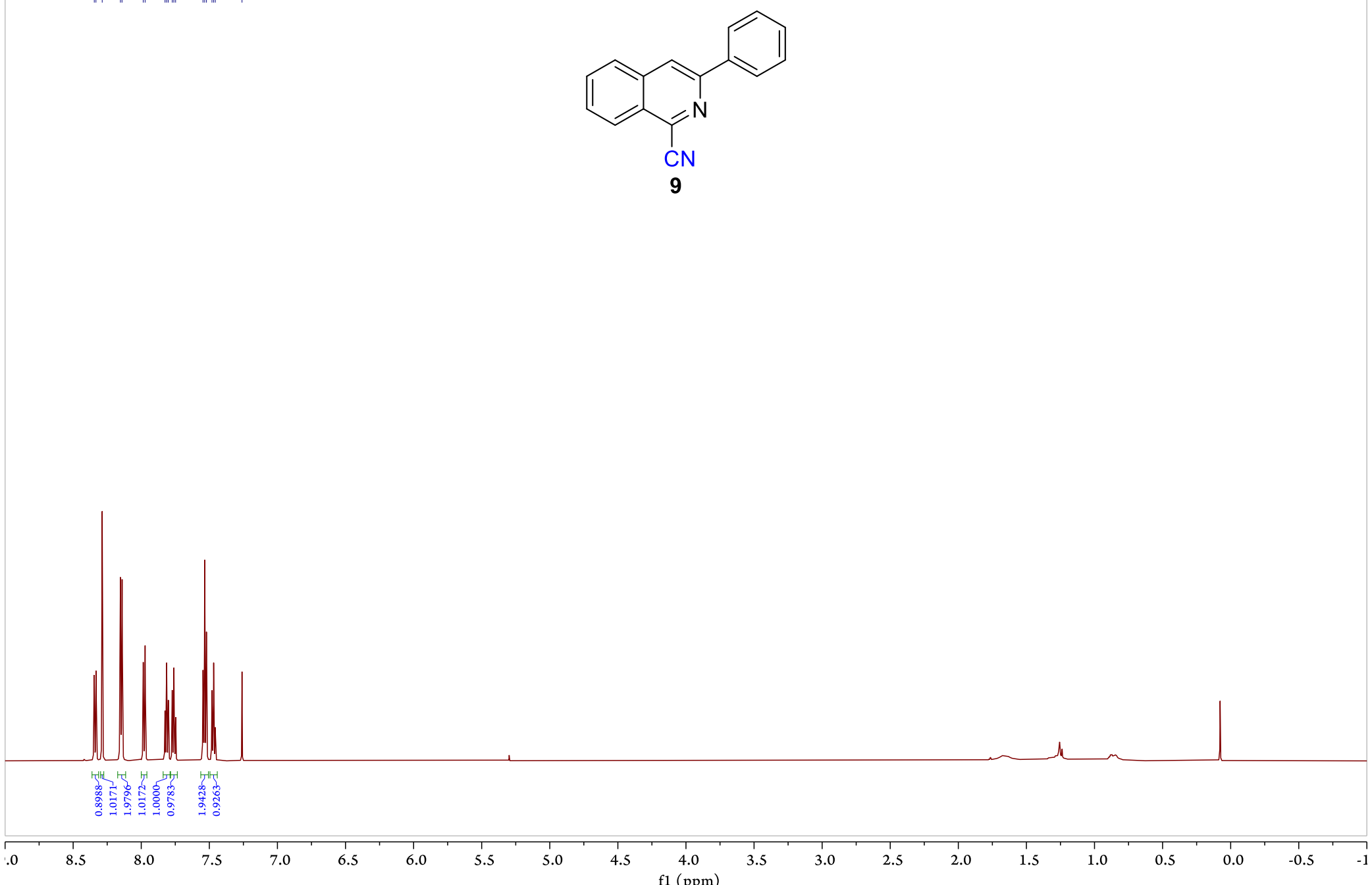


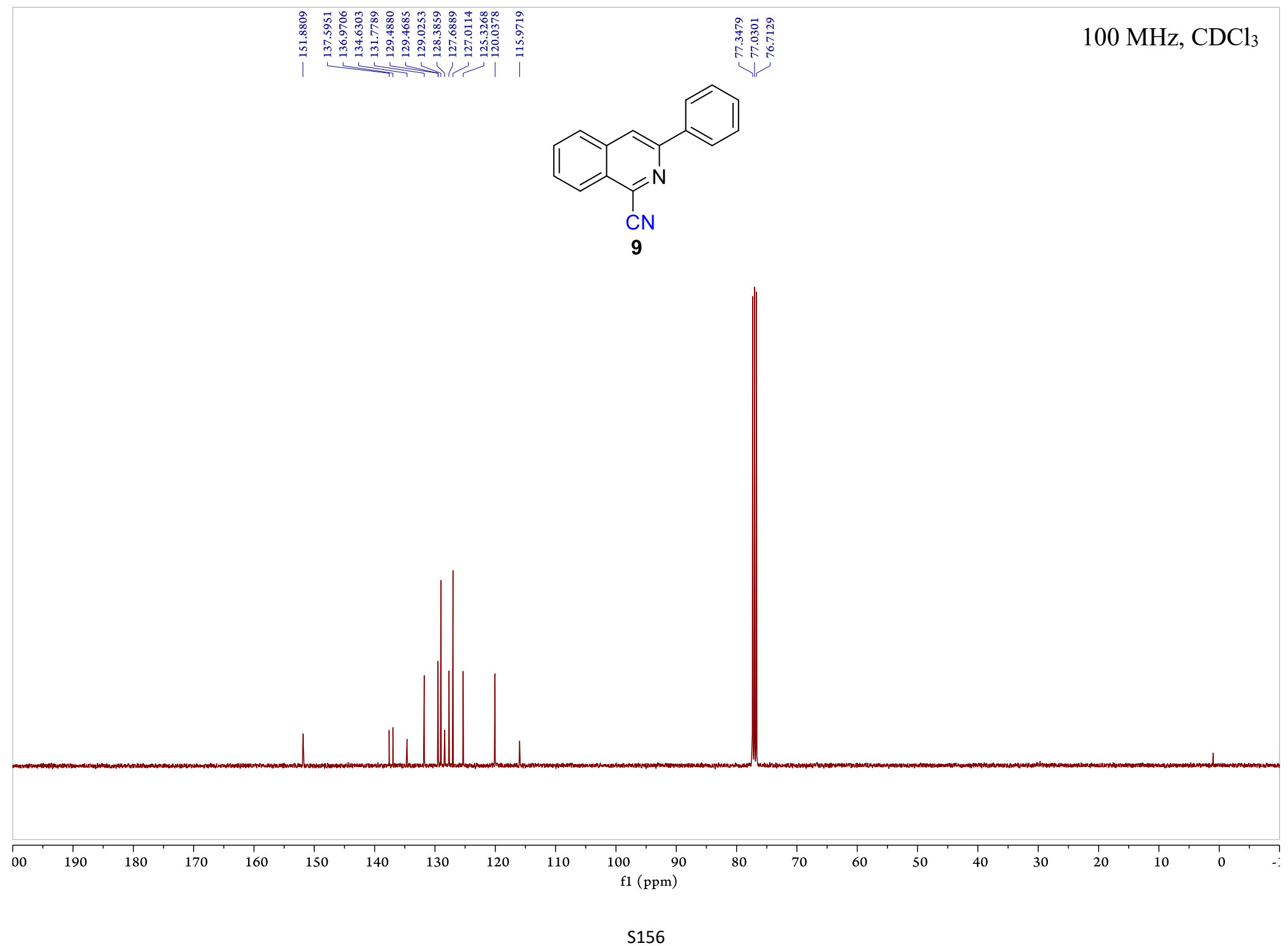




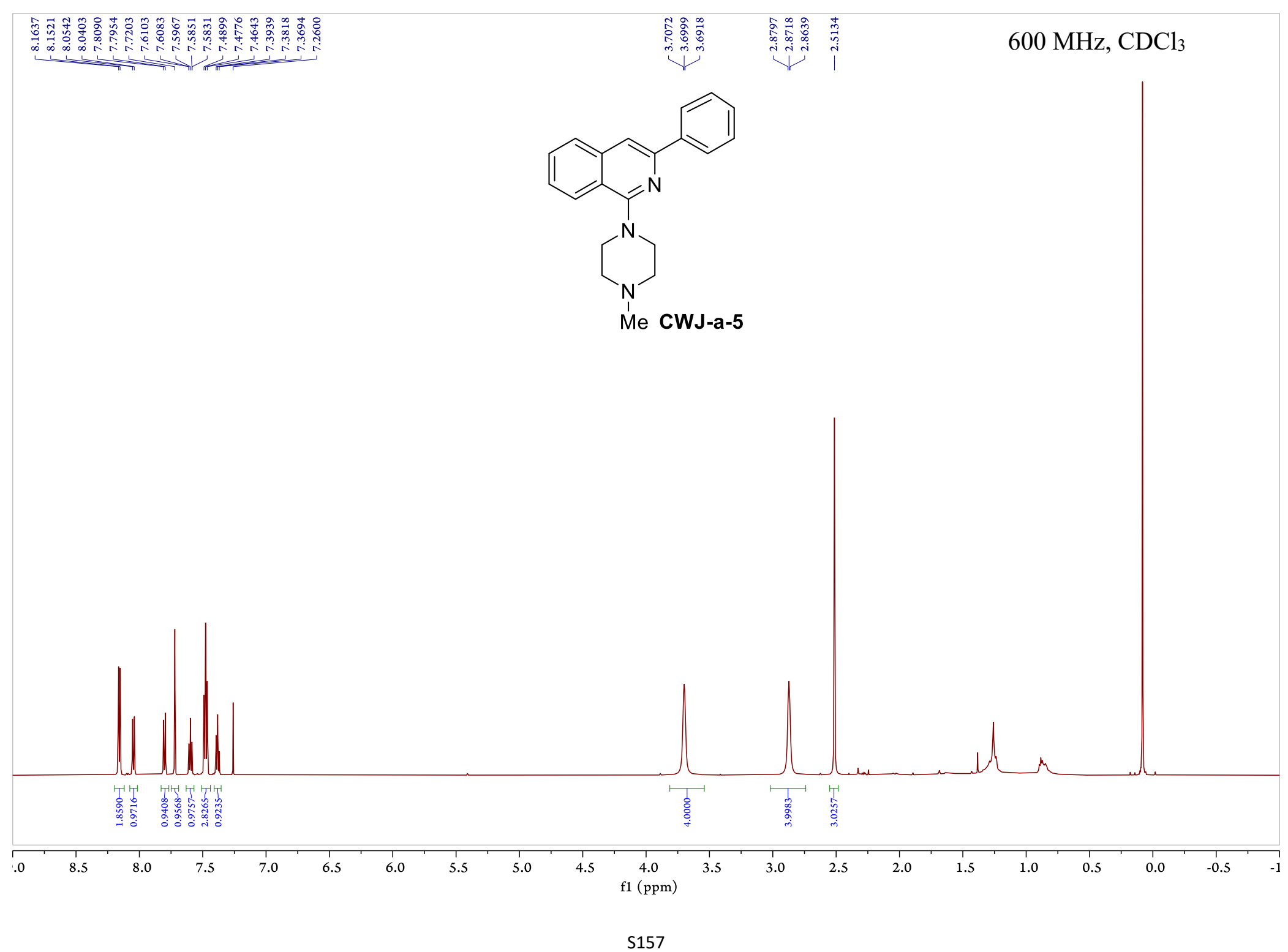




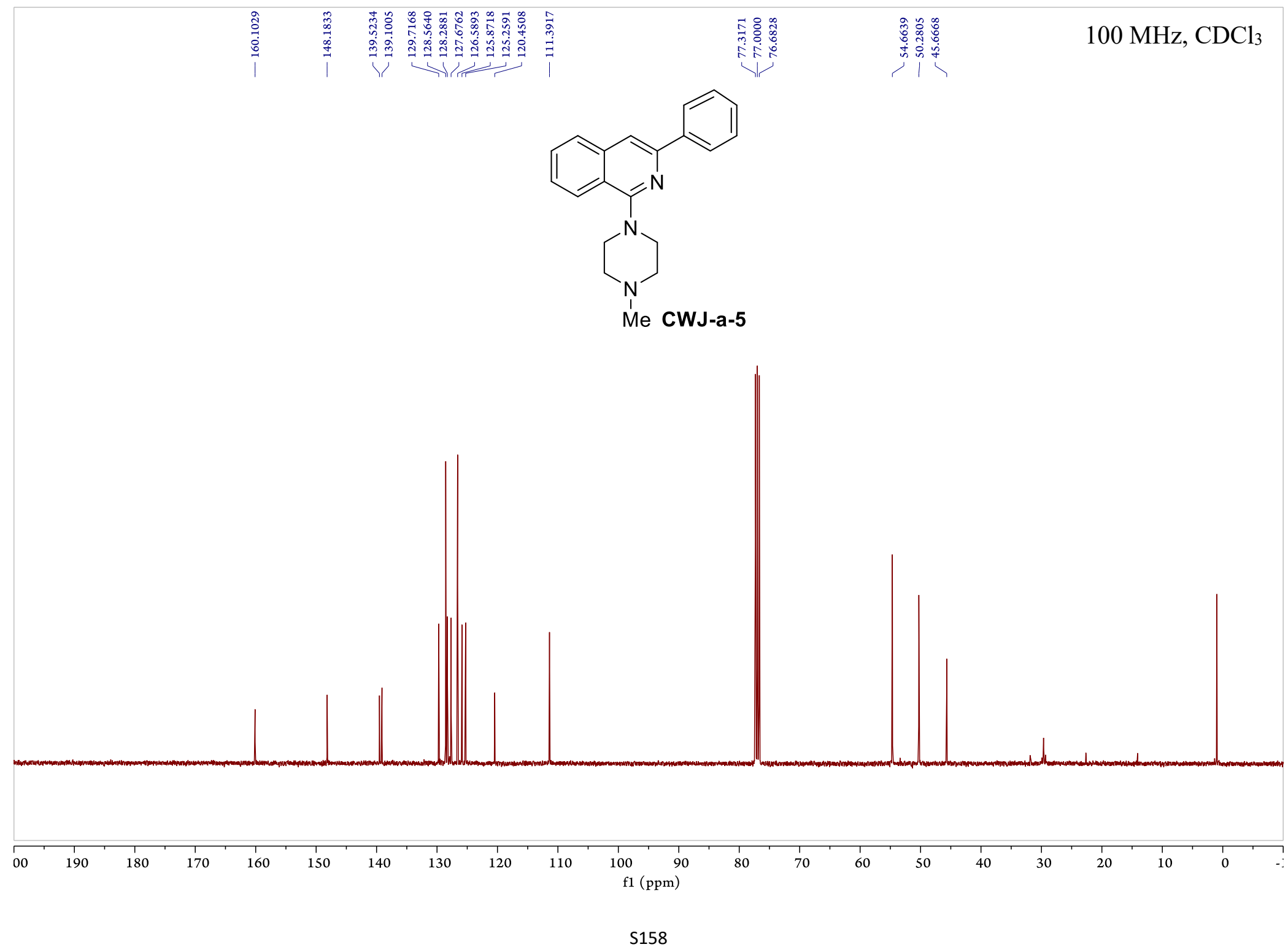




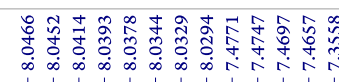

罚

$600 \mathrm{MHz}, \mathrm{CDCl}_{3}$
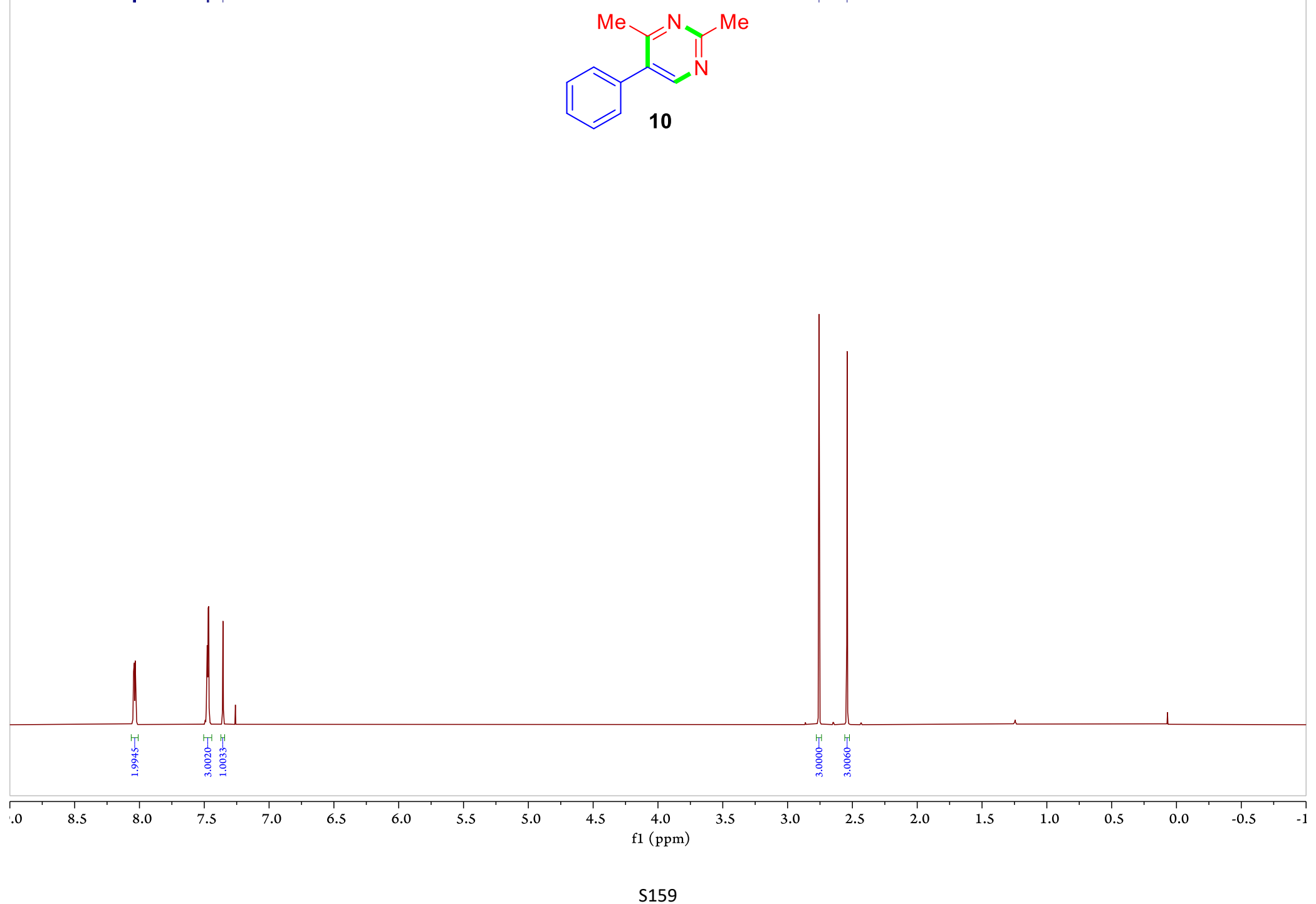


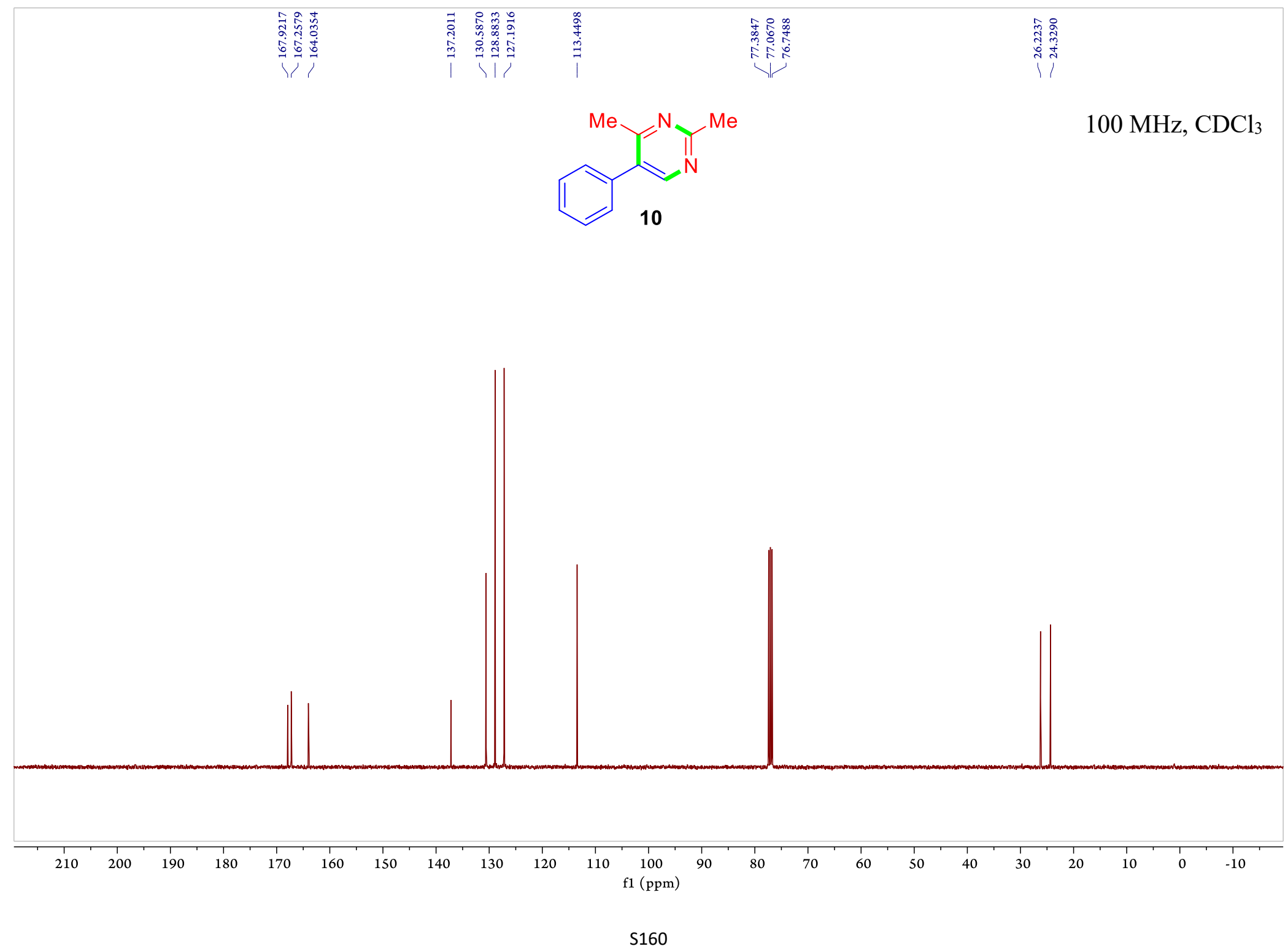




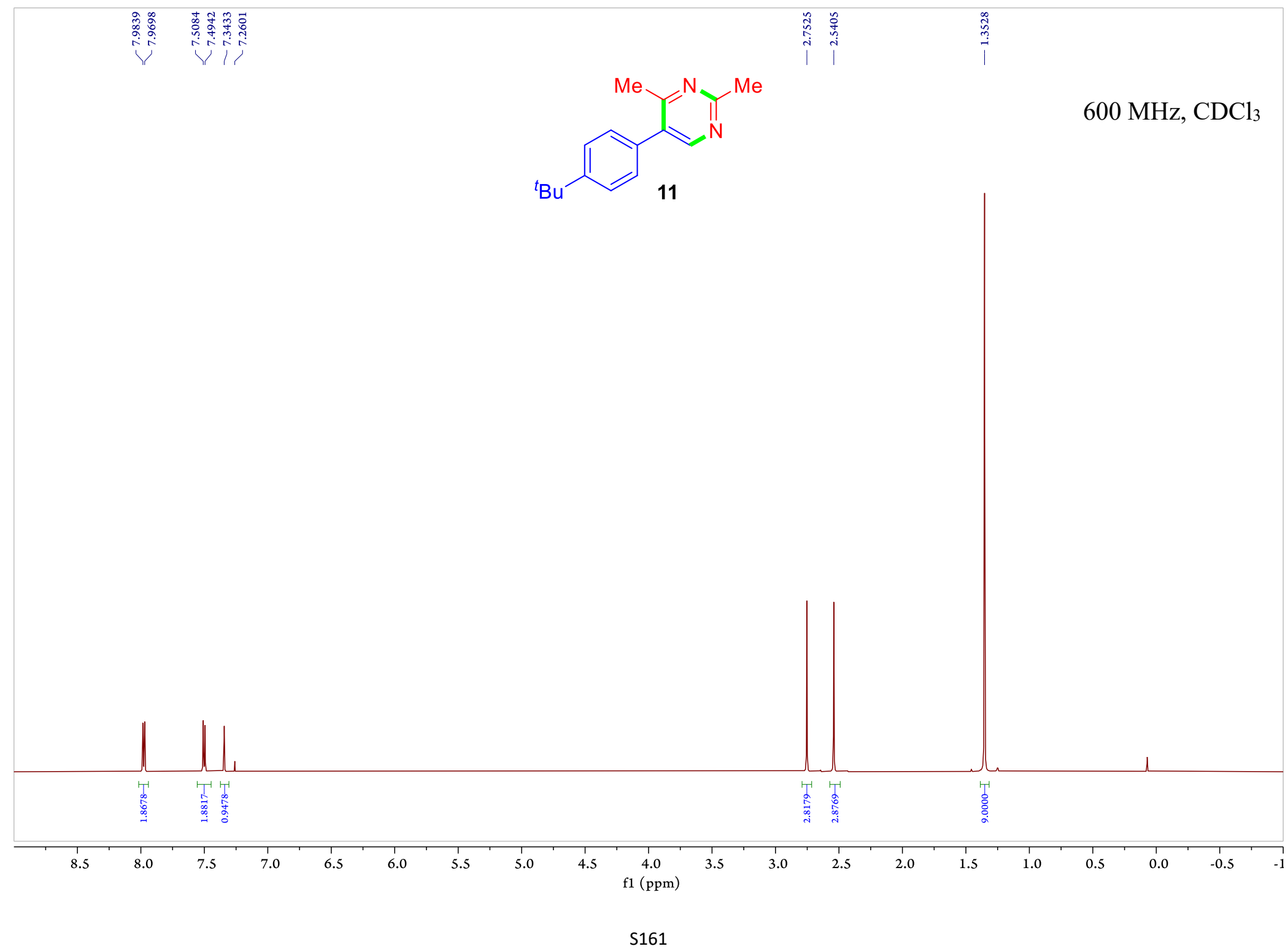




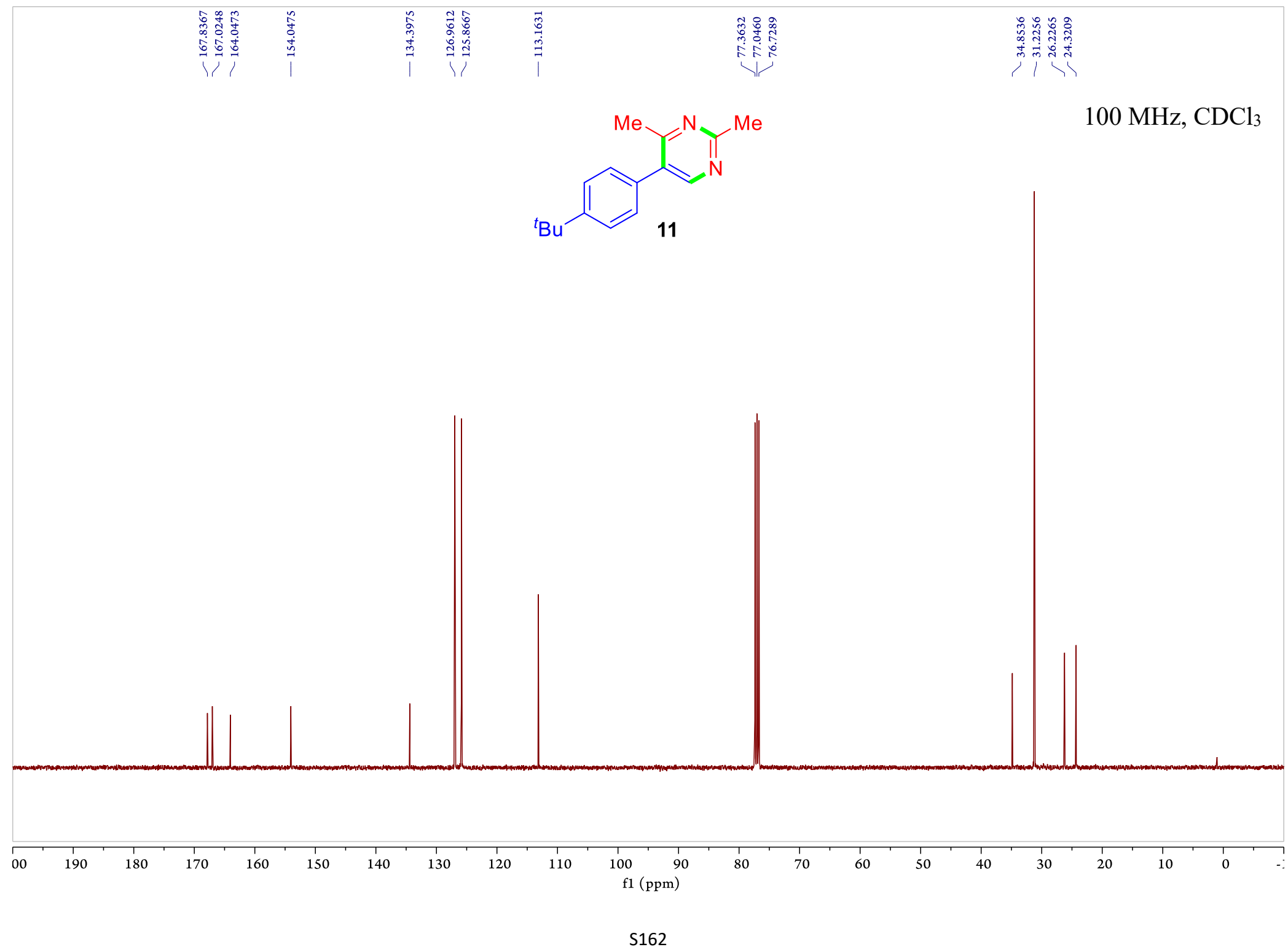



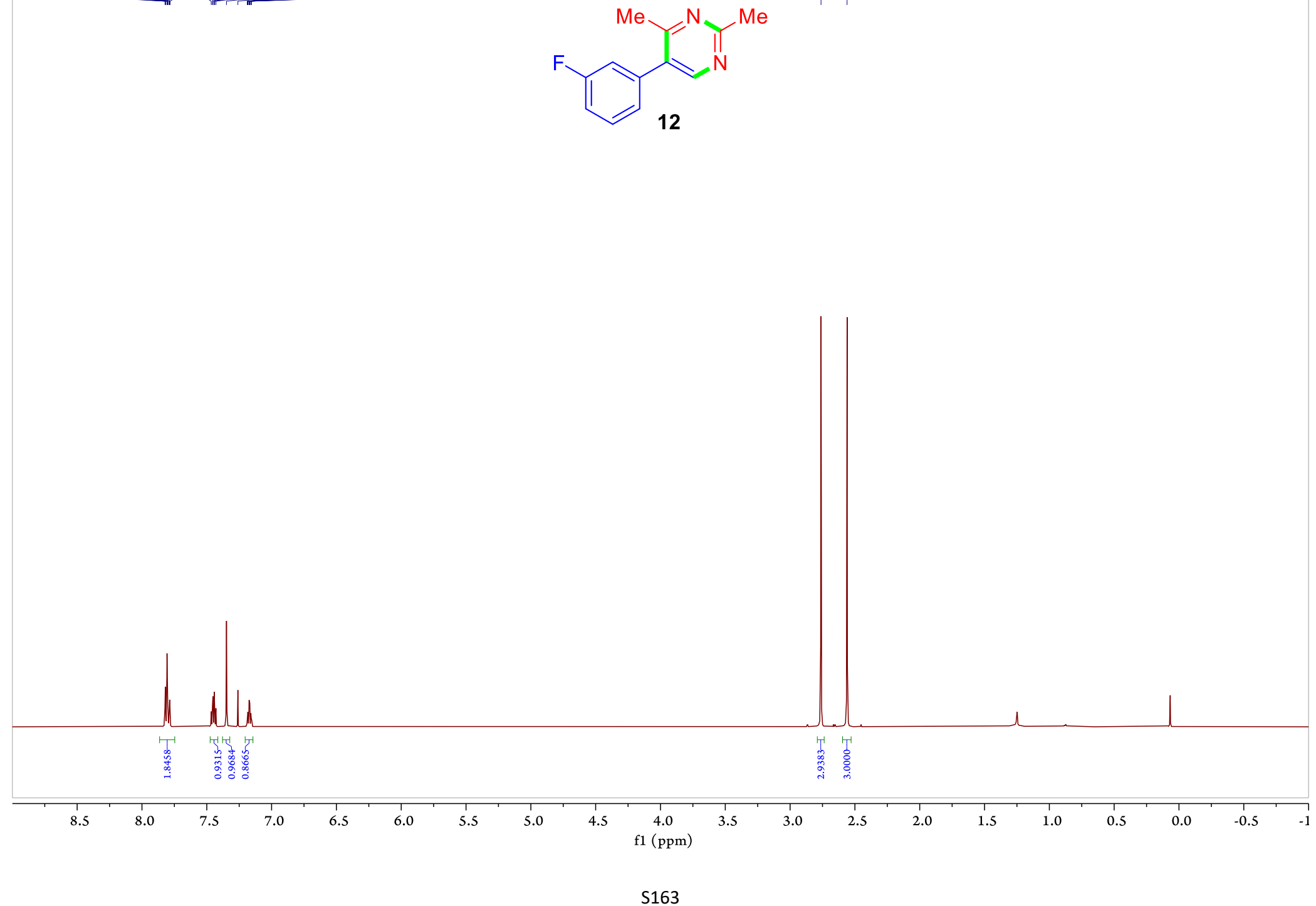


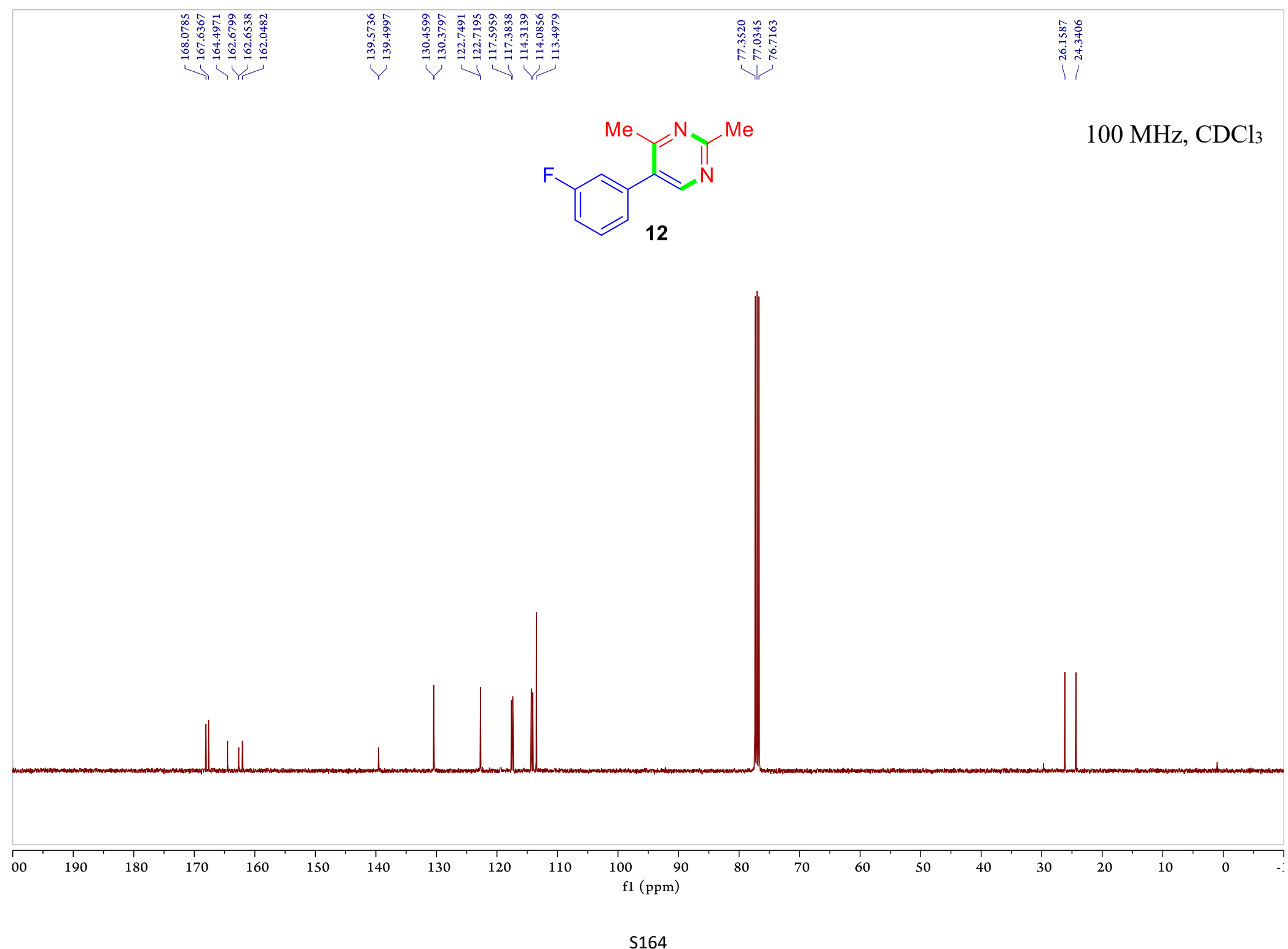




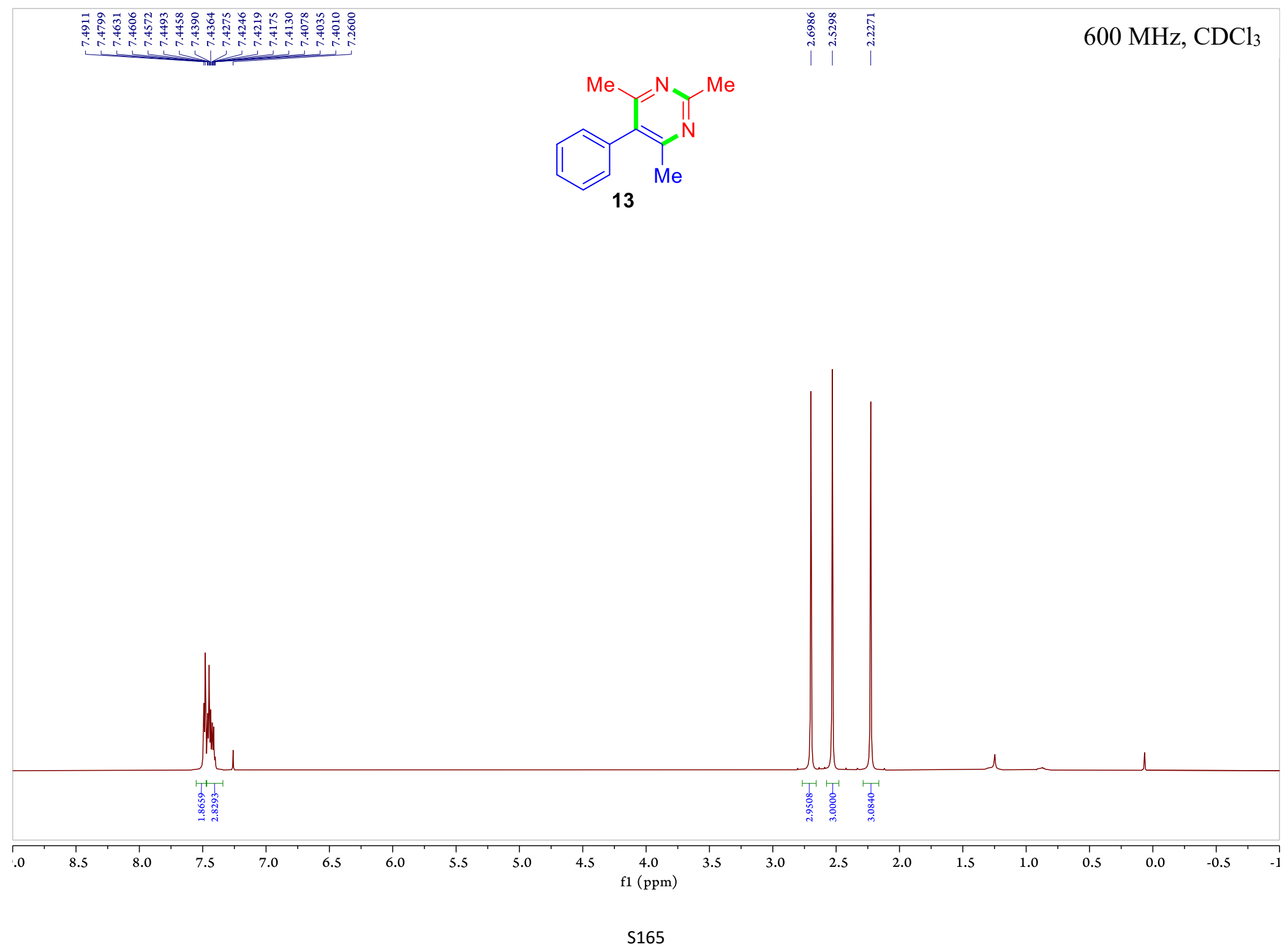



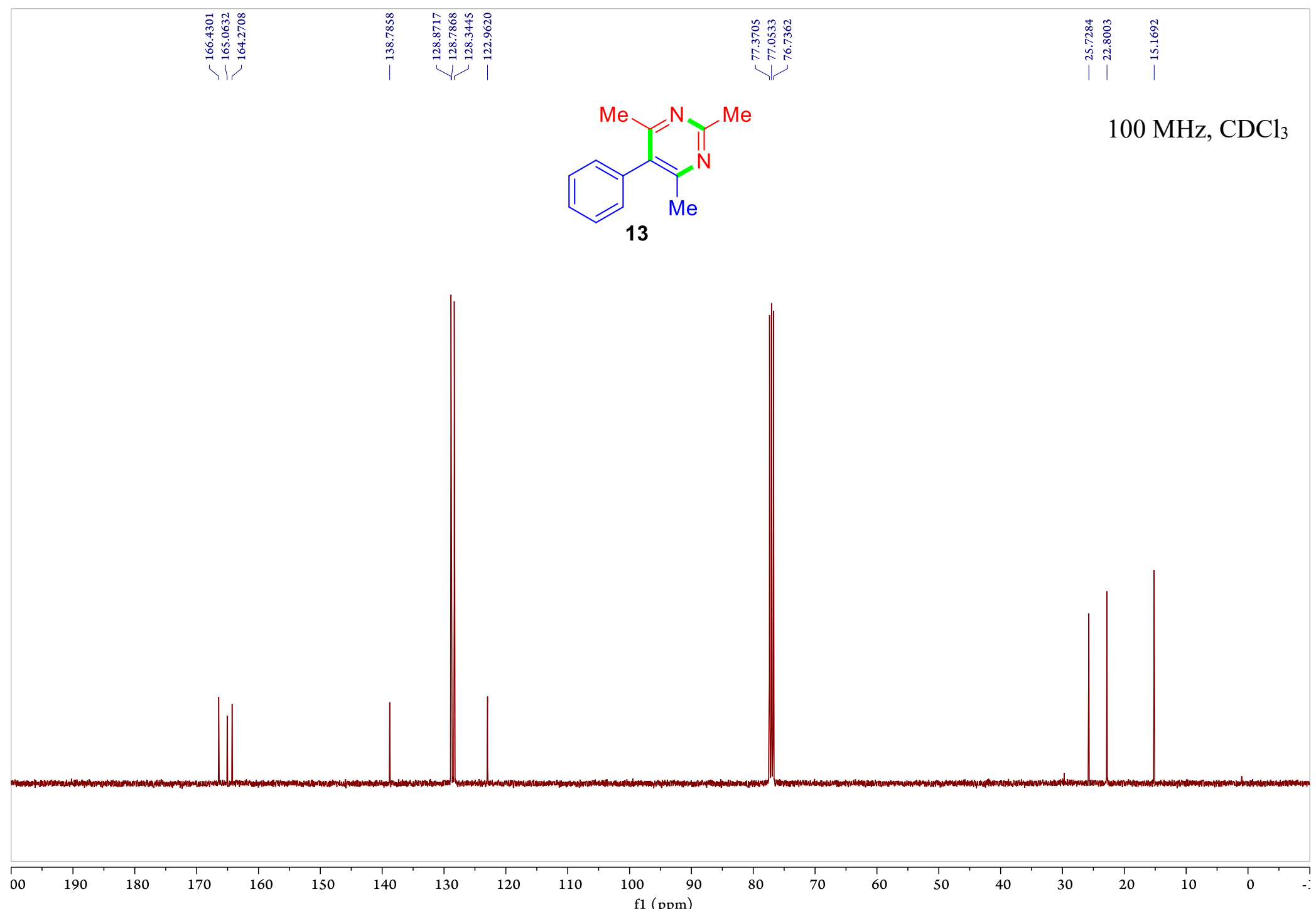


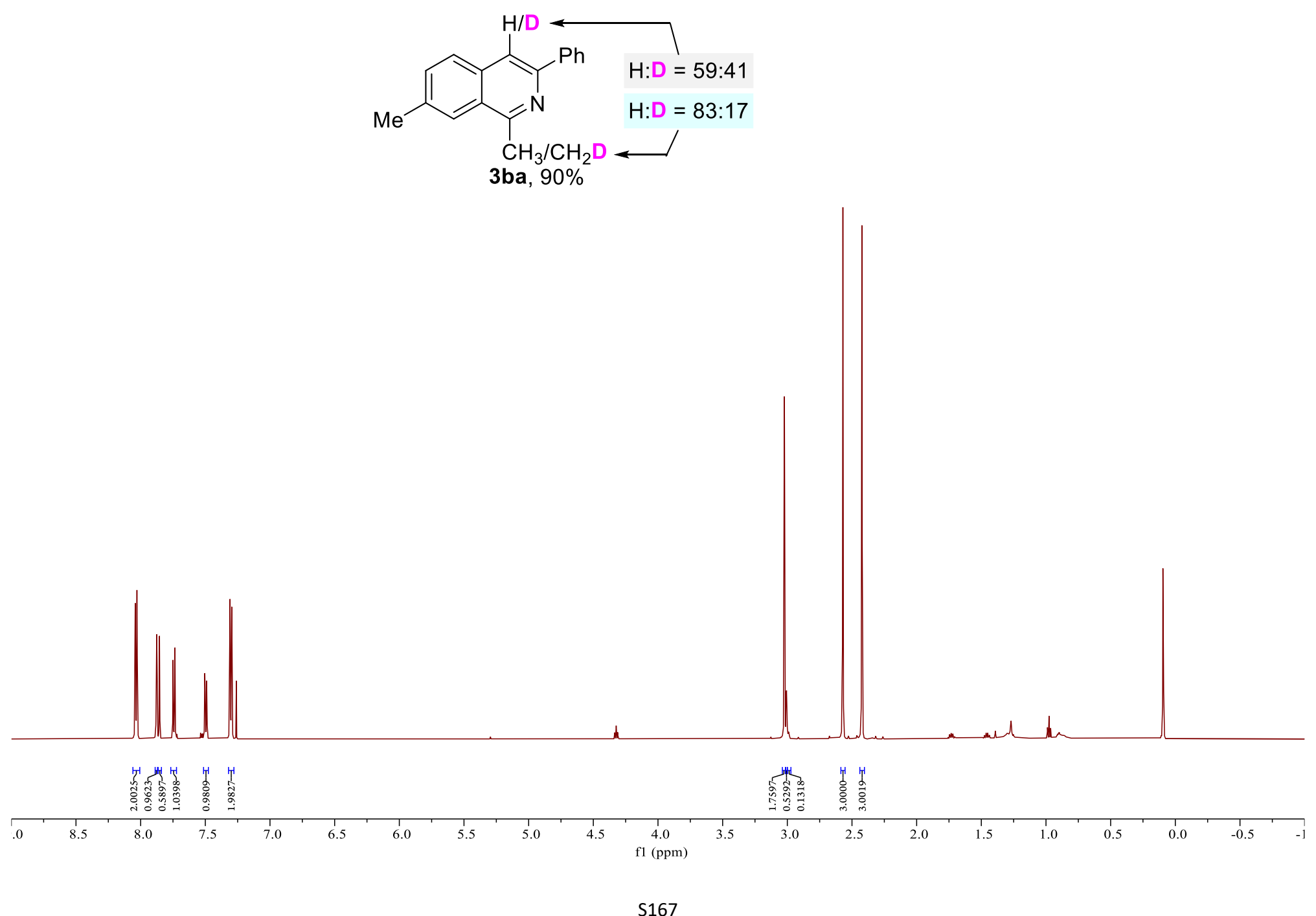



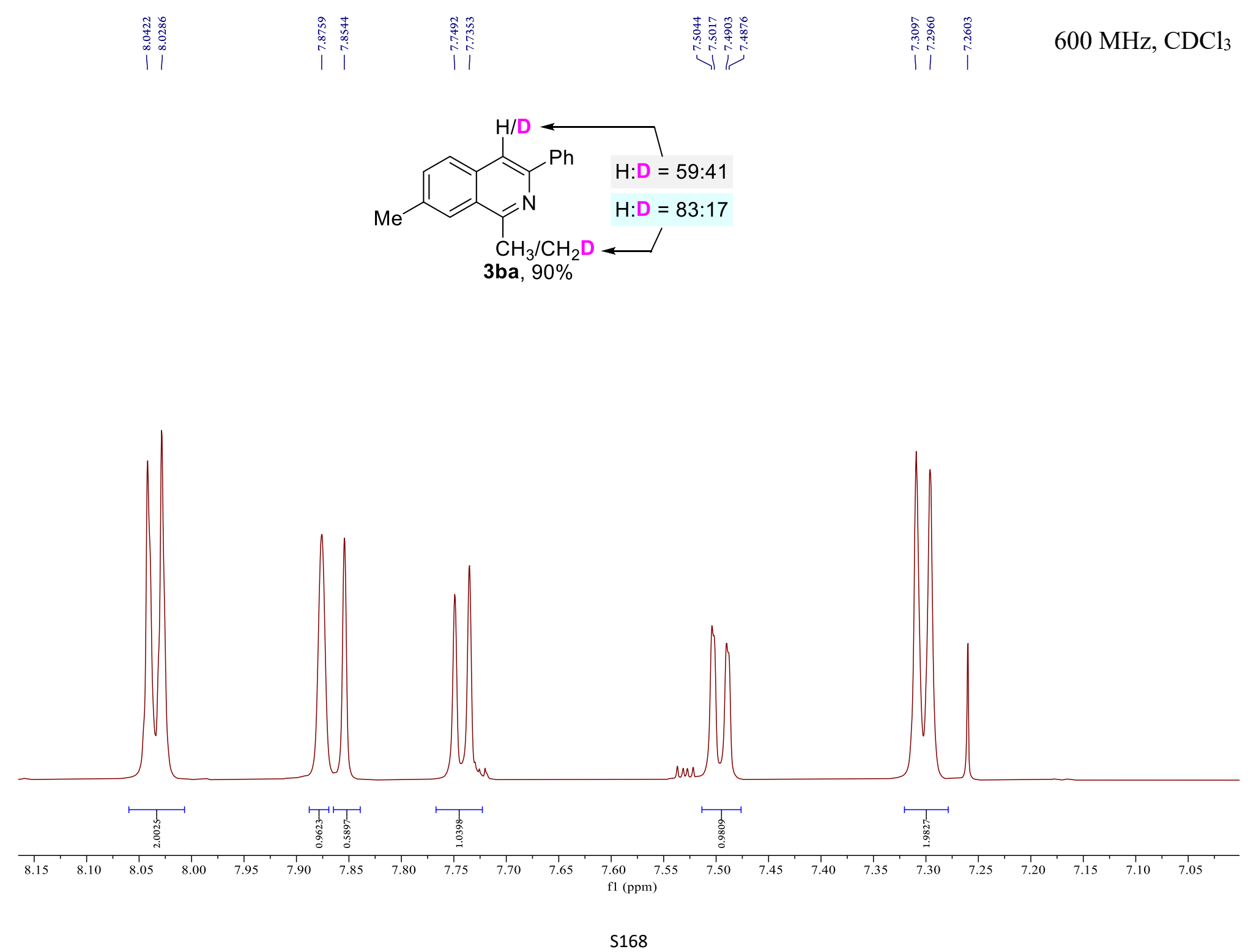


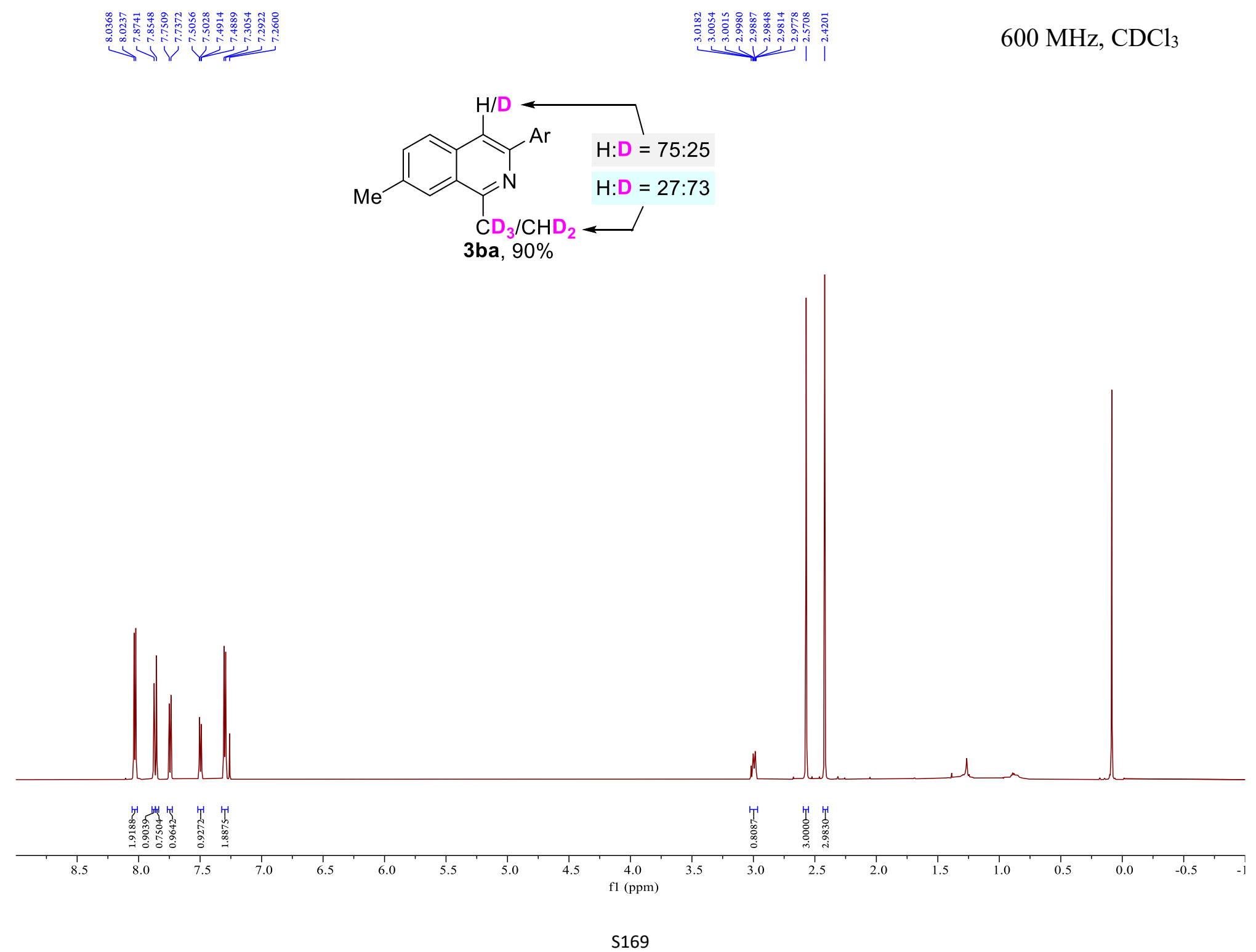



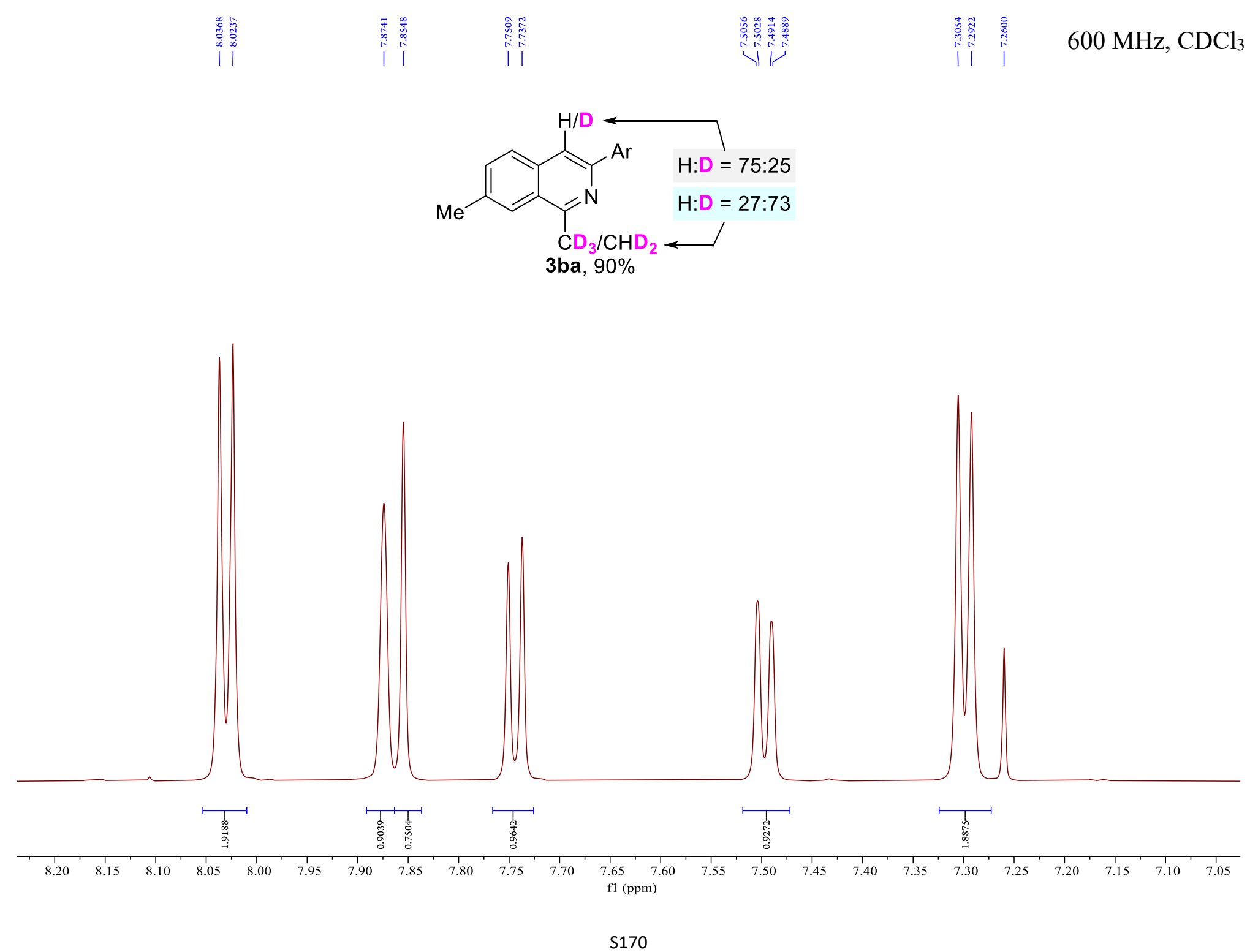
19. Copies of the spectrums for compounds 14 (analogue of intermediate VI)

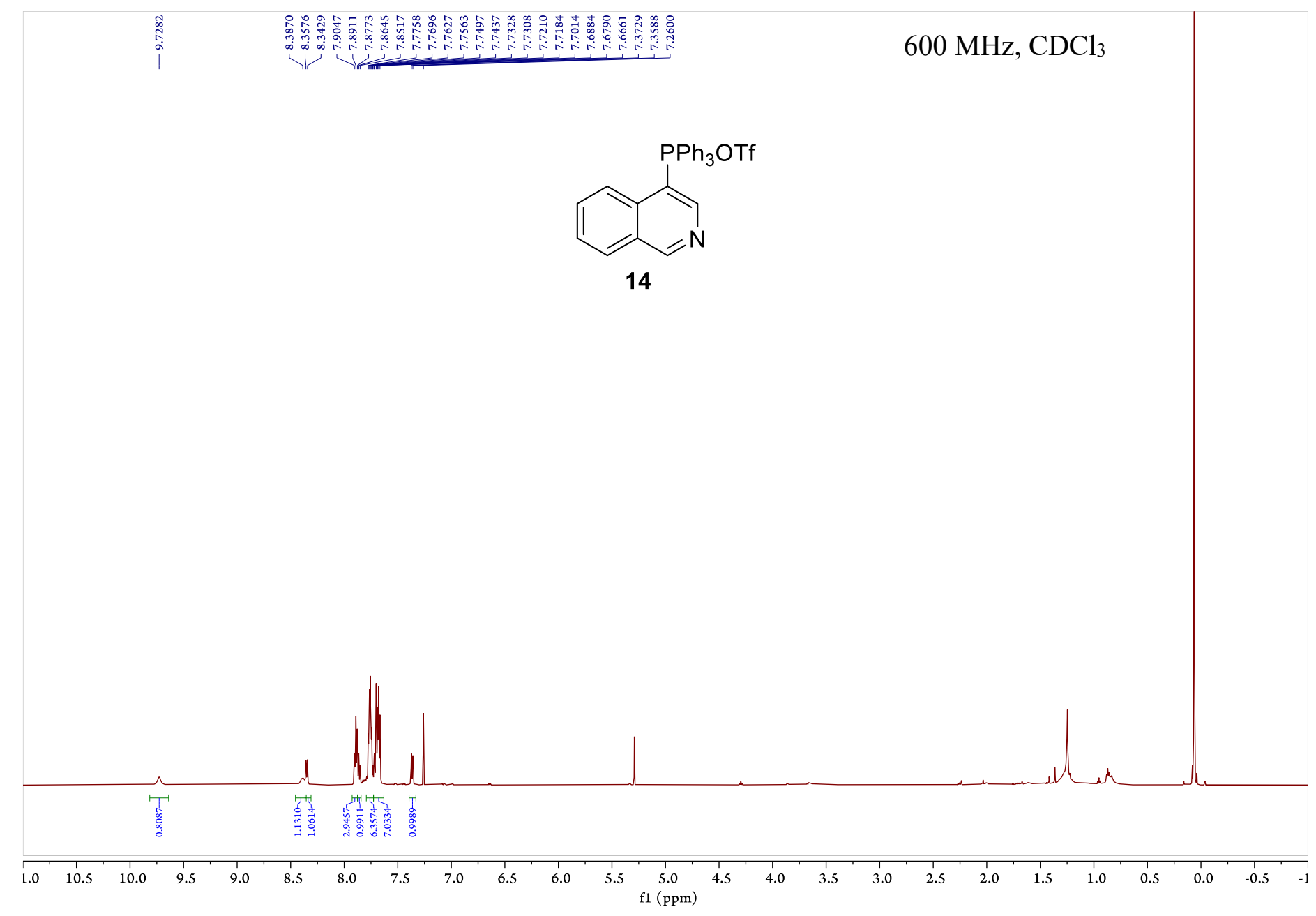




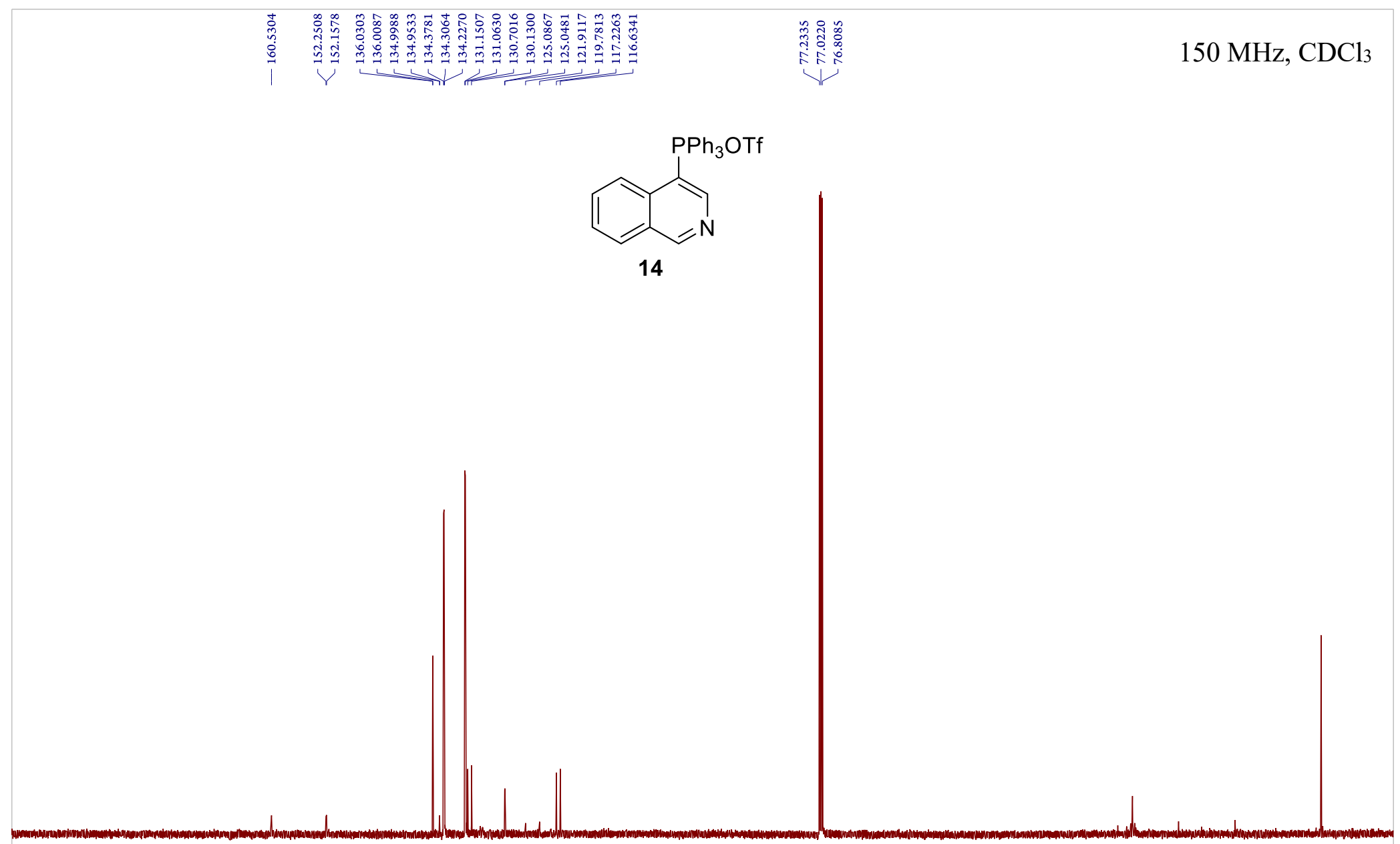

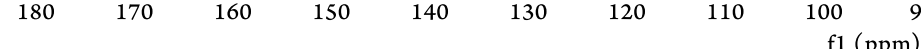




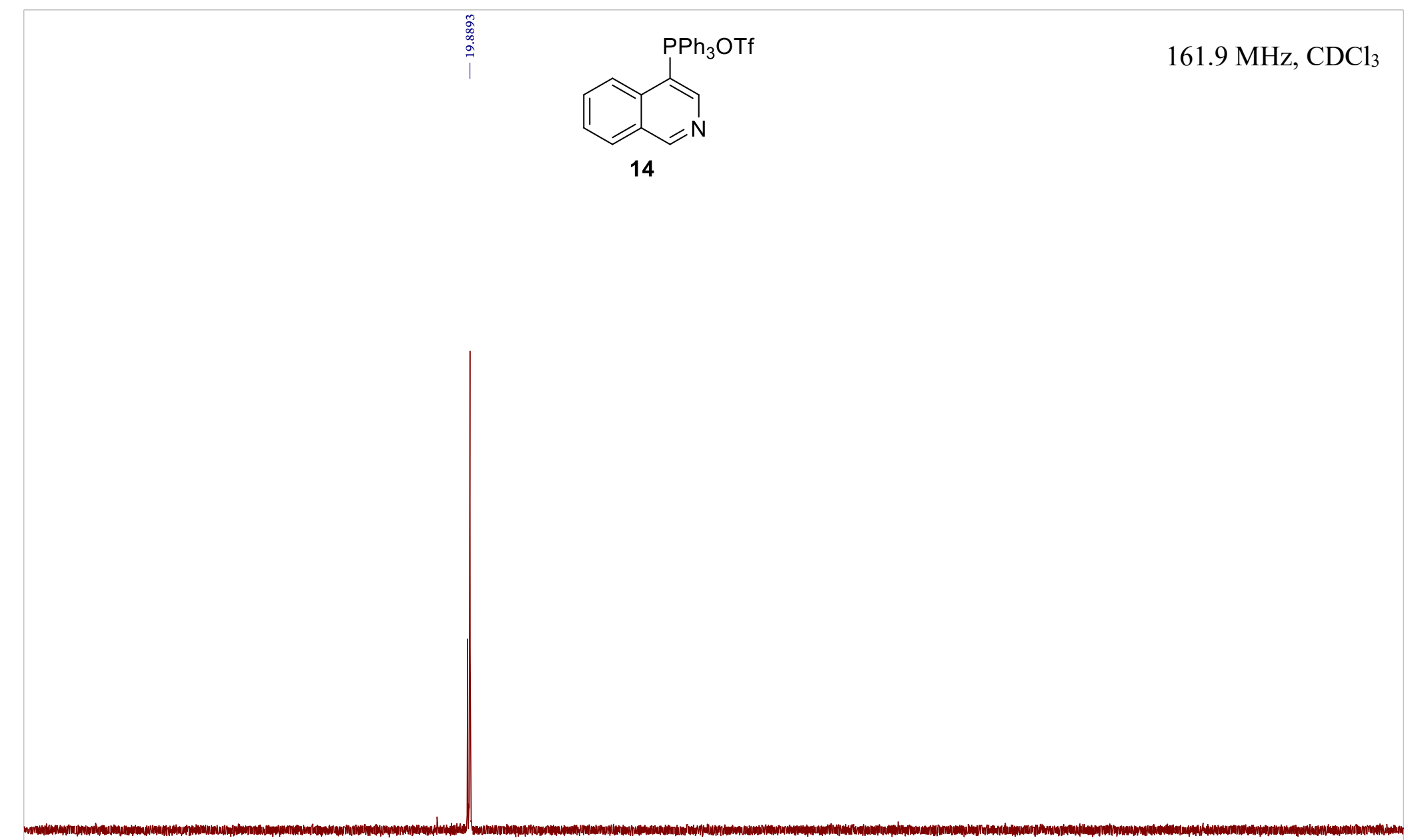

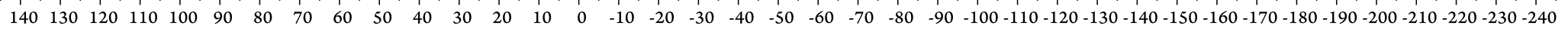
$\mathrm{f} 1(\mathrm{ppm})$ 